\title{
The Economics of Traceability in the New Zealand Dairy Industry
}

\author{
Melissa Welsh
}

\section{VICTORIA UNIVERSITY OF WELLINGTON}

Te Whare Wananga o te Upoko o te Ika a Maui

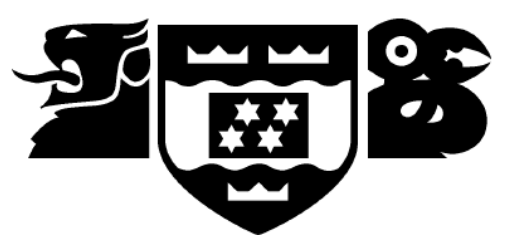

School of Economics and Finance

Te Kura Ohaoha Putea

A Thesis

submitted to the Victoria University of Wellington in fulfilment of the requirements for the degree of

Doctor of Philosophy

in Economics.

Victoria University of Wellington

March 29, 2018 



\begin{abstract}
Dairy is an important dietary component, particularly for young children. Because of this the dairy industry is especially sensitive to contamination scares. Dairy is of particular importance to the New Zealand economy, comprising a significant portion of the country's GDP.

This thesis develops a Markov chain model for the flow of value through the early stages of the dairy supply chain. Using the case of a major New Zealand dairy company, simulations are run under various product testing scenarios. Results point to the importance of where and when testing and interventions take place. Being strict about removing potentially contaminated product early on in the supply chain can reduce total losses, improving overall production output as a result.

Traceability has become an increasingly important research area in recent years. The initial Markov chain model is extended to incorporate parameters for product tracing. By analysing the results of simulations under various scenarios we are able to estimate the value traceability can contribute in the dairy production chain.

Using an event analysis technique with a multi-factor model, the third part of this thesis examines how share prices, in a major New Zealand dairy company, are impacted by shocks. Product recalls, adverse weather events, and demand shocks are considered. Results suggest that individual event characteristics are important, particularly for global demand shocks. Adverse weather events in general are associated with an increase in share price returns. A product recall is associated with the greatest drop in share price returns, emphasising the importance of managing recalls well.
\end{abstract}



For my Grandfather, Barry Edward Richter. Your faith, love and courage will always be an inspiration to me. 



\section{Acknowledgements}

Firstly I would like to thank, my supervisor, Ilan Noy for his support, guidance and wisdom throughout the composition of this thesis. Thank you also to my secondary supervisor Sarah Marshall, her input has been invaluable and her positivity and encouragement in every discussion and correspondence has been greatly appreciated.

I would like to acknowledge GS1 New Zealand for their provision of the scholarship that enabled me to undertake this research.

Thank you to Robert Dabb and Bruce Welsh for your assistance in proofreading various parts of this thesis. Also to my mother, May Dabb, for her much needed regular interruptions and encouragement, throughout my study and particularly in this last year.

I have also benefitted greatly from the support of my fellow PhD Students; Cuong Nguyen, Aditya Kusuma, Diana de Alwis, Rio Yonson, Olivia Wills, Farnaz Pourzand, Jacob Pástor, Belinda Storey, Hanna Habibi, Miles Parker and Azreen Karim.

Finally I would like to thank my husband Michael for his constant support, encouragement and understanding. 


\section{Contents}

1 Introduction $\quad 1$

2 Modelling NZ Dairy Production $\quad 9$

2.1 Introduction . . . . . . . . . . . . . . . . . 9

2.2 Dairy Production and Supply Chain Modelling . . . . . . . . . 10

2.3 Dairy in New Zealand . . . . . . . . . . . . . . . . 13

2.3.1 Processing In the Factory . . . . . . . . . . . 14

2.3.2 Receiving Milk at the Factory . . . . . . . . . . . 15

2.3.3 The Milk Tanker . . . . . . . . . . . . . . . . 15

2.3.4 On the Farm . . . . . . . . . . . . . . 15

2.3.5 Testing . . . . . . . . . . . . . 17

2.4 Developing the Model . . . . . . . . . . . . . . . . 17

2.4.1 The Deterministic Model . . . . . . . . . . . . . 18

2.4.2 Product Flow in a Discrete Time Markov Chain Model 24

2.5 The Single Event Model . . . . . . . . . . . . . . . . 26

2.5.1 Parameter Values . . . . . . . . . . . . . . . . 29

2.5.2 Single-Event Model Simulations . . . . . . . . . . . 32

2.6 Developing The Multi-Event Model . . . . . . . . . . . . 36

2.6.1 The Milk Tanker . . . . . . . . . . . . . . . 37

2.6.2 The Factory Reception . . . . . . . . . . . . . . . 38

2.6.3 The Processing Stage . . . . . . . . . . . . . . 41

2.6.4 Summary equations and Stage Interactions . . . . . . . 43

2.7 Multi-event Model Simulations . . . . . . . . . . . . . . . . . . 48

2.7.1 Parameter Values . . . . . . . . . . . . . . . . . 49

2.7.2 The Best of all Possible Worlds Scenario . . . . . . . . 51 
2.7.3 Testing Scenarios . . . . . . . . . . . 53

2.7.4 Discussion . . . . . . . . . . . . . . 61

2.8 Conclusions . . . . . . . . . . . . . . . . 63

3 Traceability From Farm to Factory 65

3.1 Introduction . . . . . . . . . . . . . . . . 65

3.2 Dairy . . . . . . . . . . . . . . . . 67

3.3 Traceability . . . . . . . . . . . . . . . . . . 68

3.4 Markov Chain Model . . . . . . . . . . . . . . . . . 72

3.4.1 Problem Statement . . . . . . . . . . . . . . . 72

3.4.2 Product Flow DTMC Model . . . . . . . . . . . . . 72

3.4.3 Developing the Model with Traceability . . . . . . . . 75

3.4.4 The Milk Tankers . . . . . . . . . . . . . 76

3.4.5 The Factory Reception Stage . . . . . . . . . . . . . . 81

3.4.6 The Processing Stage . . . . . . . . . . . . . 86

3.4.7 Summary equations and Stage Interactions: . . . . . . 92

3.5 Model Simulation Results and Discussion . . . . . . . . . . . . 100

3.5.1 Parameter Values for Dairy in New Zealand . . . . . . 101

3.5.2 Reducing Factory Reception Rejection . . . . . . . . . 108

3.5.3 Reducing Product Loss at Processing Entry . . . . . . 112

3.5.4 Reducing Product Loss Throughout the Supply Chain 114

3.6 Conclusion . . . . . . . . . . . . . . . . . . . . 117

4 Recalls, Storms and Import Bans $\quad 119$

4.1 Introduction . . . . . . . . . . . . . . . . . . . . . . 119

4.1.1 Assessing the Impact of Shocks on Share Prices . . . . 120

4.1.2 The New Zealand Dairy Industry . . . . . . . . . . . . 122

4.2 Events . . . . . . . . . . . . . . . . . . 125

4.2.1 Product Recalls . . . . . . . . . . . . . . 125

4.2.2 Adverse Weather Events . . . . . . . . . . . . . 128

4.2.3 Global Supply and Demand Shocks . . . . . . . . 130

4.3 Methodology and Data . . . . . . . . . . . . . 131

4.3 .1 Data . . . . . . . . . . . . . . . 132 
4.3.2 Model Construction . . . . . . . . . . . . . . . 138

4.3.3 Event Study Methodology . . . . . . . . . . . . . . 140

4.4 Analysis and Results . . . . . . . . . . . . . . . . . . . . . . 141

4.4.1 Overarching model . . . . . . . . . . . . . . . . 141

4.4.2 Product Contamination and Recalls . . . . . . . . . . . 143

4.4.3 Adverse Weather Events . . . . . . . . . . . . . . . . 151

4.4.4 Russian Ban of European Union Farm imports . . . . . 158

4.4.5 End of European Union Milk Quotas . . . . . . . . . . 162

4.5 Discussion and Conclusions . . . . . . . . . . . . . . . 165

$\begin{array}{ll}\text { Appendices } & 168\end{array}$

4.A GDT Price Index . . . . . . . . . . . . . . . . . . 168

4.B Complete Overarching Model . . . . . . . . . . . . . . . . 168

5 Summary and Conclusions 171

$\begin{array}{lr}\text { Bibliography } & 175\end{array}$ 


\section{List of Figures}

1.1 The number of recall and withdrawal event notifications received by ProductRecallNZ each quarter from 2012 to 2017 . . . . . . . . 3

1.2 The number of products recalled or withdrawn through ProductRecallNZ each quarter from 2013 to 2017. . . . . . . . . . 4

1.3 The number of businesses involved in recalls or withdrawals notified to ProductRecallNZ. . . . . . . . . . . . . . . 4

1.4 The number of businesses involved in recalls or withdrawals notified to ProductRecallNZ, per 1000 enterprises. . . . . . . . . 5

2.1 The overall picture of the dairy production chain, from the farm to initial processing at the factory. . . . . . . . . . 14

2.2 The flow of value into and out of the tanker stage. $T(t)$ is the value in the tanker stage at time $t \ldots \ldots \ldots$. . . . . . . . . 19

2.3 The movement of product into and out of the factory reception stage. 21

2.4 The rate of value flow into and out of the processing stage. . . . . 22

2.5 Flow chart showing the path milk takes from the farm to the factory and the decisions that are made along the way. . . . . . . 25

2.6 The amount of milk output after the three stages of processing in our model. Both a stochastic simulation and the deterministic model solution are shown over a ten hour period. . . . . . . . . 33

2.7 The amount of milk contained in each stage over a ten hour simulation. . . . . . . . . . . . . . . . 34

2.8 An hour from the middle of Figure 2.7 focusing on the simulation of the Milk Tanker and Factory Reception stages. . . . . . . . . 35

2.9 Probability tree showing the possible outcomes when milk is delivered to the factory. The rejection of a tanker compartment is denoted by $\mathbf{r}$, while a denotes acceptance. . . . . . . . . . . . 39 
2.10 A simulation and the deterministic solution for the total value of the milk produced over a 24 hour period. The transition probabilities used are summarised in Section 2.6.4, and the parameter values are summarised in Table $2.5 . \quad$. . . . . . . . . . . . 52

2.11 Plots of a simulation and the deterministic solution for the value of milk contained in each stage of the supply chain over 24 hours. The transition probabilities used are summarised in Section 2.6.4, and the parameter values are summarised in Table 2.5. . . . . 53

2.12 The total value of the milk produced over a 24 hour simulation period, along with the deterministic solution. The transition probabilities are those described earlier and summarised in Section 2.6.4. The parameters' values are summarised in Table 2.7. . . . . . . 56

2.13 The value of milk in each stage, over 24 hours of simulation, and the deterministic solution. The parameters' values are summarised in Table 2.7. . . . . . . . . . . . . . . . . . . . 57

2.14 The total value of the milk produced over a 24 hour period for seven different vat acceptance probabilities. All other parameters remain constant at the values given in Table $2.7 \ldots$. . . . . 58

2.15 The total value of the milk produced over a 24 hour period for seven different milk factory reception acceptance probabilities. All other parameters remain constant at the values in Table 2.7. . . . 59

2.16 The total value of the milk produced over a 24 hour period for seven different milk tanker acceptance probabilities. The value of $\gamma$ is varied between 0.75 and 1 , while $\alpha$ and $\beta$ remain the same as in Table 2.7, along with the rest of the parameters. The step size is $\Delta t=8$ Seconds. . . . . . . . . . . . . . . . .

3.1 Flow chart showing the path milk takes from the farm to the factory and the decisions that are made along the way. . . . . . . . 74

3.2 The path of milk from the farm to the factory. Each time the milk enters a new stage testing and potential rejection is depicted by vertical dashed lines. . . . . . . . . . . . . . . . . . . 75

3.3 The flow of value into and out of the tanker stage. $T(t)$ is the value in the tanker stage at time $t . \ldots \ldots . . \ldots 78$

3.4 The rate of value flow into and out of the factory reception stage. . 81

3.5 Probability tree showing the possible outcomes when milk is delivered to the factory. . . . . . . . . . . . 83

3.6 The rate of value flow into and out of the processing stage. . . . . 87

3.7 Probability tree for the outcomes when passing product from the factory reception stage to processing. . . . . . . . 88 
3.8 Simulations of milk produced over a 24 hour period with a $25 \%$ rejection rate of product entering the factory reception. One simulation of each scenario is shown. . . . . . . . . . . . . . 109

3.9 Box plots for the value of milk processed over a 24 hour period in various scenarios, with and without traceability, over five hunderd simulations. The confidence interval of the mean is also shown as a small green box within each plot. . . . . . . . . . . . . . . 111

3.10 A single simulation of milk produced over a 24 hour period, with a $25 \%$ rejection rate of product entering the processing stage. . . . 113

3.11 Box plots for five hundred simulations of each scenario given in Table 3.4. The $95 \%$ confidence interval for the mean is also shown as a green small box within each plot. . . . . . . . . . . . 115

3.12 A simulation of milk produced over a 24 hour period with a $25 \%$ rejection rate of product entering the factory reception and processing stages. . . . . . . . . . . . . . . . 116

3.13 Box plots produced after five hundred simulations for the rejection scenarios given in Table 3.5. Both traceability and non-traceability scenarios are shown. The small green box within each plot shows the $95 \%$ confidence interval for the mean. . . . . . . . . . . . . 117

4.1 Actual FCG returns plotted along with the normal returns as estimated by the fifty day individual event model and the overarching model. The vertical black line marks 22 May 2013, the day the Mainland cheese recall was announced. . . . . . . . . . . . . . . 146

4.2 Actual FCG returns plotted along with the predicted normal returns using the seventy five day individual event model and the overarching model. The vertical black line marks 3 August 2013, the day the WPC80 contamination incident was announced. . . . . 147

4.3 Actual FCG returns plotted along with the normal returns as predicted by the seventy five day individual event model from Table 4.6. The vertical black line marks 13 January 2014, the day the cream recall was announced. . . . . . . . . . . . . . . . . 148

4.4 Actual FCG returns plotted along with the returns predicted by seventy five day event model. The first vertical black line marks 10 September 2013, the day the storm hit. The second line marks 12 September 2013, the end of the storm. . . . . . . . . . . . . 154

4.5 Actual FCG returns plotted along with the seventy five day normal returns model. The first vertical black line marks 8 July 2014, the first day of the storm. The second line marks 11 July 2014, the end of the storm . . . . . . . . . . . . . . . 155 
4.6 Actual FCG returns plotted along with the normal returns model. The vertical black line marks 21 March 2016, the day of the storm. 157

4.7 Actual FCG returns plotted along with the normal returns model. The vertical black line marks 7 August 2014, the day the import ban came into effect. . . . . . . . . . . . . . . . 160

4.8 Actual FCG returns plotted along with the returns predicted by each model. The vertical black line marks 31 March 2015, the date the quota system ended. . . . . . . . . . . . . . . 164 


\section{Chapter 1}

\section{Introduction}

In order to produce quality products that are safe for consumers, firms need to have robust testing and quality assurance systems in place. In some situations a fault may temporarily slip through this system or there is a necessary delay in obtaining test results that continued production cannot wait for. We need a traceability system to keep track of where affected product may be at any point in time.

Traceability is the capability to trace goods throughout the production and distribution chain (Tamayo et al., 2009). The aim of a traceability system is to collect information relevant to the location and interaction of products as they travel through the supply chain (Dabbene \& Gay, 2011; Karlsen et al., 2013), allowing the flow of material to be followed. This has become an increasingly important research area in recent years. Increasing complexity in widespread international supply chains, along with varying standards across countries, means traceability has become even more important in maintaining reputations and trade. It has always been an important aspect of production, but recent contamination events have highlighted its significance.

In New Zealand the 2013 botulism scare in particular highlights the need for fast, accurate testing and traceability (WPC 2013 Response Team). The amount of time taken in the scare of 2013 to confirm the sources of the contamination and the location of the contaminated batches, risked not only Fonterra's reputation but New Zealand's reputation as an exporter as well. Traceability makes selective recalls possible (Tamayo et al., 2009), and is 
crucial to a firm's ability to limit the size and spread of a recall (Buhr, 2003). How well a traceability system is implemented will influence how effective it is in a recall situation. The precision of the system will determine how much product needs to be recalled, affecting the total value lost and the costs associated with locating and removing affected product (Dabbene \& Gay, 2011). The ability to trace both forwards and backwards through the distribution chain is key. A firm must be able to trace back to the source of the contamination, then forwards to identify all of the affected product (Jansen-Vullers et al., 2003). With poor traceability it is difficult to determine how far a contamination has gone, necessitating a widespread recall and the very real possibility of contaminated product being consumed. An effective traceability system allows the efficient and precise withdrawal of contaminated product from the distribution chain, mitigating the costs associated with a contamination, and reducing potential risk to consumers health (Resende-Filho \& Buhr, 2010).

Traceability is a widely used interdisciplinary concept, with various approaches studied in fields spanning the natural and social sciences over the last few decades (Karlsen et al., 2013). A good traceability system will also reduce anonymity throughout the distribution chain, making it easy to identify who may be liable in the event of a contamination or fault (Resende-Filho \& Buhr, 2010). This can provide further incentive for all parties contributing to a supply chain to ensure they are providing safe goods. The more accountability a firm is held to, the more they seek to improve their own standards (Carriquiry \& Babcock, 2007; Pouliot \& Sumner, 2008).

Product recalls occur when a product is found to be unsafe or unsuitable for consumers. They can be divided into two main types: consumer level recalls and trade level recalls. A consumer level recall involves the removal of product from the distribution chain and extends to product sold to consumers. A trade level recall, more commonly referred to as a withdrawal, involves the removal of product from the distribution chain, but does not extend to consumers. Withdrawals do not require public notification at all (MPI, 2017), as a consequence they often do not receive any media attention.

About $90 \%$ of product recalls and withdrawals go through ProductRecallNZ, a service run by GS1 New Zealand. Figure 1.1 shows the number of recall and withdrawal notifications they received each quarter from June 2012 to 
March 2017 (GS1 NZ, 2017).

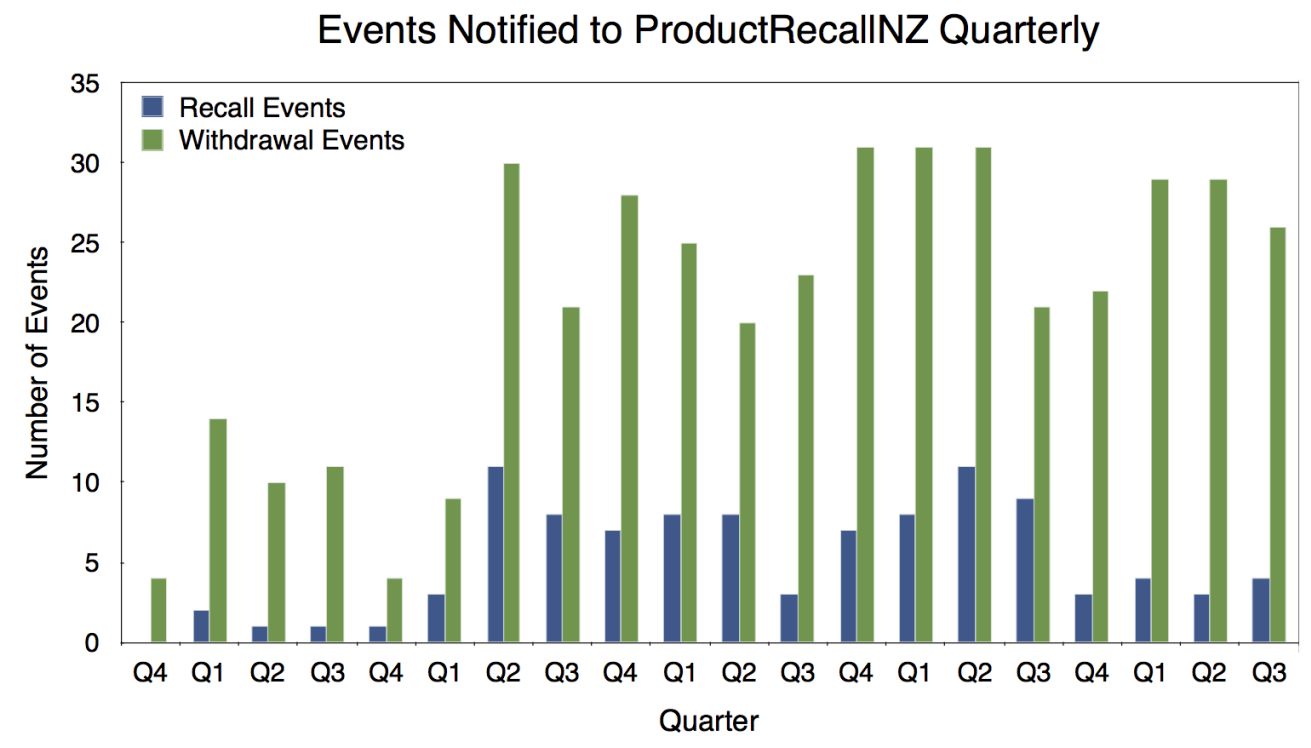

Figure 1.1: The number of recall and withdrawal event notifications received by ProductRecallNZ each quarter from 2012 to 2017.

The number of consumer level recalls ranges from zero to eleven per quarter, while the number of trade level withdrawals ranges from four to thirty one. On average there were five recalls per quarter compared to twenty one withdrawals. Approximately $80 \%$ of these events were trade level withdrawals the public knew nothing about.

We do not have access to the actual value of products involved in each of these recalls, or the industry they belong to, however for each of these events a number of different products are affected. To help get an idea of the potential size of these recall events Figure 1.2 shows the number of different individuals products recalled or withdrawn, through ProductRecallNZ, each quarter from March 2013 to March 2017 (GS1 NZ, 2017).

The number of products recalled each quarter over this period, ranges from 16 to 119 , with an average of 63 . The number of products withdrawn ranges from 58 to 221 per quarter, with an average of 147 . On average about $70 \%$ of products removed from the distribution chain are withdrawn at the trade level.

Comparing Figures 1.1 and 1.2, we see the number of products withdrawn or recalled each quarter is significantly higher than the number of events. The lowest number of products withdrawn, in the first quarter of 2014 was still 
Product Recalls or Withdrawals

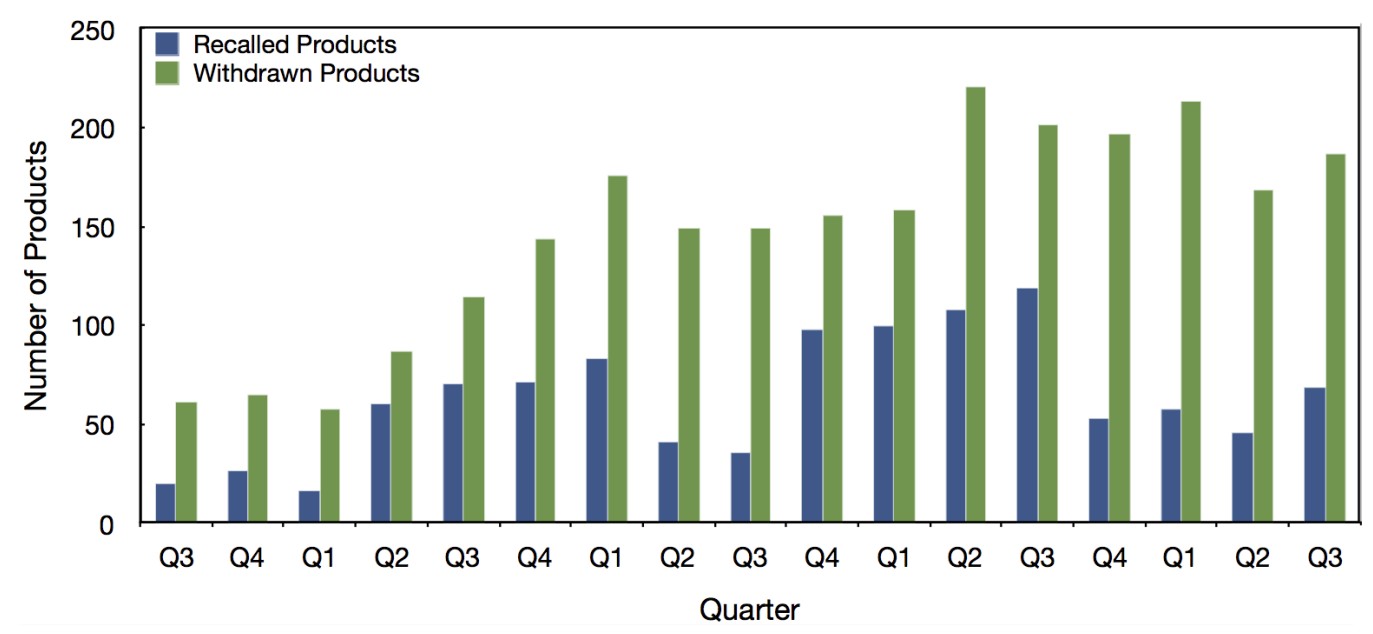

Figure 1.2: The number of products recalled or withdrawn through ProductRecallNZ each quarter from 2013 to 2017.

just over fifty. The quarters with the most withdrawal events barely reach thirty notifications. This suggests that multiple products are often involved in each recall or withdrawal event. Each product recalled or withdrawn can result in thousands of items needing to be removed from the distribution chain. This implies a large amount of substandard items, involving many businesses and brandnames, essentially hidden from public view.

Figure 1.3 shows the number of business involved in recalls or withdrawals managed through ProductRecallNZ from 2013 to 2017. There is a steady

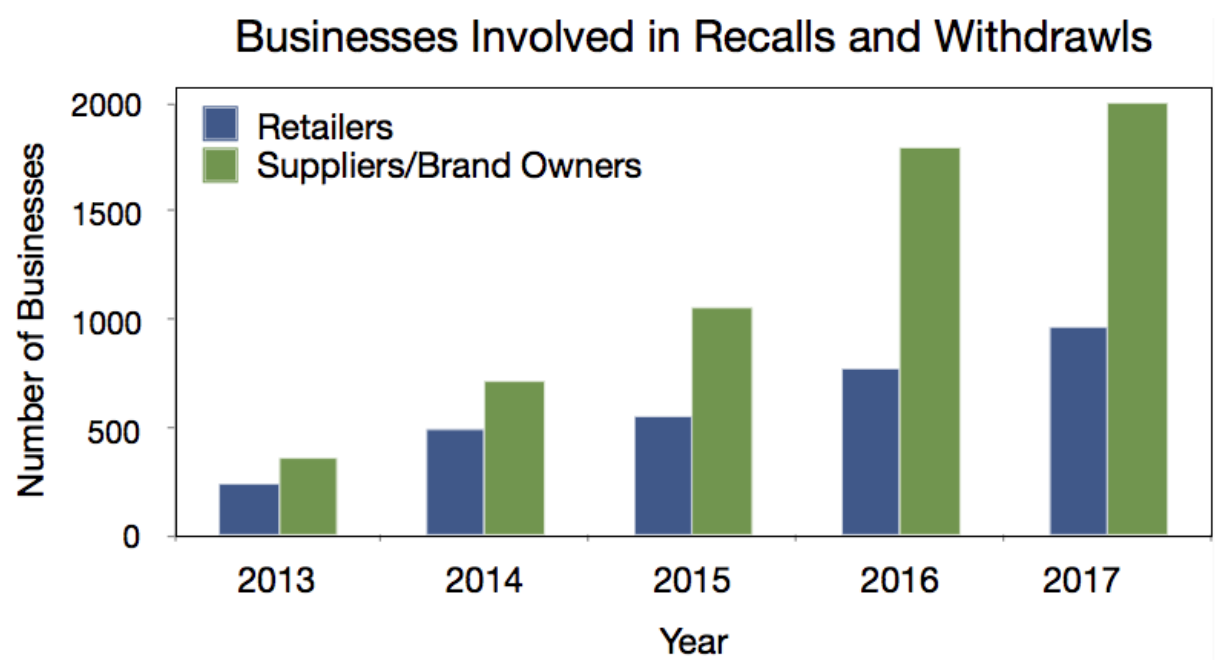

Figure 1.3: The number of businesses involved in recalls or withdrawals notified to ProductRecallNZ. 
increase in the number of businesses involved in recalls and withdrawals over time. Such a consistent increase was not seen in the number of events in Figure 1.1, or in the number of products removed in Figure 1.2. This suggests a growing interconnectedness in New Zealand's distribution chain, increasing the importance of efficient product removal systems. The greater number of suppliers and brand owners involved, compared with retailers, reflects the higher number of withdrawals compared to recalls. Retailers are not always affected by withdrawals, as they sell directly to consumers. If retailers are affected it is more likely a consumer level recall will be necessary. The total number of businesses involved in these recalls, is presented as a proportion of the total number of enterprises in New Zealand in Figure 1.4. This figure shows the number of businesses involved in recalls for every 1000 businesses registered in New Zealand at February for each year from 2013 to 2017, as recorded by StatsNZ (2017a). We see a similar pattern of increase in this

\section{Businesses Involved in Recalls and Withdrawals}

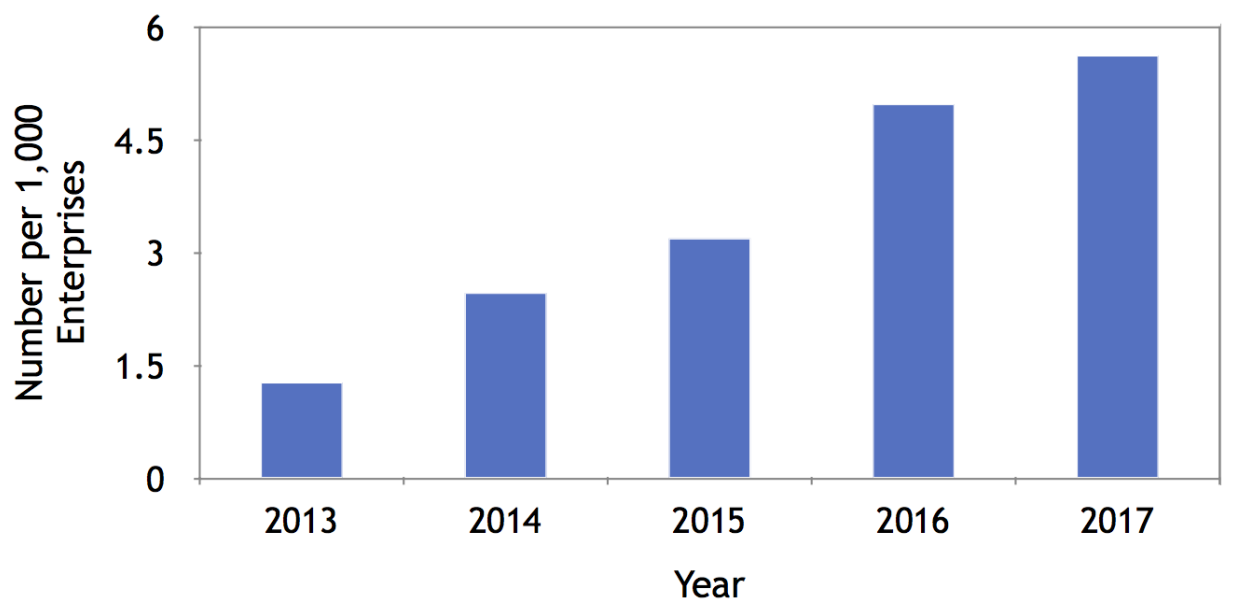

Figure 1.4: The number of businesses involved in recalls or withdrawals notified to ProductRecallNZ, per 1000 enterprises.

figure to what we saw in Figure 1.3. This suggests that the growing number of business involved in recalls is not just due to an increased number of businesses operating.

Some firms may be hesitant to adopt a new traceability system because of its costs. It can be difficult to estimate the benefits of a traceability system (Dupuy et al., 2005). The main value of a traceability system lies in its ability to improve the recall process though records management and verification 
(Resende-Filho \& Buhr, 2010). The benefits of having such a system are generally only seen in the losses, both direct and indirect, that it prevents in the event of a recall (Dupuy et al., 2005). This thesis aims to investigate the value of traceability in the context of the New Zealand dairy industry.

Following the WPC80 botulism scare, traceability standards in the New Zealand dairy industry have come under close scrutiny. Current standards for traceability in New Zealand vary across industries. Food safety standards do require one step forward and one step back in the supply chain, referred to as a "one up, one down" system (FSANZ, 2017). All primary processors of animal material are required to have a risk management program (MPI, 2018). This is registered with the Ministry for Primary Industries (MPI) and sets how hazards and other risk factors will be identified, controlled and eliminated. This also involves setting out processes for record-keeping and product recall. Current traceability requirements explicitly for dairy include: the identification of products for export including relevant information such as date of manufacture, quantity, and sub-lot range; as well as keeping records of raw milk suppliers, their locations and the amount of milk supplied (Dairy Traceability Working Group, 2014).

Following the New Zealand government's enquiry into the 2013 whey protein botulism scare, the Dairy Traceability Working Group was established. This group soon proposed several extensions to traceability requirements in the dairy industry. The most significant of these revolves around the sharing of information. They propose key data elements should be able to be presented to MPI within 24 hours of a lawful trace back request. This data should be sufficient to allow the trace back and tracking forward of relevant dairy products (Dairy Traceability Working Group, 2014). This time limit seems short in the context of the time Fonterra took to locate affected product during the botulism scare, but is actually quite generous when we consider the potential impacts of dairy contaminations reaching consumers.

We develop a model for the flow of value though several stages of a supply chain. Initially, in Chapter 2 we incorporate product testing only, investigating the impacts of product loss at various stages of the supply chain and how this affects overall production. In Chapter 3 traceability parameters and effects are incorporated into the model in order to investigate the potential value of a good traceability system. By analysing the results of simulations 
under various scenarios we are able to estimate the value or the effect of a good traceability system on the output of the production chain.

We specifically apply these models to the dairy industry in New Zealand. Choosing parameter values to simulate value flow through several early stages of the supply chain for a major New Zealand dairy company. Accounting for $29 \%$ of export dollars coming into the country, the dairy industry is very important to New Zealand's economy (Ballingall \& Pambudi, 2017). Analysis of several scenarios, both with and without traceability, allows us to identify where testing and traceability systems have the greatest impact. Results of this analysis show there is significant value attributable to a good traceability system, due to precisely identifying product that needs to be removed. Separately, traceability has a larger impact when the traceability system influences the acceptance of milk arriving at the factory. The largest impact however is seen when a traceability system is active across all the stages of the supply chain modelled.

In support of this traceability modelling, in Chapter 4 of this thesis, we use an event study technique to examine how Fonterra is impacted financially by certain shocks. We analyse the impact of several product recalls as well as adverse weather events and global demand shocks for comparison. How the stock market reacts to recalls of varying size, scope and resolution time has particular importance for designing traceability systems. The response of share price returns can help to determine what aspects of a recall, and how it is managed, may have the largest impact, aiding the identification of particular areas of vulnerability and importance.

Good traceability is about reducing the consequences if a recall or withdrawal is necessary (Dupuy et al., 2005). Indeed it will aid in making the decision regarding whether a consumer level recall level is necessary. The results presented in this thesis highlight the importance of managing the recall process well, supporting the need for good traceability standards in the New Zealand dairy industry. 


\section{Chapter 2}

\section{Modelling New Zealand Dairy Production: From the Farm to the Factory}

\subsection{Introduction}

Dairy products are an important part of the western style diet and are becoming increasingly important as part of the Asian diet as well (Tsuda et al., 2000). Milk is a valuable source of essential nutrients, and often forms a large part of a young child's diet. Because of this, the dairy industry is particularly vulnerable to contamination scares. The 2008 melamine contamination of infant formula in China (Chan et al., 2008), the Fonterra botulism scare in 2013 (WPC 2013 Response Team) and the 2015 poisoning threat to infant formula in New Zealand (New Zealand Herald, 2015) are all examples of this. The 2013 botulism scare in particular, highlights the need for fast accurate testing and identification of substandard product. New Zealand has a reputation as a world leader when it comes to the production and export of dairy products (Jiang \& Sharp, 2014). The dairy industry currently accounts for $29 \%$ of export dollars coming into the country (Ballingall \& Pambudi, 2017). The amount of time taken to confirm the source of the contamination in the botulism scare of 2013, risked not only Fonterra's reputation but New Zealand's reputation as an exporter as well. 
The aim of this chapter is to develop a useful model for the early stages of the dairy supply chain, which we can use to investigate the overall effect of testing regimes and failure rates on output value. We develop a model for the flow of milk from the farm to the factory and incorporate testing to investigate its effect on product output. We aim to identify points in the supply chain where loss of product has the biggest impact on overall output value, in order to better manage and prioritise prevention or intervention schemes. Following this introduction, Section 2.2 discusses existing literature surrounding supply chain modelling in the dairy industry. Section 2.3 then gives an overview of the dairy industry in New Zealand. The section covers aspects of specific importance to the New Zealand dairy industry and discusses some aspects of testing dairy products. In Section 2.4 we introduce the stages of the model and develop a system of differential equations to model the value of milk in each stage over time. From the equations, we derive a set of Markov chains to reflect the stochastic nature of dairy production and contamination detection. Section 2.6 further develops the stochastic model and parameter estimation to incorporate more aspects of the dairy production process. Finally, we discuss the results and implications of this work, along with the limitations and potential for future research. The focus will be on the value of the milk, from which we can deduct costs associated with testing.

\subsection{Dairy Production and Supply Chain Modelling}

Supply chains have been modelled using many different algorithms and tools. These can be grouped into categories such as stochastic models, mathematical programming models, heuristic techniques and simulation (Biswas \& Narahari, 2004). Supply chain networks, in dynamic and stochastic environments are often considered as discrete event dynamic systems. Such systems include Markov chains, Petri nest and queuing network models (Raghavan, 1998).

Milk is rich in a variety of essential nutrients (Steijns, 2001), and the worldwide market for dairy and milk based products continues to grow (Huffman \& Harper, 1999). Along with this growth come increasing food safety issues, 
with consumer perception also becoming increasingly important (Etzel et al., 2012). Most of the existing research done on modelling dairy supply chains focuses on optimising some aspect of production. A large proportion of these studies propose some form of mathematical programming model, with an objective function they aim to minimise or maximise given certain constraints that must be met.

Doganis \& Sarimveis (2007) develop a mixed integer linear programming (MILP) model with the aim of minimising the variable costs associated with production in a single yogurt production line. Their model accounts for standard production scheduling constraints such as inventory limitations an machinery capacities. However, applicability of the model is limited to one single production line.

The problem of scheduling in a yogurt production line is addressed in Doganis \& Sarimveis (2008b). This time however a system involving multiple production machines in parallel is considered. The methodology incorporates features that allow it to tackle industry-specific problems, such as multiple due dates, job mixing and splitting, machine speeds, batch sizes and changeover times dependent on production sequences, as well as costs. The model is applied in a Greek dairy company, where it is found to greatly reduce production costs. This model is extended by Doganis \& Sarimveis (2008a) to include product shelf life.

Kopanos et al. (2009) also study a scheduling problem in a yogurt producing plant. They propose a MILP model, imposing timing and capacity constraints over several stages of production. Their framework optimises sequence-dependent setup times and costs, however only the packaging stage is involved in the problem they consider.

Bilgen \& Dogan (2015) develop an optimisation model of multistage production planning in the dairy industry. They formulate a MILP model, incorporating dairy production characteristics such as shelf life, storage, batch size limitations, production speeds and setups, and conservation of product flow. The model is applied to a leading dairy company, with the goal of maximising total profit and defining the quantity of product processed at each stage in order to achieve this.

One common supply chain modelling method that seems to be missing from 
dairy supply chain literature is simulations using Markov processes. Markov chains have commonly been used to simulate production and assess the likely impact of disruptions, interventions, or other changes how the supply chain is managed.

Song \& Zipkin (1996) explore an inventory-control model with periodic review. Assuming that orders progress though the supply chain randomly over time, they use a Markov chain model that is general enough to accomodate various disruptions and lead times. Similar to dairy production, they assume the supply system is exogenous, namely it is independent of demand. For convenience time and space are modelled as discrete, and all data as stationary in time. Their simulations suggest that a base-stock policy is optimal if there is no fixed cost, while a state dependent policy is optimal otherwise. Because of the generality of the model their result is applicable to a wide rage of inventory problems involving disruptions.

Horvath et al. (2005) use a Markov chain approach to investigate the potential damage retailers may experience as a result of uncertainty, variance and systemic shock associated with reverse logistics. They provide a useful tool for retail management, however only a single retailer was used for verification for the model, which limits how well the utility of the model can be assessed in context.

Tomlin (2006) analyse optimal dual-sourcing strategies in a single-product setting, with reliable and unreliable suppliers. Unreliable supply is modelled using a discrete time Markov chain and the optimal combination of supply from each source is investigated under various supply chain disruptions. Their model is not applied to the production of any particular product but provides a foundation for future research into the potential benefits of volume flexibility in many contexts.

G. Wang et al. (2008) construct a rotation model for grain storage in warehouses in China. They establish a Markov chain model and apply it with data from seventy national reserved grain depots of Henan Province. Their model is easily applicable to different kinds of storage warehouse.

Markov chain models and statistical approaches have been widely used in the study of blood supply chains and inventory systems for years (Beliën \& Forcé, 2012; Osorio et al., 2015). This is particularly interesting given many 
of the issues in collecting, storing and processing dairy products, for example the limited shelf life and fluid product, are shared by blood products. Blood products, such as plasma for example, are often mixed during processing. Brodheim et al. (1976) develop a model to derive regional allocations strategies for blood. They use a finite-state Markov chain to model the number of retention units in inventory immediately after each delivery. Boppana \& Chalasani (2007) develop Markov chain models to determine the optimal acquisition rate of blood, by hospitals, during emergencies. The model has a less fine focus than other literature, this makes it more appropriate for larger picture application however. Their proposed model is relatively simple, but for its proposed application in emergency planning. Their model is also only applicable to a single product at a time, meaning a seperate model needs to be developed for each product desire in each situation.

The stochastic model developed in this chapter fills a gap in the literature by applying Markov chains in the dairy industry. The similarities with the blood supply chain suggest this modelling technique could produce useful and interesting insights.

\subsection{Dairy in New Zealand}

As mentioned earlier, New Zealand is a world leader in the production and export of dairy products (Jiang \& Sharp, 2014). The New Zealand dairy industry is unique among major global producers in that the majority of its production is exported (Hutchinson, 2006). The industry is also mainly pasture based (Chand, 2006), and has earned a reputation for its low cost, high quality systems and technological expertise. There is still some room for improvement however (Jiang \& Sharp, 2014).

About $97 \%$ of New Zealand dairy farmers sell their milk through Fonterra Cooperative Group (Jiang \& Sharp, 2014). Cows are generally milked twice per day (Hogeveen \& Ouweltjes, 2002), and milk is collected from the farm in a tanker every one to two days (Jiang \& Sharp, 2014). Fonterra operates a national fleet of 525 tankers collecting from around 11,000 farms (Mason, 2014). The frequency of collection is generally dependent on the time of year, as milk production is seasonal. The amount of milk a farmer is allowed 
to supply to Fonterra is limited by the number of shares they own in the cooperative.

Figure 2.1 shows an overview of the flow of dairy products from the farm to the early processing stages at the factory. In the following paragraphs we describe relevant details of the New Zealand dairy industry in each stage of production present in this figure. We also discuss some of the testing that currently takes place.

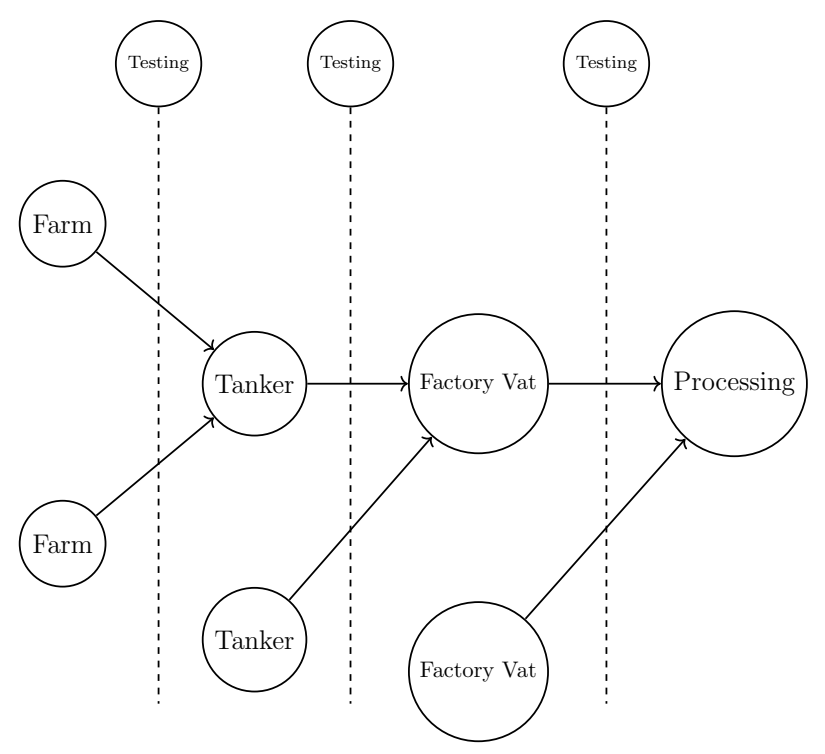

Figure 2.1: The overall picture of the dairy production chain, from the farm to initial processing at the factory.

\subsubsection{Processing In the Factory}

Aside from small quantities of on farm sales, the first steps in production required for all dairy products produced in New Zealand are separation, standardisation, and pasteurisation (Hudson et al., 2003; Western, 2011).

Typically a factory has a bank of several separators which feed into several silos for cream and skim milk. The Hautapu site, for example, has eight separators. This particular factory has the capability to process 4.1 million litres of milk per day. Each separator bowl has a volume of 50 litres and is capable of separating 33,000 litres every hour (Centrico, 1994).

For dairy-based infant formula, a typical processing plant has a separator 
capacity of 1,420,000 litres, made up of three separators, feeding into various product silos. Such a facility also has a standardisation capacity of 1,600,000 litres (Fonterra Co-operative Group, 2016a).

\subsubsection{Receiving Milk at the Factory}

The factory reception is the area where initial milk delivery takes place. The reception silos here need to have the capacity to accept the volume of milk that is potentially collected each day. The pumps used in the transport system are specifically designed to run at low speeds so as not to damage whole milk fat particles (Nuphlo Pumps Ltd, 2013).

The reception capacity of a typical processing site is about 675,000 litres (Fonterra Co-operative Group, 2016a). Keeping in mind that sites differ in capacity, given that Fonterra operates thirty three processing sites around the country we estimate a total reception capacity of 22,275,000 litres (= $33 \times 675,000)$.

\subsubsection{The Milk Tanker}

Fonterra's tanker fleet operates 24 hours a day, with a ten to twelve hour day shift involving three to six runs per tanker (McColl, 2011). Each run consists of, on average, five or six collection stops, taking about twelve minutes per collection, and a thirty minute turn around delivering to the factory (Dooley et al., 2005). There is a one to two hour turnover before the night shift starts with a similar pattern to the day shift (McColl, 2011).

Each milk tanker, truck and trailer unit can hold 28,800 litres of milk; 11,300 litres in the truck unit and 17,500 litres in the trailer (Dooley et al., 2005).

\subsubsection{On the Farm}

Rather than paying per litre of milk, Fonterra pays farmers based on the weight of milk solids they supply, measured in kilograms. Milk solids is the name given to the protein and fat content of milk, and a quantity of milk is often referred to in terms of kg MS (kilogram Milk Solids). 
A total of 1,614 million kg MS was collected by Fonterra in the 2014/2015 season ending in May 2015 (Fonterra Co-operative Group, 2015b). This equates to an average daily production of 4,421,918 $\mathrm{kg} \mathrm{MS}$, though this is skewed by the fact that very little production is taking place for the three months from June to August.

Milk is collected from suppliers on alternate days, unless daily collections are deemed appropriate based on milk volumes and vat capacity (Fonterra Co-operative Group, 2014b).

The capacity of an on-farm silo is based on each cow producing 25 litres of milk each day at the peak of the season. Fonterra currently requires their suppliers to have a minimum of 400 litres available at each collection (Fonterra Co-operative Group, 2014b). We will estimate an average collection amount per day, during the main season, based on herd size and cow output data.

Trends in developing high capacity milking parlours and automatic miking systems, have seen an increase in cow output, along with reduced manual labour on dairy farms. As these trends continue, further labour based barriers to farm expansion may be overcome (Hogeveen \& Ouweltjes, 2002). The average herd size has tripled over the last 30 seasons and is still increasing. For the 2014/2015 season, the average herd size was 419. The average output, per herd was 1,775,501 litres, containing $157,885 \mathrm{~kg}$ MS The number of dairy herds in New Zealand has been steadily declining since 1980, but has recently begun to increase again slightly, beginning in the 2007/08 season. This trend towards larger herd sizes in conjunction with the decreasing number of herds suggest a growing trend towards large dairy farm businesses. The overall number of individual cows continues to grow, so we are in no way seeing a decline in the dairy industry (LIC \& DairyNZ, 2015).

The price Fonterra pays farmers, in $\$ / \mathrm{kg}$ MS, is calculated based on the Global Dairy Trade prices for whole milk powder, skim milk powder, anhydrous milk fat, butter and buttermilk powder. Because these prices are in US dollars, the exchange rate must be taken into account before Fonterra subtracts the lactose cost and the cash and capital cost (NZX Agri et al., 2014). The farmgate milk price is the basic rate fonterra pays farmer for every kilogram of milk solids they provide. For the 2014/2015 season the farmgate 
milk price was $\$ 4.40 / \mathrm{kg}$ MS (Fonterra Co-operative Group, 2015a). We will use the price of $\$ 4.40 / \mathrm{kg}$ MS as the value for milk in our simulations. This price takes into account fixed costs such as transport and manufacturing as well as allowing for appropriate returns on investment (Fonterra Co-operative Group, 2017a). The 2014/2015 farm gate milk price was also relatively low, meaning if anything we underestimate the benefits of traceability.

\subsubsection{Testing}

The Fonterra supplier's handbook lists at least nine contamination types to be tested for along with with general quality grading and organoleptic (sensory) assessment, though only two of these tests are conducted upon tanker collection every time (Fonterra Co-operative Group, 2014b). Testing costs are generally less than 10 cents per test (Shitandi \& Kihumbu, 2004).

Fonterra milk tankers generally accept $99.99 \%$ of milk presented at the farm. Warm milk is the most common reason for rejection at this point. Temperature affects the quality of the milk and influences the growth of potentially harmful microorganisms (Akkerman et al., 2010). Fonterra's milk tankers are not refrigerated, meaning if the milk is not cold enough at the time of collection it may not arrive at the factory in an acceptable condition. There is also the possibility that the milk has not been chilled fast enough following milking and some quality degradation has already taken place.

Testing of milk upon collection can also improve the quality of the milk supplied. Gorton et al. (2006) identified a drop in milk rejection rates in Moldova between 1999 to 2003 from 4\% to 1\%, following the introduction of a collection and testing system holding individual suppliers accountable for the quality of the milk supplied.

\subsection{Developing the Model}

Identifying the stages of the supply chain where loss of product has the most impact can help a company prioritise quality control programmes. This aids in the reduction of overall product loss, both day to day and in the case of contamination events or where goods are otherwise compromised. We 
aim to develop a useful model for the flow of product in the dairy supply chain incorporating potential loss of product due to issues detected in quality control tests. While the overall output value is always reduced by testing, it is irresponsible and unrealistic for a company not to allow for loss of product in this manner. Testing is required to detect milk that is not fit for use. The focus of our modelling simulations is therefore on identifying where such losses have the largest impact on overall production value.

There are three possible points for testing, each before mixing together milk from different sources. As shown in Figure 2.1, these are between the farm vat and the milk tanker, between the tanker and the milk reception silo at the factory, and between the reception silo and entering processing. Milk may be declared unfit and therefore rejected for many reasons which differ at each stage. Each reason for rejection requires specific test methods. Milk being too warm for collection is one reason it may rejected by a milk tanker. Most other reasons for rejection involve some form of contamination, though his may be chemical, biological or larger foreign matter such as metal of plastic. We keep the concept of 'bad milk' fairly broad in this thesis, including all milk that could be is rejected for any reason. Not all 'bad milk' is necessarily detected however which is another reason to test at multiple stages, aside from the possibility of new reasons for rejection arising.

The parameters we will use in this model are summarised in Table 2.1. We make the assumption that any costs associated with the care and milking of cows is the responsibility of the farmer and do not influence our model.

\subsubsection{The Deterministic Model}

Initially we will develop a set of differential equations to model the basic situation. The resulting deterministic model will be a useful starting point when we construct the stochastic model. It will also assist in validating the simulation results. Finding the equilibrium values in each stage of production in the deterministic model will provide us with good initial values from which to begin our stochastic simulations.

Tanker Collection The flow of milk into and out of the milk tanker stage is depicted in Figure 2.2. In transferring the milk from the farm's vat to the 


\begin{tabular}{|c|l|c|}
\hline Parameter & Description & Units \\
\hline$V$ & Amount of Milk collected from an on-farm vat & $\$$ \\
\hline$\Phi$ & Frequency of collection attempts & Collections per day \\
\hline $\mathcal{X}$ & Frequency of delivery to factory & Deliveries per day \\
\hline$\Psi$ & Frequency with which milk enters processing & Silos per day \\
\hline$\Omega$ & Frequency of process exit & Units per day \\
\hline$E_{T}$ & Cost of testing milk at collection site & $\$$ \\
\hline$E_{F}$ & Cost of testing milk upon delivery & $\$$ \\
\hline$D_{F}$ & Cost of disposing of rejected milk & $\$$ \\
\hline$E_{P}$ & Cost of testing prior to processing & $\$$ \\
\hline$D_{p}$ & Cost of disposing of rejected silo milk & N/A (Probability) \\
\hline$\alpha$ & Probability of acceptance by tanker & N/A (Probability) \\
\hline$\beta$ & Probability of passing all tests upon delivery & N/A (Probability) \\
\hline$\gamma$ & Probability of passing pre-processing tests &
\end{tabular}

Table 2.1: A summary of each parameter used in our model and the associated units. Frequencies are represented by the capital Greek letters $\Phi, \mathcal{X}, \Psi$ and $\Omega$. Probabilities are represented by lowercase Greek letters $\alpha, \beta$, and $\gamma$. Testing costs are represented by an $E$ with a subscript for the associated stage. The subscript $T$ is associated with costs entering the tanker stage, $F$ the factory reception stage, and $P$ the processing stage. Similar notation is used for the disposal costs using a $D$. The vat value, given by $V$, uses units appropriate to the particular model simulation.

tanker, it becomes the responsibility of the dairy company and the value of that milk is added to the value in the tanker stage. This is also when the first test is applied, before mixing with any milk already in the tanker. The tanker gains the value of the milk, but loses the cost of any testing performed. If a test is failed before the milk is added to the tanker, the milk is rejected and no value is gained, the tanker still loses the cost of testing. After collecting milk from multiple farms the tanker will deposit its load at a factory, along with all the value associated with it.

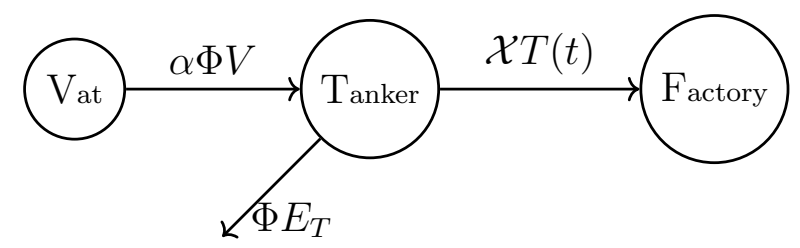

Figure 2.2: The flow of value into and out of the tanker stage. $T(t)$ is the value in the tanker stage at time $t$.

In any given period of time the tanker will make a certain number of col- 
lections and certain number of deliveries. In Figure 2.2 the frequency of collection, that is the number of collections attempts per day, is represented by $\Phi$ while delivery frequency is denoted by $\mathcal{X}$. The probability that the milk being collected passes all tests on site is denoted by $\alpha$, meaning $1-\alpha$ is the probability that a test is failed and the milk is rejected. $T(t)$ is the value contained by the tanker at time $t$. To avoid unnecessary complication, we assume that all vats contain the same amount of milk, and therefore the same value, $V$, represents the the value of the farm vat that is transferred to the milk tanker. The cost of the test is incurred independent of its results. This cost is assumed to be constant and is represented by $E_{T}$. If there is no testing prior to collection by the tanker, $\alpha=1$ as the milk cannot be rejected, and $E_{T}=0$ as there is no cost of testing. If milk is rejected, the co-operative does not pay the farmer for that milk, it is considered as if the milk was never supplied. The responsibility of disposing of milk rejected at this stage lies with the farmer (Fonterra Co-operative Group, 2014b).

We can represent the continuous, dynamic change in value of the tanker, $T$, with a differential equation:

$$
\frac{d T(t)}{d t}=\left(\alpha V-E_{T}\right) \Phi-\mathcal{X} T(t)
$$

Note the relationship to Figure 2.2, everything coming into the tanker stage is positive, while everything leaving is negative.

\section{Delivery to the Factory}

The next production stage is the reception silo at the factory, where the tanker deposits its load of milk. The flow of milk into and out of the factory reception silo is shown in Figure 2.3

The value in the factory reception stage is increased by $T(t)$ with each successful delivery, while the costs of testing, $E_{F}$, is removed. $\beta$ is the probability that milk passes all testing upon arrival at the factory.

Because at this stage the milk is the responsibility of the factory, there is also some disposal cost, $D_{F}$, associated with any milk that that is rejected upon arrival at the factory. 


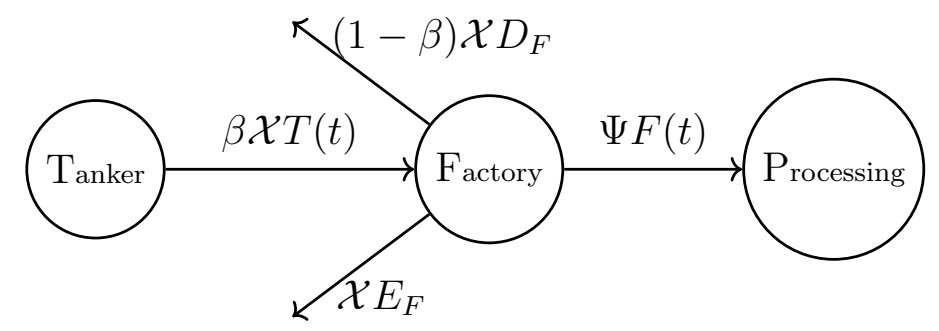

Figure 2.3: The movement of product into and out of the factory reception stage.

Because milk is being processed almost continuously, milk leaves factory storage in amounts dependent on how much is in the factory at time $t$. The frequency with which milk is sent on to the processing stage is denoted by $\Psi$. Equation 2.2 is the differential equation associated with this stage of production.

$$
\frac{d F(t)}{d t}=\beta \mathcal{X} T(t)-\mathcal{X} E_{F}-(1-\beta) \mathcal{X} D_{F}-\Psi F(t)
$$

As was the case the tanker stage, this equation mirrors the flow of product depicted in Figure 2.3. Product coming in from the tanker is positive, as it adds value to the stage, while product being passed on to the next stage, testing costs and disposal costs are negative, as they are value being removed from this stage.

\section{Processing}

Here we look at how the value contained in the processing stage is changing over time. Processing of milk begins with separation, followed by standardisation. After these steps every dairy product undergoes pasteurisation (Hudson et al., 2003; Western, 2011). The flow of value into and out of the production stage is depicted in Figure 2.4

The milk comes in from the factory storage vats at the same rate it leaves them in Equation 2.2, the value increases by this amount, $\Psi F(t)$ minus the value of testing conducted, $E_{P}$. The probability of accepting milk into this stage is denoted by $\gamma$. There will also be a disposal cost, $D_{P}$, for rejected milk. Value leaves this processing stage at the rate $\Omega P(t)$, to go on to the next stage in production. 


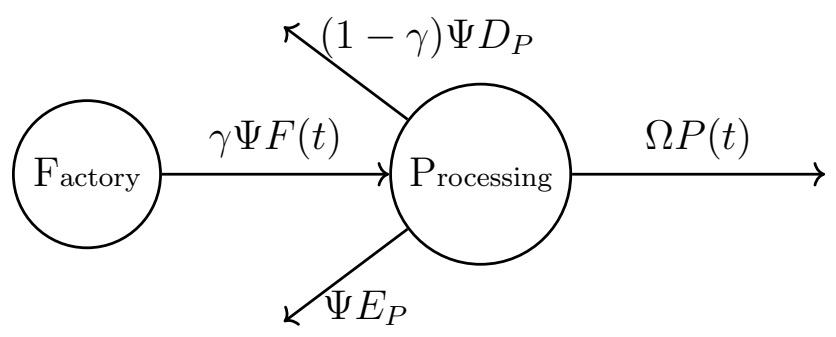

Figure 2.4: The rate of value flow into and out of the processing stage.

$$
\frac{d P(t)}{d t}=\gamma \Psi F(t)-\Psi E_{P}-(1-\gamma) \Psi D_{P}-\Omega P(t)
$$

Again, note the connection between Figure 2.4 and Equation 2.3. Value entering the processing stage is represented as positive, while value leaving is represented as negative.

\section{Equlibrium}

Now that we have a system of differential equations, we can solve for the value contained in each stage at equilibrium. The equilibrium point of the system occurs when all three first derivatives are zero.

$$
\begin{aligned}
& \frac{d T(t)}{d t}=\left(\alpha V-E_{T}\right) \Phi-\mathcal{X} T(t)=0 \\
& \frac{d F(t)}{d t}=\beta \mathcal{X} T(t)-\mathcal{X} E_{F}-(1-\beta) \mathcal{X} D_{F}-\Psi F(t)=0 \\
& \frac{d P(t)}{d t}=\gamma \Psi F(t)-\Psi E_{P}-(1-\gamma) \Psi D_{P}-\Omega P(t)=0
\end{aligned}
$$

Solving the tanker stage equation, first presented in Equation 2.1, for $T(t)$ gives:

$$
\begin{aligned}
\mathcal{X} T(t) & =\left(\alpha V-E_{T}\right) \Phi \\
\Longrightarrow T(t) & =\frac{\left(\alpha V-E_{T}\right) \Phi}{\mathcal{X}}
\end{aligned}
$$


This is the value gained with each successful milk collection, minus the cost of testing, all multiplied by the frequency of collection attempts and divided by the frequency of deliveries where product leaves this stage.

Solving the next equation, describing the flow of product through the factory reception stage (as originally presented in Equation 2.2), for $F(t)$ gives:

$$
\begin{aligned}
\Psi F(t) & =\left[\beta T(t)-E_{F}-(1-\beta) D_{F}\right] \mathcal{X} \\
\Longrightarrow F(t) & =\frac{\left[\beta T(t)-E_{F}-(1-\beta) D_{F}\right] \mathcal{X}}{\Psi}
\end{aligned}
$$

Substituting in the solution for the value of $T(t)$ at equilibrium, from Equation 2.4 gives

$$
\begin{aligned}
F(t) & =\frac{\left[\beta \frac{\left(\alpha V-E_{T}\right) \Phi}{\mathcal{X}}-E_{F}-(1-\beta) D_{F}\right] \mathcal{X}}{\Psi} \\
\Longrightarrow F(t) & =\frac{\beta\left(\alpha V-E_{T}\right) \Phi-\left[E_{F}+(1-\beta) D_{F}\right] \mathcal{X}}{\Psi}
\end{aligned}
$$

To find the equilibrium value in the processing stage we solve the third equation (originally presented in Equation 2.3) for $P(t)$ giving:

$$
\begin{aligned}
\Omega P(t) & =\left[\gamma F(t)-E_{P}-(1-\gamma) D_{P}\right] \Psi \\
\Longrightarrow P(t) & =\frac{\left[\gamma F(t)-E_{P}-(1-\gamma) D_{P}\right] \Psi}{\Omega}
\end{aligned}
$$

Substituting in $\mathrm{F}(\mathrm{t})$ at equilibrium, as given in Equation 2.6 gives us

$$
\begin{aligned}
P(t) & =\frac{\left[\gamma \frac{\beta\left(\alpha V-E_{T}\right) \Phi-\left[E_{F}+(1-\beta) D_{F}\right] \mathcal{X}}{\Psi}-E_{P}-(1-\gamma) D_{P}\right] \Psi}{\Omega} \\
\Longrightarrow P(t) & =\frac{\gamma \beta \Phi\left(\alpha V-E_{T}\right)-\gamma \mathcal{X}\left[E_{F}+(1-\beta) D_{F}\right]-\Psi\left[E_{P}+(1-\gamma) D_{P}\right]}{\Omega}
\end{aligned}
$$

This can be interpreted as all the material that is accepted into each stage, 
minus the costs associated with material testing and rejection, all divided by the rate it is leaving the processing stage.

\subsubsection{Product Flow in a Discrete Time Markov Chain Model}

Using Markov chains, we can derive realistic stochastic models based on the deterministic model developed in Section 2.4.1.

Let $X(1) \longrightarrow X(2) \longrightarrow \cdots \longrightarrow X(N)$ be a Markov chain. We move though each state in the chain over time, with a uniform time step, $\Delta t$, between each. The value in $X(t+1)$ depends solely on a set of transition probabilities, $p(X)$, that are functions of $X(t)$ and relevant parameters. When modelling a production chain each state is a vector containing the various locations of product throughout the system.

$$
X(t)=\left[\begin{array}{c}
x_{1}(t) \\
x_{2}(t) \\
\vdots \\
x_{i}(t)
\end{array}\right] \underset{p(X)}{\stackrel{\Delta t}{\longrightarrow}} X(t+1)=\left[\begin{array}{c}
x_{1}(t+1) \\
x_{2}(t+1) \\
\vdots \\
x_{i}(t+1)
\end{array}\right]
$$

The transition probabilities that determine the product movement each time step, will be unique to the specific production system being modelled

In this thesis, we model the flow of milk from the farm to the factory. Figure 2.5 shows the path milk takes from the farm to the factory, and where the event decisions occur. Milk passes through three locations after leaving the farm: tanker $(T)$, factory reception $(F)$ and processing $(P)$. The state of the Markov chain is thus described by a vector of three values:

$$
X(t)=\left[\begin{array}{l}
T(t) \\
F(t) \\
P(t)
\end{array}\right]
$$

$T(t), F(t)$, and $P(t)$ represent the value of product in each of the production stages at time $t$. The state of the Markov chain at a given time $t$ is the value of milk in currently each stage. Because the number of values each stage in 
$\mathrm{X}$ can take are finite, this is also a finite state Markov chain.

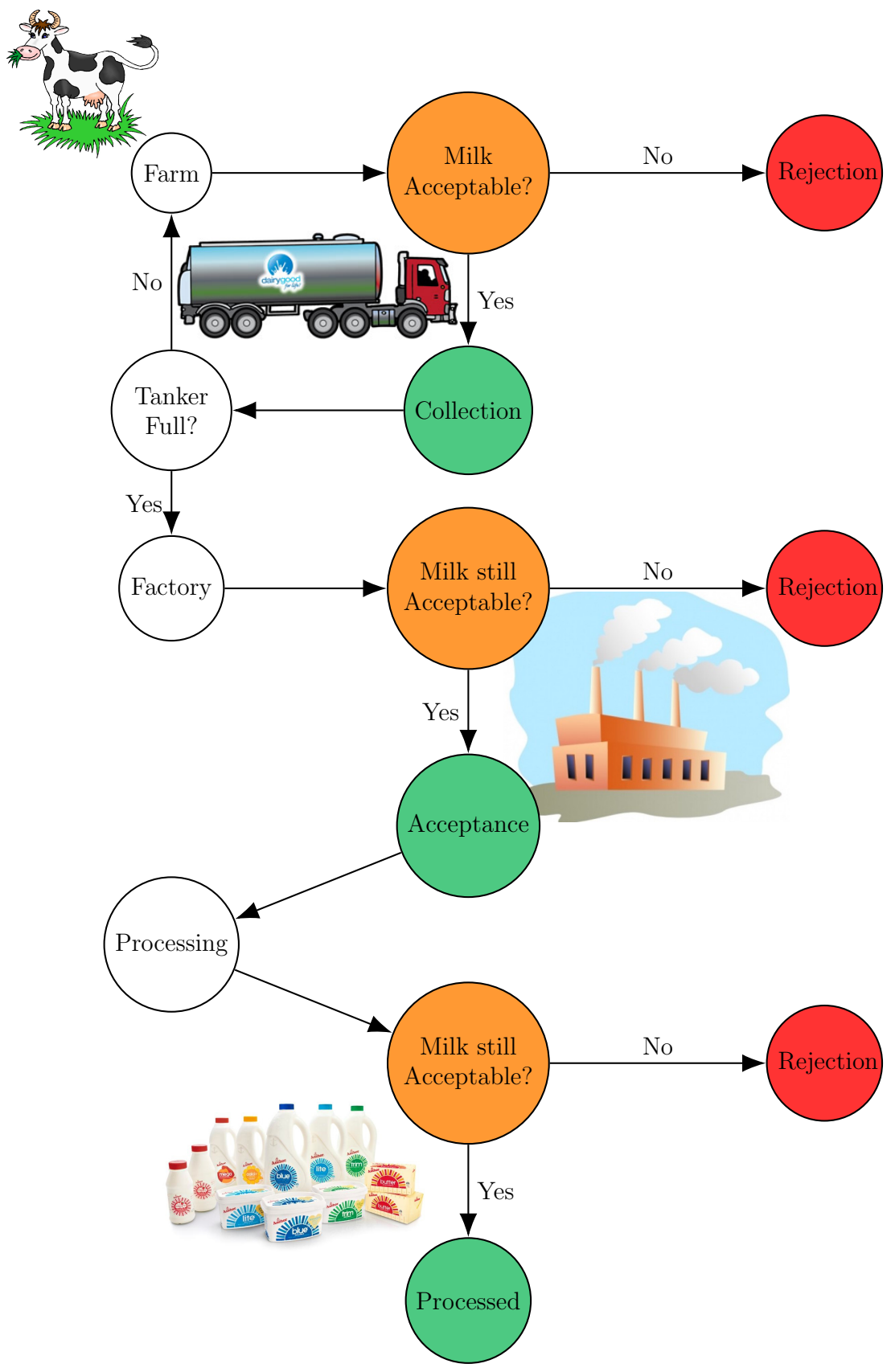

Figure 2.5: Flow chart showing the path milk takes from the farm to the factory and the decisions that are made along the way.

One strict requirement we must meet is that each set of transition probabilities adds to one. This is achieved via several modelling restrictions and techniques. First, the probability that there is no value change in a given 
stage is defined as one $t$ minus the sum of all the probabilities that some product movement does occur. For example the probability that milk in the tanker stage is only being transported in a given time step is given by one minus the probability that milk is being collected, rejected or delivered by a tanker. This definition is good as long as the probability of any movement adds to less than one, this we can ensure by choosing an appropriate time step. The frequency with product enters a stage, even when multiplied by the probability of acceptance (which is always less than or equal to one), will still likely result in a value greater than one. By choosing a value of $\Delta t$ that is small enough we can ensure the resulting probability is between zero and one.

Discrete time Markov chain models are defined on discrete time steps, where one unit changes state, or in this case location in the supply chain, at each step. The main issue with this type of model is that we need to be very careful choosing the size of the time step. Too small a time step and the computational burden becomes too great, too large a time step and we start to underestimate the changes. While a Continuous Time Markov Chain was considered, a discrete time Markov chain was chosen because of the way transitions, the events where milk moves between stages, are clearly defined and not instantaneous.

\subsection{The Single Event Model}

We start with a relatively simple model where only one event can take place at any point in time. The model operates with one tanker, one factory reception silo and one representative processing machine. We may lose some realism, but it provides a good place to start building the model from. The transition probabilities are given in Equation 2.9. The value of milk in the tanker, factory reception, and processing stages at time $t$ are represented by $i, u$, and $g$ respectively. This is the state of the Markov chain. The variables $j, v$, and $h$ denote the value change that takes place in each stage over a time step $\Delta t$. In this model the transition probabilities are constant; they always have the same value regardless of the value in each stage or the time of day. 


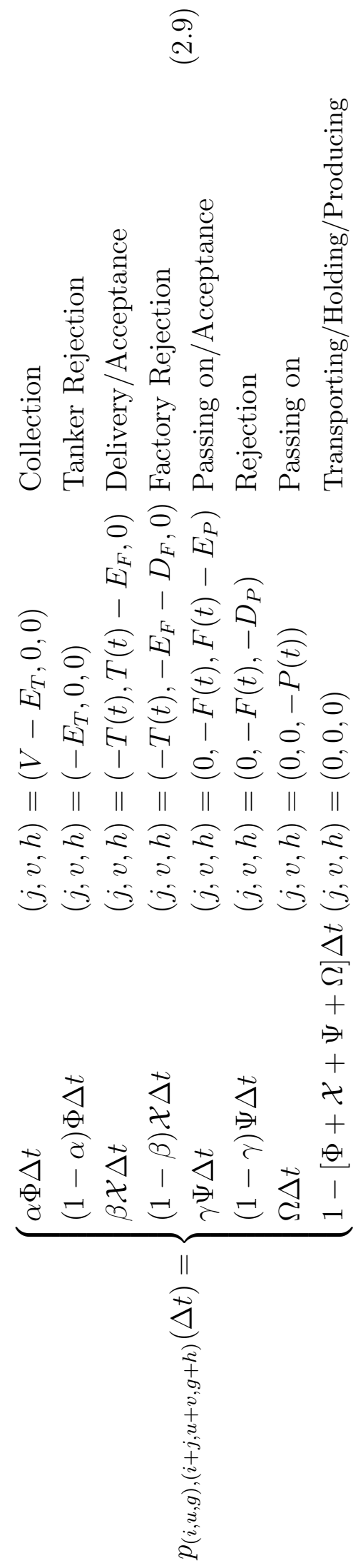


The Milk Tanker: There are four possible activities that a tanker can undertake in a given time step: milk collection, milk rejection, milk delivery, or transporting. Milk collection can only occur if no test results come back with an unsatisfactory result. The value contained in the tanker stage increases by the value of the milk collected, $V$, and is reduced by the cost of the testing, $E_{T}$. Milk rejection occurs if a test is failed. In this case the value contained in the milk tanker is decreased by the cost of the test, $E_{T}$. Milk delivery occurs when the tanker empties its load at the factory. The value contained in the tanker is reduced to zero. The total value currently contained by the tanker, $T(t)$, is delivered to the factory.

\section{The Factory Reception Stage:}

Delivery by the tanker implies either acceptance or rejection at the factory reception stage. In the case of rejection, the milk is rejected due to tests done upon the tanker's arrival at the factory. The cost of the test is lost $E_{F}$, along with the disposal cost $D_{F}$. The other possible event that can occur in this stage are passing on product for processing.

\section{Processing:}

Product being passed on by the factory reception stage is either accepted or rejected in the processing stage. Similar to the factory reception stage other possible activities include passing on product for further processing, but instead of just holding product, this is time spent in production.

\section{Transporting, Holding and Producing:}

Milk being transported by a tanker, held at the factory reception stage, or in the middle of processing, results in no change in the value of milk in any stage for that time step. The effect of each of these events is identical, therefore in this model they are represented as the same event even though they occur in different stages. 


\section{States of the Markov Chain:}

The total number of possible states depends on the amount of product that is allowed to move between stages in each time step, and the maximum capacity of each stage. For example, if the maximum capacity vector is given by $\left(T_{\max }, F_{\max }, P_{\max }\right)=(130,200,880)$ using a constant movement value of 10 units, the total number of states is given by $\frac{130}{10} \times \frac{200}{10} \times \frac{880}{10}=22880$. In this case, since we have no lower limit on how much material moves during each time step and no upper on limit stage capacities, the number of possible states is theoretically infinite.

\subsubsection{Parameter Values}

The initial parameter values, which we will discuss in this section, are compiled in Table 2.2. Initially we use a scenario where there is no risk of milk contamination with no testing implemented, and therefore the probability of acceptance at each stage is one. This scenario gives us a starting point from which to change parameter values as necessary. Thus we are left with only five parameters to estimate for now; farm vat collection value $V$, frequency of collection $\Phi$, frequency of delivery $\mathcal{X}$, frequency of process entry $\Psi$ and frequency of process exit $\Omega$.

\begin{tabular}{|c|l|r|}
\hline Parameter & Description & Value \\
\hline$V$ & Amount of Milk collected from an on-farm vat & $\$ 3050$ \\
\hline$\Phi$ & Frequency of collection a by tanker & 11970 \\
\hline $\mathcal{X}$ & Frequency of delivery to factory & 3990 \\
\hline$\Psi$ & Frequency with which milk enters processing & 311 \\
\hline$\Omega$ & Frequency of production & 346 \\
\hline$E_{T}$ & Costs of testing milk at collection site & 0 \\
\hline$E_{F}$ & Cost of testing milk upon delivery & 0 \\
\hline$D_{F}$ & Cost of disposing of unwanted milk & 0 \\
\hline$E_{P}$ & Cost of testing prior to processing & 0 \\
\hline$D_{P}$ & Cost of disposing of unwanted milk at factory level & 0 \\
\hline$\alpha$ & Probability of acceptance by tanker & 1 \\
\hline$\beta$ & Probability of passing tests upon arrival at factory & 1 \\
\hline$\gamma$ & Probability of passing pre-processing tests & 1 \\
\hline
\end{tabular}

Table 2.2: Parameter values in the no risk scenario. All frequencies are the average number of occurrences per day. 
The proportion of milk solids per litre of milk varies throughout the year. Table 2.3 shows the average milk production per cow per day, and per herd per day, by month from June 2014 to May 2015. The average kg MS is also given for each month. Average production values are calculated for peak production season between August and October and the whole of the main production season from August to April. May to July is the off season, generally only farms with special winter milk contracts are producing during these months, which may make the data during this period less reliable (LIC \& DairyNZ, 2015)

\begin{tabular}{|c|c|c|c|c|c|c|c|}
\hline & \multicolumn{6}{|c|}{ Production per day } & kg MS \\
\hline & \multicolumn{4}{|c|}{ per cow } & \multicolumn{2}{|c|}{ per herd } & per litre \\
\hline Month & Litres & Milk fat kg & Protein kg & kg MS & Litres & kg MS & \\
\hline June & 17.26 & 0.83 & 0.66 & 1.49 & 7231.94 & 624.31 & 0.0863 \\
\hline July & 18.34 & 0.87 & 0.72 & 1.59 & 7684.46 & 666.21 & 0.0866 \\
\hline Aug & 22.01 & 1.04 & 0.84 & 1.88 & 9222.19 & 787.72 & 0.0854 \\
\hline Sep & 23.50 & 1.08 & 0.89 & 1.97 & 9846.50 & 825.43 & 0.0838 \\
\hline Oct & 23.66 & 1.08 & 0.90 & 1.98 & 9913.54 & 829.62 & 0.0836 \\
\hline Nov & 21.33 & 1.01 & 0.82 & 1.83 & 8937.27 & 766.77 & 0.0857 \\
\hline Dec & 20.12 & 0.96 & 0.78 & 1.74 & 8430.28 & 729.06 & 0.0864 \\
\hline Jan & 17.41 & 0.86 & 0.67 & 1.53 & 7294.79 & 641.07 & 0.0878 \\
\hline Feb & 15.30 & 0.80 & 0.61 & 1.41 & 6410.70 & 590.79 & 0.0921 \\
\hline Mar & 13.19 & 0.74 & 0.56 & 1.30 & 5526.61 & 554.70 & 0.1003 \\
\hline Apr & 12.24 & 0.72 & 0.56 & 1.28 & 5128.56 & 536.32 & 0.1045 \\
\hline May & 13.05 & 0.74 & 0.59 & 1.33 & 5467.95 & 557.27 & 0.1019 \\
\hline \multicolumn{8}{|c|}{ Peak (Aug - Oct) } \\
\hline Ave & 23.05 & 1.06 & 0.87 & 1.94 & 9660.74 & 807.59 & 0.0842 \\
\hline \multicolumn{8}{|c|}{ Main Season (Aug - Apr) } \\
\hline Ave & 18.75 & 0.92 & 0.73 & 1.65 & 7856.71 & 693.49 & 0.0899 \\
\hline \multicolumn{8}{|c|}{ Full Season (Aug - July) } \\
\hline Ave & 18.11 & 0.89 & 0.71 & 1.61 & 7591.23 & 674.10 & 0.0903 \\
\hline
\end{tabular}

Table 2.3: Average daily milk production summary of the 2014/2015 season, based on data obtained from LIC \& DairyNZ.

Using the information in Table 2.3 we can estimate daily production values for the main season when most farms are producing, as well as just the peak season. Given there were 11970 herds supplying Fonterra in the 2014/2015 season (LIC \& DairyNZ, 2015), the average daily production from August to April can be estimated as $11970 \times 693.49=8,301,075 \mathrm{~kg}$ MS, equivalent to $92,336,763$ litres. The average daily production in just the peak season from August to October was $11970 \times 807.59=9,666,859 \mathrm{~kg} \mathrm{MS}$, the equivalent of $114,808,222$ litres of milk. In determining the parameters for this model 
only the main season, from August to April, when most farms are regularly producing milk is considered. We use the estimate that best applies to that time period for conversion between litres and $\mathrm{kg}$ MS, 1 Litre $\approx 0.0899 \mathrm{~kg}$ MS. Parameter values are estimated as follows below. Note in order to allow constant flow through the supply chain as necessary we require $\Phi \leq 3 \mathcal{X} \leq 33 \Psi \leq 33 \Omega$.

$V$ During the main milking season each farm is producing an average of $693 \mathrm{~kg}$ MS per day. Using a price of $\$ 4.40$ per $\mathrm{kg}$ MS, as detailed in Section 2.3.4, we can estimate the average value of milk produced by and collected from each farm per day as $V=\$ 4.40 \times 693=\$ 3050$.

$\Phi$ is the number of attempted milk collections by tankers each day. If milk is collected from each herd every day then $\Phi=11970$ collections per day.

$\mathcal{X}$ Given a milk tanker's capacity of 28,800 litres, we can estimate the number of farms a tanker can collect milk from in one run as between $\frac{28800}{7856.71}=3.66$ and $\frac{28800}{9660.74}=2.98$, or approximately three farms per collection run. If the farms are each collected from in one tanker run, we need $\frac{11970}{3}=3990$ tanker runs every day. Each run involves delivering to the factory once every run, so there will be $\mathcal{X}=3990$ deliveries each day.

$\Psi$ Fonterra has the capacity to process about seventy million litres of milk per day during the peak season (Fonterra, 2010). A typical paediatric processing site, in the infant formula production chain, has 3 silos of 225,000 liters capacity, for a total reception capacity of 675,000 litres. Given that Fonterra operates thirty three processing sites (Fonterra Cooperative Group, 2014a), each site would need to process $\frac{70000000}{33 \times 225000} \approx$ 9.43 silos of raw milk each day, or a total of 311 silos each day. So we set $\Psi=311$.

$\Omega$ The typical paediatric processing site, conducting the initial stage of processing for infant formula, has a bank of three separators feeding into two cream silos, three skim milk silos and two excess silos. This gives a total capacity of $2 \times 95,000+3 \times 350,000+2 \times 90,000=$ 
1, 420, 000 litres, and an average silo capacity of $\frac{1,420,000}{7}=202,857$. If the average capacity of a silo is passed on during every passing on event in the processing stage, we can estimate the frequency of this event as $\Omega=346\left(=\frac{70000000}{202,857}\right)$.

\section{Time Step Size}

As discussed in Section 2.4.2, the time step $\Delta t$ must be small enough to ensure that all of the transition probabilities, presented in Equation ?? sum to one. Each individual probability must also lie between zero and one. This means we require:

$$
\begin{aligned}
(\Phi+\mathcal{X}+\Psi+\Omega) \Delta t & \leq 1 \\
\Longrightarrow \Delta t & \leq \frac{1}{\Phi+\mathcal{X}+\Psi+\Omega}
\end{aligned}
$$

Substituting in the relevant parameter values gives:

$$
\begin{aligned}
\Delta t & \leq \frac{1}{11970+3990+311+346} \\
\Longrightarrow \Delta t & \leq \frac{1}{16617}=0.00006018 \text { days or } 5.199 \text { seconds }
\end{aligned}
$$

Based on this we choose time step of five seconds for our simulations, giving $\Delta t=0.00005787$. This means there will likely be a transfer of value between stages in almost every time step, but events occur frequently enough that realistic flow of material can happen.

\subsubsection{Single-Event Model Simulations}

Before we start analysing how testing affects the model, we need to check that the model gives realistic output in simulations. For each of the plots in this section, the initial values in each stage at time $t=0$ are estimated by Equations 2.4, 2.6, and 2.7, as developed in Section 2.4.1. These equilibrium values are where we would expect each stage to settle regardless of what initial 
values are chosen, using these initial values allows us to estimate average values and produce realistic plots without requiring a long 'burn-in' period (Flegal et al., 2008).

Initially, as we have not yet included any costs we can run our simulations using $\mathrm{kg}$ MS as our units. This is simpler to deal with as it is not subject to exchange rate and purchasing power fluctuations. Figure 2.6 shows a single simulation of the cumulative milk produced though our model over ten hours, along with a cumulative plot of the deterministic model. After ten hours this stochastic simulation, has produced 3,449,800 kg MS; the deterministic model reaches 3,456,300 $\mathrm{kg}$ MS. These values equate to 8,279,500 $\mathrm{kg}$ MS per day and $8,160,000 \mathrm{~kg}$ MS per day respectively. Given the collection rates seen in Section 2.3.4 and Table 2.3 and the absence of any product rejection, these values mirror the average production we would expect to see during the main milking season, from August to April.

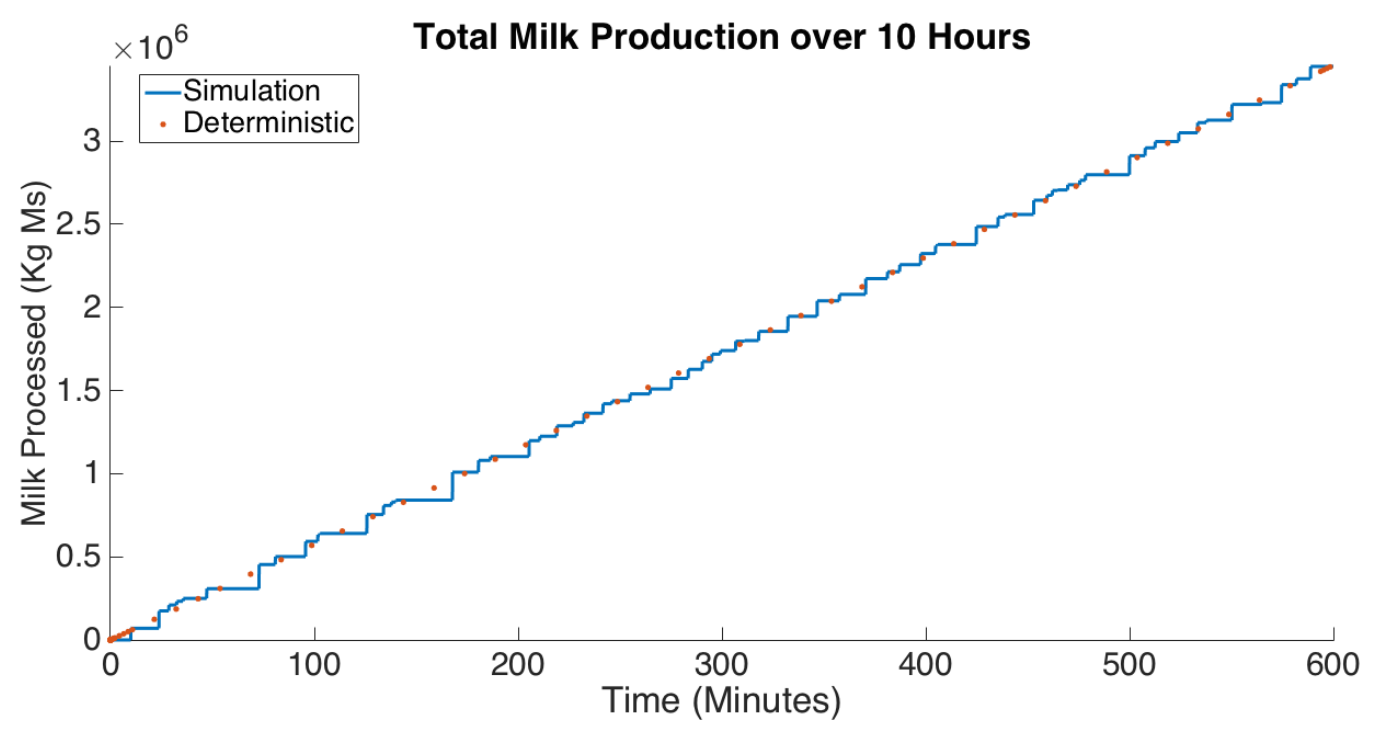

Figure 2.6: The amount of milk output after the three stages of processing in our model. Both a stochastic simulation and the deterministic model solution are shown over a ten hour period.

The amount of product contained in each stage at each point in time over a ten hour simulation period is shown in Figure 2.7. Here we can see some of the interaction between the three stages. The effect the tanker value has on the factory stage can be seen in the little increases as the tankers feed into the factory reception. The processing stage shows less frequent but larger increases as the the factory storage stage empties into it. Note, as 
mentioned in Section 2.5, this model does not account for limited capacities. The amount of product simulated in the processing stage occasionally exceeds what a factory can realistically contain at a given point in time.

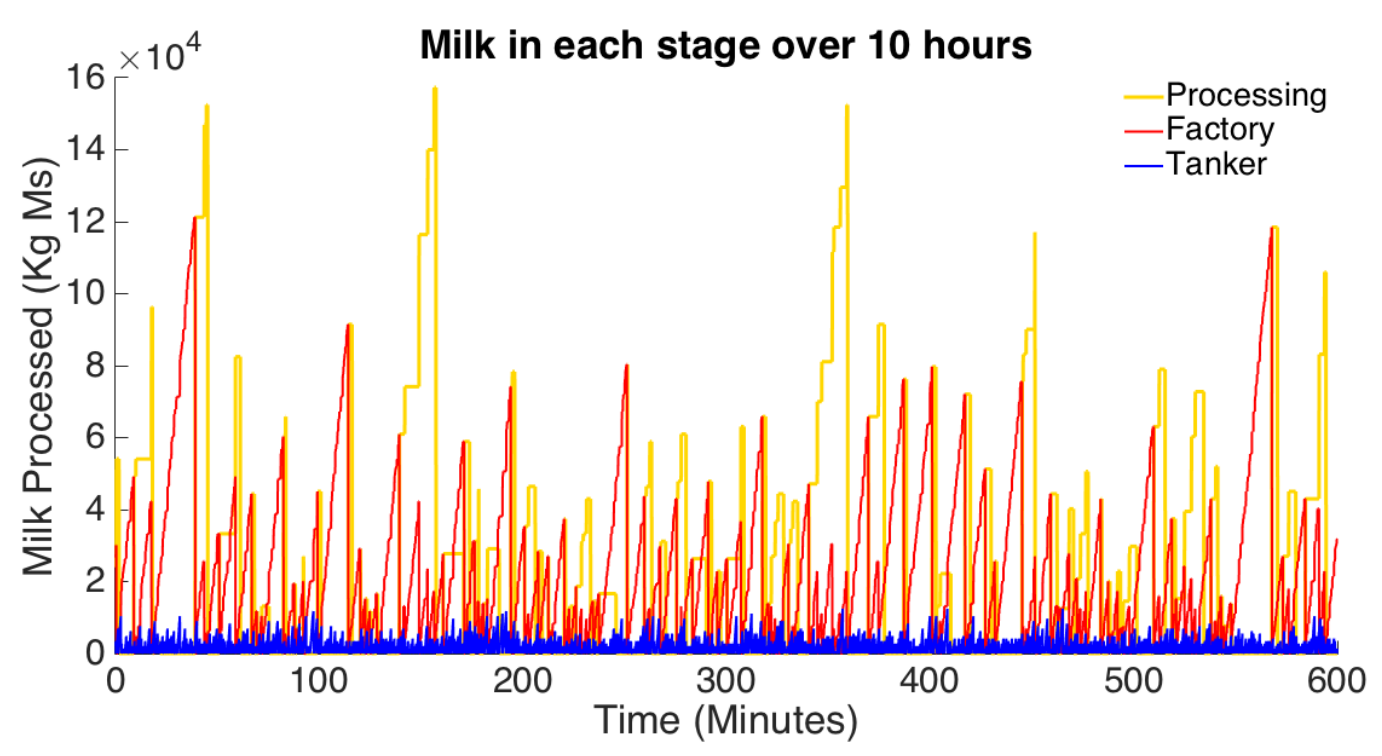

Figure 2.7: The amount of milk contained in each stage over a ten hour simulation.

Zooming in on an hour near the middle of Figure 2.7, shown in Figure 2.8, allows us to see the interaction between the tanker stage and the factory reception stage more clearly. The emptying of the tanker stage coincides with an increase in the factory stage as expected.

In this simulation the amount of milk in the tanker stage averages 1936.3 $\mathrm{kg}$ MS. The factory reception and processing stages each average 22,941 kg MS and 23,414 kg MS respectively. Using the current parameter values and Equation 2.4, the deterministic solution for the tanker stage, when in equilibrium, is $2079 \mathrm{~kg}$ MS. The values given by Equations 2.6 and 2.7 are 26,673 $\mathrm{kg}$ MS and 23,975 $\mathrm{kg}$ MS respectively. In the tanker case the difference is $142.7 \mathrm{~kg}$ MS or $6.86 \%$ of the expected value, over just this ten hour simulation. In the case of the factory reception stage the difference is $3732 \mathrm{~kg}$ MS or $13.99 \%$ while the difference in the processing stage is just $561 \mathrm{~kg}$ MS or $2.34 \%$. Keeping in mind that this is just a ten hour simulation there is still a large difference between how well each stage matches the expected equilibrium value, though they are all at least in the right order of magnitude. The tanker stage may match the equilibrium value so well because the value 


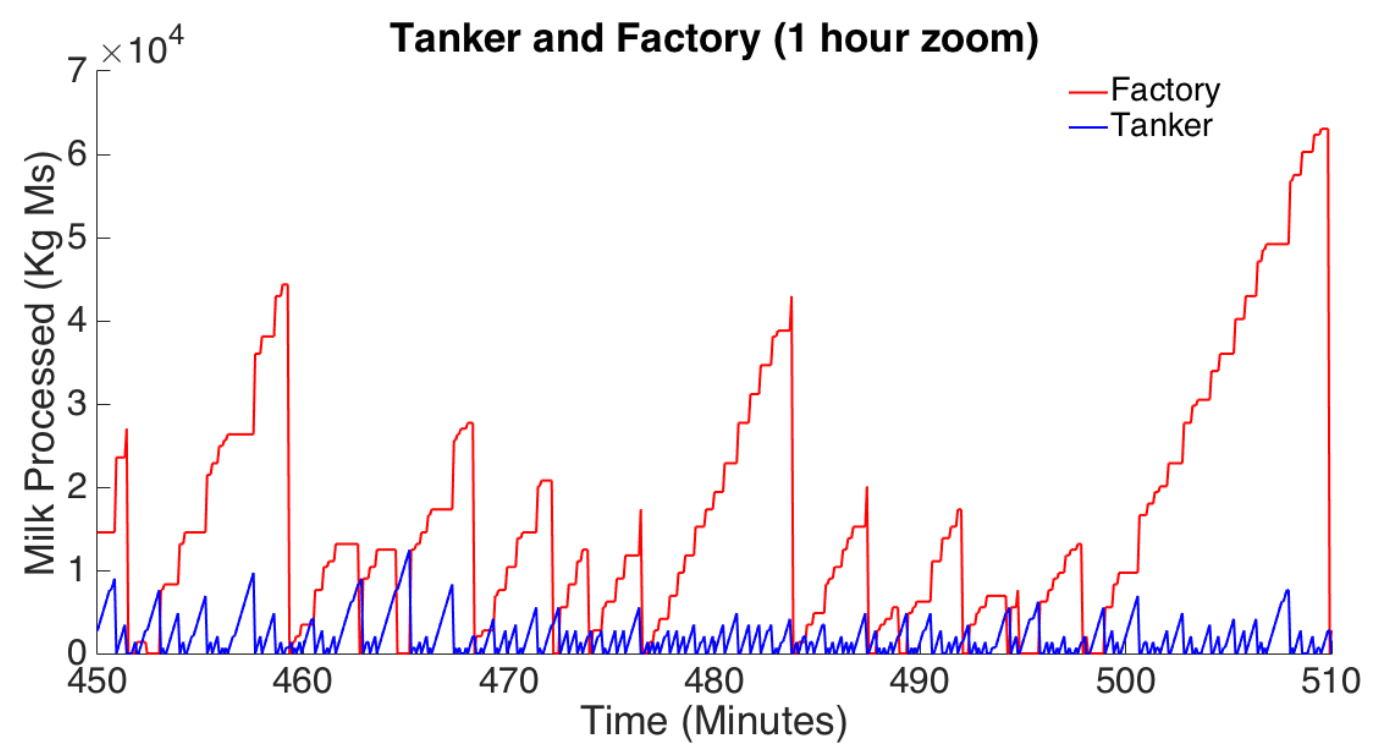

Figure 2.8: An hour from the middle of Figure 2.7 focusing on the simulation of the Milk Tanker and Factory Reception stages.

in the stage is regularly changing by small amounts. The other two stages change value less often, and change dramatically when they do change. The average amount of milk contained in each stage over twenty five and fifty days worth of simulation are given in Table 2.4.

\begin{tabular}{|l|r|r|r|}
\hline Stage & Deterministic Equilibrium & 25 day average & 50 day average \\
\hline Tanker & $2079 \mathrm{~kg}$ MS & $2074.89 \mathrm{~kg} \mathrm{MS}$ & $2076.24 \mathrm{~kg}$ MS \\
\hline Factory Reception & $26,673 \mathrm{~kg} \mathrm{MS}$ & $26,861.03 \mathrm{~kg} \mathrm{MS}$ & $26,851.49 \mathrm{~kg} \mathrm{MS}$ \\
\hline Processing & $23,975 \mathrm{~kg} \mathrm{MS}$ & $23,467.68 \mathrm{~kg} \mathrm{MS}$ & $23,689.76 \mathrm{~kg}$ MS \\
\hline
\end{tabular}

Table 2.4: The average value in each stage after simulations of 25 and 50 days along with the equilibrium values given by the deterministic model given in section 2.4.1.

Over sixty runs, equivalent to six hundred hours or twenty five days, the average amount of milk contained in the tanker stage is $2074.98 \mathrm{~kg}$ MS. This is much closer to the predicted equilibrium value of $2079 \mathrm{~kg} \mathrm{MS}$, a difference of just $4.02 \mathrm{~kg}$ MS or $0.19 \%$. The factory reception and processing stages average 26,861.03 kg MS and 23,467.68 kg MS. The difference between the factory simulation average and the deterministic equilibrium has significantly decreased as well to just $188.03 \mathrm{~kg}$ MS or $0.70 \%$. In the case of the processing stage, the average level is $507.32 \mathrm{~kg}$ MS below that of the deterministic equilibrium or $2.11 \%$. Interestingly this is very similar to the difference after 
a single simulation and may be due to the large volume of product that is potentially moving in and out of this each time step. Over 120 runs, or essentially 1200 hours or 50 days the tanker average is $2076.24 \mathrm{~kg}$ MS, the factory reception average is $26,851.49 \mathrm{~kg} \mathrm{MS}$, and the processor average is now $23,689.76 \mathrm{~kg}$ MS. The percentage differences from the deterministic equilibrium are now $0.13 \%, 0.67 \%$, and $0.12 \%$. All three stages now average closer to the deterministic equilibrium value as we would expect them to after more and more runs.

All three stages spend a significant amount of time empty in this model, to the point where the lower quartile for each stage is 0 . This is likely due to how a stage is totally emptied each time it passes product on to the next stage.

\subsection{Developing The Multi-Event Model}

The model in the previous section only allowed for one event to occur in the whole modelled supply chain in each time step. Also, because the volume of milk in each stage was changing so much each time material moved between stages, we had very large, sudden peaks and troughs. This was particularly noticeable in the factory reception and processing stages, and is something we improve upon with the model in this section.

In this model the amount of product transferred in each collection, delivery or passing on event is now a defined constant. We also define limited capacities for each stage of production and modify the existing transition probabilities and transition quantities to reflect these capacities. The transition probabilities are presented individually for three stages in this section. This means that events occurring at different stages can take place simultaneously during the same time step, hence we can can have multiple (up to three) events occurring in each time step as opposed to only one event in the previous model. The model remains time invariant, as the transition probabilities and quantities are independent of the time, but the probabilities now vary with respect to the value in each stage at time $t$. 


\subsubsection{The Milk Tanker}

The value in each tanker is still incorporated into one pool, so the value collected or delivered by one tanker is still added to, or deducted from, the tanker value pool, but tankers will add to or remove material from that pool individually. We define the capacity of one milk tanker as $C_{T}$, and the total capacity of the tanker stage as $N_{T}$

In this model we only allow at most one event, out of collection, rejection, delivery, or transporting, to take place in any given time step. In reality, as there are many milk tankers, two or more tankers may collect milk from different farms simultaneously. However in reality, these events also take time. In our model, it is possible for two events to occur in two adjacent time steps, and these time steps are necessarily quite small, this means we can closely mimic simultaneous events for different tankers t.

We describe the transition probabilities for the tanker stage, and their associated value changes, below. They are summarised in Equation 2.11 in Section 2.6.4 along with the transition probabilities for each of the other stages.

Collection. The probability that a milk tanker will attempt to collect milk varies with the amount already contained in the tanker stage. If $T(t) \geq N_{T}$, for some time $t$, all of the milk tankers are full and milk collection must be impossible for that time step. The probability of an attempted milk collection in time step $\Delta t$ is given by

$$
\begin{aligned}
& \frac{N_{T}-T(t)}{N_{T}} \Phi \Delta t \\
= & \left(1-\frac{T(t)}{N_{T}}\right) \Phi \Delta t
\end{aligned}
$$

As $T(t)$ approaches $N_{T}$ the probability of a collection attempt approaches zero. Recall that $\Phi$ is the number of attempted collections per day.

The probability that the tanker accepts the milk presented by a farm for collection is $\alpha$, the probability that a milk tanker will collect milk in a time step $\Delta t$ is thus given by:

$$
\alpha\left(1-\frac{T(t)}{N_{T}}\right) \Phi \Delta t
$$


The change in value in the tanker stage in this event is $V-E_{T}$, the amount provided by the farm vat minus the cost of testing it.

Rejection. If the milks fails any tests prior to collection it will be rejected by the tanker. The probability of the milk tanker rejecting the milk supplied at a farm is given by

$$
(1-\alpha)\left(1-\frac{T(t)}{N_{T}}\right) \Phi \Delta t
$$

Rejected milk is left at the farm, the cost of disposal is the responsibility of the farmer, however the tanker stage does not gain any value and still loses the cost of the tests conducted. This results in a change of $-E_{T}$.

Delivery. After collecting milk from multiple farms, the tanker will empty its load at a factory, transferring all of the value associated with it. The probability that a tanker will deliver to the factory is dependent on the amount milk in the tanker stage available to deliver. Because of this if there is no milk in any tankers, i.e. $T(t)=0$, the probability of a delivery in that time step is zero. The probability of delivery also increases proportionally with the value of milk contained in the tankers. Delivery is also not possible if the factory reception stage is full. The total capacity of the factory reception stage is given by $N_{F}$, thus if $F(t) \geq N_{F}$ for any given time step, delivery to the factory is not possible. Otherwise the probability of a tanker delivering milk to the factory in time step $\Delta t$ is be given by

$$
\frac{T(t)}{N_{T}}\left(1-\frac{F(t)}{N_{F}}\right) \mathcal{X} \Delta t
$$

Where $\mathcal{X}$ is the number of deliveries to the factory attempted each day. We denote the capacity of one milk tanker as $C_{T}$, thus the change in tanker value upon delivery is $-C_{T}$.

\subsubsection{The Factory Reception}

The next stage in the supply chain is the factory reception silo, where tankers deliver their milk. Fonterra has thirty three processing sites around the 
country (Fonterra Co-operative Group, 2014a), as with the tankers we will be treating these as part of the factory reception pool, but each site receives and passes product on individually. The capacity of one reception silo is defined as $C_{F}$. The possible events in the factory reception stage are collection, rejection, passing on for processing, or holding.

Each milk tanker has both a trailer and a truck compartment. Because these two compartments can be kept separate they can be accepted and rejected separately. Figure 2.9 shows a probability tree for how the probability of each acceptance and rejection combination is calculated. The rejection of a tanker compartment is denoted by $\mathbf{r}$, while $\mathbf{a}$ denotes its acceptance up to that point. We end up with three main possibilities; total acceptance, partial acceptance or total rejection. The probability that a tanker compartment is contaminated is not totally independent of the other compartment's status. There is the possibility that some milk from one of the farms collected from ends up in each tanker. For example if each tanker is collecting from three farms, there is a one in three chance that an overlapping collection is from the contaminated batch. The probability that one tanker compartment passes all tests, given that the other tanker compartment requires disposal, is given by $\varsigma$.

\section{Outcome/}

\section{Tank 1 Tank 2 Rejection Type Probability}

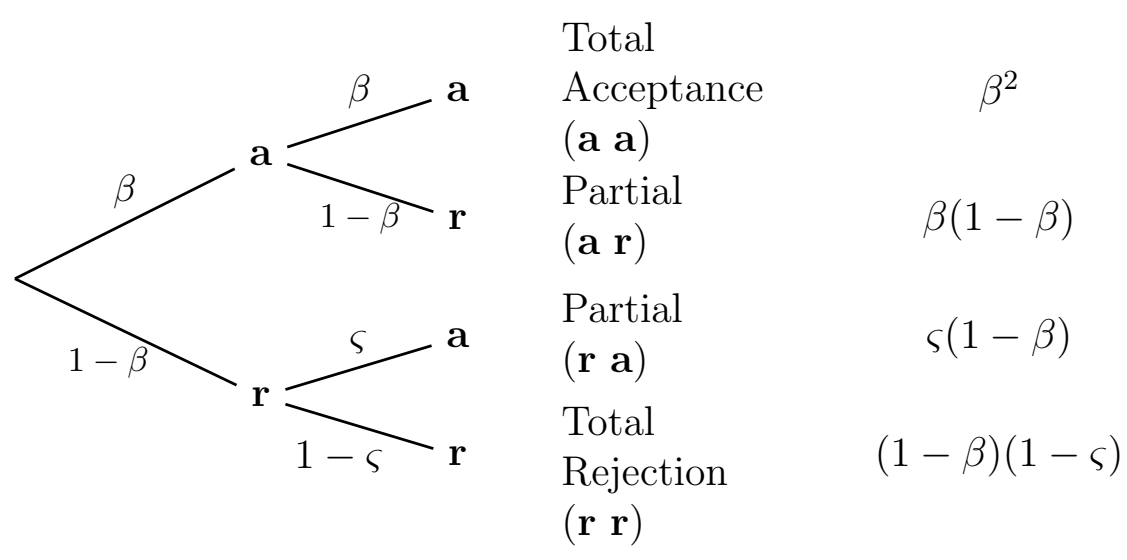

Figure 2.9: Probability tree showing the possible outcomes when milk is delivered to the factory. The rejection of a tanker compartment is denoted by $\mathbf{r}$, while $\mathbf{a}$ denotes acceptance. 
Each event and its associated affect on the value contained in the factory reception stage is described below. The transition probabilities are summarised in Equation 2.11.

Total acceptance. In the case of total acceptance no contamination is detected in either tanker compartment. Therefore the probability of a milk delivery being fully accepted is simply the probability of a delivery attempt multiplied by the probability that both compartments pass. The probability of a delivery attempt is given in Equation 2.10. If $\beta$ is the probability of a tanker compartment passing all tests conducted at this stage, the probability of total acceptance is given by

$$
=\beta^{2} \frac{T(t)}{N_{T}}\left(1-\frac{F(t)}{N_{F}}\right) \mathcal{X} \Delta t
$$

The value in the factory reception stage increases by the value that was delivered by the tanker, $C_{T}$, while the cost of testing, $E_{F}$, is subtracted from this value. The resulting total value change is $C_{T}-E_{F}$.

Partial acceptance. A partial acceptance implies that just one tanker compartment is accepted by the factory. This occurs with probability

$$
(1-\beta)(\beta+\varsigma) \frac{T(t)}{N_{T}}\left(1-\frac{F(t)}{N_{F}}\right) \mathcal{X} \Delta t
$$

This is the sum of the probabilities that the rejection occurs in either tank. The order of rejection has no effect in this model. Because only one tanker compartment is rejected, half of the milk that was delivered, $\frac{C_{T}}{2}$, is added to that currently contained in the factory reception stage. Half of the disposal cost, $\frac{D_{F}}{2}$, is also incurred due to the compartment that needs to be disposed of. The full testing costs, $E_{F}$, still apply. Thus the value change in the factory stage in the case of of a partial rejection is given by $\frac{C_{T}-D_{F}}{2}-E_{F}$.

Total rejection. A total rejection requires both tanks to be rejected due to the results of testing on arrival at the factory. The probability of this occurring is given by 


$$
(1-\beta)(1-\varsigma) \frac{T(t)}{N_{T}}\left(1-\frac{F(t)}{N_{F}}\right) \mathcal{X} \Delta t
$$

In this event the factory reception gains no value, and still loses the cost of testing, $E_{F}$, and disposing of, $D_{F}$, both tanker compartments. The total value change in this case is then $-E_{F}-D_{F}$.

Passing on. The other possible value changing event that can occur in this stage is passing on material to the initial processing stage. The probability of a 'passing on' event is dependent on the value of milk contained in the factory reception stage. If the factory reception stage is empty, $F(t)=0$, and no product can be passed on. Equivalently milk cannot be passed on to a full processing stage, defined by $P(t) \geq N_{P}$, where $N_{P}$ is the total capacity of the processing stage.

The probability that milk will be passed on for processing is given by

$$
\frac{F(t)}{N_{F}} \Psi \Delta t
$$

It is useful to note that this probability is a model approximation. In reality the rate of movement into the next stage is not so smoothly linear as many other factors can influence when milk is processed. This approximation however is adequate for its use in this modelled system. The capacity of one reception silo, denoted by $C_{F}$, is the amount that will leave the factory reception stage and move on for processing. The change in value when milk is passed on for processing is thus $-C_{F}$.

\subsubsection{The Processing Stage}

This next stage in our model represents the first stages of processing that every Fonterra dairy product undergoes. Similar to the other stages, the possible events in this stage are acceptance or rejection of incoming product, passing on product for further processing and/or packaging or, where no product value is entering or leaving this stage, producing. We describe each event and the effect it has on the value in the processing stage below. These 
transition probabilities are summarised in Equation 2.13, in Section 2.6.4.

Acceptance. When milk is passed on from the factory reception stage, it is accepted by the processing stage with probability $\gamma$. Thus the probability that all of the milk in a reception silo is accepted for processing in any given time step is

$$
\gamma \frac{F(t)}{N_{F}} \Psi \Delta t
$$

The value in the processing stage, in the event of acceptance, increases by the value transferred from the factory reception stage, the capacity of one reception silo $C_{F}$, and decreases by the cost of tests conducted prior to acceptance $E_{P}$. The total value change in this event is thus $C_{F}-E_{P}$.

Rejection. Milk passed on by the factory reception stage is rejected by the processing stage with probability $1-\gamma$. Thus probability of a rejection at the processing stage is

$$
(1-\gamma) \frac{F(t)}{N_{F}} \Psi \Delta t
$$

The processing stage does not gain any value in this case and loses the costs of any tests that were performed, $E_{P}$, along with the costs of disposing of the milk, $D_{P}$. Thus the total value change in the event of a rejection at this stage is $-E_{P}-D_{P}$.

Passing on. The probability that material is passed on from this processing stage is given by

$$
\frac{P(t)}{N_{P}} \Omega \Delta t
$$

There are no costs associated with passing material on to the next stage, but the value of material in the processing stage will decrease by $Q$, the value of milk that moves in each passing on event. 


\subsubsection{Summary equations and Stage Interactions}

The following equations summarise the probabilities and outcomes described in Sections 2.6.1, 2.6.2 and 2.6.3. The events that happen in each stage can affect the value in the other stages. For the purposes of this model, the value changes that occurring in each stage do not come into effect until all events taking place in a given time step are complete. Each time step begins with the vector $(T(t), F(t), P(t))=(f, i, u)$.

Equation 2.11 summarises the new transition probabilities for the milk tanker stage, the outcomes of which also affect the value in the factory reception stage, as denoted in the brackets. Any changes in value that happen in the tanker stage however, do not affect the probability of any events happening in the other stages within the same time step.

The factory reception transition probabilities are summarised in Equation 2.12 below. An acceptance or rejection of any kind at this stage, implies that there a delivery from the tanker stage, and the value in the tanker stage is changed accordingly. In the event the factory reception stage is passing on product, the processing stage must also be accepting or rejecting that product. This is denoted in the brackets on the right had side of Equation 2.12 , the value in the processing stage changes as appropriate.

The transition probabilities for the processing stage are given below in Equation 2.13. The events that occur in this stage also affect the value in the factory reception stage as denoted.

At the beginning of each time step we start with $(T(t), F(t), P(t))=(f, i, u)$. Once all of the events in all stages are complete these values are updated to $(T(t), F(t), P(t))=(f+g+h, i+j+k+s, u+v+w)$, as defined in the transition probabilities above.

Time step. To ensure that the probabilities in every stage all add to less than one we need to choose a small enough value for $\Delta t$. In the case of the tanker stage this means we require

$$
\left[\left(1-\frac{T(t)}{N_{T}}\right) \Phi+\frac{T(t)}{N_{T}}\left(1-\frac{F(t)}{N_{F}}\right) \mathcal{X}\right] \Delta t<1
$$




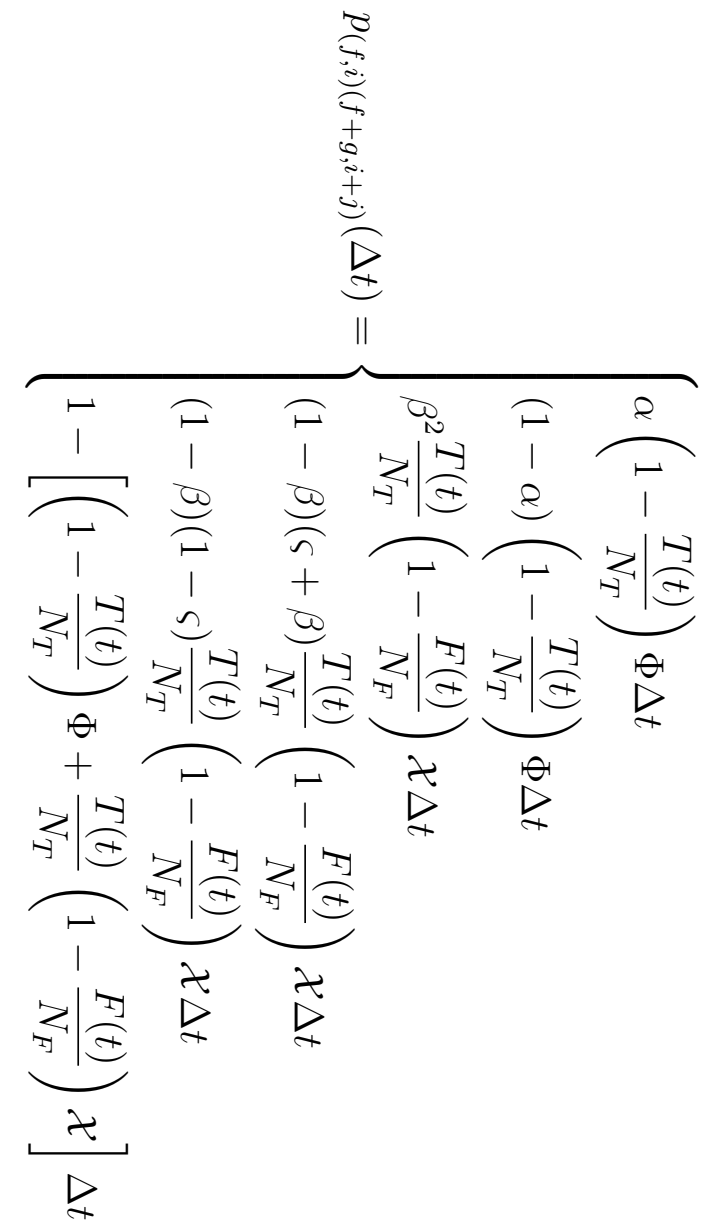

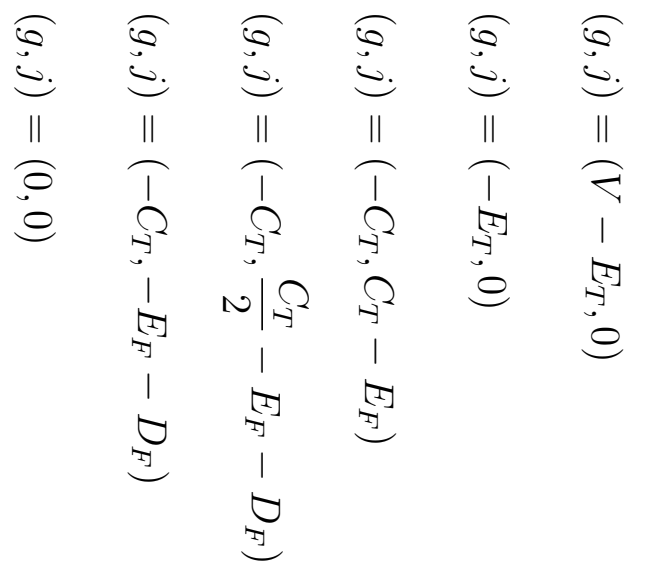

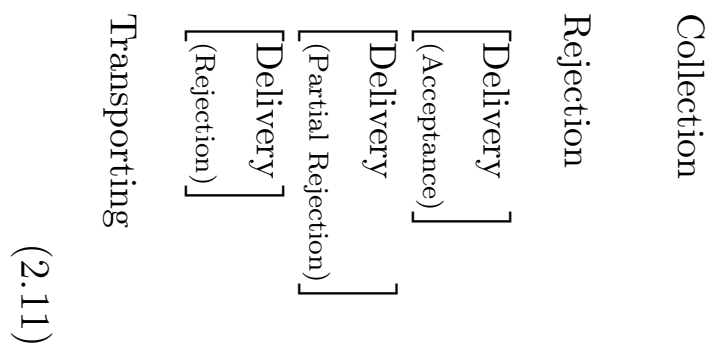




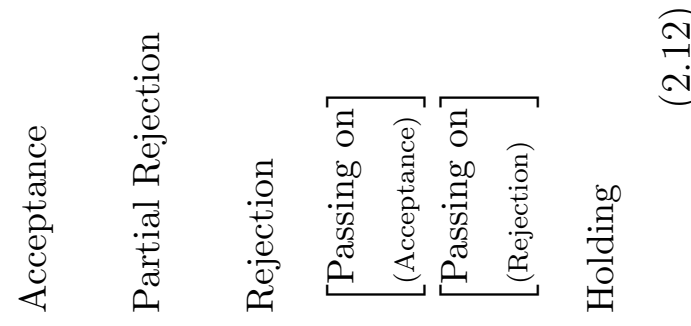

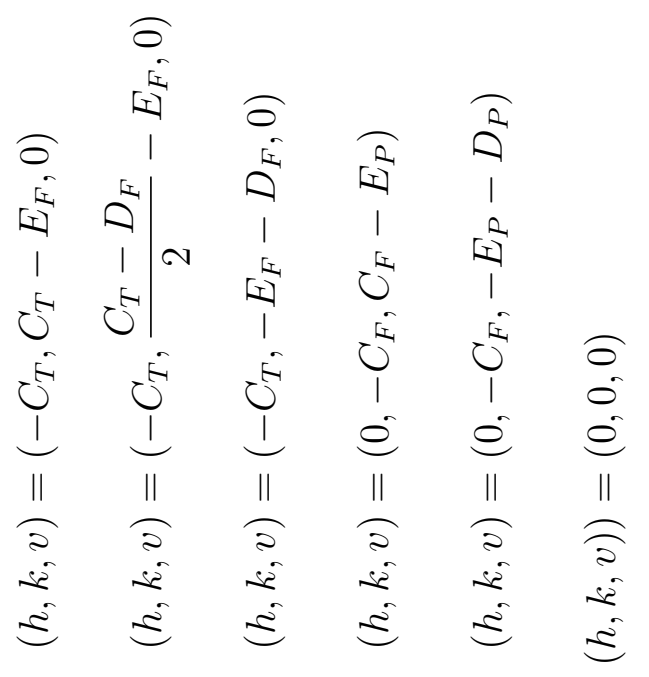

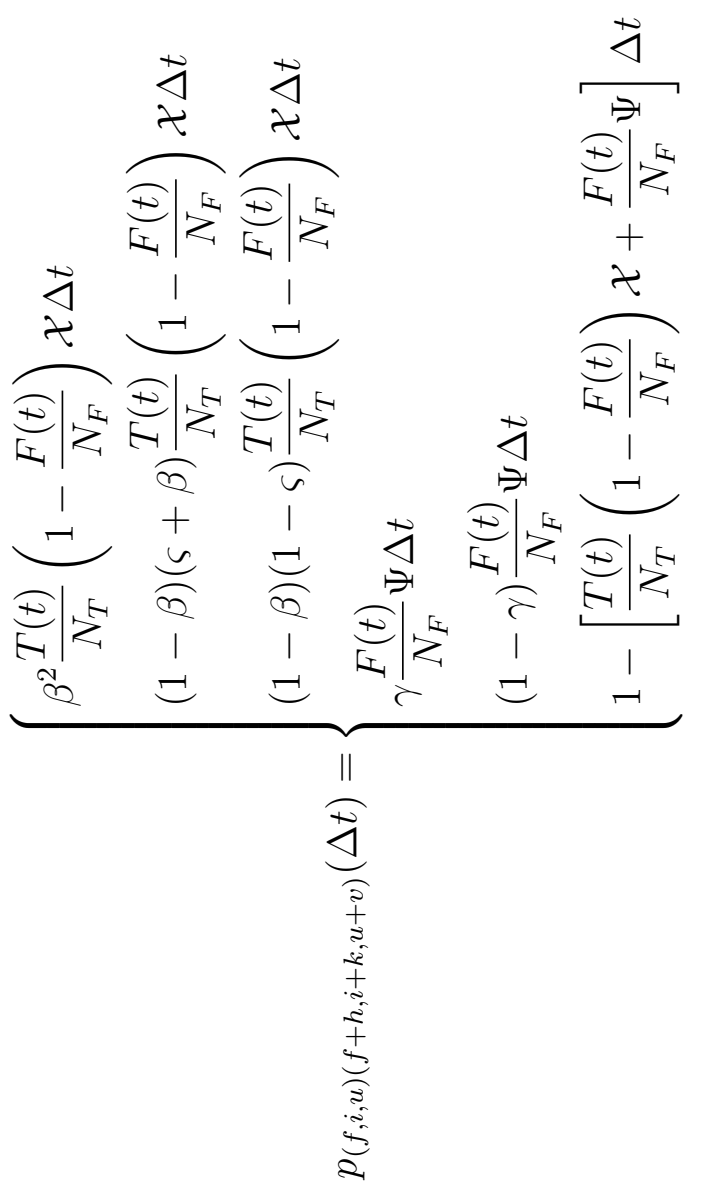




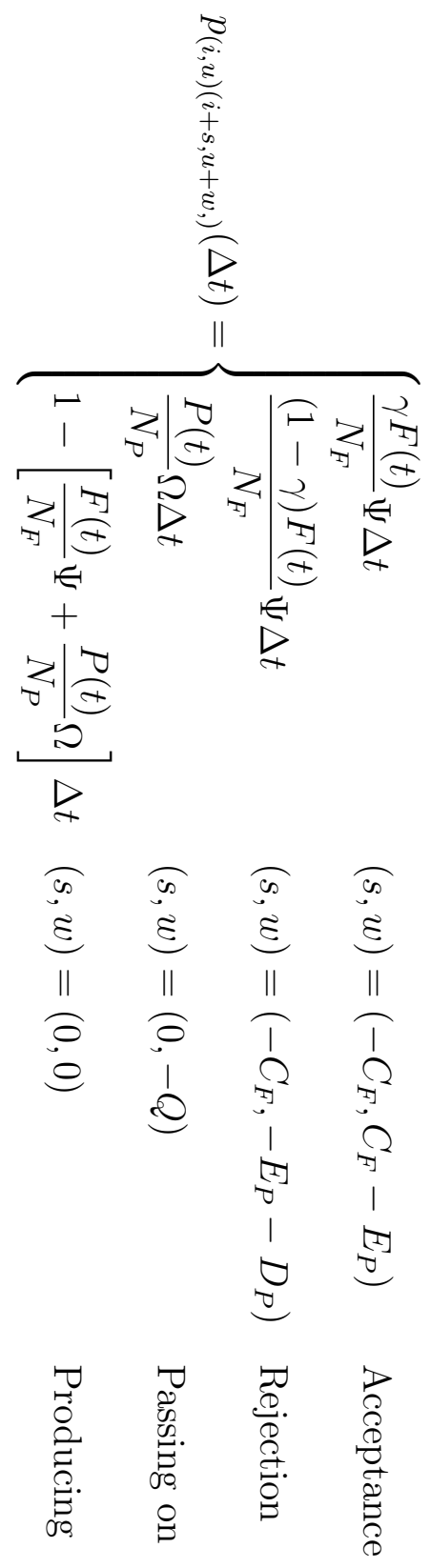


Because $T(t)$ and $F(t)$ change with time, we need to ensure Equation 2.14 holds for all values these can take.

There is no global maximum for the left hand side of Equation 2.14 but depending on the relative sizes of $\Phi$ and $\mathcal{X}$, we can estimate local maximums and then solve for the maximum allowable value of $\Delta t$. For $\Phi \geq \mathcal{X}$ the left hand side of Equation 2.14 is maximised when $T(t)=0$ giving

$$
\begin{array}{r}
{\left[(1-0) \Phi+0\left(1-\frac{F(t)}{N_{F}}\right) \mathcal{X}\right] \Delta t \leq 1} \\
\Longrightarrow \Phi \Delta t \leq 1 \\
\Longrightarrow \Delta t \leq \frac{1}{\Phi}
\end{array}
$$

If $\Phi<\mathcal{X}$, the left hand side of Equation 2.14 is maximised when $T(t)=N_{T}$ and $F(t)=0$

$$
\begin{array}{r}
{\left[\left(1-\frac{N_{T}}{N_{T}}\right) \Phi+\frac{N_{T}}{N_{T}}(1-0) \mathcal{X}\right] \Delta t \leq 1} \\
\Longrightarrow 0+\mathcal{X} \Delta t \leq 1 \\
\Longrightarrow \Delta t \leq \frac{1}{\mathcal{X}}
\end{array}
$$

Combining the two we can write

$$
\Delta t \leq \frac{1}{\max \{\Phi, \mathcal{X}\}}
$$

To ensure the transition probabilities in the factory reception stage always add to less than one we require

$$
\left[\frac{T(t)}{N_{T}}\left(1-\frac{F(t)}{N_{F}}\right) \mathcal{X}+\frac{F(t)}{N_{F}} \Psi\right] \Delta t<1
$$

In all of the scenarios presented in this thesis $\mathcal{X}>\Psi$, meaning the left side of the equation is maximised when $(T(t), F(t))=\left(N_{T}, 0\right)$. Thus the upper bound for $\Delta t$ in the factory reception stage is given by: 


$$
\begin{array}{r}
{\left[\frac{N_{T}}{N_{T}}\left(1-\frac{0}{N_{F}}\right) \mathcal{X}+\frac{0}{N_{F}} \Psi\right] \Delta t<1} \\
\frac{N_{T}}{N_{T}} \mathcal{X} \Delta t<1 \\
\Longrightarrow \Delta t<\frac{1}{\mathcal{X}}
\end{array}
$$

In the case of the processing stage, keeping the sum of the transition probabilities less than or equal to one requires

$$
\left[\frac{F(t)}{N_{F}} \Psi+\frac{P(t)}{N_{P}} \Omega\right] \Delta t<1
$$

This is maximised when $(F(t), P(t))=\left(N_{F}, N_{P}\right)$, giving

$$
\begin{aligned}
\left(\frac{N_{F}}{N_{F}} \Psi+\frac{N_{P}}{N_{P}} \Omega\right) \Delta t & <1 \\
(\Psi+\Omega) \Delta t & <1 \\
\Longrightarrow \Delta t & <\frac{1}{\Psi+\Omega}
\end{aligned}
$$

Combining the conclusions of Equations 2.15, 2.16, and 2.17 leads to the requirement

$$
\Delta t<\frac{1}{\max \{\Phi, \mathcal{X},(\Psi+\Omega)\}}
$$

\subsection{Multi-event Model Simulation Results and Discussion}

In this section we run simulations of the model developed in Section 2.6. We explore how the overall output is affected by testing at each stage, in order to identify the value potentially lost due rejection at each transition. This knowledge is useful for prioritisation of intervention and system improvement. 


\subsubsection{Parameter Values}

Some parameters, such as collection frequency, will remain the same throughout the following model simulations, regardless of scenario. Some of these parameters remain the same as they were in the previous model, some have changed slightly and some are new with this model. We will therefore discuss the estimates for these parameters before continuing with individual testing scenarios. We base the parameter values on data from Fonterra Co-operative Group, the dominant dairy company in the New Zealand dairy industry. As defined in Section 2.3.4, we are using a milk price of $\$ 4.40$. These parameters are all summarised in Table 2.7.2.

Milk Tanker Parameters The value collected from the farm vat, $V$, the frequency of collection, $\Phi$, and the frequency of delivery, $\mathcal{X}$, all remain the same as defined in Section 2.5.1.

$C_{T}$ is defined as the average capacity of one milk tanker.

As discussed in Section 2.5.1 each tanker has a capacity of about 28,800 litres $=2433 \mathrm{~kg}$ MS, therefore $C_{T}=\$ 10,700$.

$N_{T}$ is the capacity of the entire fleet of tankers. In this case, using a fleet of 525 tankers we have a capacity of $1,277,325 \mathrm{~kg} \mathrm{MS}$, which gives us $N_{T}=\$ 5,620,230$.

\section{Factory Reception Parameters}

$C_{F}$ Each processing site has multiple reception silos, as mentioned in Section 2.5.1, a typical paediatric site, producing infant formula, has silos of 225,000 litre capacity. This equates to 20,228 $\mathrm{kg} \mathrm{MS}$, giving $C_{F}=\$ 89,000$.

$N_{F}$ Given the typical paediatric processing site has three reception silos, with thirty three processing sites around the country this gives a total reception capacity of $N_{F}=\$ 8,811,000$.

$\Psi$ is the rate at which milk moves into the processing stage from the reception silos. In Section 2.5.1 we estimated a value of 311 movements per 
day, based on what would be necessary to keep up with production. In this section however, we add more flexibility to the model by estimating a realistic capacity for movement instead of just would would theoretically be necessary. Each separator is capable of separating 33,000 L of raw milk each hour. This gives $33 \times 3 \times 33,000 \times 24=78,408,000$ litres per day, which is equivalent to $6,923,463 \mathrm{~kg}$ MS. Dividing this by the capacity of one reception silo $C_{F}$ gives us $\Psi=343$.

\section{Initial Processing Parameters}

$N_{P}$ is the total capacity of the processing stage. As described in Section 2.5.1, the total capacity of separators and silos for separated product is $1,420,150$ litres which equates to $127,670 \mathrm{~kg} \mathrm{MS}=561,750$. As Fonterra has thirty three processing sites, we multiply this by thirty three, giving $N_{P}=\$ 18,537,750$.

$Q$ is the unit of milk leaving the processing stage each time step. We need to choose $Q$ small enough for the frequency of movement to be realistic but large enough that enough product can move for a given time step size. For our initial simulations we use $Q=\$ 187,000$.

$\Omega$ The rate which product leaves the processing stage and moves on to further processing remains as $\Omega=346$

\section{Time Step}

Using Equation 2.18 and substituting in the relevant parameter values, we can calculate the maximum allowable value for the time step as

$$
\begin{aligned}
\Delta t & \leq \frac{1}{\max \{11970,3990,(343+346)\}} \\
\Longrightarrow \Delta t & \leq \frac{1}{11970}=0.00008354 \text { days or } 7.218 \text { seconds }
\end{aligned}
$$

This suggests we use a time step size of seven seconds, giving $\Delta t=$ 0.00008102. This allows for an event to occur in almost every time step, while leaving some room for the stochastic nature of the model. 


\begin{tabular}{|c|l|r|}
\hline Parameter & Description & Initial values \\
\hline$V$ & Amount of milk collected from an on-farm vat & $\$ 3,050$ \\
\hline$\Phi$ & Frequency of collection a by tanker & 11,970 \\
\hline$E_{T}$ & Costs of testing milk at collection site & 0 \\
\hline$\alpha$ & Probability of acceptance by tanker & 3 \\
\hline $\mathcal{X}$ & Frequency of delivery to factory & 0 \\
\hline$E_{F}$ & Cost of testing milk upon delivery & 1 \\
\hline$\beta$ & Probability of passing tests upon arrival at factory & 0 \\
\hline$D_{F}$ & Cost of disposing of unwanted milk & 343 \\
\hline$\Psi$ & Frequency with which milk enters processing & 0 \\
\hline$E_{P}$ & Cost of testing prior to processing & 0 \\
\hline$\gamma$ & Probability of passing pre-processing tests & 346 \\
\hline$D_{P}$ & Cost of disposing of unwanted milk at factory level & $\$ 10,700$ \\
\hline$\Omega$ & Frequency of production & $\$ 5,620,230$ \\
\hline$C_{T}$ & Capacity of one Milk Tanker & $\$ 89,000$ \\
\hline$N_{T}$ & Capacity of tanker stage & $\$ 1,811,100$ \\
\hline$C_{F}$ & Capacity of one reception silo & $\$ 18,537,7000$ \\
\hline$N_{F}$ & Capacity of factory reception stage \\
\hline$Q$ & Process exit amount & \\
\hline$N_{P}$ & Capacity of Processing stage & \\
\hline
\end{tabular}

Table 2.5: Parameter values in the no risk scenario. All frequencies are the average number of occurrences per day.

\subsubsection{The Best of all Possible Worlds Scenario}

The initial set of simulations using the model outlined in Section 2.6 involve no testing cost or possible contamination, a scenario that would exist in a perfect world. The parameter values for this scenario are all as given in Table 2.5. We simulate this scenario, to check our model is producing as we might expect the supply chain to under these conditions. This scenario also gives us a good baseline to compare with simulation results from other scenarios.

A simulation of the total product produced over 24 hours is given in Figure 2.10, along with the deterministic solution discussed in Section 2.4.1. This particular simulation reaches a value of $\$ 26.5$ million worth of milk after 24 hours. Given that Fonterra can produce over $\$ 26$ million of product per day, this model reflects real world outcomes sufficiently. Over 500 simulations, each 24 hours in length, the average value of milk produced was $\$ 26.52$ million. The maximum produced in any of these simulations was $\$ 28.05$ million and the minimum $\$ 25.24$ million.

It is possible using this model to see the interaction between the states in Fig- 


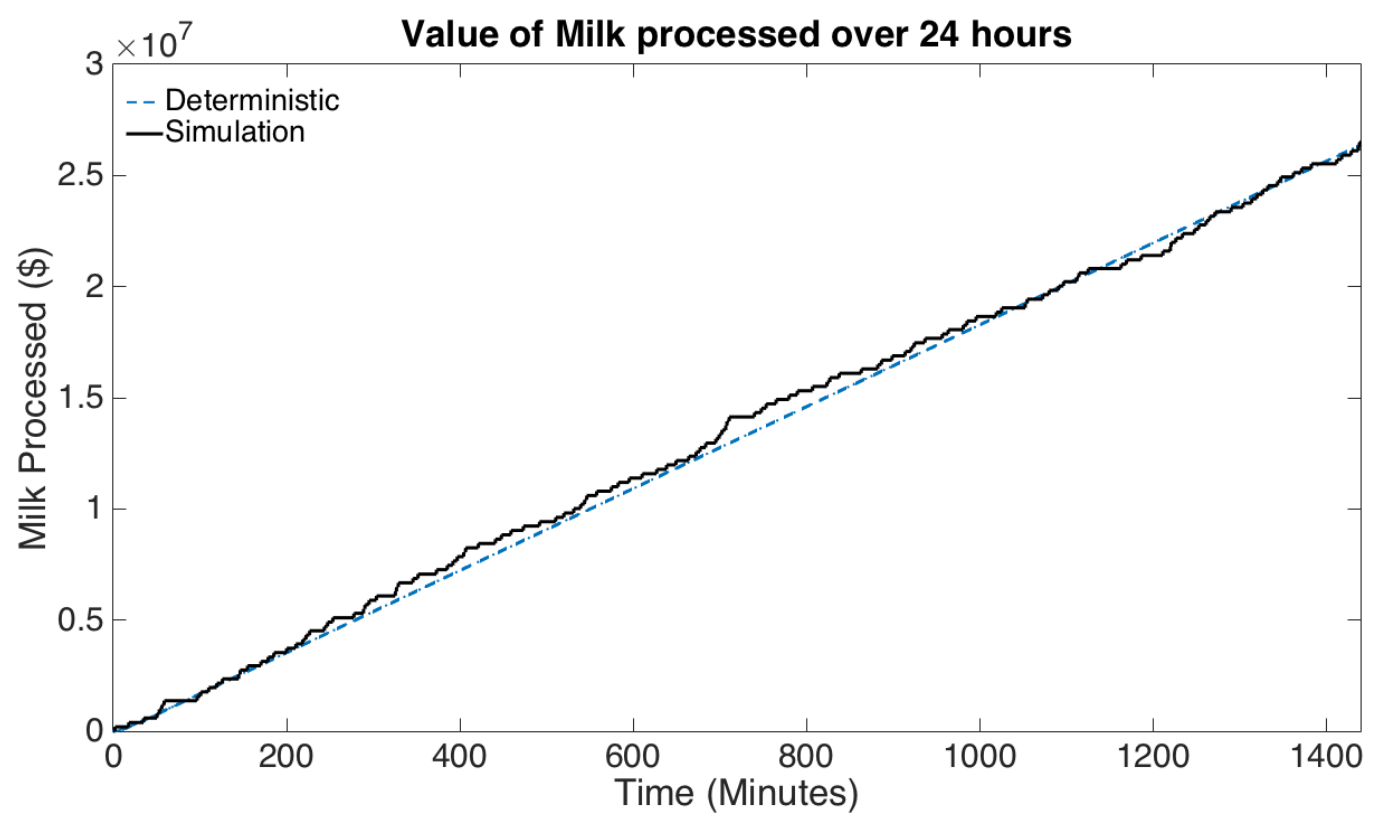

Figure 2.10: A simulation and the deterministic solution for the total value of the milk produced over a 24 hour period. The transition probabilities used are summarised in Section 2.6.4, and the parameter values are summarised in Table 2.5 .

ure 2.11. The changes are not large, due to the size restriction we have placed on the transitions, but a decreasing trend in the tanker stage is reflected by an increasing trend in the factory reception stage.

The dashed lines in Figure 2.11 show the deterministic solution for each stage. These are the values we would expect the simulations to average to given enough run time in equilibrium. We are using these equilibrium values as our initial values for these simulations, as we did. These values are given in Table 2.6 along with the average value after 500 simulations of 24 hours each.

\begin{tabular}{|l|r|r|r|r|r|}
\hline Stage & Initial Value & 500 Run Average & Minimum & Maximum & Std Dev \\
\hline Tanker & $\$ 1,540,200$ & $\$ 1,542,586$ & $\$ 7,1125$ & $\$ 2,089,800$ & $\$ 110,680$ \\
\hline Factory Reception & $\$ 3,824,300$ & $\$ 3,825,362$ & $\$ 992,620$ & $\$ 5,280,300$ & $\$ 306,480$ \\
\hline Processing & $\$ 184,990$ & $\$ 179,830$ & $\$ 99$ & $\$ 728,990$ & $\$ 77,447$ \\
\hline
\end{tabular}

Table 2.6: Simulation results for each production stage after 500 simulation runs in the perfect world scenario.

The processing stage shows the most variation. While this stage has the smallest standard deviation of the three stages, this is over half the average 


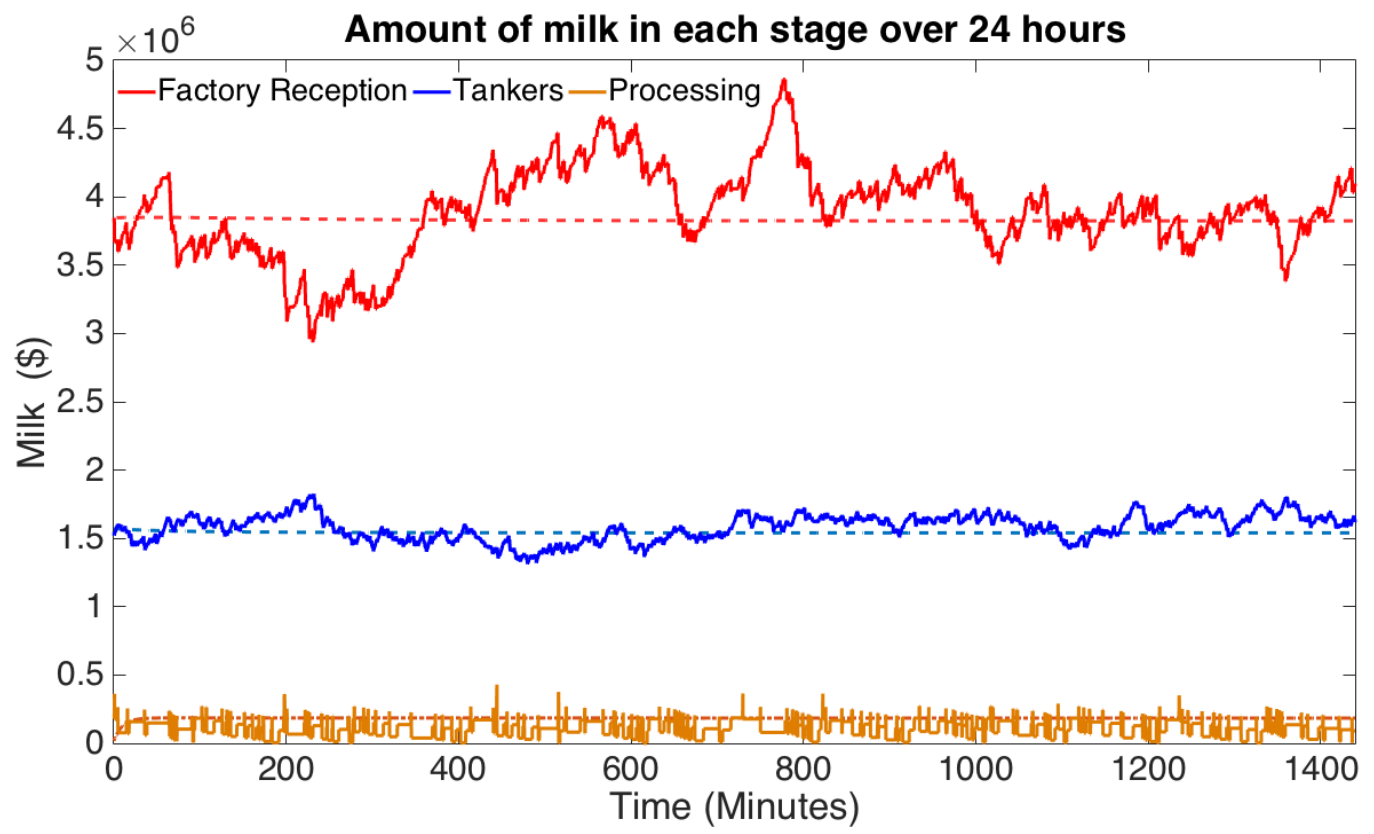

Figure 2.11: Plots of a simulation and the deterministic solution for the value of milk contained in each stage of the supply chain over 24 hours. The transition probabilities used are summarised in Section 2.6.4, and the parameter values are summarised in Table 2.5.

value of this stage. This is possibly due in part to the large amount of time the processing stage spends below $Q$, the minimum value required to pass product on. This may also explain the slightly larger difference in value we see between the stochastic simulation average and the deterministic equilibrium for this stage compared with the other two. Often, as soon as the processing stage reaches a value higher than $Q$, it will pass on product, dropping its value again.

\subsubsection{Testing Scenarios}

In this section we run simulations under various testing and rejection scenarios. Initially we run simulations using parameter values defined by real world information as discussed below.

\section{Initial Testing parameters}

$E_{T}$ is the estimated cost of testing milk when it is collected by the tanker. Based on information about testing in dairy mentioned in Section 2.3 
we will estimate $E_{T}=\$ 0.30$.

$\alpha$ is the probability that the milk passes all testing and is accepted by the tanker. Information obtained though discussion with Fonterra staff suggests we set $\alpha=0.9999$ (T. Kirk, oral communication, November 2015)

$E_{F}$ is the cost of testing milk as it arrives at the factory. Using test costs discussed in Section 2.3, we estimate $E_{F}=\$ 1.50$.

$\beta$ is the probability that a tanker load is accepted by factory. This is the stage with the greatest rate of rejection, with $1 \%$ of milk being discarded upon arrival at the factory (T. Kirk, oral communication, November 2015). Thus we set $\beta=0.99$.

$\varsigma$ is the probability the second tank of a tanker will be accepted, given that the first tank was rejected. Based on the number of farms visited by each tanker, there is about a 1 in 4 chance that the contaminated load spans both tanks. Taking this into account along with the possibility there is a second unrelated contamination we can estimate $\varsigma=0.75 \beta=0.7425$.

$D_{F}$ is the cost associated with disposing of milk when it is rejected at the factory reception stage. Depending on the reason for rejection, most rejected milk can be used as calf feed or sprayed on crops as fertiliser. Fonterra does contract tankers from outside their own fleet to transport this rejected milk, but the associated costs can generally recouped in the price paid for this rejected product. Because of this we set $D_{F}=0$.

$E_{P}$ is the cost of testing milk when it is passed on to the processing stage. Assuming the range of tests conducted pre-processing is similar to those conducted before acceptance into the factory, we set $E_{P}=\$ 1.50$.

$\gamma$ is the rate of rejection before entry into the processing stage. This stage has the lowest rejection rate of the three stages (T. Kirk, oral communication, November 2015). Once the milk is inside the factory the environment is much more controlled, the potential for contamination or spoilage is greatly reduced. We set the chance of rejection at $0.00001 \%$, implying $\gamma=0.99999$. 
$D_{P}$ is the cost associate with disposing of milk when it is rejected at the processing stage. As discussed in reference to $D_{F}$, disposal cost is negligible so we can set $D_{P}=0$.

These parameters are summarised in Table 2.7

\begin{tabular}{|c|l|r|}
\hline Parameter & Description & Initial values \\
\hline$V$ & Amount of milk collected from an on-farm vat & $\$ 3,050$ \\
\hline$\Phi$ & Frequency of collection a by tanker & 11970 \\
\hline$E_{T}$ & Costs of testing milk at collection site & $\$ 0.30$ \\
\hline$\alpha$ & Probability of acceptance by tanker & 0.9999 \\
\hline $\mathcal{X}$ & Frequency of delivery to factory & 3990 \\
\hline$E_{F}$ & Cost of testing milk upon delivery & $\$ 1.50$ \\
\hline$\beta$ & Probability of passing tests upon arrival at factory & 0.99 \\
\hline$\varsigma$ & Conditional 2nd acceptance probability & 0.7425 \\
\hline$D_{F}$ & Cost of disposing of unwanted milk & 0 \\
\hline$\Psi$ & Frequency with which milk enters processing & 343 \\
\hline$E_{P}$ & Cost of testing prior to processing & $\$ 1.50$ \\
\hline$\gamma$ & Probability of passing pre-processing tests & 0.99999 \\
\hline$D_{P}$ & Cost of disposing of unwanted milk at factory level & 346 \\
\hline$\Omega$ & Frequency of production & $\$ 10,700$ \\
\hline$C_{T}$ & Capacity of one Milk Tanker & $\$ 5,620.230$ \\
\hline$N_{T}$ & Capacity of tanker stage & $\$ 89,000$ \\
\hline$C_{F}$ & Capacity of one reception silo & $\$ 22,000$ \\
\hline$N_{F}$ & Capacity of factory reception stage & $\$ 18,537,700$ \\
\hline$Q$ & Process exit amount & \\
\hline$N_{P}$ & Capacity of Processing stage & \\
\hline
\end{tabular}

Table 2.7: Parameter values in the initial testing scenario. All frequencies are the average number of occurrences per day. Capacities and movement amounts are measured in NZ dollars worth of milk.

\section{Simulations for initial testing scenario}

The total value of milk produced over 24 hours, with 'everyday' acceptance parameters, is shown in Figure 2.12. The total output value reached after 24 hours is $\$ 26$ million. Over five hundred simulations this averages to $\$ 26.16$ million with a minimum of $\$ 24.68$ million and a maximum of $\$ 27.67$ million. This brings the average daily output value back close to Fonterra's actual daily production, compared with the optimistic value produced in the Section 2.7.2 simulations. 


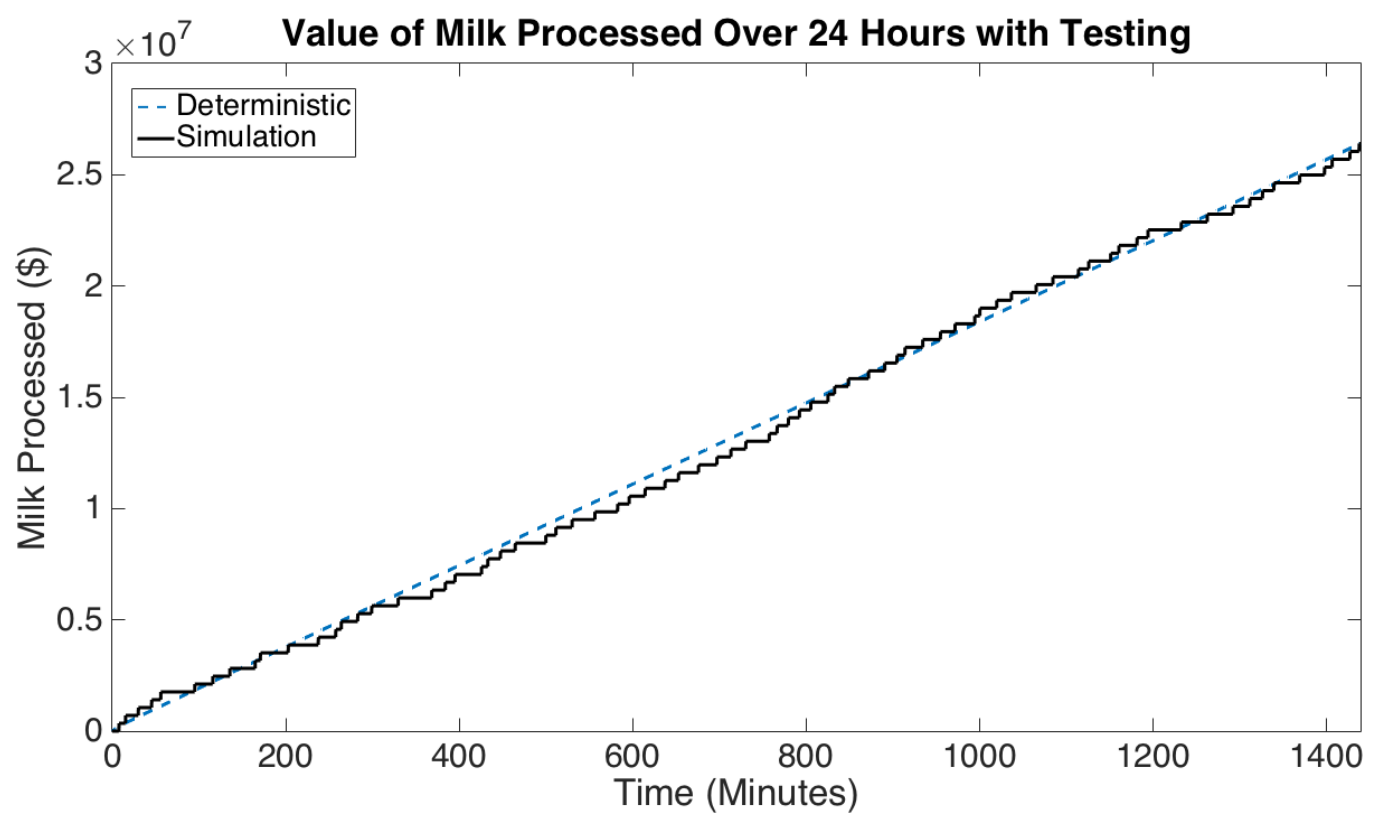

Figure 2.12: The total value of the milk produced over a 24 hour simulation period, along with the deterministic solution. The transition probabilities are those described earlier and summarised in Section 2.6.4. The parameters' values are summarised in Table 2.7.

The average values in each stage over time are given in Table 2.8. There is a slight drop in the level maintained by the tankers and factory receptions, compared with the previous simulation in Section 2.7.2. The most significant change however, is in the processing stage. The equilibrium value maintained in this stage has essentially been halved. Figure 2.13 plots a simulation for each of the three stages under this testing scenario.

\begin{tabular}{|l|r|r|r|r|r|}
\hline Stage & Initial Value & 500 Run Average & Minimum & Maximum & Std Dev \\
\hline Tanker & $\$ 1,532,700$ & $\$ 1,535,000$ & $\$ 1,019,000$ & $\$ 2,075,600$ & $\$ 110,680$ \\
\hline Factory Reception & $\$ 3,790,700$ & $\$ 3,790,800$ & $\$ 2,128,300$ & $\$ 5,175,200$ & $\$ 306,169$ \\
\hline Processing & $\$ 97,430$ & $\$ 99,100$ & $\$ 267$ & $\$ 551,280$ & $\$ 58,170$ \\
\hline
\end{tabular}

Table 2.8: Simulation results for the value contained in each production stage, averaged over five hundred runs using the initial testing scenario parameters as stated in Table 2.7.

\section{Simulations with various rejection probabilities}

We now investigate how changing the acceptance probabilities affects the overall output and the equilibrium values in each stage. We vary the accep- 


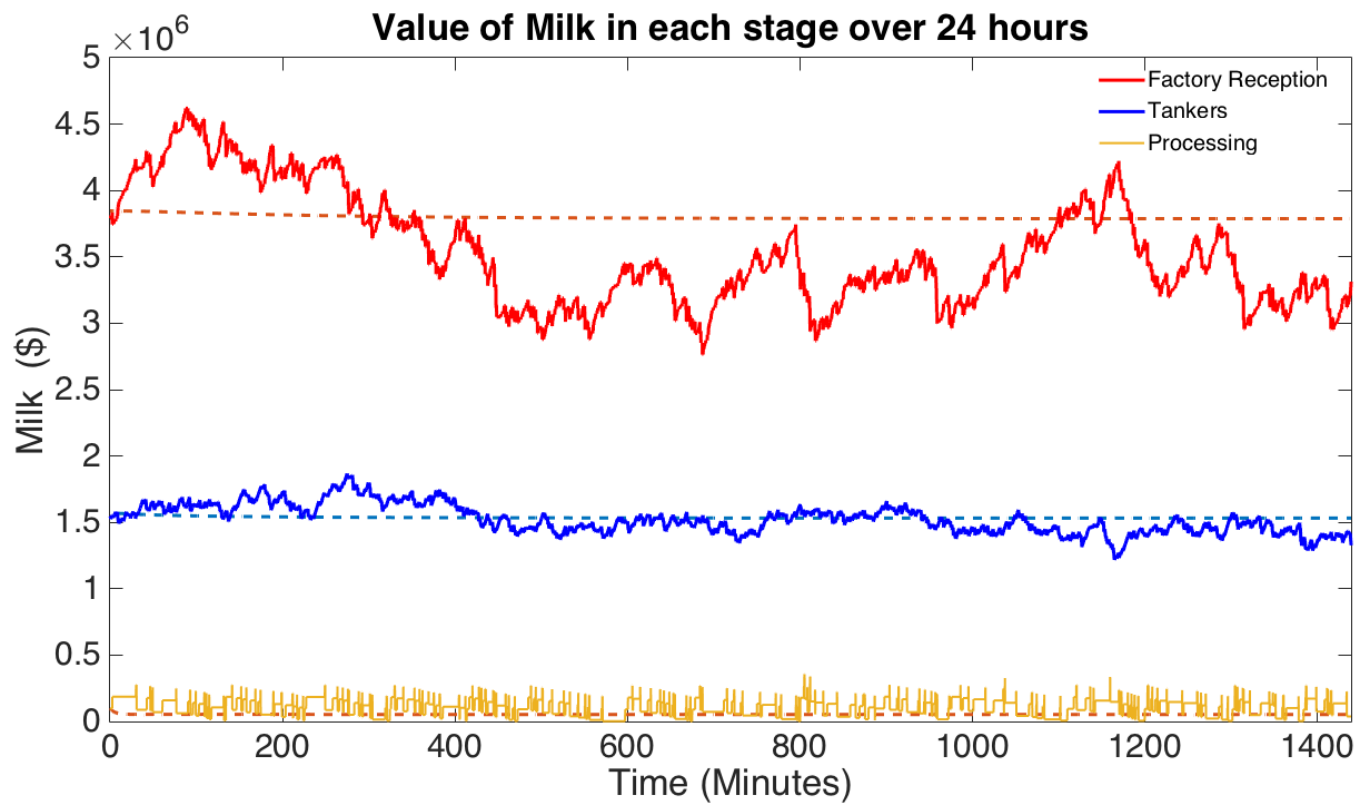

Figure 2.13: The value of milk in each stage, over 24 hours of simulation, and the deterministic solution. The parameters' values are summarised in Table 2.7.

tance probability of one stage while holding the probabilities in the remaining stages constant.

Milk Tanker acceptance: Figure 2.14 shows a simulation for the total output when we vary the probability of the milk tanker accepting milk presented for collection by farmers. We vary the value for $\alpha$ between 1 and 0.75 . Note that there is a lot of variability in a single simulation and, as seen in Figure 2.14, at certain times the amount of milk processed may be the same for multiple values of $\alpha$. Table 2.9 gives the five hundred simulation average for each of these acceptance values. The output in this case varies between $\$ 26.5$ million, in a good run with acceptance probability $\alpha=1$, and $\$ 22.2$ million for a run where $\alpha=0.75$. This a potential difference of $\$ 4.3$ million over one day of production.

Factory Acceptance Now we will vary the acceptance rate upon arrival at the factory reception, again keeping the other variables are helt constant at the values given in Table 2.7. Simulations with various probabilities of accepting milk as it enters the factory reception stage are shown in Figure 2.15. As noted for Figure 2.14, due to the variability in a single simulation, 


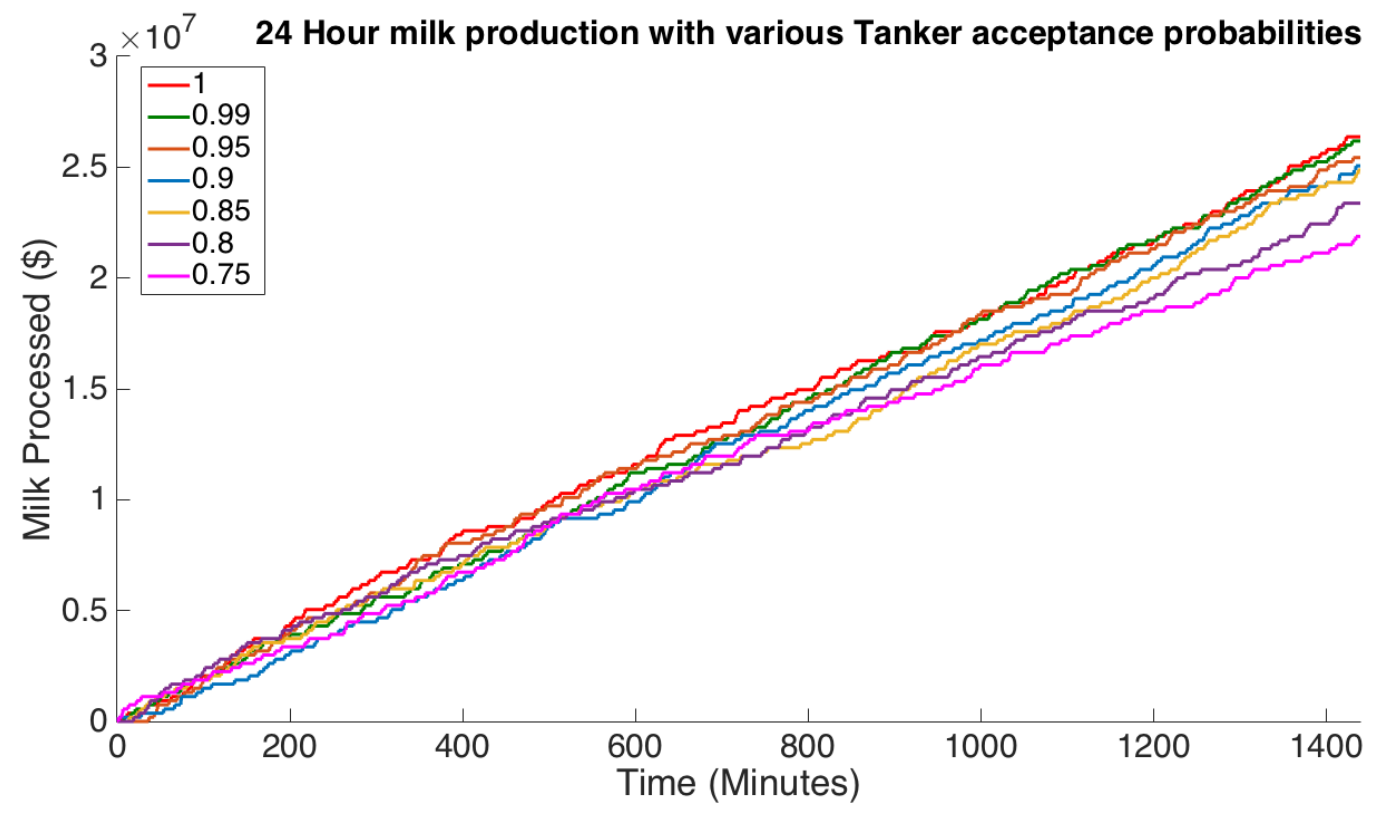

Figure 2.14: The total value of the milk produced over a 24 hour period for seven different vat acceptance probabilities. All other parameters remain constant at the values given in Table 2.7

\begin{tabular}{|c|c|c|c|}
\hline $\begin{array}{c}\text { Tanker Acceptance } \\
\text { Probability }(\alpha)\end{array}$ & $\begin{array}{c}\text { 500 Run } \\
\text { Average }\end{array}$ & $\begin{array}{c}\text { Lower } \\
\text { Quartile }\end{array}$ & $\begin{array}{c}\text { Upper } \\
\text { Quartile }\end{array}$ \\
\hline 1 & $\$ 26,167,284$ & $\$ 25,806,000$ & $\$ 26,554,000$ \\
\hline 0.99 & $\$ 26,050,970$ & $\$ 25,619,000$ & $\$ 26,367,000$ \\
\hline 0.95 & $\$ 25,553,176$ & $\$ 25,245,000$ & $\$ 25,993,000$ \\
\hline 0.9 & $\$ 24,811,160$ & $\$ 24,497,000$ & $\$ 25,245,000$ \\
\hline 0.85 & $\$ 24,112,154$ & $\$ 23,849,000$ & $\$ 24,310,000$ \\
\hline 0.8 & $\$ 23,306,932$ & $\$ 23,001,000$ & $\$ 23,375,000$ \\
\hline 0.75 & $\$ 22,515,922$ & $\$ 22,253,000$ & $\$ 22,814,000$ \\
\hline
\end{tabular}

Table 2.9: Simulation results for the average daily production over 500 simulations for various milk tanker acceptance probabilities. Factory reception and processing acceptance probabilities remain constant.

at certain times the amount of milk processed may be the same for multiple values of $\beta$. Comparing these two plots visually however, Figure 2.15 does show more vertical spread, each line is more distinct from the others in the plot, when compared to those in Figure 2.14.

As highlighted in Table 2.10 the value of milk produced in a 24 hour period varies between $\$ 26.7$ million worth of milk for a high simulation with $100 \%$ acceptance of tanker deliveries, and $\$ 19.8$ million for a low producing simu- 


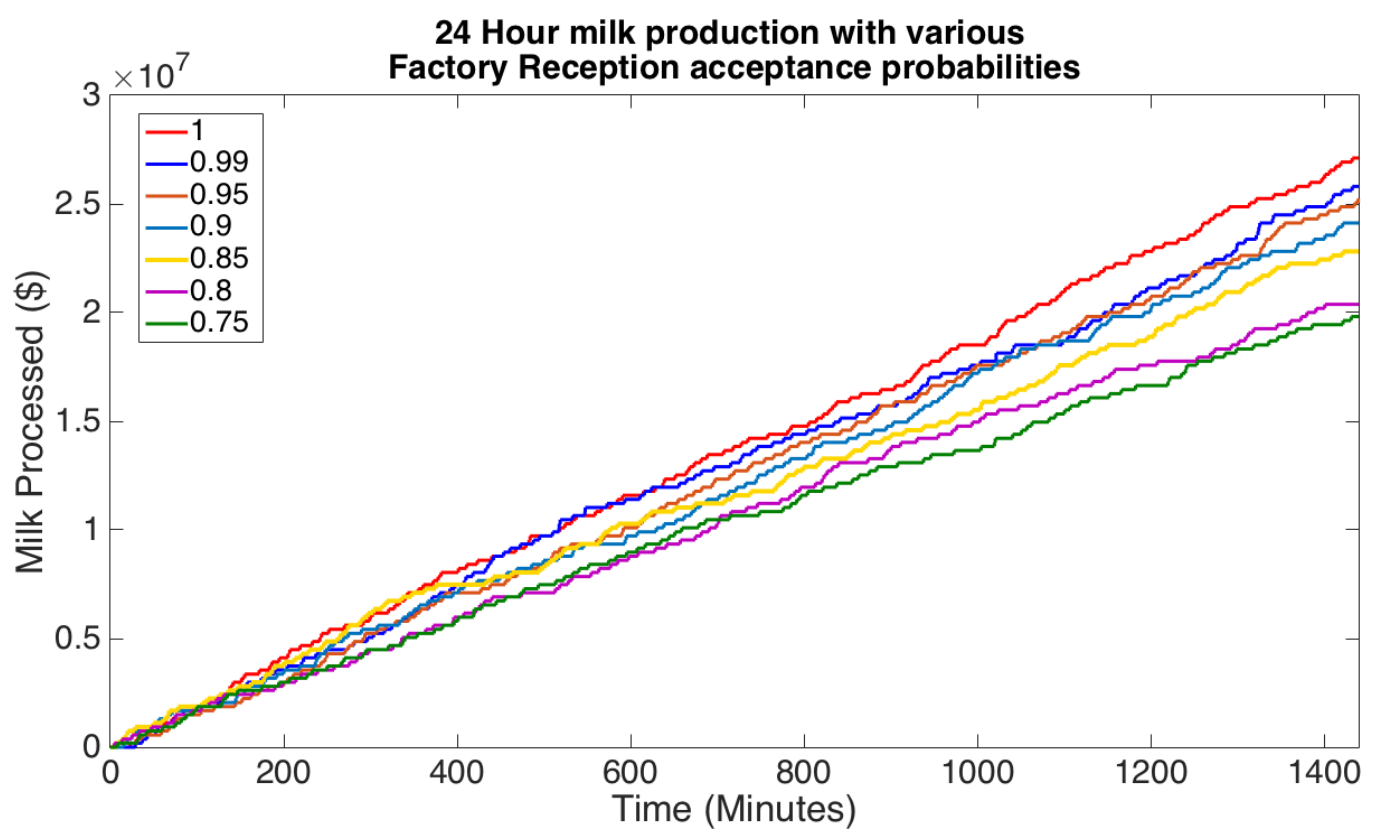

Figure 2.15: The total value of the milk produced over a 24 hour period for seven different milk factory reception acceptance probabilities. All other parameters remain constant at the values in Table 2.7.

lation with only $75 \%$ acceptance. That is a total potential difference of $\$ 6.9$ million, in just one day of production. Compared with the variation we saw when varying the acceptance rate of tanker collections in Table 2.9, rejection of product at this stage shows greater effect on the overall value produced, implying greater potential loss for similar rejection rates.

\begin{tabular}{|c|c|c|c|}
\hline $\begin{array}{c}\text { Factory Acceptance } \\
\text { Probability }(\beta)\end{array}$ & $\begin{array}{c}\text { 500 Run } \\
\text { Average }\end{array}$ & $\begin{array}{c}\text { Lower } \\
\text { Quartile }\end{array}$ & $\begin{array}{c}\text { Upper } \\
\text { Quartile }\end{array}$ \\
\hline 1 & $\$ 26,413,657$ & $\$ 25,993,000$ & $\$ 26,741,000$ \\
\hline 0.99 & $\$ 26,181,496$ & $\$ 25,806,000$ & $\$ 26,180,000$ \\
\hline 0.95 & $\$ 25,177,680$ & $\$ 24,824,000$ & $\$ 25,619,000$ \\
\hline 0.9 & $\$ 23,933,756$ & $\$ 23,562,000$ & $\$ 24,310,000$ \\
\hline 0.85 & $\$ 22,592,592$ & $\$ 22,253,000$ & $\$ 23,001,000$ \\
\hline 0.8 & $\$ 21,352,034$ & $\$ 20,944,000$ & $\$ 21,692,000$ \\
\hline 0.75 & $\$ 20,144,014$ & $\$ 19,822,000$ & $\$ 20,383,000$ \\
\hline
\end{tabular}

Table 2.10: Simulation results for the average daily production over 500 simulations. Values are given for simulations using various values for $\beta$ between 0.75 and 1 , while holding all other parameters constant. 
Processing Acceptance Now keeping all other parameters constant, as presented in Table 2.7, we vary the rate of acceptance at the processing stage. Simulations for various probabilities of accepting milk for processing, using values from $\gamma=0.75$ to $\gamma=1$, are shown in Figure 2.16.

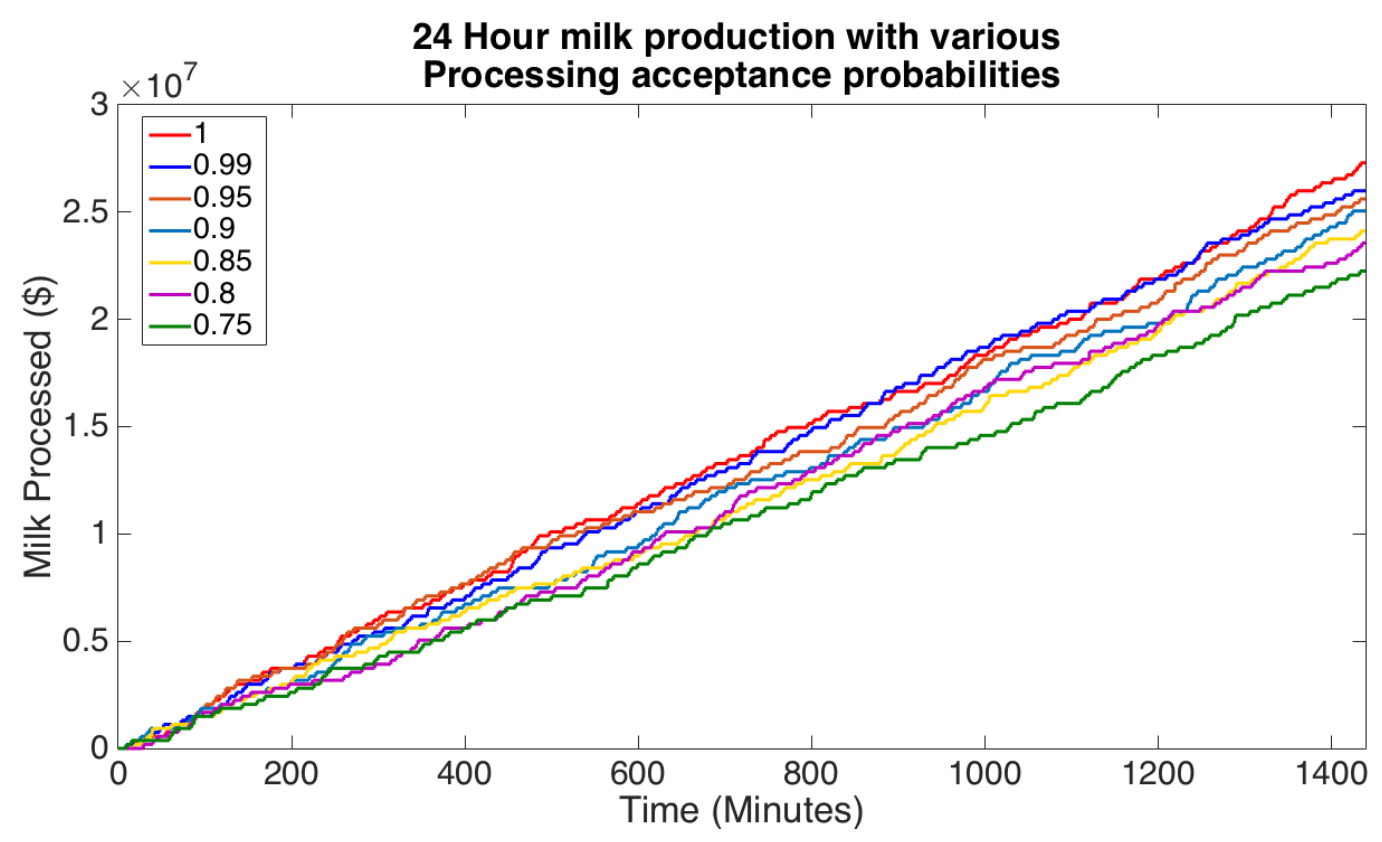

Figure 2.16: The total value of the milk produced over a 24 hour period for seven different milk tanker acceptance probabilities. The value of $\gamma$ is varied between 0.75 and 1 , while $\alpha$ and $\beta$ remain the same as in Table 2.7, along with the rest of the parameters. The step size is $\Delta t=8$ Seconds.

Visually this plot does not show as much vertical spread as Figure 2.15, the plot of various factory reception acceptance rates. Comparing this plot with tankers' various rates of rejecting farm vats, as shown in Figure 2.14, a difference in spread is not as discernable.

Table 2.11 Shows the average 24 hour output for various values of $\gamma$ after five hundred simulations. Production value in this case varies between $\$ 26.5$ million for a good run with $\gamma=1$ and $\$ 21$ million for a simulation with $\gamma=0.75$. This is a potential difference of $\$ 5.5$ million over one day of production. This implies a spread closer to that of varying acceptance rates at entry to the factory reception than to what is seen when we cary the rate at which tankers accept farm vats. 


\begin{tabular}{|c|c|c|c|}
\hline $\begin{array}{c}\text { Processing Acceptance } \\
\text { Probability }(\gamma)\end{array}$ & $\begin{array}{c}\text { 500 Run } \\
\text { Average }\end{array}$ & $\begin{array}{c}\text { Lower } \\
\text { Quartile }\end{array}$ & $\begin{array}{c}\text { Upper } \\
\text { Quartile }\end{array}$ \\
\hline 1 & $\$ 26,205,806$ & $\$ 25,993,000$ & $\$ 26,554,000$ \\
\hline 0.99 & $\$ 26,050,596$ & $\$ 25,619,000$ & $\$ 26,367,000$ \\
\hline 0.95 & $\$ 25,307,084$ & $\$ 24,871,000$ & $\$ 25,806,000$ \\
\hline 0.9 & $\$ 24,470,820$ & $\$ 24,123,000$ & $\$ 24,871,000$ \\
\hline 0.85 & $\$ 23,463,638$ & $\$ 23,001,000$ & $\$ 23,936,000$ \\
\hline 0.8 & $\$ 22,578,380$ & $\$ 22,253,000$ & $\$ 23,188,000$ \\
\hline 0.75 & $\$ 21,516,968$ & $\$ 21,084,000$ & $\$ 22,066,000$ \\
\hline
\end{tabular}

Table 2.11: Simulation results for various values of $\gamma$. The average over 500 simulation runs for selected values of $\gamma$ between 0.75 and 1 .

\subsubsection{Discussion}

One of the main aims of this model and simulations is to see at which stage rejection of product has the most impact. Table 2.12 shows the average 24 hour production outcomes for the scenarios simulated in Section 2.7.3. For each column only the stated acceptance parameter is changed while the other two remain constant at the values presented in Table 2.7.

\begin{tabular}{|c|c|c|c|}
\hline Acceptance & \multicolumn{3}{|c|}{ Parameter Varied } \\
\cline { 2 - 4 } Z Probability & $\alpha$ & $\beta$ & $\gamma$ \\
\hline 1 & $\$ 26,167,284$ & $\$ 26,413,657$ & $\$ 26,205,806$ \\
\hline 0.99 & $\$ 26,050,970$ & $\$ 26,181,496$ & $\$ 26,050,596$ \\
\hline 0.95 & $\$ 25,553,176$ & $\$ 25,177,680$ & $\$ 25,307,084$ \\
\hline 0.9 & $\$ 24,811,160$ & $\$ 23,933,756$ & $\$ 24,470,820$ \\
\hline 0.85 & $\$ 24,112,154$ & $\$ 22,592,592$ & $\$ 23,463,638$ \\
\hline 0.8 & $\$ 23,306,932$ & $\$ 21,352,034$ & $\$ 22,578,380$ \\
\hline 0.75 & $\$ 22,515,922$ & $\$ 20,144,014$ & $\$ 21,516,968$ \\
\hline
\end{tabular}

Table 2.12: Simulation results for the average daily production over five hundred simulations. Values are given for simulations using various acceptance probabilities for each stage, while keeping all other parameters the same as in Table 2.7

As was mentioned in Section 2.7.3, variation in the acceptance probability of milk being delivered to the factory reception stage results in the greatest variation in total output value. This suggests that the entry to the factory reception is a point in the supply chain that should be prioritised in terms of increasing acceptance and probability minimising product loss. 


\section{Suggestions for ixncreasing acceptance rates}

One possible method for increasing acceptance rates at the factory reception could be to improve the accuracy of testing when the tanker collects milk from the farm. If we were able to remove all contamination before the milk even enters the tanker then we could theoretically reduce the need to reject material later. Looking at Table 2.12, reducing the probability that the milk tanker accepts milk from a farm has less of an impact than reducing the probability of acceptance at either of the other two stages. This suggests if we were able to increase the acceptance of tankers delivering milk to the factory by being more strict with the milk that is accepted from farms, and potentially seeing more product rejected by tankers, we may see greater output overall. There are obviously costs and other issues involved in implementing this proposition such as appropriate tests not being available at this point, or analysis taking too long to wait for, but it warrants further investigation. If the necessary testing could be implemented at a cost less than the increase in output it would be a worthwhile investment.

Another possible solution could be investing in refrigerated milk tankers. In reality Fonterra's milk tankers are currently unrefrigerated, so accidents and unforeseen delays can result in good milk being spoilt during transport. This would essentially increase $\beta$, the probability of accepting milk when it it is first delivered to the factory by the tanker. We do not have access to data on how often this occurs, but if the loss reduction is large enough this could be a good option. Especially given that we have identified the loss of milk as it is delivered to the factory as having the biggest impact on overall output of the three stages modelled.

A third solution is to improve our knowledge of where contaminated milk has come from and exactly which products may be influenced. Essentially this is what we would expect to achieve through a good traceability system. This would allow us to only discard the substandard product and retain as much unaffected product as possible for further production stages. This may or may not include extra testing costs along with the costs of tracking the product though the supply chain, but could effectively reduce rejection rates without requiring more intensive testing regimes as might be required in the first option. 
All three of these suggestions could be valid methods of reducing product rejection rates and increasing overall output. Further investigation of intensifying testing at one or two stages to reduce rejection rates at others could easily be done using the model described in this chapter. The effect of reducing losses by knowing more about our supply chain would require a little more work but again this model provides a good starting point. Investigation of refrigerated tankers is plausible but obtaining appropriate data may be a challenge.

\subsection{Conclusions}

We have developed a working model for the flow of milk from the farm through the first stage of processing. This model allows for testing costs and resulting rejection of substandard product. Through various simulations we have seen the effect of different rejection rates at each stage of production. Other than including testing costs or not, we have not shown how the cost parameters impact the total output value. In applying the model to Fonterra any realistic costs did not show much impact on overall output, likely due to their small size relative to Fonterra's daily output value. This may be an area that requires more investigation however, particularly in the case of smaller companies where such costs may be more significant.

The results of our simulations suggest the probability of accepting tanker deliveries to factory reception has the biggest impact on overall production compared with the other two possible rejection points in our model. This result suggests further investigation into managing product rejection and in particular reducing tanker delivery rejection if possible. Some possible solutions for managing product loss are discussed, but further investigation is needed in this area.

The model developed here forms a good basis into which other aspects of production may be incorporated to investigate their potential impacts on overall dairy production. For example, by manipulating capacities the effects of various batch sizes on overall output under various conditions could be analysed. 


\section{Chapter 3}

\section{Modelling New Zealand Dairy Production: The Impact of Traceability from the Farm to the Factory}

\subsection{Introduction}

Traceability has become an increasingly important research area in recent years. It has always been an important aspect of production, but recent contamination events have highlighted its significance. Increasing complexity in widespread international supply chains, along with varying standards across countries, means traceability has become even more important in maintaining international reputations and trade. Ideally food safety standards would be such that contaminations did not occur at all, but this is just not possible in reality. Traceability is required to manage defects and contaminations that do occur, reducing their impact, both economically and in regards to public health.

Milk and dairy products from domestic animals have been used by people for hundreds of years (Chand, 2006). They have long been an important part of the western style diet and are increasingly being consumed as part of Asian diets as well (Tsuda et al., 2000). Dairy products are a valuable source 
of essential nutrients, and are often a large component of young children's diets. The potential risk to young children means milk and its derivatives are particularly vulnerable to contamination scares. The 2008 melamine contamination of infant formula in China, the Fonterra botulism scare in 2013, and the 2015 poisoning threat to baby formula in New Zealand are all examples of this.

The 2013 Botulism scare in particular highlights the need for efficient, accurate, testing and traceability. The amount of time taken in the scare of 2013 to confirm the sources of the contamination, and the location of the contaminated batches, risked not only Fonterra's reputation but New Zealand's reputation as an exporter. New Zealand is a world leader when it comes to the production and export of dairy products (Jiang \& Sharp, 2014). The dairy industry forms a large part of the country's exports. Both of these aspects make the New Zealand dairy industry a good case to apply and develop our model.

This chapter builds on a Markov chain model developed in Chapter 2. We incorporate traceability parameters and effects into this model in order to investigate the potential value a good traceability system can contribute to the initial stages of the dairy supply chain.

Following this introduction, Section 3.2 gives an overview of the dairy industry, with a brief look at the history of dairy production and product importance. The second half of the section covers aspects of specific importance to the New Zealand dairy industry. We follow this with a review of traceability in Section 3.3. We cover the definition of traceability, why it is important and the approaches taken by previous literature. In Section 3.4 we discuss the use of discrete time Markov chains (DTMCs) to model the flow of value through the dairy supply chain. We introduce the stages of the model and develop the transition probabilities to include the effects of a traceability system. Finally, we present simulation results and discuss implications of this work. 


\subsection{Dairy}

Dairy products have been an important part of the western style diet for centuries. Milk is rich in a variety of essential nutrients (Steijns, 2001), and the worldwide market for dairy and milk based products continues to grow (Huffman \& Harper, 1999). Along with this growth comes increasing food safety issues, with consumer perception becoming increasingly important (Etzel et al., 2012). Aside from its value as milk, many derived dairy products are available. In particular, functional foods and health supplements made with milk proteins have proven to be of considerable value (Tomé \& Debabbi, 1998). Steijns (2001) discusses various components of dairy products and their role in managing a variety of health concerns. Research has also been done into how certain dairy products may be useful for cancer prevention (Tsuda et al., 2000; Parodi, 2001).

As mentioned earlier, New Zealand is a world leader in the production and export of dairy products (Jiang \& Sharp, 2014). The dairy industry in this country is mainly pasture based (Chand, 2006). The New Zealand dairy industry has earned a reputation for its low cost, high quality systems and technological expertise. About $97 \%$ of New Zealand dairy farmers sell their milk through Fonterra Cooperative Group (Jiang \& Sharp, 2014). Cows are generally milked twice per day (Hogeveen \& Ouweltjes, 2002), and milk is collected from the farm in a tanker every one to two days (Jiang \& Sharp, 2014). Fonterra operates a national fleet of 525 tankers collecting from around eleven thousand farms (Mason, 2014). The frequency of collection is generally dependent on the time of year, as milk production is seasonal. The amount of milk a farmer is allowed to supply to Fonterra is limited by the number of shares they own in the cooperative. Because of this, output becomes targeted. The cost efficiency of New Zealand dairy farms is examined by Jiang \& Sharp (2014), whose results indicated that there is still room for improvement. Trends in developing high capacity milking parlours and automatic miking systems have seen an increase in cow throughput, along with reduced manual labour on dairy farms. As these trends continue, further labour based barriers to farm expansion may be overcome (Hogeveen \& Ouweltjes, 2002).

Because dairy products are such a large part of so many diets, and especially for young children, they are particularly vulnerable to contamination scares. 
While reliable testing and quality standards are important for all consumable products, they are particularly important for dairy. The Fonterra supplier's handbook lists at least nine contamination types to be tested for along with general quality grading and organoleptic assessment, though only two of these tests are conducted upon tanker collection every time (Fonterra Co-operative Group, 2014b). While New Zealand is known for low cost dairy production, research by Jiang \& Sharp (2014) indicates that there is still room for improvement. Traceability can be incorporated into testing and quality control systems to increase certainty of safe product, as well as potentially improve efficiency in the production chain.

\subsection{Traceability}

Traceability is the capability to trace goods throughout the distribution chain (Tamayo et al., 2009). It is a widely used concept, with various approaches studied over the last few decades. The study and implementation of traceability requires an interdisciplinary approach, spanning the natural and social sciences(Karlsen et al., 2013). The aim of a traceability system is to collect information relevant to the location of products along the supply chain (Dabbene \& Gay, 2011), allowing the flow of material to be followed (Karlsen et al., 2013).

In the event of a product contamination or other fault, traceability becomes very important. Having an appropriate traceability system and an efficient recall plan in place is critical for the proper management of supply chain risks, particularly in today's global market (Kumar \& Schmitz, 2011). Traceability makes selective recalls possible (Tamayo et al., 2009), with no traceability it is difficult to determine how far a contamination has spread, necessitating a widespread recall and the very real possibility of contaminated product being consumed by the public. To be effective in a recall situation, a traceability system must be able to trace back along the supply chain to the source of the contamination as well as forward to identify all the affected product (JansenVullers et al., 2003). An effective traceability system allows the efficient and precise withdrawal of contaminated product. Such efficient product withdrawal mitigates costs associated with a contamination scare, and reduces 
the potential risk to consumers health (Resende-Filho \& Buhr, 2010). The precision of a traceability system is also important. This is best understood as a ratio between the size of identifiable units at two points in the supply chain (Bollen et al., 2007) and can determine how much product is recalled and the value lost (Dabbene \& Gay, 2011), as well as the time and effort required to locate all of the faulty product. Efficient location allows for reduced spread and therefore a reduced impact on consumer confidence. Good traceability is not about reducing the probability of a contamination event, but about reducing the consequences if contamination does occur (Dupuy et al., 2005). Buhr (2003) identifies traceability as crucial to a firm's ability to limit the size and spread of a recall.

Estimating the value of a traceability system can be difficult, as the return is essentially the loss avoided in the case of a contamination or other production fault (Dupuy et al., 2005). Resende-Filho \& Buhr (2010) develop conceptual and process simulation models to investigate the value of traceability for food recalls. Incorporating quality control, they identify key factors affecting the value of a traceability system. Their focus is on the economic modelling of traceability as a tool to reduce the extent and size of a recall. A case study of E. coli in ground beef is presented. It is suggested that the main value of a traceability system lies in its ability to improve the recall process though records management and verification.

While traceability is important for food safety and reducing the potential impact of contamination events, it can also be used to optimise production planning and scheduling, creating a competitive advantage. X. Wang \& Li (2006) propose frameworks to achieve business benefits through the integration of traceability and supply chain management processes. They provide a case study of a British meat processing company as illustration. Canavari et al. (2010) discuss traceability as part of information management in supply chains, they suggest traceability can be an important part of a firms competitive strategy.

Within the American private sector there has been widespread voluntary adoption of food traceability systems in order to improve efficiency in the event of a recall, particularly in the grain sector where supply management and demand for high-value attributes lead firms to differentiate and track production (Golan et al., 2004). The food industry as a whole has also responded 
to food safety crises by implementing quality assurance and traceability systems of their own, beyond what is legislated (Trienekens \& Zuurbier, 2008). Firms' reputations are an important consideration when providing incentives for them to deliver safe high-quality goods (Carriquiry \& Babcock, 2007). Good traceability reduces anonymity in the supply chain, hence it can also be useful in identifying who may be liable in the event of a contamination or fault (Resende-Filho \& Buhr, 2010). This also provides an incentive for firms to improve their safety and quality standards. As the probability that they will be held accountable increases, firms seek to improve their own standards (Pouliot \& Sumner, 2008). American firms interviewed by Resende-Filho \& Buhr (2010) did not know the cost of a recall, but did view the resulting loss of product sales as the primary cost.

Various approaches to the problem of modelling traceability and its effects in a supply chain are outlined in literature. These mainly include optimisation using mathematical programming or systems of differential equations. Dupuy et al. (2005) propose a mathematical mixed integer linear programming (MILP) model for a batch dispersion problem. They aim to optimise the traceability system such that the impact of a recall is reduced. Their model is applied to a sausage manufacturing process in a French food company. Their results confirm the idea that smaller batch sizes result in lower recall costs. While they conclude that their model is too large for daily industry use, they suggest their method could be applied to simpler models. Dabbene \& Gay (2011) build on the work done by Dupuy et al. (2005). They introduce a modelling framework and optimisation strategy and use recall cost to measure and optimise the performance of traceability systems. As in Dupuy et al. (2005), they express the optimisation problem in the form of a MILP model. They model the flow of product batches through the supply chain via a directed graph. The nodes of this graph represent product processing-units or storage containers in the supply chain. The capacity of a given node is bounded by the amount of product that can be processed or stored by the infrastructure at that node at one time. An improvement made by this model is the ability to account for the quantities being moved, not just where and when. They describe an approach to account for either the worst-case recall cost, or the average recall cost. Numerical examples are provided based on the same sausage scenario presented in Dupuy et al. 
(2005).

Most current methods for precisely estimating the amount of product that needs to be discarded in a recall event are only available for products that exist in discrete units and are made using discrete units of ingredients (Dabbene \& Gay, 2011). Traceability becomes increasingly difficult when fluid ingredients, such as liquids, powders, or even grains, are involved in the supply chain (Comba et al., 2013). Bollen et al. (2007) considers the case of apples being processed in a pack house. The apples are supplied in large bulk bins and transferred via a water dump, mimicking a fluid product, to a grader where they are sorted and directed into packaging lines. Using experiments running blue marker balls through the processing system, they collect data on apple arrival times. This data is then used to develop a stochastic model that can estimate the probability that an individual apple came from a particular bin, based on its arrival position. While apples are discrete items, they flow though this production chain in a very fluid manner, with similar opportunities for mixing to that of small particles. Skoglund \& Dejmek (2007) use dynamic simulation models to analyse the change over of liquid lots in a pipe. The partial mixing that occurs in sequential lots leads them to introduce the concept of fuzzy traceability. Comba et al. (2013) present an approach to manage traceability of bulk products during production, storage, and delivery. They present a model that combines differential equations with discrete events, based on the asynchronous opening and closing of valves and activation of pumps that control the flow of product. Their approach makes it possible to monitor the mixing of materials, providing the basis for an efficient recall process in a system that involves fluid components.

The literature outlined above is largely deterministic. This chapter fills a gap in traceability literature by considering a stochastic model using Markov chains. It also adds to the limited literature on modelling fluid product supply chains. A model for the flow of milk from the farm to the factory is developed in Chapter 2. Markov chains are used to simulate dairy production under different testing conditions and with various rates of product rejection. The model in this chapter builds on that developed in Chapter 2, modifying it in order to assess the value of traceability to the dairy industry in the early stages of the production chain. Markov chain models have not previously been used in modelling for assessing traceability. We also use the concept 
of fuzzy traceability (Skoglund \& Dejmek, 2007), in relevant areas of the model. Our model provides a framework for assessing the potential value of traceability in the dairy and similar industries, filling a need to assign value to a difficult to quantify aspect of production.

\subsection{Markov Chain Model}

\subsubsection{Problem Statement}

Traceability is an important and useful addition to the quality control system of any supply chain. It is of particular interest and importance in the dairy industry, yet its value is difficult to quantify. We aim to develop a useful model for the flow of value though the dairy supply chain, incorporating traceability in such a way as to allow estimation of its value. While traceability can contribute value to a firm or industry in other ways, our model focuses on the value contributed through the reduction of product loss in the event of contamination.

\subsubsection{Product Flow DTMC Model}

Let $X(1) \longrightarrow X(2) \longrightarrow \cdots \longrightarrow X(N)$ be a Markov chain. We move through each state in the chain over time, with a uniform time step $\Delta t$ between each. The value in $X(t+1)$ depends solely on a set of transition probabilities $p(X)$ that are functions of $X(t)$ and relevant parameters. When modelling a production chain each state is a vector containing the various locations of product throughout the system.

$$
X(t)=\left[\begin{array}{c}
x_{1}(t) \\
x_{2}(t) \\
\vdots \\
x_{i}(t)
\end{array}\right] \underset{p(X)}{\stackrel{\Delta t}{\longrightarrow}} X(t+1)=\left[\begin{array}{c}
x_{1}(t+1) \\
x_{2}(t+1) \\
\vdots \\
x_{i}(t+1)
\end{array}\right]
$$

The transition probabilities that determine the product movement each time step will be unique to the specific production system being modelled.c In this chapter, we model the flow of milk from the farm to the factory. 
Milk passes through three locations after leaving the farm: tanker, factory reception, and processing. This means we have a vector of three values

$$
X(t)=\left[\begin{array}{l}
T(t) \\
F(t) \\
P(t)
\end{array}\right]
$$

where $T(t), F(t)$, and $P(t)$ are the value of product in the milk tanker, factory reception, and processing stages respectively at time $t$. Upon arrival at each location testing can occur. Using a discrete Markov chain, in which the state of the chain is the value of milk in each stage, we are able to model the milk flow and assess the value of traceability associated with each stage. Because the number of values each stage in $X(t)$ can take are finite, this is also a finite state Markov chain. The total number of possible states depends on the amount of product that is allowed to move between stages in each time step and the maximum capacity of each stage. For example, if the maximum capacity vector is given by $\left(T_{\max }, F_{\max }, P_{\max }\right)=(130,200,880)$ using a movement of one unit each time step, the total number of states is given by $130 \times 200 \times 880=22,880,000$. If we increase the movement to ten units each time step, we have $\frac{130}{10} \times \frac{200}{10} \times \frac{880}{10}=22,880$ possible states. The probability for transitioning from state $i$ to state $j$ is $P_{i j}$. We will explain the transition probabilities for the tanker, factory reception and processing stages in Sections 3.4.4, 3.4.5, and 3.4.6 respectively.

We use discrete time Markov chains because the events where milk moves between stages are clearly defined. Beginning with each equation separately, we can derive the probability of each event happening in a discrete time period. Figure 3.1 shows the path milk takes from the farm to the factory, and where the event decisions occur.

When testing is undertaken, a number of tests are performed. The results of some of these tests are available instantaneously, however some may not be available for several hours, or possibly days. Fonterra recently developed a new milk fingerprinting system that allows them to get most relevant milk quality information on the same day the milk is collected. Instantaneous results available at the farm prior to collection are those generally organoleptic tests, regarding smell appearance and temperature (Rural Delivery, 2016). 


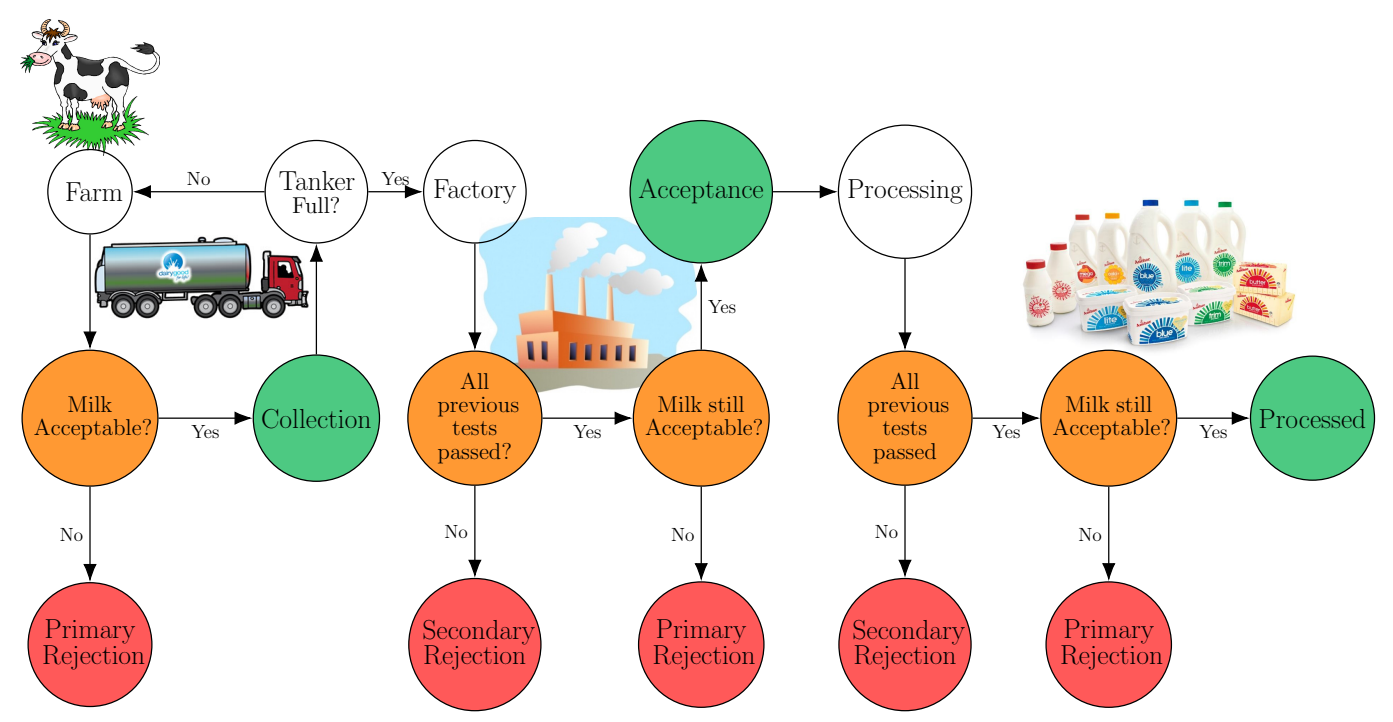

Figure 3.1: Flow chart showing the path milk takes from the farm to the factory and the decisions that are made along the way.

The results of an "instantaneous" test will determine primary rejection at whatever stage of the process the test is performed. Long term tests are those that require testing in a laboratory environment, such as tests for bacteria and chemical residues. Because the results of a long term test are not available immediately, they do not influence primary rejection in the current stage, rather they may result in a secondary rejection at a future stage.

A discrete time Markov chain models the state of a system (here the value of milk in different stages of the dairy supply chain) from one time step to the next, where each time step is the same size. In order to specify the transition probabilities, the time step must be small enough that in any given time step at most one event can occur. For example, throughout a day there are $\Phi$ collections made by milk tankers. To ensure that there is at most one collection or delivery per time step, the length of the time step must be less than $\frac{1}{\Phi}$ days. In this model the value of the time step must account for all events that can occur across the three stages (tanker, factory. and processing). Possible values for the time step will be discussed in section 3.4.7.

A continuous time Markov chain model was briefly considered for this application. The transition probabilities look fairly similar to the DTMC model, they are still defined for a small time interval $\Delta t$, but are referred to as in- 
finitesimal transition probabilities because they are valid for infinitesimally small $\Delta t$. By including terms $o(\Delta t)$ in the definitions of the infinitesimal transition probabilities, where $\lim _{\Delta t \rightarrow 0}(o(\Delta t) / \Delta t)=0$, we are able to vary the sizes of the time steps in an exponential distribution and simulate continuous time (Allen et al., 2008). We ultimately decided the DTMC model was more appropriate in this situation.

\subsubsection{Developing the Model with Traceability}

Starting with the multi-event model developed in Chapter 2, we will incorporate terms and transition probabilities to represent various aspects of traceability. In this thesis we make the assumption that any costs associated with the care and milking of the cows are the responsibility of the farmer and do not influence our model.

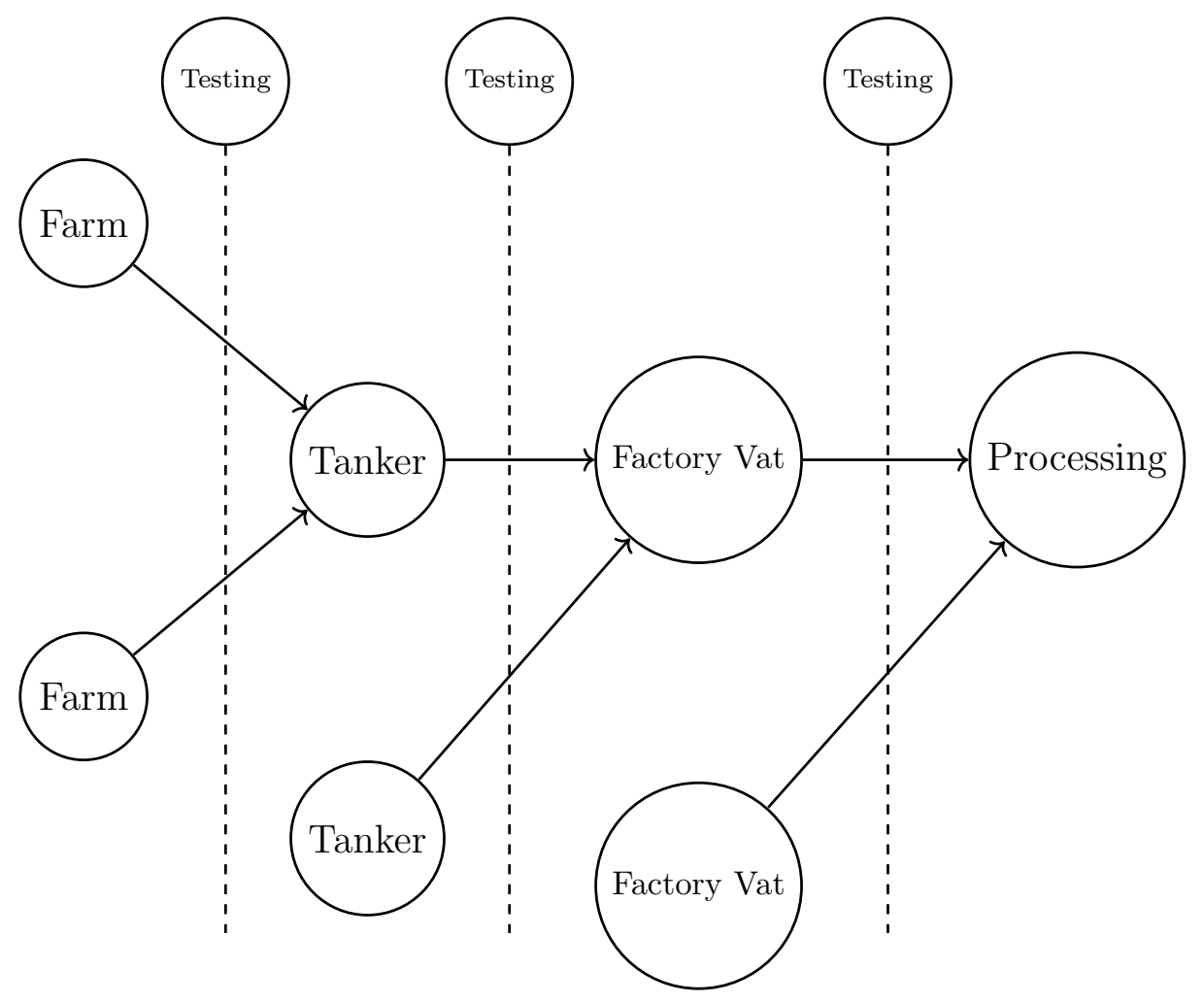

Figure 3.2: The path of milk from the farm to the factory. Each time the milk enters a new stage testing and potential rejection is depicted by vertical dashed lines.

The path the milk takes from the farm to the factory is depicted in Figure 3.2. 
There are three possible points for testing, each before milk from different sources is combined. These are prior to collection by a milk tanker, before a tanker deposits its load in a vat at the factory, and before entering processing. Raw milk will not keep for long, so cannot be held in each stage to wait for the results of any non-instantaneous (long term) test. Therefore there is an element of traceability required to keep track of where the milk has gone, should any of the tests come back with a poor result at any stage. Table 3.1 summarises the parameters we will use in this model. Frequencies are represented by the capital Greek letters $\Phi, \mathcal{X}, \Psi$ and $\Omega$. Probabilities are represented by lowercase Greek letters. Testing costs are represented by an $E$ with a subscript for the associated stage, similarly for the traceability costs using an $L$. The value of the vat on the farm, which the tankers collect from, is given by $V$, we assume this to be the same for all farms.

This model extends that presented in Chapter 2 by including terms for the cost of traceability, and new probabilities for rejecting product based on the results of previous tests, which we label as secondary rejection probabilities. The effects of traceability on product loss are also incorporated through 'traceability factors' and fuzzy traceability 'mixing errors' which we will explain in the relevant sections. As was the case in Chapter 2, the transition matrix of the Markov chain is time invariant. The probability of material moving into and out of each stage does vary with the quantity in each stage at time $t$, but this is not affected by time. This may be a bit unrealistic, but it is a modelling convenience that should not have much impact on the overall model output. Fonterra does collect and process milk 24 hours a day, but there are still likely to be times of the day that are busier than others.

\subsubsection{The Milk Tankers}

The tanker stage operates as a pool of value, into which individual collections by tankers are added and from which individual tanker delivers are removed. There are four possible events that can occur in the milk tanker stage, one at a time. They are milk collection, milk rejection, milk delivery, or transporting. Figure 3.3 is a flow diagram of the value entering and leaving the tanker stage. This is essentially a close up view of the tanker stage in Figure 3.2 In any given period of time the tanker will make a certain number of col- 


\begin{tabular}{|c|c|c|}
\hline Parameter & Description & Units \\
\hline$V$ & Amount of milk collected from an on-farm vat & $\$$ \\
\hline$\Phi$ & Frequency of collection attempts & Vats per day \\
\hline $\mathcal{X}$ & Frequency of delivery to factory & Tankers per day \\
\hline$\Psi$ & Frequency with which milk enters processing & Silos per day \\
\hline$\Omega$ & Frequency of process exit & Units per day \\
\hline$E_{T}$ & Cost of testing milk at collection site & $\$$ \\
\hline$L_{T}$ & Cost of tracing collected milk* & $\$$ \\
\hline$E_{F}$ & Cost of testing milk upon delivery & $\$$ \\
\hline$L_{F}$ & Cost of tracing accepted milk* & $\$$ \\
\hline$D_{F}$ & Cost of disposing of unwanted milk & $\$$ \\
\hline$E_{P}$ & Cost of testing prior to processing & $\$$ \\
\hline$L_{P}$ & Cost of tracing milk accepted for processing* & $\$$ \\
\hline$D_{P}$ & Cost of disposing of unaccepted silo milk & $\$$ \\
\hline$\alpha$ & Probability of primary vat acceptance & N/A (Probability) \\
\hline$\beta$ & Probability of primary tanker acceptance & N/A (Probability) \\
\hline$\gamma$ & Probability of primary silo acceptance & N/A (Probability) \\
\hline$\eta$ & Probability of secondary vat acceptance* & N/A (Probability) \\
\hline$\theta$ & Probability of no secondary rejection at processing entry* & N/A (Probability) \\
\hline$\varsigma$ & Probability of Partial primary tanker acceptance & N/A (Probability) \\
\hline$\varpi$ & Probability of Partial secondary tanker acceptance* & N/A (Probability) \\
\hline$\varrho$ & Probability of Partial silo acceptance* & N/A (Probability) \\
\hline$\lambda$ & Tanker Traceability factor* & $\mathrm{N} / \mathrm{A}(0$ or 1$)$ \\
\hline$\varepsilon$ & Mixing error for a tanker load in a reception silo* & $\$$ \\
\hline$\ell$ & Factory reception traceability factor* & $\mathrm{N} / \mathrm{A}(0<\ell<1)$ \\
\hline$C_{T}$ & Average capacity of one milk tanker & $\$$ \\
\hline$N_{T}$ & Total capacity of entire milk tanker fleet & $\$$ \\
\hline$C_{F}$ & Reception silo capacity & $\$$ \\
\hline$N_{F}$ & Total Factory reception stage capacity & $\$$ \\
\hline$N_{P}$ & Total Processing capacity & $\$$ \\
\hline$Q$ & Value leaving Processing each time step & $\$$ \\
\hline
\end{tabular}

Table 3.1: A summary of each parameter used in our model and their units.

lections and a certain number of deliveries. In Figure 3.3 the frequency of collection (the number of collection attempts per day) is represented by $\Phi$, while delivery frequency is denoted by $\mathcal{X}$. The probability that the milk being collected passes all 'instantaneous' tests on site, and is mixed with the product already in the tanker is given by $\alpha$, meaning $1-\alpha$ is the probability that an instantaneous test is failed and the milk is rejected. $T(t)$ is the value contained in all of the tankers at time $t$. $V$ represents the value of the farm vat that is transferred to the milk tanker, this is a constant as we assume that every collection the same amount of material is collected from every farm. If there is no testing prior to collection by the tanker, $\alpha=1$ as the milk cannot be rejected, and $E_{T}=0$ as there is no cost of testing. 


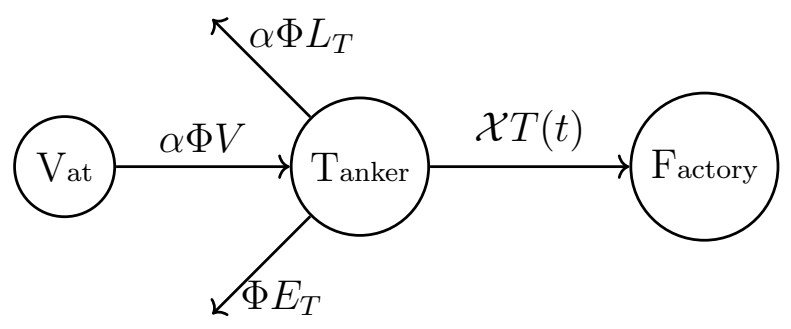

Figure 3.3: The flow of value into and out of the tanker stage. $T(t)$ is the value in the tanker stage at time $t$.

The tanker will make its delivery to the factory prior to receiving the results of some tests, we refer to these tests as 'long term' tests. These are the tests that may lead to secondary rejections upon entry to the factory reception or processing stages, depending on how long the results take. What happens to the milk after this point occurs at the factory reception stage, it does not affect the value in the tanker. The cost of tests is incurred, independent of the milk being collected or rejected, this cost is assumed to be constant and is represented by $E_{T}$. The cost of tracing the milk is represented by $L_{T}$, this cost only applies if the milk passes immediate testing and is collected. Because this is the first stage, no previous testing has been done, so this cost is the only modification to the milk tanker stage from the model as it is presented in Chapter 2. If milk is rejected, the co-operative does not pay the farmer for that milk, it is considered as if the milk was never supplied. The responsibility of disposing of milk rejected at this stage lies with the farmer (Fonterra Co-operative Group, 2014b).

In our model we only allow one event out of collection, rejection, or delivery, to take place in any given time step. In reality, as there are many milk tankers, two or more tankers may collect milk from different farms simultaneously. These events also take time in reality however. Because, in our model, it is possible for two events to occur in two adjacent time steps, we can closely mimic simultaneous events for different tankers.

Collection: The milk becomes the responsibility of the factory when it is collected by the tanker. This is also when the first test can be applied, before mixing with any previously acquired milk already in the tanker. For tests that yield instantaneous results, this is enough, but if any time is required 
to get the results, we need some ability to track where the milk associated with each test has gone. Because the nature of raw milk does not allow it to be kept long enough to wait for any 'long term' test results, the milk must be collected, unless any instantaneous tests suggest rejection. The results of any long term test results (when they come through) now apply to all of the milk in that tanker. The probability of an attempted milk collection in time step $\Delta t$ is given by

$$
\left(1-\frac{T(t)}{N_{T}}\right) \Phi \Delta t
$$

The probability given in Equation 3.1 is proportional to the unused capacity of the tanker stage at time $t$. In the event that all of the milk tankers are full, Equation 3.1 reduces to zero, reflecting the fact that milk cannot be collected if there is no capacity to collect it. As the tanker stage gets close to capacity, Equation 3.1 gets very small. In reality tankers are collecting milk and delivering it throughout their shift, and it is unlikely that the system approaches capacity at all. It is however logical that the closer the system is to capacity, the fewer tankers have any space to collect milk, reducing the chances of a collection at that point in time.

If all the milk tankers are empty in a given time step $t$, Equation 3.1 is reduced to $\Phi \Delta t$. It is possible $\Delta t$ will be such that $\Phi \Delta t=1$ or very close. While this may seem a little unrealistic at first, in the scenario where all tankers are empty, none will be delivering to the factory as all will be at some point on their journey from the factory to a farm. It is plausible given a large fleet of tankers that at least one tanker will be ready to collect milk in any given time step particularly under the restrictions of this scenario. Given $\alpha$ is the probability the milk passes all of the instantaneous tests and is thus is accepted by a tanker, the probability that a milk tanker collects milk in a given time step $\Delta t$ is

$$
\alpha\left(1-\frac{T(t)}{N_{T}}\right) \Phi \Delta t
$$

In transferring the milk from the farm's vat to the tanker, the value of that milk is transferred to the tanker. The tanker gains the value of the milk, but loses the cost of any testing performed as well as the cost associated with 
tracing the milk in case of poor test results in the future. The value change in the tanker stage in the event of collection is thus $V-L_{T}-E_{T}$.

Rejection. If an 'instantaneous' test is failed before the milk is added to the tanker, the milk is rejected and the tanker incurs the cost of testing, no value is gained. The probability of a rejection is given by

$$
(1-\alpha)\left(1-\frac{T(t)}{N_{T}}\right) \Phi \Delta t
$$

This results in a value change of $-E_{T}$. Rejected milk leaves the supply chain, so there is no need to trace it further meaning there is no traceability cost associated with this event.

Delivery. After collecting milk from multiple farms, the tanker will deposit its load at a factory, along with all the value associated with it. The probability that a tanker will deliver to the factory in a given time step is dependent on the amount of milk in the tanker stage $T(t)$, the number of deliveries to the factory per day $\mathcal{X}$, and the available capacity in the factory reception stage $\left(1-\frac{F(t)}{N_{F}}\right)$. The probability of a tanker delivering milk to the factory in time step $\Delta t$ is given by

$$
\frac{T(t)}{N_{T}}\left(1-\frac{F(t)}{N_{F}}\right) \mathcal{X} \Delta t
$$

At most one tanker can deliver to the factory in a given time step, so the value of milk in the tanker stage will reduce by $C_{T}$, the capacity of one tanker. We make the assumption that tankers only attempt to deliver milk to the factory if they are full. Costs associated with testing and traceability are taken out at the factory reception stage.

Transporting: If a tanker is not collecting, rejecting, or delivering, it is assumed to be in the process of travelling between farms or back to the factory. Therefore the probability that all tankers are transporting is

$$
1-\left[\left(1-\frac{T(t)}{N_{T}}\right) \Phi+\frac{T(t)}{N_{T}}\left(1-\frac{F(t)}{N_{F}}\right) \mathcal{X}\right] \Delta t
$$


When all milk tankers are transporting there is no value change in the milk tanker stage.

\subsubsection{The Factory Reception Stage}

The next stage is the reception silo at the factory where the tankers deposit their loads. The model allows for multiple dairy processing sites. As with the tankers we will be treating these as part of the factory reception pool but only one silo worth of product can be passed on to the processing stage in any single time step. The possible events in the factory reception stage are collection, rejection, passing on for processing, or holding. Figure 3.4 is a flow diagram of the value entering and leaving the factory reception stage. Once milk is collected by a tanker it become the responsibility of the dairy company, therefore if milk is rejected at the factory reception stage there is a disposal cost, $D_{F}$, that needs to be taken into account.

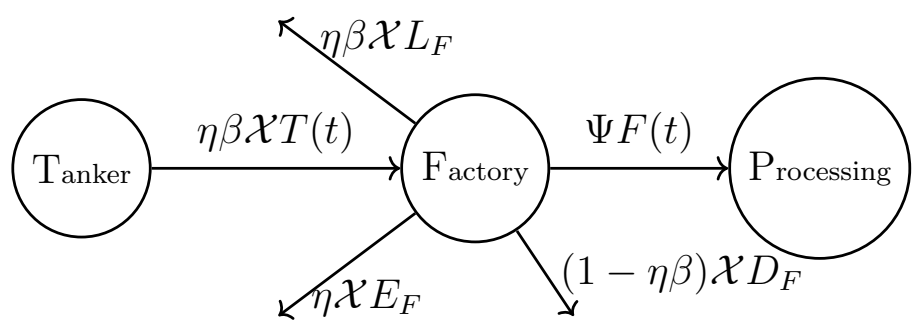

Figure 3.4: The rate of value flow into and out of the factory reception stage.

Including traceability means this stage has two possible rejection scenarios, which we will refer to as primary and secondary rejections. A primary rejection occurs when the milk is rejected due to the results of instantaneous testing conducted when the tanker arrives at the factory. The cost of the test $E_{F}$ is incurred along with the disposal cost $D_{F}$. If milk is rejected due to previous long term test results, then a secondary rejection occurs. There is no testing cost associated with a secondary rejection, as this cost has already been incurred at an earlier stage. Each milk tanker has both a trailer and a truck compartment. If these two compartments can be kept separate they can be tested, accepted and rejected separately. If we know which farms the milk in each compartment came from, then unsatisfactory test results 
that become known between collection and delivery can result in just the rejection of the affected compartment. We define $\lambda$ as the traceability factor, having a value of 1 or 0 depending on the whether we can distinguish between individual compartments on a tanker or not.

$$
\lambda=\left\{\begin{array}{l}
1, \text { with sufficient traceability } \\
0, \text { without sufficient traceability }
\end{array}\right.
$$

Milk that is accepted into the factory reception stage moves on to be processed as required. In this model milk leaves the factory reception stage at a rate of $\Psi$ units per day.

Figure 3.5 shows a probability tree for how the probability of each acceptance and rejection combination can be calculated. The rejection of a tanker compartment is denoted by $\mathbf{r}$, while a denotes its acceptance up to that point. We end up with three main possibilities; total acceptance, partial acceptance, or total rejection. Introducing traceability gives us many more pathways and potential outcomes compared with those presented in Chapter 2. When there is insufficient traceability $(\lambda=0)$ as we cannot distinguish between the individual tanker compartments until instantaneous tests are conducted upon arrival at the factory reception. In this case milk cannot be rejected due to previous 'long term' tests and the model for the factory reception stage reverts to that presented in Chapter 2.

The probability that one tanker compartment needs to be rejected is not totally independent of the other compartment's status. For example, if each tanker is collecting from three farms, the first farm's milk should fit comfortably in the first tanker compartment. Milk from the second farm visited will also be pumped into the first tank as well. It is likely, that there is not enough room in the first tanker compartment, hence milk from the second farm will be then pumped into the second tank. Even if the milk from the second farm fits fully into the first tank, any residue left in the pipe will likely be from that farm and have the potential to contaminate the next load of milk pumped through it into the second tank. In the case of an even number of farms $(2 n)$, the farm potentially contaminating both tanks will be the nth farm. For example, with four farms this will be the second farm. If, after milk collection is complete, we learn that one of the tanks on the tanker now 


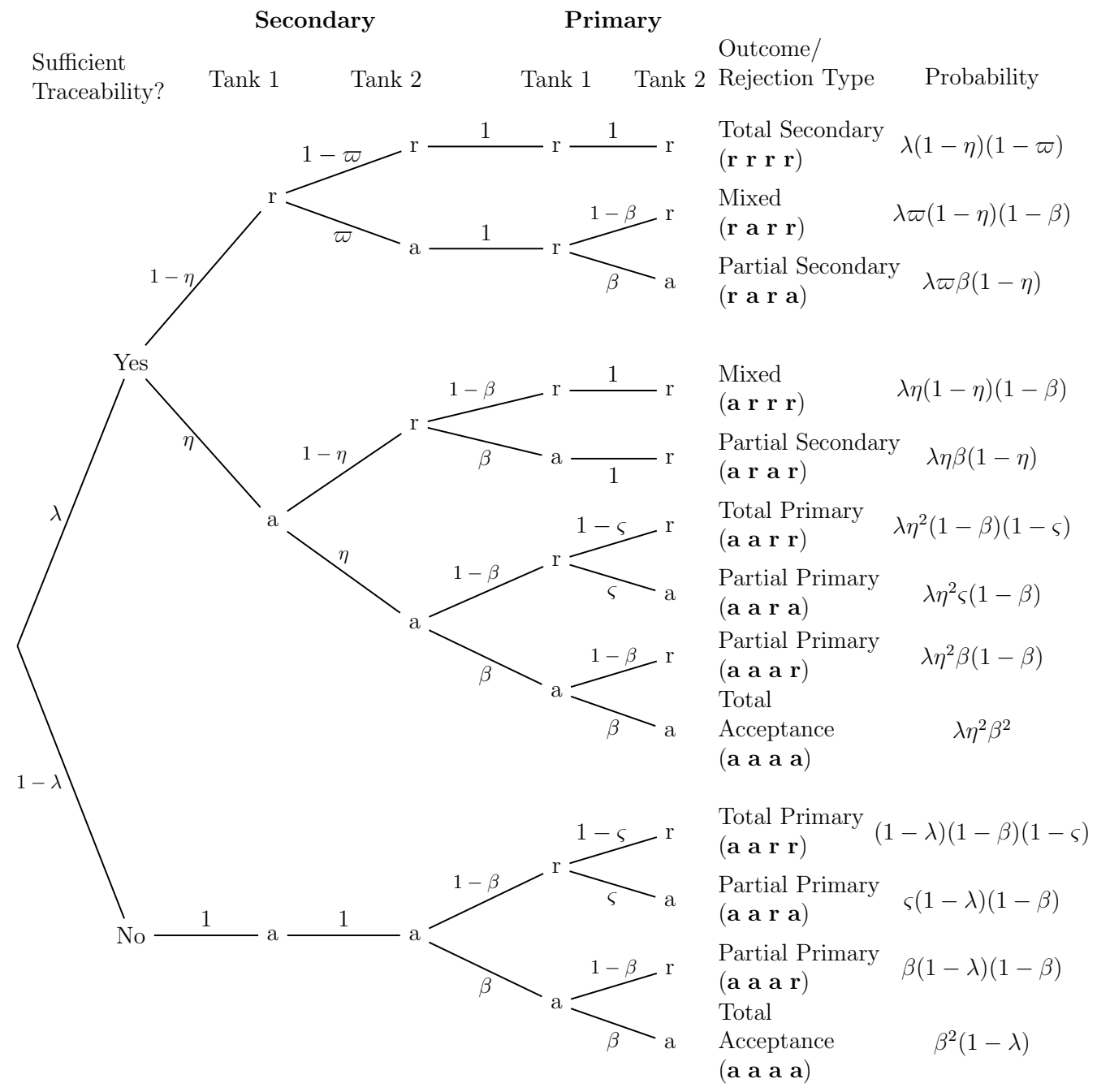

Figure 3.5: Probability tree showing the possible outcomes when milk is delivered to the factory.

contains contaminated milk, given that only two of the farms contributed milk to this tank, there is a $50 \%$ chance that this was the second farm in the collection run. There is the possibility that each tanker compartment is contaminated independently of the other by a different farm, with probability $\eta$. In figure 3.5, these conditional acceptance probabilities are represented by $\varsigma$ and $\varpi$ for primary and secondary rejections respectively. The example presented above would imply $\varsigma=0.5 \beta$ and $\varpi=0.5 \eta$.

Total acceptance. In this event both tanker compartments pass all tests (both instantaneous and long term) up to this point and are accepted. There- 
fore the probability of a milk delivery being accepted at the factory reception stage is simply the probability of a delivery attempt multiplied by the probability that the milk is not rejected in either a primary or secondary rejection scenario. The probability of a delivery attempt is given in Equation 3.2. The acceptance probabilities are given in Figure 3.5 as $\lambda \eta^{2} \beta^{2}$ and $\beta^{2}(1-\lambda)$. Thus the probability of total acceptance, given that delivery is attempted, is given by

$$
\left[\lambda \eta^{2} \beta^{2}+\beta^{2}(1-\lambda)\right]=\beta^{2}\left[1+\lambda\left(\eta^{2}-1\right)\right]
$$

where $\beta$ is the probability of a tanker compartment passing all instantaneous tests conducted at this stage (primary acceptance), and $\eta$ is the probability that no previous long term tests have detected any contamination (secondary acceptance). The resulting value change is

$$
C_{T}-E_{F}-L_{F}
$$

where $C_{T}$ is the average value of milk in a tanker, $E_{F}$ is the cost of conducting tests at this stage and $L_{F}$ is the traceability cost incurred by accepted milk.

Partial acceptance. This occurs when just one tanker compartment is accepted by the factory. The other compartment could be rejected in either the primary or secondary rejection scenario. This will result in a slightly different value change, due to the testing costs only associated with a primary rejection.

A Primary partial rejection occurs with probability

$$
\begin{aligned}
& {\left[\lambda \eta^{2} \varsigma(1-\beta)+\lambda \eta^{2} \beta(1-\beta)+\varsigma(1-\lambda)(1-\beta)+\beta(1-\lambda)(1-\beta)\right]} \\
& =(1-\beta)(\varsigma+\beta)\left[1+\lambda\left(\eta^{2}-1\right)\right]
\end{aligned}
$$

This is the sum of the probabilities that the rejection occurs in either tank as given in Figure 3.5. The order of rejection has no effect on the outcome in this case. Because only one tanker compartment is rejected, half of the milk that was delivered is added to that currently contained in the factory reception stage, where as he full testing and tracing costs still apply. The value change in the Factory reception stage in the case of a primary partial 
rejection is therefore

$$
\frac{C_{T}-D_{F}}{2}-L_{F}-E_{F}
$$

The probability of a Secondary partial rejection is given by

$$
[\lambda \varpi \beta(1-\eta)+\lambda \eta \beta(1-\eta)]=\lambda \beta(1-\eta)(\varpi+\eta)
$$

A secondary partial rejection may occur when the results of a previous long term test require one tanker compartment to be rejected. Because the milk has been rejected, no further testing is required for that tank. Testing is still required for the other tank which incurs a cost of $\frac{E_{F}}{2}$. The resulting total value change is

$$
\frac{C_{T}-E_{F}-D_{F}}{2}-L_{F}
$$

Total rejection. There are three ways a total rejection could occur. Both tanks could be rejected by previous long term tests in a total secondary rejection, both by instantaneous tests in a total primary rejection, or one of each resulting in a composite rejection.

A Total primary rejection requires both tanks to pass all earlier testing, then both be rejected due to the results of testing on arrival at the factory. The probability of this occurring is given by

$$
\begin{aligned}
& {\left[\lambda \eta^{2}(1-\beta)(1-\varsigma)+(1-\lambda)(1-\beta)(1-\varsigma)\right]} \\
& =(1-\beta)(1-\varsigma)\left(1+\lambda\left(\eta^{2}-1\right)\right)
\end{aligned}
$$

The value change in this case is the cost of testing and disposing of both tanker compartments

$$
-E_{F}-D_{F}
$$

In a Total secondary rejection both compartments are rejected due to earlier long term testing. The resulting value change consists solely of the disposal cost $-D_{F}$. The probability of this scenario occurring is given by

$$
\lambda(1-\eta)(1-\varpi)
$$


There is also the chance that one tanker compartment will be rejected based on earlier long term tests, while the other is rejected by instantaneous tests conducted upon arrival at the factory, resulting in a Composite rejection scenario. The probability of this happening in a given time step is

$$
\begin{aligned}
& {[\lambda \varpi(1-\eta)(1-\beta)+\lambda \eta(1-\eta)(1-\beta)] } \\
= & \lambda(1-\eta)(1-\beta)(\varpi+\eta)
\end{aligned}
$$

The change in the factory reception stage in this case is

$$
\frac{-E_{F}}{2}-D_{F}
$$

In this scenario there are no traceability costs incurred as no milk is accepted. Because of the within compartment mixing that will take place during tanker transport, the main contribution made by traceability at the factory reception stage is reduced testing costs. The traceability implemented up to and at this stage will have an impact on the precision possible in later stages.

Passing on. Passing material on to the initial processing stage is the other possible event that can occur in a given time step. The probability that milk will leave the factory reception stage and move on for processing is dependent on the value of milk contained in the factory reception stage. and is given by

$$
\frac{F(t)}{N_{F}} \Psi \Delta t
$$

There is now an upper limit on the movement each time step based on the capacity of a reception silo, $C_{F}$. The change in value in the factory reception stage when milk is passed on for processing is $-C_{F}$, the average capacity of a reception silo.

\subsubsection{The Processing Stage}

Here we look at how the value contained in the processing stage is changing over time. Processing of milk begins with separation, followed by standardisation. After these steps every dairy product undergoes pasteurisation (Hudson et al., 2003; Western, 2011). The possible events include collection, 
rejection, passing on, and production. In this part of the model we also have both primary and secondary rejection and both result in a disposal cost. Only primary rejection incurs any testing cost, as secondary rejection is dependent on previous tests. After milk has undergone the initial processing stages of separation, standardisation and pasteurisation, it is passed on to different production processes depending on the intended end product. These further steps of processing are not included in our model.

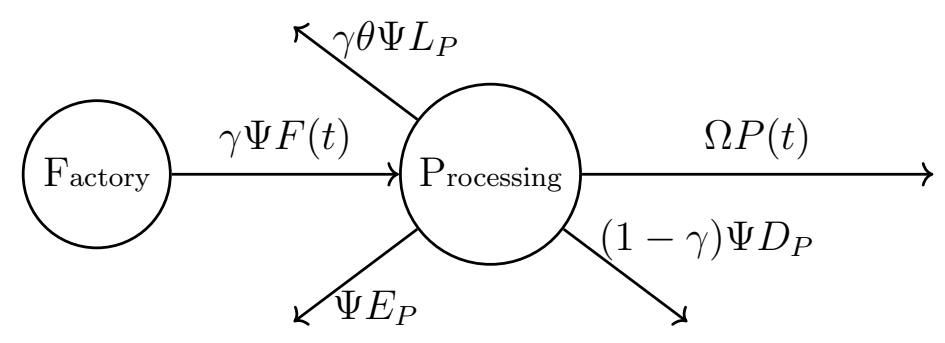

Figure 3.6: The rate of value flow into and out of the processing stage.

At this stage of the production chain, the level of traceability can determine how much product is lost in the case of a contamination. With perfect traceability we will know which tanker, and which tank on this tanker, the unsatisfactory material came from. We will also know what time it entered the reception silo and how much milk went in before and after it. A certain degree of mixing will occur, so we must allow for this, but it should be possible to reject only the unsatisfactory tank load along with a mixing allowance either side of this. Figure 3.7 shows a probability tree for the possible outcomes when milk enters this stage. Primary rejection via 'instantaneous' test results occurs with probability $\gamma$. The probability that a contamination is detected via a 'long term' test from a previous stage is given by $\theta$. Depending on how much of the silo may be salvageable, and if this is worth more than the costs associated with accepting it, $\mu$ takes the value zero or one. Retesting of the remaining product is necessary in this case to ensure any contamination has been successfully removed. The probability that a test of salvaged product detects contamination is given by $\varrho$. 


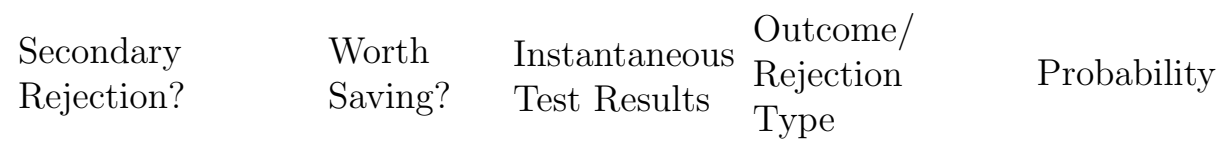

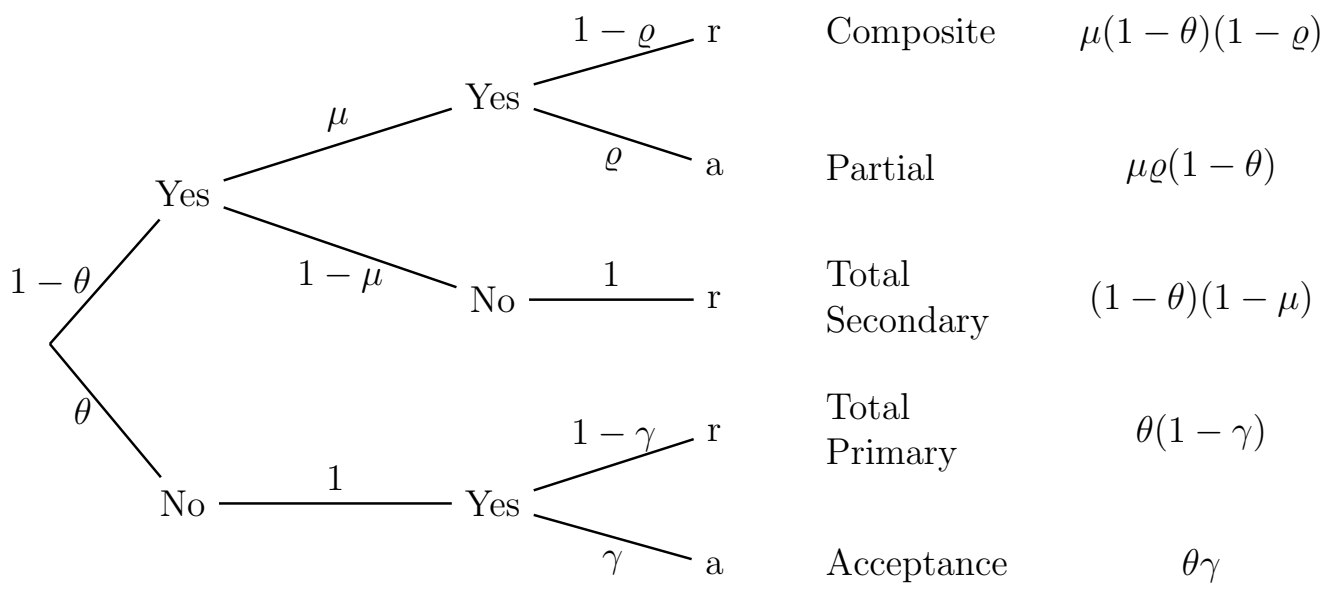

Figure 3.7: Probability tree for the outcomes when passing product from the factory reception stage to processing.

Acceptance. The probability that all of the milk in a reception silo is accepted for processing is

$$
\frac{\theta \gamma F(t)}{N_{F}} \Psi \Delta t
$$

The value change, now limited by the capacity of a reception silo, becomes

$$
C_{F}-E_{P}-L_{P}
$$

where $E_{P}$ is the cost associated with testing at this stage and $L_{P}$ is the traceability cost.

Partial Acceptance. This occurs when some milk is able to be salvaged following a secondary rejection. This is the event in the processing stage where, we apply the concept of fuzzy traceability (Skoglund \& Dejmek, 2007) and the impact of traceability implemented in the previous stages is seen We allow that some milk may occupy the same reception silo as contaminated milk without becoming contaminated itself. This can be due to timing in the entry and exit of product into the silo, or the nature of the contamination 
may mean it is easily removable or able to be diluted to levels where it is no longer harmful.

In the secondary rejection scenario, we are dealing with test results that have come from either the tanker collection or the factory reception stage. For a secondary rejection to be possible in the processing stage $\theta$ must be less than one. The closer $\theta$ is to zero, the more likely we are to reject product via secondary rejection. The value change in this scenario largely depends on the traceability in previous stages. If a secondary rejection occurs it means some of the milk in this silo has come from a tanker identified as containing contaminated milk after it delivered its load. If we have sufficient traceability at the tanker level $(\lambda=1)$ we will be able to identify which compartment of the affected tanker contained the contaminated milk. Remember $\lambda=0$ when traceability is insufficient to distinguish between individual tanks on a milk tanker. The level of traceability at the factory reception level, $\ell$, determines how precisely we can locate the contaminated tank load within the silo, $\ell$ must be between zero and one. If $\ell=1$, we have perfect traceability and can identify the contaminated load exactly. Obviously, since milk is liquid some mixing will occur. We model this mixing error using the parameter $\varepsilon$. This can take any value from zero, implying no mixing error, to $C_{F}(1+\lambda)-C_{T}$, where one contaminated tank load contaminates the whole silo. Once the contaminated milk is removed from the silo, the remaining product left in the silo is given by

$$
\ell\left(C_{F}-\frac{C_{T}+\varepsilon}{1+\lambda}\right)
$$

The $1+\lambda$ in the denominator becomes either two or one depending on whether we can just reject one tank, or must reject the whole tanker load. Equation 3.3 assumes that only one contaminated taker load may be identified in a reception silo. While it is theoretically possible that there may be more than one contaminated load in a single silo, for the sake of simplicity in our model we only allow for one to be the cause of a partial rejection. The actual incidence of contamination will generally be low enough that we can safely deal with any potential multiple contaminations through the composite rejection scenario described in Equation 3.5.

The value contained in the processing stage will increase by the amount of 
milk accepted and decrease by the costs of retesting, tracing and disposal. We also assume the test cost $E_{P}$, and the tracing cost $L_{P}$ are the same regardless of the volume being tested. The probability of a partial acceptance is given by

$$
\frac{\mu \varrho(1-\theta) F(t)}{N_{F}} \Psi \Delta t
$$

A disposal cost of $D_{P}$ is incurred for each unit of milk that must be discarded. The total value change to the processing stage, in the event of a partial secondary rejection is

$$
\begin{aligned}
& \ell\left(C_{F}-\frac{C_{T}+\varepsilon}{1+\lambda}\right)-\left(C_{F}-\ell\left(C_{F}-\frac{C_{T}+\varepsilon}{1+\lambda}\right)\right) D_{P}-E_{P}-L_{P} \\
& =\ell\left(C_{F}-\frac{C_{T}+\varepsilon}{1+\lambda}\right)\left(1+D_{P}\right)-C_{F} D_{P}-E_{P}-L_{P}
\end{aligned}
$$

In order for the partial acceptance to be cost effective, Equation 3.4 must be greater that 0 . Therefore

$$
\mu= \begin{cases}0, & \text { for } \ell\left(C_{F}-\frac{C_{T}+\varepsilon}{1+\lambda}\right) \leq \frac{C_{F} D_{P}+E_{P}+L_{P}}{1+D_{P}} \\ 1, & \text { for } \ell\left(C_{F}-\frac{C_{T}+\varepsilon}{1+\lambda}\right)>\frac{C_{F} D_{P}+E_{P}+L_{P}}{1+D_{P}}\end{cases}
$$

This value that is salvaged must still undergo instantaneous testing before entry into the processing stage and may potentially be rejected, resulting in a composite rejection.

\section{Total rejection}

There are three different scenarios in this stage that could lead to total rejection; primary rejection, secondary rejection (where any salvageable milk is not worth the cost), or a composite rejection where some milk is salvaged following a secondary rejection but fails subsequent testing.

The probability of a Primary rejection is given by

$$
\frac{\theta(1-\gamma) F(t)}{N_{F}} \Psi \Delta t
$$

In the case of a primary rejection, it is not known when the unsatisfactory 
material entered the silo as no previous tests have picked it up. The whole reception silo is lost and the value change becomes $-E_{P}-D_{P}$.

In a Secondary rejection, traceability will determine what proportion of the reception silo must be disposed of. If the value of milk to be accepted following a partial rejection is less than the costs associated with accepting it, the whole silo will be rejected. The probability of this occurring is

$$
\frac{(1-\theta)(1-\mu) F(t)}{N_{F}} \Psi \Delta t
$$

The value change in this scenario is $-D_{P}$.

The probability of the retest detecting a problem is $(1-\varrho)$, thus a Composite rejection will occur with probability

$$
\frac{\mu(1-\theta)(1-\varrho) F(t)}{N_{F}} \Psi \Delta t
$$

In this case we lose both the costs of testing and disposal, $-E_{P}-D_{P}$.

\section{Passing on:}

The probability that material is passed on from this processing stage is given by

$$
\Omega \Delta t
$$

There are no costs associated with passing material on to the next stage, but the value of material in the processing stage will decrease by $Q$, the value of milk that moves in each passing on event.

\section{Producing}

If no milk is coming into or leaving the processing stage, we assume that all factories are busy producing processed milk ready to pass on to the next stage. The probability that all the factories are producing is the probability 
that none of them are doing anything else, that is

$$
1-\left[\frac{F(t) \Psi}{N_{F}}+\Omega\right] \Delta t
$$

When all factories are producing only, there is no value change in the processing stage.

\subsubsection{Summary equations and Stage Interactions:}

The following equations in this section summarise the probabilities of the outcomes described in Sections 3.4.4, 3.4.5, and 3.4.6 and how the events in each stage impact the value contain in all of the stages.

Equation 3.6 summarises the transition probabilities, and resulting value changes, for the tanker stage, where $T(t)=f$ is the value of milk in the stage at time $t$ and $g$ is the value change due to the event that takes place in this stage during the time step $t+\Delta t$.

Here $m$ represents the value change that takes place in the factory reception stage given delivery. The value in the factory reception stage is also influenced by what happens in this stage, and changes by the value of $j$. When the tanker delivers to the factory reception stage, the milk still needs to accepted or rejected in some combination. This is referred to as a value change of $m$ in Equation 3.6, the values for which are defined in Equation 3.7. The sum of these four transition probabilities equals one, because these transitions represent all possible changes in the tanker stage over the time interval $\Delta t$.

Equation 3.8 summarises the transition probabilities for the factory reception stage. The event that takes place in this stage during a given time step will also influence the value in either the tanker stage or the processing stage. The probability of accepting or rejecting a delivery is dependent on there being a delivery in the first place, which occurs with probability $\frac{T(t)}{N_{T}}\left(1-\frac{F(t)}{N_{F}}\right) \mathcal{X} \Delta t$ and implies value has left the tanker stage.

Where $n$ represents the value change that takes place in the processing stage given that the factory reception stage is passing on. When the factory reception stage passes material on the the processing stage, the milk is either accepted or rejected in some combination. This is referred to as a value 
$\overparen{0}$
0

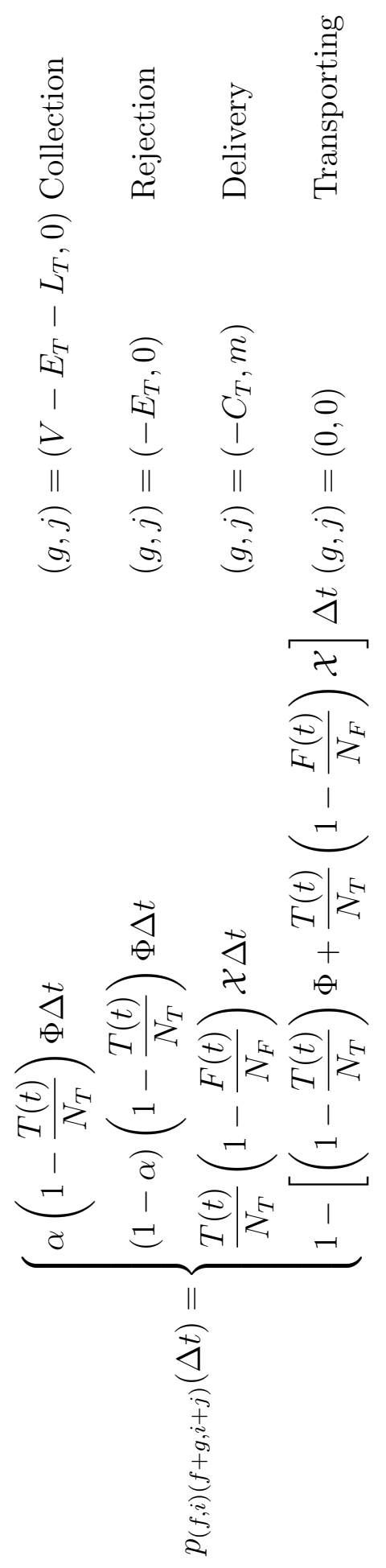



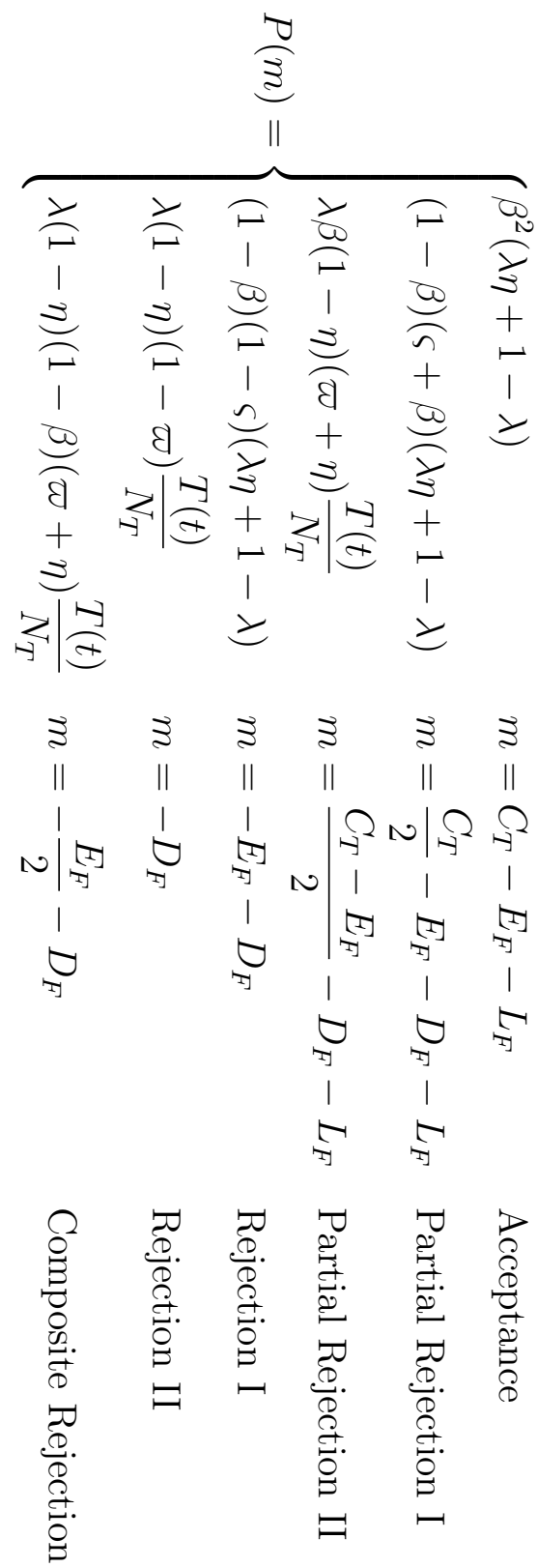


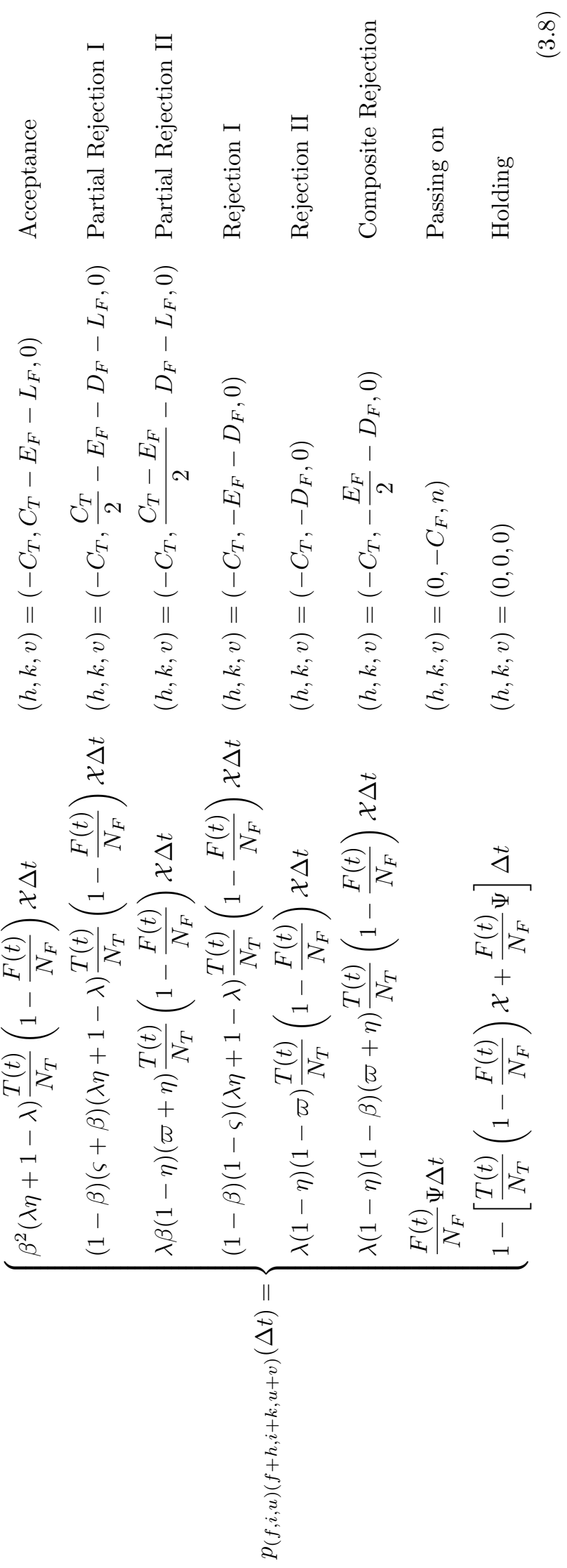


change of $n$, the possible values for which are defined in Equation 3.9.

In the processing stage, $u$ is the amount of product in this stage at time $t$ while $w$ is the value change that occurs due to the event that takes place in time step $\Delta t$. Equation 3.10 shows the probability of each possible value of $w$ given the starting value $u$. The value in the factory reception stage also changes, as acceptance or rejection by the processing stage requires value to have left the factory reception stage.

At the beginning of each time step we start with $(T(t), F(t), P(t))=(f, i, u)$. Once all of the events in all stages are complete these values are updated to $(T(t), F(t), P(t))=(f+g+h, i+j+k+s, u+v+w)$, as defined in the transition probabilities above.

\section{Time Step Size}

The time step is chosen to ensure that all of the transition probabilities, within each stage add to one. Individually each probability must be between zero and one in any given time step. In the case of the tanker stage this means we require:

$$
\left[\left(1-\frac{T(t)}{N_{T}}\right) \Phi+\frac{T(t)}{N_{T}}\left(1-\frac{F(t)}{N_{F}}\right) \mathcal{X}\right] \Delta t \leq 1
$$

There is no global maximum for the left hand side of Equation 3.11 but depending on the relative sizes of $\Phi$ and $\mathcal{X}$ we can estimate local maximums and then solve for the maximum allowable value of $\Delta t$. For $\Phi \geq \mathcal{X}$ the left hand side of Equation 3.11 is maximised when $T(t)=0$ giving

$$
\begin{array}{r}
{\left[(1-0) \Phi+0\left(1-\frac{F(t)}{N_{F}}\right) \mathcal{X}\right] \Delta t \leq 1} \\
\Longrightarrow \Phi \Delta t \leq 1 \\
\Longrightarrow \Delta t \leq \frac{1}{\Phi}
\end{array}
$$

If $\Phi<\mathcal{X}$, the left hand side of Equation 3.11 is maximised when $T(t)=N_{T}$ 


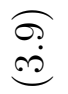
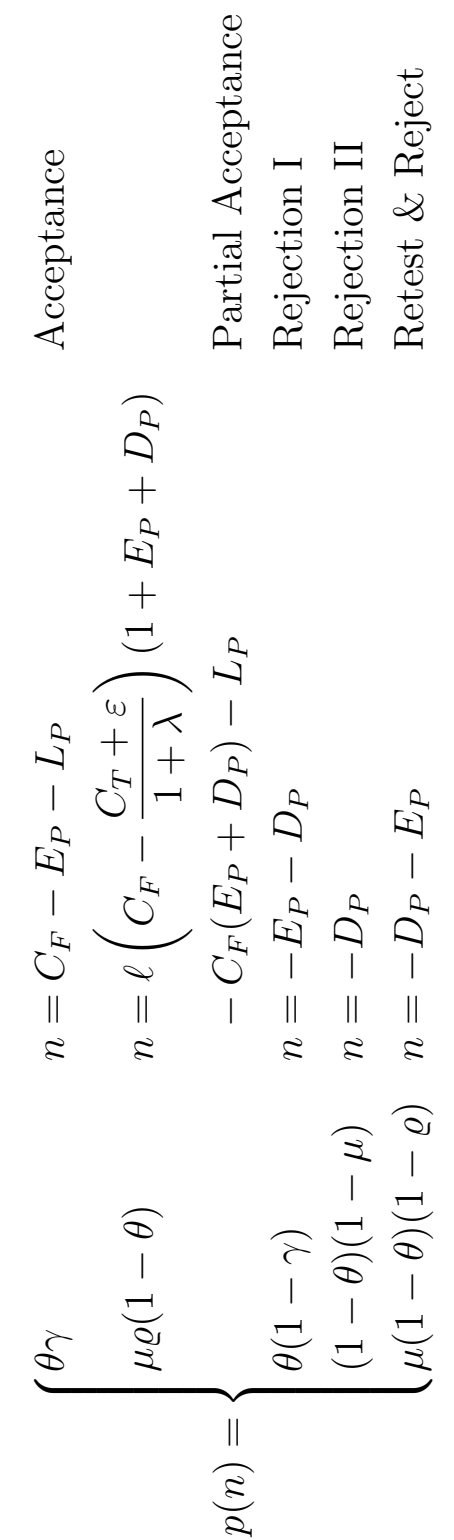


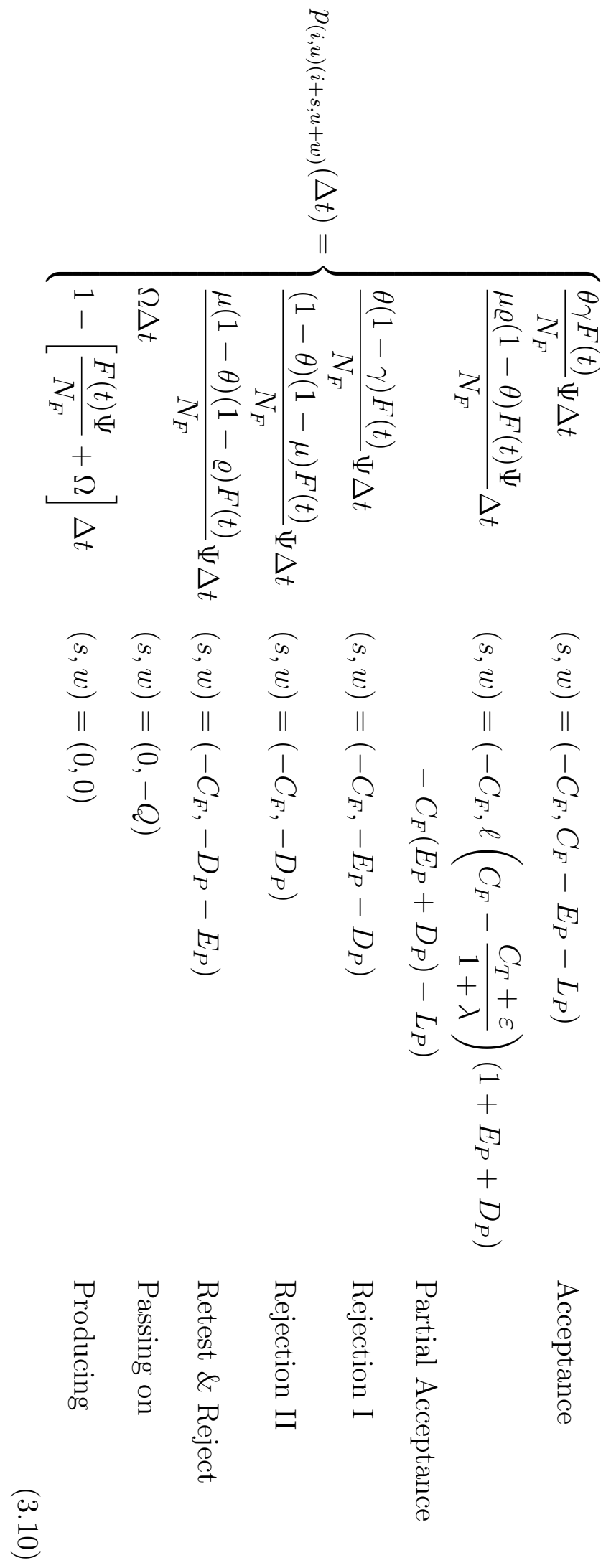


and $F(t)=0$ giving

$$
\begin{array}{r}
{\left[\left(1-\frac{N_{T}}{N_{T}}\right) \Phi+\frac{N_{T}}{N_{T}}(1-0) \mathcal{X}\right] \Delta t \leq 1} \\
\Longrightarrow 0+\mathcal{X} \Delta t \leq 1 \\
\Longrightarrow \Delta t \leq \frac{1}{\mathcal{X}}
\end{array}
$$

Combining the two we can write

$$
\Delta t \leq \frac{1}{\max \{\Phi, \mathcal{X}\}}
$$

For the factory reception stage, the time step size must be small enough that the transition probabilities in Equation 3.8 are each less than one, and all sum to one, allowing only one event to take place in each time step. This means we need to choose $\Delta t$ such that:

$$
\left[\frac{T(t)}{N_{T}}\left(1-\frac{F(t)}{N_{F}}\right) \mathcal{X}+\frac{F(t)}{N_{F}} \Psi\right] \Delta t \leq 1
$$

Again there is no global maximum for the left hand side of Equation 3.13 but depending on the relative values of $\mathcal{X}$ and $\Psi$ we can estimate local maximums and then solve for the maximum allowable value of $\Delta t$.

For $\Psi \geq \mathcal{X}$ the left hand side of Equation 3.13 is maximised when $T(t)=0$, and $\mathcal{X}=N_{F}$ giving

$$
\begin{array}{r}
{\left[0\left(1-\frac{N_{F}}{N_{F}}\right) \mathcal{X}+\frac{N_{F}}{N_{F}} \Psi\right] \Delta t \leq 1} \\
\Longrightarrow \Psi \Delta t \leq 1 \\
\Longrightarrow \Delta t \leq \frac{1}{\Psi}
\end{array}
$$

If $\Psi<\mathcal{X}$, the left hand side of Equation 3.13 is maximised when $T(t)=N_{T}$ and $F(t)=0$ giving

$$
\begin{array}{r}
{\left[\frac{N_{T}}{N_{T}}(1-0) \mathcal{X}+0 \Psi\right] \Delta t \leq 1} \\
\Longrightarrow 0+\mathcal{X} \Delta t \leq 1
\end{array}
$$




$$
\Longrightarrow \Delta t \leq \frac{1}{\mathcal{X}}
$$

Combining the two we can write

$$
\Delta t \leq \frac{1}{\max \{\Psi, \mathcal{X}\}}
$$

As in both the tanker and factory reception stages, $\Delta t$ must be small enough that only one event can take place in the processing stage in any time step. The transition probabilities in Equation 3.10 must all sum to one, and individually have a value between zero and one. In the case of the processing stage this reduces to

$$
\left[\frac{F(t) \Psi}{N_{F}}+\Omega\right] \Delta t \leq 1
$$

The left hand side of Equation 3.15 is maximised when $F(t)=N_{F}$, giving

$$
\begin{gathered}
\left(\frac{N_{F} \Psi}{N_{F}}+\Omega\right) \Delta t \leq 1 \\
\Longrightarrow \Delta t \leq \frac{1}{\Psi+\Omega}
\end{gathered}
$$

The final time step value chosen must fit the restrictions derived in all three stages, given in Equations 3.12, 3.14 and 3.16. This means $\Delta t$ will be such that

$$
\Delta t \leq \frac{1}{\max \{\Phi, \mathcal{X},(\Psi+\Omega)\}}
$$

\subsection{Model Simulation Results and Discussion}

We have developed and described the model in three separate stages. While each stage has its own set of transition probabilities, the stages all interact with each other and affect how each other's transition probability values change from time step to time step. The state vector for time $t$ is $(T(t), F(t), P(t))=(i, u, g)$, this becomes $(j, v, h)$ over the time step $\Delta t$. In this section we simulate the model developed in Section 3.4 to investigate the effects of traceability. It is assumed that the dairy producer wants to minimise product loss due to contamination, and thus maximise overall product output. Chapter 2 explores the value of milk flow from the farm 
to the factory. This chapter extends this model by modifying it to include traceability parameters. Throughout this section we compare the results obtained with the additional feature of traceability to those obtained in the milk flow model in Chapter 2. When we consider the value output by the model, we will be looking at the value coming out the end of the supply chain which is the result of all three stages working together. In assessing the value of traceability we will be analysing the value it contributes across all three stages of the supply chain, regardless of what stage the traceability parameters and effects are directly influencing.

In comparing the simulations we will obtain a value for certain levels of traceability, given that the desired affect is achieved. If traceability is perfect, then the location of any unit of product is always known perfectly at any point in time. This means in the event of product rejection, only product likely to be contaminated is rejected. For our simulations in this section, if we invest in traceability, it is perfect traceability. If we are able to predict rejection in later stages based on tests done when the milk is collected from the farm, then we can shift the rejection on arrival at the factory from primary to secondary. We will estimate how much such a preemptive rejection is worth based on how much it reduces loss via primary rejections.

Product loss due to contamination could occur at either of the factory reception or processing stages, or both. The loss could also be due to contamination occurring at any stage prior to detection. If a contamination can be identified earlier in the supply chain, product loss can be reduced. If we increase surveillance and traceability, we will increase the rate of secondary rejection but in the process, the number of primary rejections will be reduced. In this section we explore the impact of using traceability to reduce loss at each of these stages.

\subsubsection{Parameter Values for Dairy in New Zealand}

In order to explore the impact of traceability, we use the model developed in Section 3.4 to simulate a variety of different scenarios. To ensure the simulations reflect reality, we base the parameter values for the model on data from Fonterra, the largest dairy company in the New Zealand industry. Some parameter values, such as collection frequency, will remain the same throughout 
the simulations, however some will be varied in order to explore the impact of traceability in different scenarios. The parameters are all summarised in Table 3.2.

\begin{tabular}{|c|c|c|}
\hline Parameter & Description & Initial values \\
\hline V & Amount of milk collected from an on-farm vat & $\$ 3050$ \\
\hline$\Phi$ & Frequency of collection a by tanker & 11,970 \\
\hline $\mathcal{X}$ & Frequency of delivery to factory & 3990 \\
\hline$\Psi$ & Frequency with which milk enters processing & 343 \\
\hline$\Omega$ & Frequency of production & 346 \\
\hline$E_{T}$ & Costs of testing milk at collection site & $\$ 1.90$ \\
\hline$L_{T}$ & Cost of tracing collected milk & 0 \\
\hline$E_{F}$ & Cost of testing milk upon delivery & $\$ 1.90$ \\
\hline$L_{F}$ & Cost of tracing accepted milk & 0 \\
\hline$D_{F}$ & Cost of disposing of unwanted milk & 0 \\
\hline$E_{P}$ & Cost of testing prior to processing & $\$ 1.90$ \\
\hline$L_{P}$ & Cost of tracing milk accepted for processing & 0 \\
\hline$D_{P}$ & Cost of disposing of unwanted milk at factory level & 0 \\
\hline$\alpha$ & Probability of acceptance by tanker & 0.9999 \\
\hline$\beta$ & Probability of passing tests upon arrival at factory & 0.99 \\
\hline$\gamma$ & Probability of passing pre-processing tests & 0.99999 \\
\hline$\eta$ & Probability there is no secondary rejection & 0.9999 \\
\hline$\theta$ & Probability of no secondary rejection & 0.9899 \\
\hline$\varsigma$ & Type 1 conditional 2nd tank acceptance probability & 0.495 \\
\hline$\varpi$ & Secondary conditional 2nd tank acceptance probability & 0.49995 \\
\hline$\varrho$ & Partial silo acceptance probability & 0.99999 \\
\hline$\lambda$ & Factory Traceability Factor & 1 \\
\hline$\varepsilon$ & Silo mixing error & $\$ 42,820.80$ \\
\hline$\ell$ & Processing traceability factor & 1 \\
\hline$C_{T}$ & Capacity of one milk tanker & $\$ 10,705.20$ \\
\hline$N_{T}$ & Capacity of tanker stage & $\$ 5,620,230$ \\
\hline$C_{F}$ & Capacity of one reception silo & $\$ 89.000$ \\
\hline$N_{F}$ & Capacity of factory reception stage & $\$ 8,811,100$ \\
\hline$Q$ & Process exit amount & $\$ 187,230$ \\
\hline$N_{P}$ & Capacity of Processing stage & $\$ 18,537,830$ \\
\hline$\Delta t$ & Time step (days) & 0.00008102 \\
\hline
\end{tabular}

Table 3.2: Parameter values in the perfect traceability scenario. All frequencies are the average number of occurrences per day.

\section{Milk Tanker Parameters}

The number of dairy herds in New Zealand has been steadily declining since 1980, but has recently begun to increase again slightly, beginning in the 2007/08 season. The number of herds increased by forty three in the 2014/15 
season to 11970 (LIC \& DairyNZ, 2015). The capacity of an on-farm silo is based on each cow producing twenty five litres of milk per day at the peak of the season. Fonterra currently requires their suppliers to have a minimum of four hundred litres available at each collection (Fonterra Co-operative Group, 2014b). We estimate an average collection amount per day, during the main season, based on herd size and cow output data. The details of this are given in Table 2.3.

Fonterra's tanker fleet operates 24 hours a day, with a ten to twelve hour day shift involving three to six runs per tanker in Darfield, one of Fonterra's key factories. There is a one to two hour turnover before the night shift starts with a similar pattern to the day shift (McColl, 2011). We assume that a similar structure applies to tanker operation throughout New Zealand. Each run involves delivering to the factory once every run, a tanker completes an average of 7.6 deliveries per day.

The price Fonterra pays farmers in $\$$ per kilogram of milk solids (kg MS), is calculated based on the global dairy trade prices for whole milk powder, skim milk powder, anhydrous milk fat, butter, and buttermilk powder. Because these prices are in US dollars, the exchange rate must be taken into account before Fonterra subtracts the lactose cost and the cash and capital cost (NZX Agri et al., 2014). The farm gate milk price for the 2014/2015 season was $\$ 4.40$ (Fonterra Co-operative Group, 2015a). This price takes into account fixed costs such as transport and manufacturing as well as allowing for appropriate returns on investment (Fonterra Co-operative Group, 2017a).

$V$ is the average amount of milk collected from a farm vat. Based on the information in Table 2.3, during the main milking season each farm is producing an average of $693 \mathrm{~kg}$ MS per day, during the peak months of the year this jumps to $808 \mathrm{~kg}$ MS. Using a price of $\$ 4.40$ per $\mathrm{kg}$ MS we can estimate $V$, the average value of milk produced by and collected from each farm per day. $V=\$ 4.40 \times 693=\$ 3049.20$.

$\Phi$ is the frequency of collection attempts. We can estimate this as the number of on farm vats that are collected from each day. There were 11,970 herds supplying Fonterra in the 2014/2015 season (LIC \& DairyNZ, 2015). If we assume all herds are being collected from every day during the main production season then $\Phi=11,970$. 
$E_{T}$ is the estimated cost of testing milk when it is collected by the tanker. Livestock improvement incorporation (LIC) charges a rate of $\$ 1.99$ per animal to conduct a suite of tests for milk quality (LIC New Zealand, 2012). We base our test price off this rate, reducing the value slightly to account for the amount of testing being requested or conducted by Fonterra we estimate $E_{T}=\$ 1.90$.

$L_{T}$ is the cost associated with traceability implemented at the tanker stage. While we have included this term in our model, in the following simulations we are trying to estimate the overall value of traceability, from which this would be a direct subtraction. It is simpler in this case to estimate the total value, to which the cost of the whole traceability system can be compared. Therefore we set $L_{T}=0$.

$\alpha$ is the probability that the milk passes all testing and is accepted by the tanker. Information obtained though discussion with Fonterra staff suggests we set $\alpha=0.9999$ (T. Kirk, oral communication, November 2015). This translates to an everyday rejection rate of $0.01 \%$, or approximately 1.2 vats each day.

$C_{T}$ is defined as the average capacity of one milk tanker. Each milk tanker, truck and trailer unit can hold 28,800 L of milk (Dooley et al., 2005), this is equivalent to $2433 \mathrm{~kg} \mathrm{MS}$, therefore $C_{T}=\$ 10705.20$.

$N_{T}$ is the capacity of the entire fleet of tankers. Fonterra operates a fleet of 525 tankers (Mason, 2014) so $N_{T}=\$ 10705.20 \times 525=\$ 5620230$.

$\mathcal{X}$ is the frequency with which milk tankers deliver milk to the factory. If each tanker collects from an average of threee farms during each run, then we need $\frac{11970}{3}=3990$ tanker runs every day. Given there is one delivery at the end of each run $\mathcal{X}=3990$.

\section{Factory Reception Parameters}

Fonterra has the capacity to process about seventy million litres of milk per day during the peak season (Fonterra, 2010). Milk reception silos range in size from 225,000 to 500,000 litres. 
$E_{F}$ is the cost of testing milk as it arrives at the factory. Similar to $E_{T}$ in Section 3.5.1, we estimate $E_{F}=\$ 1.90$.

$L_{F}$ is the cost associated with traceability implemented at the factory reception stage. For the reasons outlined in Section 3.5.1 regarding $L_{T}$ we set $L_{F}=0$.

$\beta$ is the probability that a tanker load is accepted by factory. This is the stage with the greatest rate of rejection. Based on conversations with Fonterra personnel, an average of $1 \%$ of milk is discarded upon arrival at the factory (T. Kirk, oral communication, November 2015). This gives us $\beta=0.99$.

$\varsigma$ is the probability the second tank of a tanker will be accepted, given that the first tank was rejected. Because each tanker visits an average of three farms, there is about a $50 \%$ chance that the contaminated load spans both tanks, as explained in Section 3.4.5. Taking this into account along with the possibility there is a second unrelated contamination we can estimate $\varsigma=0.5 \beta=0.495$

$\lambda$ is the traceability factor. It represents whether we have sufficient traceability to distinguish between tanks on a tanker or not. In this scenario we can distinguish between tanks, therefore $\lambda=1$.

$\eta$ is the probability that a tank load of milk is not rejected by a secondary rejection upon delivery to the factory. This is essentially a delayed rejection of farm vats and the rejection rate reflects that. We use $\eta=\alpha=0.9999$

$\varpi$ represents the conditional probability that the second tank is accepted given the first tank of the tanker is rejected by a secondary rejection. Because we have perfect traceability, the second tank will only be rejected if milk from contaminated farm vat was loaded into both tanks. As outlined in Section 3.4.5 with perfect traceability the second tank can be accepted in $50 \%$ of situations. So we let $\varpi=0.5 \eta=0.49995$.

$D_{F}$ is the cost associated with disposing of rejected milk at the factory reception level. Most rejected milk can be used as calf feed or sprayed on crops as fertiliser. Fonterra does contract tankers from outside their 
own fleet to transport this rejected milk, but the associated costs can generally be recouped in the price paid for this rejected product. Because of this we set $D_{F}=0$.

$C_{F}$ Each processing site has multiple reception silos, as mentioned above, a typical paediatric site has silos of 225,000 litre capacity. This equates to $20,228 \mathrm{~kg}$ MS therefore $C_{F}=\$ 89,000$.

$N_{F}$ The typical paediatric processing site has three reception silos, with thirty three processing sites around the country this gives a total capacity of $33 \times 3 C_{F}=2,002,572 \mathrm{~kg}$ MS leading to $N_{F}=\$ 8,811,100$.

$\Psi$ is the rate at which milk moves into the processing stage from the reception silos. Each separator is capable of separating 33,000 litres of raw milk each hour. This gives $33 \times 3 \times 33,000 \times 24=78,408$, 000 litres per day, which is equivalent to $6,923,463 \mathrm{~kg}$ MS per day. Dividing this by the capacity of one reception silo $C_{F}$ gives us $\Psi=343$

\section{Processing Parameters}

Aside from small quantities of on farm sales, the first steps in production required for all dairy products produced in New Zealand are separation, standardisation, and pasteurisation (Hudson et al., 2003; Western, 2011). In this model we will focus on the first two of these stages, separation and standardisation. Typically a factory has a bank of several separators which feed into several silos for cream and skim milk. Each separator bowl has a volume of fifty litres and is capable of separating 33,000 litres every hour (Centrico, 1994).

$E_{P}$ is the cost of testing conducted upon delivery to the factory. Assuming the range of tests conducted pre-processing is similar to those conducted before acceptance into the factory, we set $E_{P}=\$ 1.90$

$L_{P}$ is the cost associated with traceability implemented at the processing stage. Because any impact of traceability implemented at this stage would not be seen until later stages that are not currently modelled, and we are assessing the value contributed by traceability, we set $L_{P}=0$. 
$\gamma$ is the rate of rejection before entry into the processing stage. This was also discussed with staff at Fonterra, and has the lowest rejection rate of the three stages (T. Kirk, oral communication, November 2015). Once the milk is inside the factory the environment is much more controlled, the potential for contamination or spoilage is greatly reduced. We set the chance of rejection at $0.001 \%$, implying $\gamma=0.99999$.

$\theta$ is the probability that a factory reception silo does not have any of its contents rejected by a secondary rejection. This probability reflects the value of $\beta$ and that of $\alpha$, the probabilities that material is not rejected instantly at the tanker and factory reception stages. Therefore we have $\theta=0.9999 \times 0.99=0.9899$.

$\varrho$ is the probability that product leftover following a partial rejection is accepted following retesting. A partial acceptance can only take place in a secondary rejection situation. Therefore in a perfect traceability scenario $\varrho=\gamma=0.99999$.

$\ell$ determines what portion of a contaminated silo can potentially be accepted based on the level of traceability employed in the factory reception stage. Because we have perfect traceability in this scenario $\ell=1$.

$\varepsilon$ represents the mixing of milk in the reception silo, and the amount either side of a contamination that must be rejected along with the contaminated tank volume. This is where we bring in a bit of fuzzy traceability (Skoglund \& Dejmek, 2007). Because milk is liquid this value is relatively large, we set this at $\varepsilon=4 C_{T}=\$ 42820.80$.

$D_{P}$ is the cost of disposing of product when it it rejected at the processing stage. As discussed in reference to $D_{F}$, disposal cost is negligible so we can set $D_{P}=0$.

$N_{P}$ is the total capacity of the processing stage. The typical paediatric processing site has a bank of Three separators feeding into two cream silos, three skim milk silos and two excess silos. This gives a total capacity of $3 \times 50+2 \times 95000+3 \times 350000+2 \times 90000=1,420,150$ litres (Fonterra Co-operative Group, 2016a), equivalent to $127,670 \mathrm{~kg}$ 
MS. As there are thirty three processing sites around the country $N_{P}=$ $127,670 \times 4.40 \times 33=\$ 18,537,830$.

$Q$ is the value of milk leaving the processing stage each time step. In reality there is continuous flow through this stage we have some freedom in the value we choose for $\mathrm{Q}$. We really just require that $\mathrm{Q}$ is large enough to keep up with Fonterra's rate of production. For our simulations we use the sum of the silo capacity in $N_{P}$ divided by $33 \times 3=99$, the number of silos feeding in. This is equivalent to $Q=\$ 187,230$.

$\Omega$ is the rate at which product leaves the initial processing stage and moves on to further processing. To allow for enough product flow each day we require that $\Omega \times Q$ is greater than $\$ 27,689,200$, the value of milk produced by Fonterra every day in peak season (Fonterra, 2010). We use $\Omega=346$ for consistency with the model in the previous chapter.

\section{Time Step}

Using Equation 3.17, we can calculate the maximum allowable value for the time step as

$$
\begin{aligned}
\Delta t & \leq \frac{1}{\max \{11970,3990,(343+346)\}} \\
\Longrightarrow t & \leq \frac{1}{11970}=0.00008354 \text { days or } 7.218 \text { seconds }
\end{aligned}
$$

This suggests we use a time step size of 7 seconds, giving $\Delta t=0.00008102$. This will allow a tanker to be delivering or collecting milk almost every time step.

\subsubsection{Reducing Factory Reception Rejection}

The first point in the supply chain where the effect of traceability may be seen is the factory reception stage. Initially we will analyse the effect of traceability on total output when it effects this stage alone. Figure 3.8 shows the 24 hour production output value in a scenario where $\beta=0.75, \lambda=0$, and $\eta=1$. In this scenario $25 \%$ of product needs to be rejected upon arrival at the factory, due to some previously undetected contamination or issue with 
the milk. The difference in production when we transfer this rejection from primary to secondary via traceability (where $\lambda=1, \beta=1$, and $\eta=0.75$ ) is also shown. In this simulation we eliminate primary rejection upon arrival at the factory reception stage, by increasing the secondary rejection rate (due to tests conducted prior to collection by the tanker) to $25 \%$. That is, through increased traceability we allow for more contaminated product to be detected before testing, resulting in a higher secondary rejection rate, but a lower primary rejection rate, meaning less product is discarded overall. This secondary rejection ability comes from implementing good traceability protocols when the tanker collects the milk from the farm. This results in us retaining $\$ 1.8$ million of product per day that would otherwise be disposed of. In each of these simulations the acceptance rate of milk from farm vats is held constant at $\alpha=0.9999$.

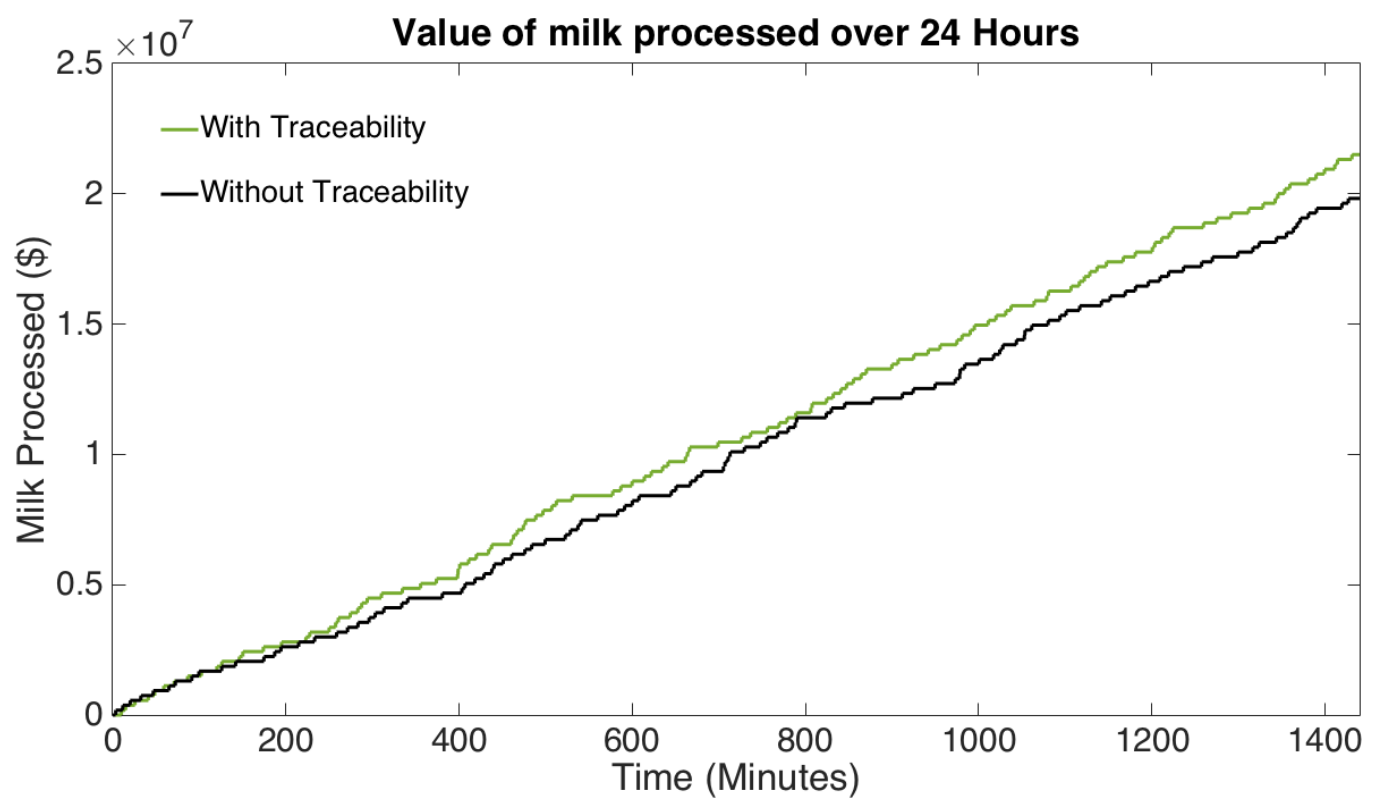

Figure 3.8: Simulations of milk produced over a 24 hour period with a $25 \%$ rejection rate of product entering the factory reception. One simulation of each scenario is shown.

Table 3.3 summarises the results of five hundred simulations, transferring primary rejection to secondary, for a variety of $\beta$ values. The new minimum $\beta$ is the rate of primary acceptance required to ensure a positive traceability impact. For example, if we start with a primary acceptance rate of $\beta=0.85$, when we introduce traceability to the effect that the secondary acceptance probability is reduced to $\eta=0.85$, the primary acceptance probability must 
be increased to at least $\beta=0.96$ to have a positive impact on output value. The potential gain if we are able to eliminate primary rejection altogether is given in the last two columns of Table 3.3 as a total dollar value and as a percentage increase from the no traceability scenario. Each value given is the average total 24 hour output over five hundred simulation runs. For all of the simulations we hold the collection acceptance rate constant at $\alpha=0.9999$.

\begin{tabular}{|c|c|c|r|c|}
\hline \multicolumn{2}{|c|}{ Traceability Affecting Factory Reception Acceptance Only } \\
\hline \multicolumn{2}{|c|}{ No traceability } & \multicolumn{3}{|c|}{ With traceability } \\
\hline $\begin{array}{c}\text { Initial } \\
\beta\end{array}$ & $\begin{array}{c}24 \text { Hour } \\
\text { Output Value }\end{array}$ & $\begin{array}{c}\text { Minimum } \\
\text { New } \beta\end{array}$ & \multicolumn{2}{c|}{ Potential Gain } \\
24 Hour Value $\%$ Gain \\
\hline 0.75 & $\$ 20,144,014$ & 0.93 & $\$ 1,902,164$ & $9.4 \%$ \\
\hline 0.80 & $\$ 21,352,034$ & 0.94 & $\$ 1,561,426$ & $7.3 \%$ \\
\hline 0.85 & $\$ 22,592,592$ & 0.96 & $\$ 1,377,442$ & $6.1 \%$ \\
\hline 0.90 & $\$ 23,933,756$ & 0.97 & $\$ 908,446$ & $3.8 \%$ \\
\hline 0.95 & $\$ 25,177,680$ & 0.99 & $\$ 576,708$ & $2.3 \%$ \\
\hline 0.99 & $\$ 26,181,496$ & 1.00 & $\$ 200,090$ & $0.8 \%$ \\
\hline
\end{tabular}

Table 3.3: Simulation outcomes for various rejection and traceability scenarios, where only the probability of product being accepted at the factory reception $\beta$ is improved by the traceability system.

We can see that as the initial primary acceptance rate increases, the potential for improvement is reduced. Even so the overall output value still increases. The improvement in primary acceptance rates is also reduced as the initial primary acceptance rate increases. The last row of Table 3.3 shows the outcome if we are able to eliminate all primary rejection. The total output value becomes $\$ 26,181,496+\$ 200,090=\$ 26,381,586$. Given that, while paying for testing and current traceability standards, Fonterra can produce over $\$ 26,000,000$ of product per day (Fonterra, 2010), this model produces the output we would expect.

Figure 3.9 summarises the results from the five hundred simulation runs with box plots for each primary rejection scenario, and for each scenario where all of the rejection is managed through traceability and secondary rejections. All of the plots are fairly symmetrical, they each have quite a large spread overall but the interquartile ranges are relatively small. In each case there is overlap between the value processed in the with and without traceability scenarios, 
but as the acceptance rate decreases, and the potential for improvement increases, the plots become more distinct. If we are able to mostly remove primary rejection, without increasing secondary rejection to the same rate, we can have scenarios with no overlap at all. For example in a scenario where we are rejecting $20 \%$ of product as it arrives at the factory via primary rejection, if we are able to eliminate this primary rejection by improving traceability and product identification such that $10 \%$ of product is rejected in secondary rejections, we will improve total production value every day. Even if there is some overlap between scenarios, remembering that these are simulations of one day's production, the $95 \%$ confidence interval for the mean in each traceability versus no traceability comparison is distinct, meaning on average traceability is an improvement in each case.

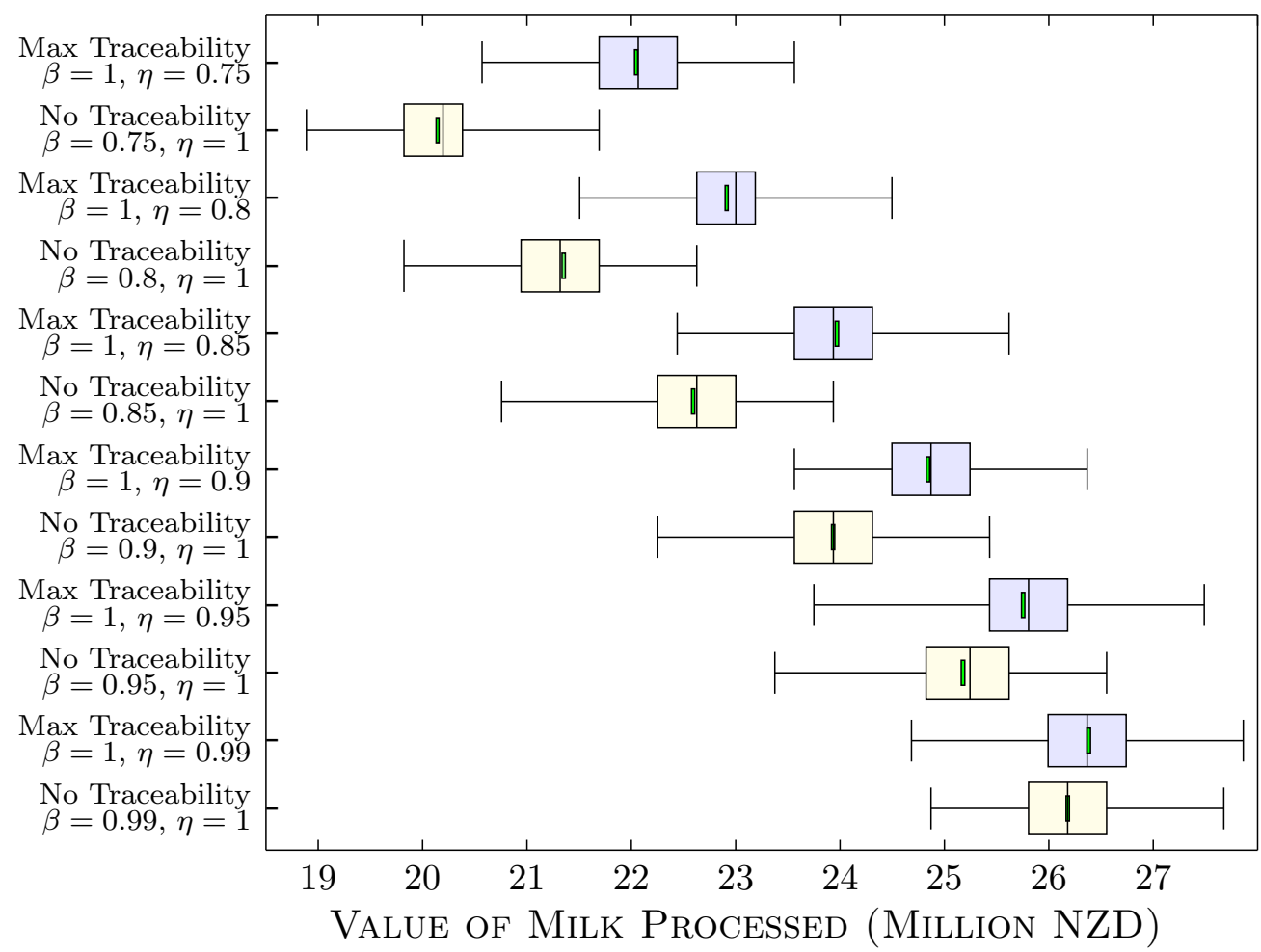

Figure 3.9: Box plots for the value of milk processed over a 24 hour period in various scenarios, with and without traceability, over five hunderd simulations. The confidence interval of the mean is also shown as a small green box within each plot. 


\subsubsection{Reducing Product Loss at Processing Entry}

When milk enters the processing stage it can be rejected via either primary or secondary rejection. The earlier in the supply chain a contamination is detected, the less product that is potentially contaminated and necessarily disposed of. With a good traceability system, even if the contamination is not known until the product is ready to enter the processing stage, we can identify the original contaminated product and any product that contamination may have spread too. This allows us to dispose of only product likely to be contaminated, thus reducing losses. For the scenarios simulated in this section, information relevant to traceability is collected at the tanker and factory reception stages, but this information is only applied as material enters the processing stage. We run simulations for various values of $\gamma$, the rate of primary rejections at the point of processing entry. We compare these results to scenarios including traceability to investigate how much of an impact it will have on loss reduction and thus, overall production. Figure 3.10 shows one simulation each of before and after the implementation of traceability effects, using an acceptance rate of $\gamma=0.75$ and $\eta=1$. In this scenario, $25 \%$ of product entering the processing stage needs to be rejected, due to some previously undetected contamination. If we can identify more of this product for secondary rejection, as in the simulation with traceability, where $\gamma=1$ and $\eta=0.75$, less will need to be rejected via primary rejection, meaning we reduce losses overall. The potential value of traceability in this scenario, is $\$ 3,600,000$. Table 3.4 shows the simulation results for various rejection rates $\gamma$. Each value given is the average 24 hour production value over 500 simulations. The outcome with no traceability, is given along with the potential gain if we are able to use traceability to eliminate primary rejections. The minimum primary acceptance rate needed to make an output improvement when we increase secondary rejection rates (the minimum new $\gamma)$ is also given. Again the biggest potential gain is seen with the smallest acceptance rate, as this is logically where we will have the most room to improve.

Comparing the implementation of traceability at the factory reception stage with the processing entry stage, overall we see more potential value retention. 


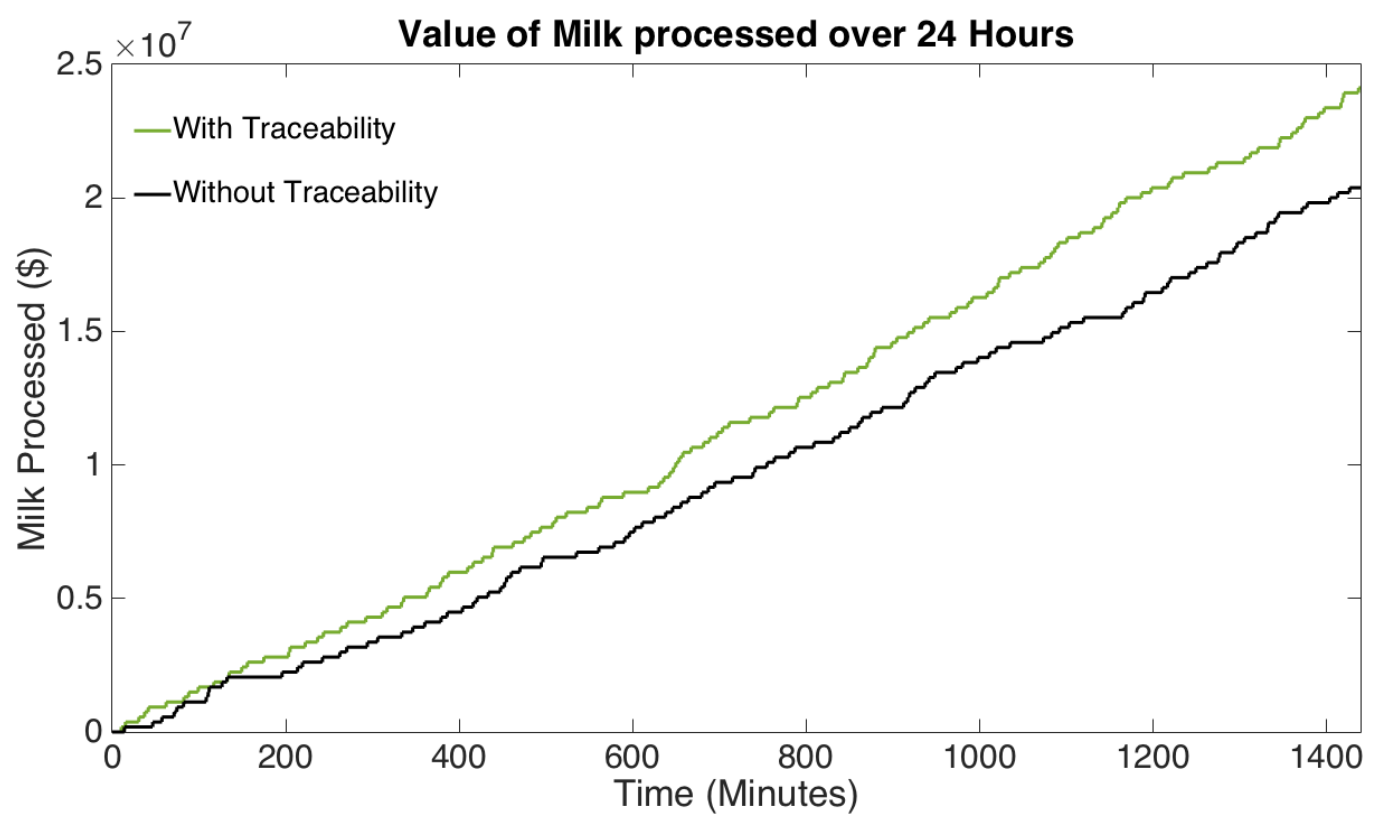

Figure 3.10: A single simulation of milk produced over a 24 hour period, with a $25 \%$ rejection rate of product entering the processing stage.

As the initial acceptance rate increases, the gap between these values closes. The new value for the primary acceptance rate after the implementation of traceability also does not need to be as high in the processing entry in order to see an improvement.

Figure 3.11 shows box plots summarising the results from five hundred simulation runs for each of the primary rejection scenarios given in Table 3.4, along with plots for the scenarios where all primary rejection is eliminated via traceability and secondary rejection. Again most of the plots are fairly symmetrical. The plots for scenarios with traceability get more distinct from the no traceability plots as rejection rates increase in this stage, particularly compared with the plots in Figure 3.9. In fact the overall production value is consistently better in a scenario where we are rejecting $25 \%$ of product in secondary rejections $(\theta=0.75)$, with no primary rejection, than in the scenario where only $15 \%$ of product is rejected in primary rejections $(\gamma=0.85)$ with $\theta=1$. The difference is still quite small with larger acceptance rates, however the $95 \%$ confidence interval for the mean is still distinct in each case. 


\begin{tabular}{|c|c|c|r|r|}
\hline \multicolumn{3}{|c|}{ Traceability Affecting Processing Entry Acceptance Only } \\
\hline \multicolumn{2}{|c|}{ No traceability } & \multicolumn{3}{|c|}{ With traceability } \\
\hline Initial & 24 Hour & Minimum & \multicolumn{2}{c|}{ Potential Gain } \\
$\gamma$ & Output Value & New $\gamma$ & 24 Hour Value $\%$ Gain \\
\hline 0.75 & $\$ 21,567,212$ & 0.86 & $\$ 2,575,236$ & $11.9 \%$ \\
\hline 0.80 & $\$ 22,568,772$ & 0.9 & $\$ 2,012,378$ & $8.9 \%$ \\
\hline 0.85 & $\$ 23,600,896$ & 0.93 & $\$ 1,435,786$ & $6.1 \%$ \\
\hline 0.90 & $\$ 24,516,822$ & 0.95 & $\$ 979,506$ & $4.0 \%$ \\
\hline 0.95 & $\$ 25,426,016$ & 0.98 & $\$ 566,984$ & $2.2 \%$ \\
\hline 0.99 & $\$ 26,069,670$ & 1.00 & $\$ 126,786$ & $0.5 \%$ \\
\hline
\end{tabular}

Table 3.4: Simulation results (average of five hundred simulation runs) for scenarios where the traceability system only affects product acceptance rates at the entry to the processing stage.

\subsubsection{Reducing Product Loss Throughout the Supply Chain}

As mentioned in the previous sections, the earlier a contamination is detected, the more it can be contained and losses limited. In this section, we allow that some contaminations may still take time to show up, and thus not be detected until the processing entry stage, but we also allow that other contamination will be detected earlier, and thus be able to be dealt with earlier. For simplicity we set the initial primary rejection rates $\beta$ and $\gamma$ equal to each other in the following simulations. We then investigate the impact that introducing traceability through secondary rejection can have, when applied at both the factory reception and processing stages.

Figure 3.12 shows a pair of simulations where the rejection rate of $25 \%$ is affecting both the factory reception and processing stages. One simulation without traceability and one simulation with traceability effects is shown, over a 24 hour production period. In the scenario without traceability, $\beta=$ $\gamma=0.75$. In this particular set of simulations including traceability increases total output by $\$ 4,960,000$. Table 3.5 shows the potential value of traceability over the whole model for various initial primary rejection rates $\beta$ and $\gamma$. In each case the value given is the average of five hundred simulation results. The minimum primary acceptance rate required for traceability to have a positive effect (the minimum new $\beta=\gamma$ ) is given, along with the potential value of loss reduction if primary rejection is able to be eliminated altogether. 


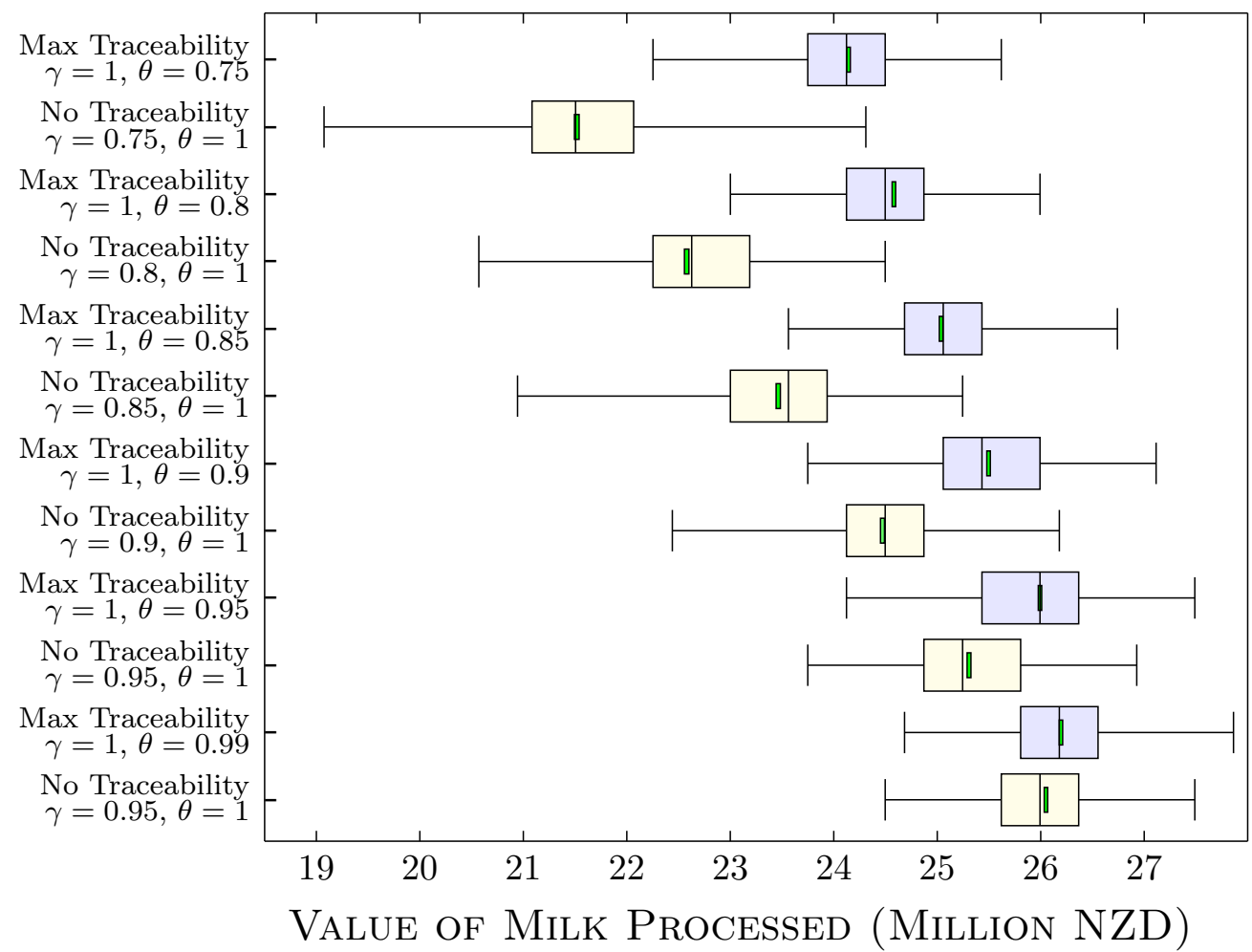

Figure 3.11: Box plots for five hundred simulations of each scenario given in Table 3.4. The $95 \%$ confidence interval for the mean is also shown as a green small box within each plot.

Each value shown in Table 3.5 is the total value of milk passing through all three stages and moving out to the next stage over a 24 hour period.

\begin{tabular}{|c|c|c|c|c|}
\hline \multicolumn{3}{|c|}{ Factory Reception and Processing Entry Implementation } \\
\hline \multicolumn{2}{|c|}{ No traceability } & \multicolumn{3}{|c|}{ With traceability } \\
\hline \multirow{2}{*}{$\begin{array}{c}\text { Initial } \\
\beta \text { and } \gamma\end{array}$} & 24 Hour & Minimum & \multicolumn{2}{c|}{ Potential Gain } \\
\hline 0.75 & $\$ 15,969,426$ & 0.88 & $\$ 4,322,318$ & $27.1 \%$ \\
\hline 0.8 & $\$ 17,924,698$ & 0.91 & $\$ 3,603,116$ & $20.1 \%$ \\
\hline 0.85 & $\$ 19,980,202$ & 0.94 & $\$ 2,805,000$ & $14 \%$ \\
\hline 0.9 & $\$ 22,052,910$ & 0.96 & $\$ 2,019,974$ & $9.2 \%$ \\
\hline 0.95 & $\$ 24,299,154$ & 0.98 & $\$ 1,037,102$ & $4.3 \%$ \\
\hline 0.99 & $\$ 26,091,175$ & 1 & $\$ 1,778,3705$ & $0.7 \%$ \\
\hline
\end{tabular}

Table 3.5: Average simulation results over five hundred runs for rejection and traceability scenarios allowing rejection upon entry to both the factory reception stage and the processing stage. 


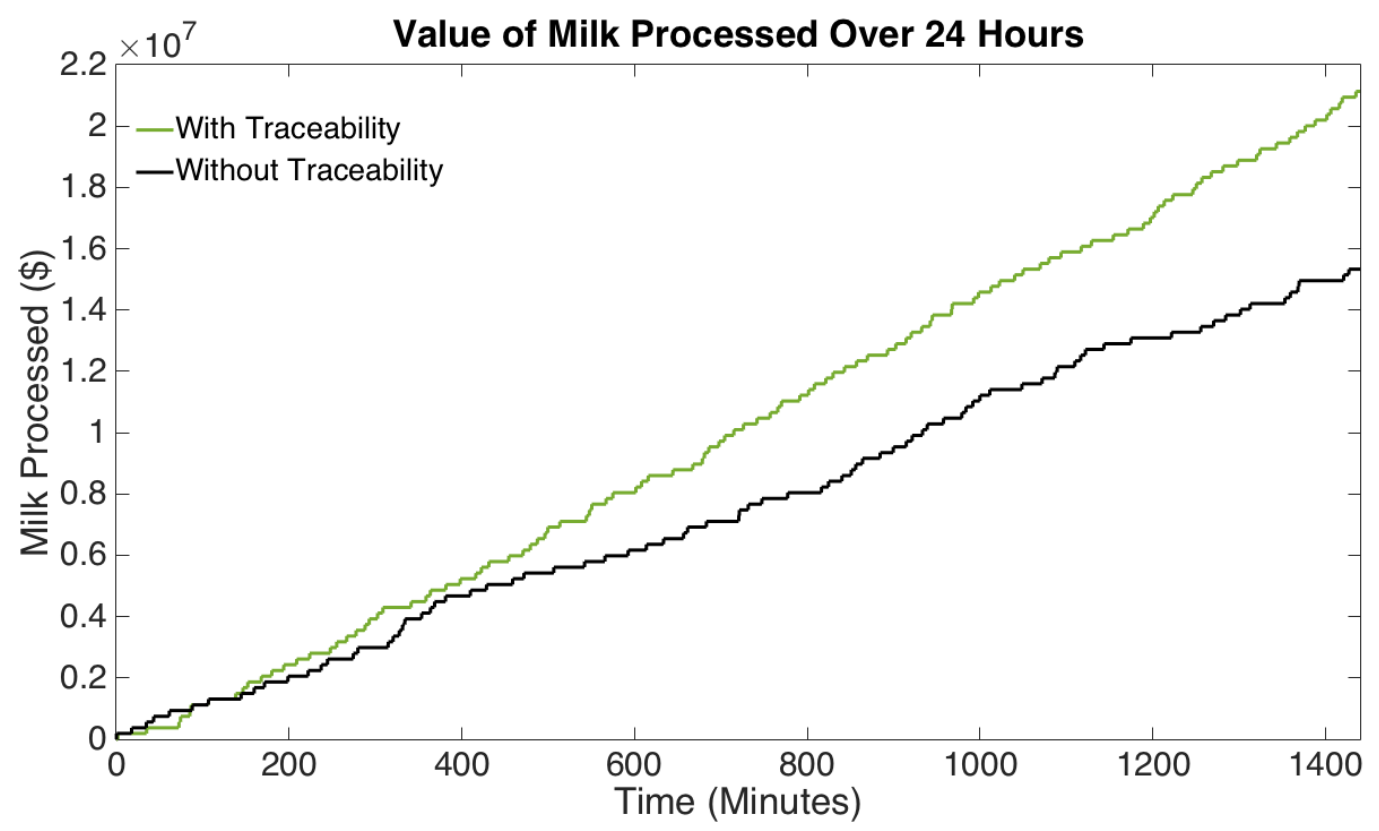

Figure 3.12: A simulation of milk produced over a 24 hour period with a $25 \%$ rejection rate of product entering the factory reception and processing stages.

While we see large improvements in production value with the introduction of traceability effects in cases where the rejection rate would have been high, such rejection rates are not typical of everyday dairy production. Milk tanker deliveries are generally accepted $99 \%$ of the time, while processing entry has a higher acceptance rate of $99.999 \%$. The traceability system that is used needs to react to contamination scares and minimise their impact, while not influencing day to day production negatively. As seen in the simulation results above there is potential for even day to day production to be improved through traceability.

We see also a larger improvement due to traceability when we apply it across multiple stages of the supply chain. As shown in Table 3.5 the effect of traceability applied at both the factory reception and processing stages is greater than the sum of their effects individually. The potential for improvement still drops off quite steeply as the initial acceptance rate increases, though this is to be expected. Figure 3.13 shows box plots for each row in Table 3.5. The larger potential improvement with traceability is noticeable even for the $95 \%$ acceptance rate scenarios. 


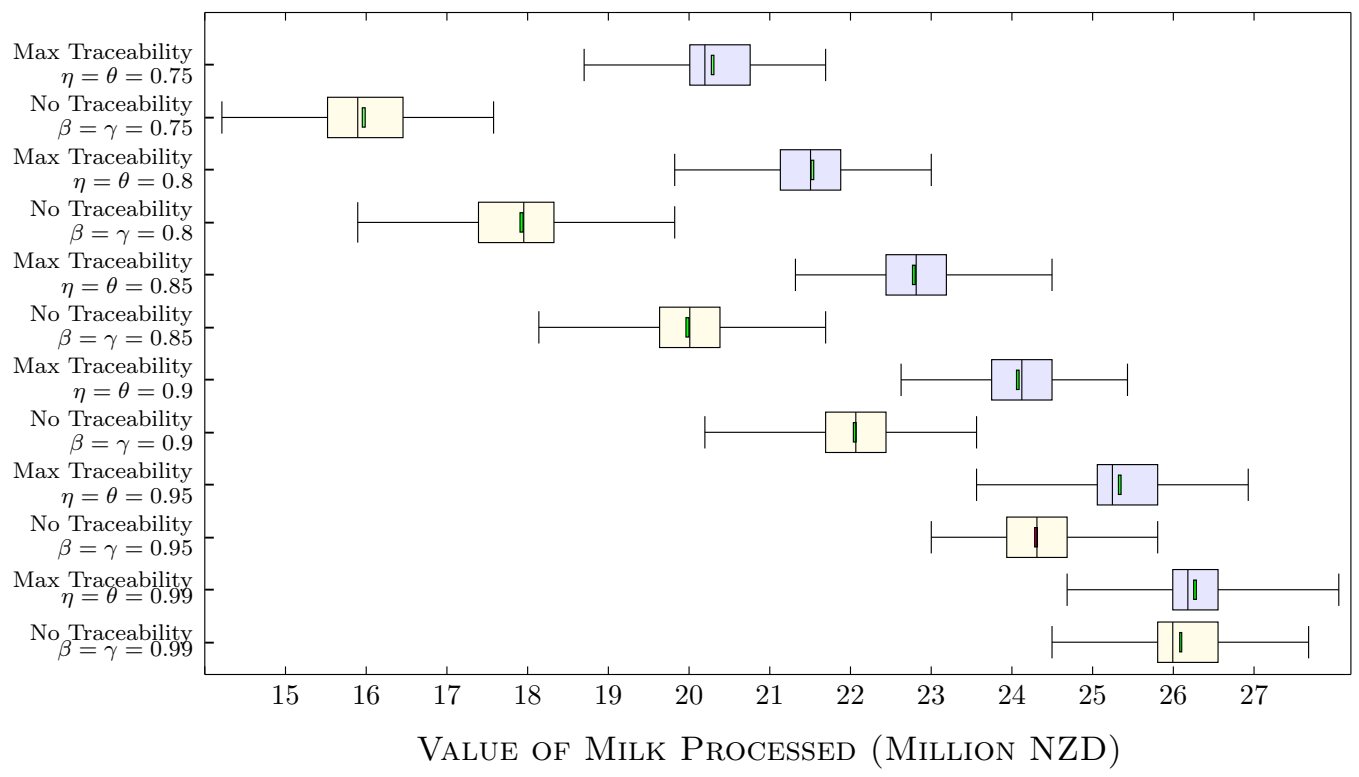

Figure 3.13: Box plots produced after five hundred simulations for the rejection scenarios given in Table 3.5. Both traceability and non-traceability scenarios are shown. The small green box within each plot shows the $95 \%$ confidence interval for the mean.

\subsection{Conclusion}

We have extended the model developed in Chapter 2 to include traceability effects. Using this model, we investigated the impact of traceability in several different scenarios. We have shown there is significant value to be gained when we allow increased secondary rejection via traceability if this means we can reduce primary rejection rates. Separately, traceability has a larger impact when implemented at tanker collection, resulting in reduced primary rejections at the factory reception stage. The probability of primary or secondary rejection at the entry to processing is individually less important. The largest impact, however, is seen when traceability effects are applied across all the stages of the model.

The model we have developed is a useful tool for theoretically assessing the value of traceability in the early stages of the supply chain. It is still just a model however and limited by the information that was accessible during development. We model all of the tankers as one value pool, similarly the factory reception silos and processing sites, which may limit accuracy to some extent. This model also does not account for inter-site transfers that 
may happen between the factory reception and processing stages. We only model the first few stages in the dairy supply chain in this thesis, the network of product flow becomes more complicated as we progress through the supply chain and more ingredients and products begin to interact. The costs associated with testing and traceability are kept constant in the simulation presented. Some analysis was done on the impacts of different costs, however anything realistic did not have any significant impact on Fonterra's overall production. This may be worth further investigation in the case of applying the model to the other industries or smaller companies, but was not helpful in achieving the aims of this chapter. We briefly mentioned the possibility of using a continuous time model, it would be interesting to compare the results of similar simulations using each type of model. This would also make choosing the time step size unnecessary. This model also does not include the costs of lost "goodwill" and reputation, focusing solely on the cost of product loss and reducing this. If we were to include reputation effects, the value of traceability may in fact be higher.

This chapter fills a gap in traceability literature by using a stochastic model to investigate the value of traceability to a supply chain. There is still plenty of scope for future research into the effects of traceability throughout the supply chain, possibly extending the model to follow products to completion, retail, and potentially all the way to the customer. Increasing the resolution of the model to follow individual product locations more closely, for example modelling each tanker individually, is another direction future research could take. This model serves as a good starting point to further investigate traceability in dairy, extending this research in either direction. 


\section{Chapter 4}

\section{Recalls, Storms and Import Bans: The Impact of Shocks on Share Prices in The New Zealand Dairy Industry}

\subsection{Introduction}

Information regarding how the stock market reacts to shocks has many uses. One such use is in influencing decisions around safety and quality control systems (Salin \& Hooker, 2001). Assessing the economic impact of food recalls, for example, necessitates a thorough understanding of the costs incurred for the firms involved. This however requires access to firm-level data that is generally not available (Pozo \& Schroeder, 2015). How the stock market reacts to recalls of varying size, scope, and resolution time has particular importance for designing traceability systems. Such data can inform on the value of maintaining traceability standards. The reason behind the recall, and how different types of recalls influence stock price are also useful for determining what aspects of products companies should seek to control and mitigate most (through traceability or other means). In this chapter we investigate the behaviour of Fonterra's share price returns surrounding various shocks, including recalls, adverse weather events, and global demand shocks. In the remainder of this introduction we discuss previous literature in assess- 
ing the impact of shocks on shares prices in production companies. We follow this with an introduction to the New Zealand dairy industry and how it fits into the stock market. In Section 4.2 we introduce each of the events we will be investigating. These include product recalls, adverse weather events, and events that affect global dairy supply and demand. We are interested in how the stock market might react differently to different types of shocks. In Section 4.3 we discuss the method for conducting this analysis and the data we use. The analysis and results are presented for each group of events in Section 4.4. We briefly discuss the implications of these results as they arise. Finally, we discuss the limitations of this research and our conclusions in Section 4.5.

\subsubsection{Assessing the Impact of Shocks on Share Prices}

In this section we discuss some of the existing literature assessing the impact of shocks on share prices and the methodology they use. Brown \& Warner (1985) state that the most efficient indicator of changes in financial performance, due to an event, is the reaction of stock prices.

Though there are many studies assessing the impact of recalls on consumer demand and shareholder wealth, these primarily consider the automobile and pharmaceutical industries. Krieger \& Chen (2015) analyse the impact of aircraft accidents on their manufacturer's stocks. They find a significant negative impact immediately follows each accident, however whether the stocks continue to fall or recover quickly over the next few weeks shows strong correlation with whether the manufacturer is judged to have a potential link to the incident.

Previous research on consumer food safety preferences has generally occurred at the market level, focussing on reactions to risk for commodity groups, rather than for particular companies. Salin \& Hooker (2001) use a partial event analysis technique to examine the cost of food recalls from the perspective of capital markets. They specifically investigate the firm-specific repercussions of microbiological contamination incidents. They conduct an event study of stock returns to examine the reactions of shareholders. In some cases they saw a drop in returns to shareholders, but found no discernible market reaction in others. The smaller firms appeared to be the 
most affected, they saw a steep drop in market values immediately following a food recall. The methodology used is relatively straight forward, however the simplicity of the models they use and the resulting low $R^{2}$ values may lead to underestimation of the market reaction. Pozo \& Schroeder (2015) investigate how certain characteristics of meat and poultry recalls affect the magnitude of stock price reactions. They initially use an event study to estimate the cumulative average abnormal returns, then assess the relationship between these and relevant recall characteristics. They find that larger recalls have a greater negative impact on stock price, but interestingly, recurrent recalls have a lesser effect. It appears experience in dealing with recalls has a positive impact. Each subsequent recall may also have less individual influence, as the likelihood of recalls is already incorporated into the share price. The volume of media coverage surrounding the recall was also found to have a proportionally negative impact on stock price. Their work highlights the advantage of estimating pooled models, as opposed to cross-sectional. The study is limited to short term price reactions, which means any longterm effects of the recalls may not be seen. A different approach would be needed to measure these effects accurately.

Gao (2011) investigates the stock market response to the 2008 China melamine milk scandal. They use an event approach and a generalised autoregressive conditional heteroskedasticity (GARCH) model to examine asymmetric impacts of the scandal on the level and volatility of dairy company stock prices. Their results suggest that overseas stock markets were more responsive to the contamination announcement compared to those traded in China. These foreign markets were slower to respond when positive information regarding product safety was released. All of the companies experienced a drop in stock prices while waiting for test results. Following verification of contaminated products, those companies involved saw a further drop, while those with no contaminations rebounded to pre-event levels. Despite being the core foreign partner to the first dairy company in China found to have contamination, Fonterra was not included in this analysis. Fonterra's trading structure has changed since this study was done however, becoming more relevant to this style of investigation.

Production delays can also cause a reaction in the share markets, particularly in perishable food supply chains. Hendricks \& Singhal (2003) estimate the 
effect of supply chain glitches on shareholder wealth. Focusing on glitches that result in production or shipment delays, they find significant decreases in shareholder value following glitch announcements, regardless of who is responsible for the glitch. Alves et al. (2015) use structural equation modelling to examine the effect of governance rules on share price and liquidity. They construct a voluntary disclosure index, which they use to analyse indirect relations through voluntary disclosure of information and organisational performance. They conclude that ownership structure directly influences share price and liquidity. Firms with high levels of disclosure tend to have a lower bid-ask spread, while firms with high ownership concentration have less trading, increased bid-ask spread and reduced liquidity.

Hanson et al. (1993) use an input-output model to analyse cost linkages between energy and other sectors. then, using United States Department of Agriculture/Economic Research Service computable general equilibrium model, they to analyse the sectoral effects of a world oil price shock under different scenarios. Their costs analysis suggests there is a negative effect on agricultural sectors when there is an oil price shock. Dairy and livestock in particular experience a decline in output, they do show a rise in price, but a decline in value added and sectoral income is observed alongside this.

Share prices may also be affected by adverse weather conditions. The impact of hurricanes on the stock returns of large American energy companies is measured by Liu et al. (2015). They found very different results depending on how the company in question produced energy. In the case of renewable energy, an increase in cumulative average abnormal stock returns was seen in the days right after the event, before beginning to decrease, where as other energy sources saw a decrease beginning immediately after the event.

\subsubsection{The New Zealand Dairy Industry}

Dairy is an important industry in New Zealand. Dairy production contributes $\$ 7.8$ billion to New Zealand's total gross domestic product (GDP), approximately $3.5 \%$. In terms of exports, it currently accounts for $29 \%$ of export dollars coming into the country (Ballingall \& Pambudi, 2017). Around 97\% of dairy farmers in New Zealand sell their milk through Fonterra Cooperative Group (Jiang \& Sharp, 2014). Any significant financial impact on Fonterra 
will impact the whole country.

Fonterra was formed in 2001, from the merger between Kiwi Cooperative Dairies Limited, the New Zealand Cooperative Dairy Company Limited, and the New Zealand Dairy Board. It is a farmer owned cooperative, all holders of Fonterra Cooperative Group shares (FCG) are dairy farmers supplying Fonterra (Ballingall \& Pambudi, 2017). Farmers can choose to convert shares to freely traded units in the Fonterra Shareholders Fund (FSF), traded on the New Zealand Stock Exchange (NZX) and Australian Stock Exchange (ASX). The price of New Zealand traded FSF units is highly correlated with the price of FCG shares. The FSF units traded on the ASX are not considered in this thesis. Farmers are required to hold at least one share for every kilogram of milk solids supplied (Jiang \& Sharp, 2014). Initially these shares were set at a nominal value, as they had been in the previous cooperatives. Shares were only exchanged vertically, between farmers and Fonterra. Increasing challenges for Fonterra from 2007, together with shares being strictly linked to production, led to farmers being required to sell shares and Fonterra to purchase them under the less than favourable conditions associated with the global financial crisis. These challenges pushed Fonterra to change their capital structure. In 2009 'dry shares' were introduced, allowing farmers to hold shares in excess of their production. This required the unbundling of payment for milk supply from dividend on shares. Next, by allowing farmers to trade shares amongst themselves, the obligation for Fonterra to issue and redeem shares was removed. From December 2012 farmers have been able to trade FCG shares amongst themselves, and convert these shares to FSF units for public trading.

China is consistently the largest importer of New Zealand dairy products. Table 4.1 shows the value exported to specific countries from 2013 to 2016. Years run from 1 July to 30 June (MFAT \& Statistics New Zealand, 2016). Only countries that were in the top five in terms of export value in at least one of these years are shown.

The United States are consistently second in terms of export value, but receive a very different mix of products compared to China. The top dairy exports to China are milk, cream, and concentrated products, followed by butter products. The United States receive mostly casein and caseinate products; these are used in a variety of applications, including but not limited to 


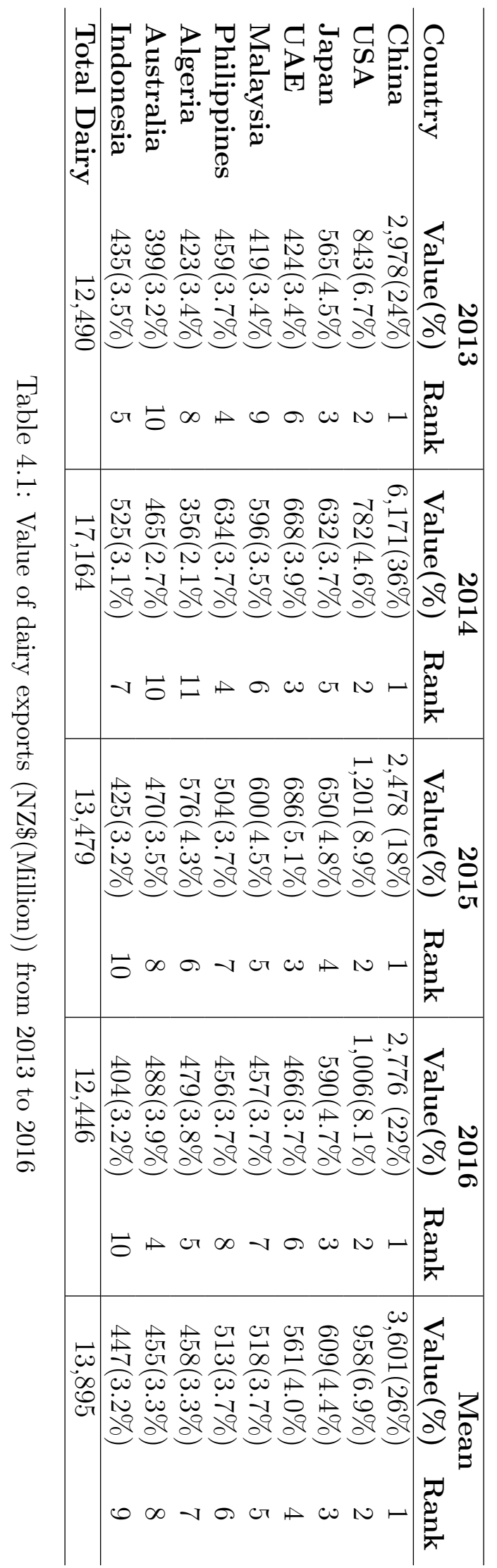


food products. Table 4.2 shows the value exported by product type. The average value exported per year from 2013 to 2016 in Millions of New Zealand dollars, is given for the top three export destinations for each product category.

The largest export category is milk, cream, and concentrated products, which is dominated by China. China is the only country that remains in the top 3 for export value in all four product categories.

\section{$4.2 \quad$ Events}

Here we provide a brief introduction and some background, for each event investigated. We analyse the impact of three product recalls, three adverse weather events and two external events that affected the global dairy market. We are limited in our choice of events, by the time Fonterra's current share trading system has been operating. This is not an exhaustive list of all the potential shocks occurring over this time period; we have selected events based on duration, spread, infrastructure damage, and media coverage. The potential for overlap in estimation or event windows was also considered in the final selection of events.

\subsubsection{Product Recalls}

We investigate three product recalls of varying scope. Ranging from affecting New Zealand sold product only in the Anchor and Pams cream recall, through to the broadly exported product involved in the $80 \%$ whey protein concentrate (WPC80) contamination incident. These were Fonterra's only product recall events to have taken place since the current share trading system came into effect.

\section{Mainland cheese recall}

This recall involved Mainland tasty cheese slices. Mainland is one of Fonterra's major brand names in New Zealand, specialising in cheese and butter. The announcement regarding a packaging defect was made on the 


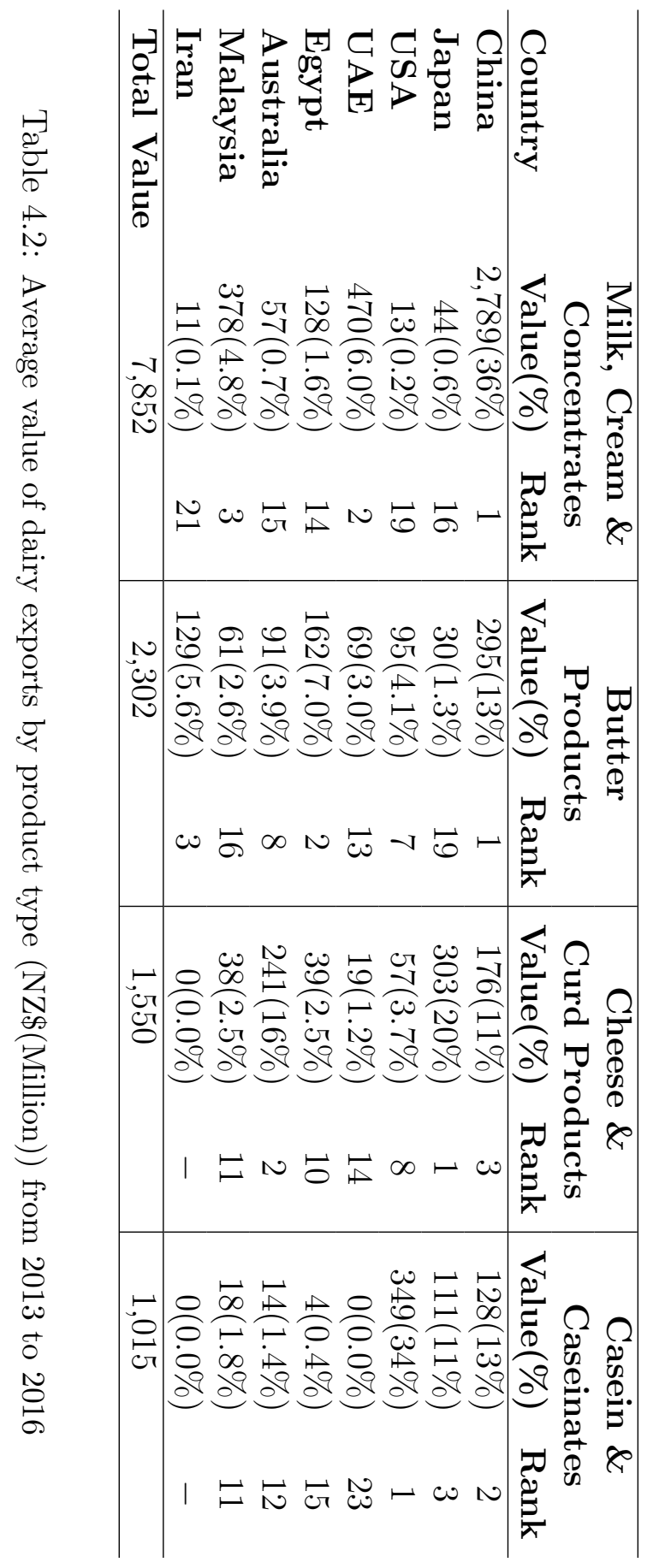


22nd of May 2013 (MPI, 2016). This was the second recall involving Mainland cheese slices in two years (Fox, 2013). The recall was precautionary due to a packaging defect (MPI, 2016). Just one batch of cheese slices was affected. The affected product was mainly restricted to the North Island of New Zealand, though some was exported to Fiji (Fox, 2013). This recall received some media coverage, but this was mostly just repeating the information released in the recall announcement made by the Ministry for Primary industries.

\section{Whey protein contamination incident}

On August 3rd 2013, Fonterra released a statement concerning three batches of (WPC80). They were concerned the product may be contaminated with a botulism causing bacterium, Clostridium botulinum. This was a precautionary recall, but Fonterra was uncertain about the exact location of affected product, and where it may have been used as an ingredient.

In May 2012, a torch was sucked into a processing machine at Fonterra's Hautapu plant. The plant was manufacturing WPC80 at the time. Late in 2012 a customer rejected some product due to high levels of sulphite reducing clostridia. In March 2013 Fonterra launched a technical investigation, following advice that the contaminant may be a botulism causing bacteria. Results of testing were inconclusive. Intermittent discussions were held throughout May and June 2013 regarding the rejected product and other affected product that had been sold. Some limited effort to trace the product were made. On June 26th 2013, AgResearch at Massey University was commissioned to test explicitly for Clostridium botulinum, despite not being accredited for these tests. On July 31st 2013 AgResearch 'confirmed' Clostridium botulinum was present in the affected product. Fonterra initiated a precautionary recall on August 3rd 2013. Finally, twenty five days later on August 28th 2013, Fonterra announced that the affected product had never been contaminated with Clostridium botulinum, but instead with Clostridium sporogenes, which does not cause botulism in humans (Stojkov et al., 2016).

Affected product was manufactured into a bulk ingredient powder and used in infant formula, follow on formula, and grow up milk products, for Nutricia. These products were confirmed as distributed to several countries, including 
Australia, China, and Hong Kong, as well as within New Zealand (WPC 2013 Response Team, 2013).

\section{Anchor and Pams Cream Recall}

Anchor is a major Fonterra brand in New Zealand. Pams is a budget brand who source their dairy products from Fonterra. This recall was announced on the 13th of January 2014 due to the presence of E. coli discovered in product tests. Four batches of cream were affected, packaged in both $300 \mathrm{ml}$ and $500 \mathrm{ml}$ bottles. A total of 8,700 bottles were recalled, though distribution was limited to the upper North Island of New Zealand. There was a relatively large amount of media coverage surrounding this recall, particularly considering the limited volume of product involved. Occurring only a few months after the whey protein incident, there was a lot of discussion regarding potential loss of consumer confidence in Fonterra.

\subsubsection{Adverse Weather Events}

In this section we describe three storms that affected different regions of New Zealand. We considered all of the adverse weather events listed in the New Zealand historic weather events catalogue between 1 March 2013 and 7 April 2017. This is a catalogue of major weather events in New Zealand (NIWA, 2016). Based on conversations with Fonterra personnel, and the nature of an event study, we decided to exclude long term events such as droughts from our analysis. The weather events were then ranked based on the damage they caused in significant dairying regions. The three storms that impacted the most on pasture and farm infrastructure were chosen for this analysis. In each case flooding was a major issue, causing damage to pasture, infrastructure and limiting access. Loss of power is another significant issue, making it difficult to keep milk at an acceptable temperature for collection, leading to loss of product.

\section{September 2013 Storm}

From September 10 to September 12, 2013, an active front crossed New Zealand, bringing strong winds, heavy rain, and thunderstorms. Over forty 
thousand lightning strikes were recorded over the South Island on the 10th of September (NIWA, 2016). Farms in the Canterbury area were hardest hit, with widespread damage to property and powerlines between Darfield and Hororata (Radio New Zealand, 2013). More than eight hundred irrigators were blown over and severely damaged by the strong winds. This damage had serious consequences for farm irrigation and effluent disposal, taking several months to repair. Insurance claims totalled $\$ 74.5$ million, over half of which was for damage to commercial materials, including farm irrigators and silos (NIWA, 2016). Many farms were left without power for days, struggling to milk their cows and to keep the milk cold enough for collection. One farm working with a generator reported having $\$ 30,000$ of milk rejected by a tanker because it was too warm (Anderson, 2013).

\section{July 2014 Northland Storm}

Over four days from 8 July 2014, Northland was subject to severe gales and prolonged heavy rain. Roofs were blown off houses, and over thirteen thousand households were without power, with the rural areas around Kaipara and Whangarei the worst hit. There was heavy rainfall throughout the region; $159 \mathrm{~mm}$ of rain fell in Kaitaia and $117 \mathrm{~mm}$ in Kerikeri. DairyNZ estimated five thousand hectares of grass on dairy farms was under water for up to ten days, taking three months to become productive again (NIWA, 2016).

\section{March 2016 Storm}

Extreme weather affected much of the country on 24 March 2016. The West Coast and Nelson areas in the South Island were affected by flooding. A local state of emergency was declared on the West Coast after nearly two hundred people were evacuated (Radio New Zealand, 2016). Auckland and Northland were subjected to strong winds. The worst hit areas were in Northland, hit by high winds and heavy rain (NIWA, 2016). At one point 24,000 people in the Auckland and Northland regions were without power. Many of these affected regions are strong dairy farming communities, particularly the West Coast and Northland, where many farmers were affected by the power cuts. 


\subsubsection{Global Supply and Demand Shocks}

International events that impact the global supply of milk have the potential to affect Fonterra's share price. particularly as so much of Fonterra's production contributes to the global supply. Fonterra exports $95 \%$ of the dairy goods they produce (MPI, 2014), making up over $18 \%$ of global milk exports (Workman, 2017). We describe in this section two such events. Both events resulted in an increase in global milk supply, but for very different reasons. The circumstances surrounding each of these events occurring was also very different.

\section{Russian Ban on Farm Imports}

On 6 August 2014, Moscow announced a ban on agricultural imports. Starting on 7 August 2014, the Russian Federation introduced import restrictions on a range of agricultural products (European Commission, 2017b), including cheese and milk. Following sanctions placed on Russia for their support of pro-Russian separatists fighting the Ukraine government forces (Gray, 2014), imports from Australia, Canada, the European Union, America and Norway were banned (Boulanger et al., 2015). New Zealand was, and still is, exempt from the ban. The ban on European Union imports has been extended several times and is currently in place until the end of 2017. In January 2017, The Russian Deputy Prime Minister suggested the sanctions might be lifted in 2018. Product produced by the banned countries, that would normally have gone to Russia, suddenly became surplus in the global dairy market. Russia is the second largest global importer of dairy products after China. The equivalent of 2 billion litres of milk was now looking for alternative markets. The negative impacts of the food import ban on Russian domestic markets have forced the Russian government to seek out new trading partners (Khachaturyan et al., 2017).

The next international dairy auction saw prices drop by 6\%. In their August 2014 forecast release, Fonterra maintained their farmgate milk price at $\$ 6$ per $\mathrm{kg}$ MS for 2014/15. This was labelled as optimistic by some commentators. ASB and Westpac put their $\$ 5.80$ forecasts under review, while ANZ dropped their forecast to $\$ 5.25$ per $\mathrm{kg}$ MS (Gray, 2014). In their September 2014 forecast, Fonterra dropped their forecast milk price to $\$ 5.30$ per $\mathrm{kg}$ MS, level 
with Westpac. The forecast farmgate milk price continued to drop after this, it did not increase again at all until May 2015 (Dowson, 2017). The final total payout for the $2014 / 2015$ season was $\$ 4.65$ per $\mathrm{kg}$ MS, the lowest payout since the 2006/2007 season (Interest, 2017).

\section{End of European Union Milk Quotas}

Over the thirty years from 1984, dairy farmers in the European Union operated under the milk quota regime, restricting their production (Hunt, 2015). On 31 March 2015, these quota restrictions were removed (European Commission, 2017a). Farming groups in Ireland were celebrating (Healy, 2015), welcoming the news along with Germany (Hunt, 2015), the Netherlands, and Denmark (Boland, 2015). Not all farmers were so happy however, many were worried expansion of European milk production would flood the market and drop prices (Alltech, 2017). Globally there were similar concerns. The worldwide dairy industry expected the increase in European dairy production to affect dairy farmers in every corner of the globe (International Dairy Foods Association, 2010; Kharpal, 2013). Given that the removal of production caps in the European Union was known about well in advance, this event is likely not a good candidate for an event study. It was not a sudden unexpected event like the other events we consider in this chapter. We have still chosen to conduct this analysis in this case however, to determine if there are any distinguishable reaction in the share price above what was already priced in as shareholders prepared for the cap removal.

\subsection{Methodology and Data}

Event studies have been used extensively for research in accounting, finance, and economics (Gao, 2011). The approach uses financial market data to measure the impact of specific events on the value of a specific firm (MacKinlay, 1997). In this case we are interested in the value of Fonterra. The impact of an event on firm productivity will be immediately reflected in their stock prices. Therefore, we can construct a measure for the economic impact of product recalls using stock prices observed over a relatively short timeframe (MacKinlay, 1997). The events we investigate are described in Section 4.2. 


\subsubsection{Data}

We use several sources of data with an ordinary least squares (OLS) regression to estimate our 'normal' returns model. FCG share prices were obtained from NZX Company Research. Prices are only reported for the days the market was open. Daily returns were calculated as the proportional change in closing share prices between days. For example the return for day $t$ is given by

$$
R_{t}=\frac{\text { Close }_{t}-\text { Close }_{t-1}}{\text { Close }_{t-1}}
$$

Table 4.3 shows the descriptive statistics on daily returns before each shock. Where possible we have used a period of 150 days before each shock; in the case of the Mainland cheese recall and the WPC80 contamination event however, a smaller period of time was necessary due to their occurrence relative to the start of the current share trading system.

\section{Unit Root Tests}

To ensure that our estimation method is meaningful we must first conduct tests for the presence of a unit route in our daily returns series. We use both the augmented Dickey-Fuller (ADF) test (Said \& Dickey, 1984) and the Kwiatowski-Phillips-Schmidt-Shin (KPSS) test (Kwiatkowski et al., 1992). The ADF test is an augmented version of the Dickey-Fuller test for larger, more complex, time series models. The null hypothesis tested assumes there is a unit root present and the data series is not stationary (Said \& Dickey, 1984). The KPSS test is used to assess a null hypothesis that a time series is stationary around a deterministic trend, meaning no unit root is present (Kwiatkowski et al., 1992). The results of these unit root tests are presented in Table 4.4. The $p$-values for each of the ADF tests are very small, and each such test shows a test statistic much more negative than the relevant critical value. This means in each case we can reject the null hypothesis that the series has a unit root. The KPSS tests all report the maximum $p$-value of 0.1 and a test statistic below the critical value of 0.146 . Thus in each case we fail to reject the null hypothesis that the series is stationary.

The results of these tests imply we can consider all the data used in the estimation windows as stationary, with no unit root present. Therefore OLS 


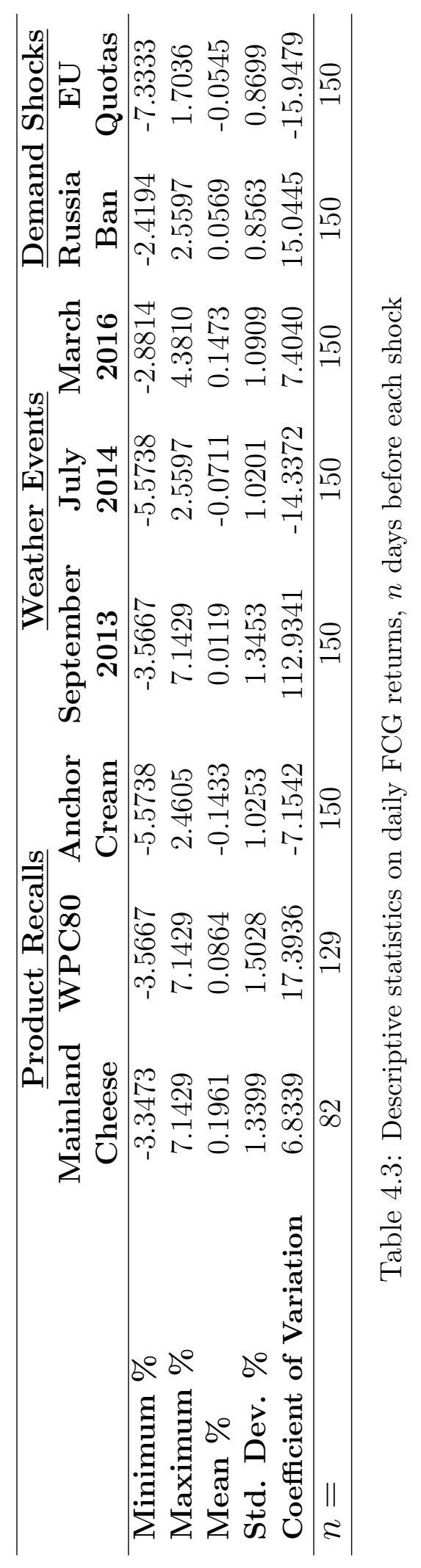




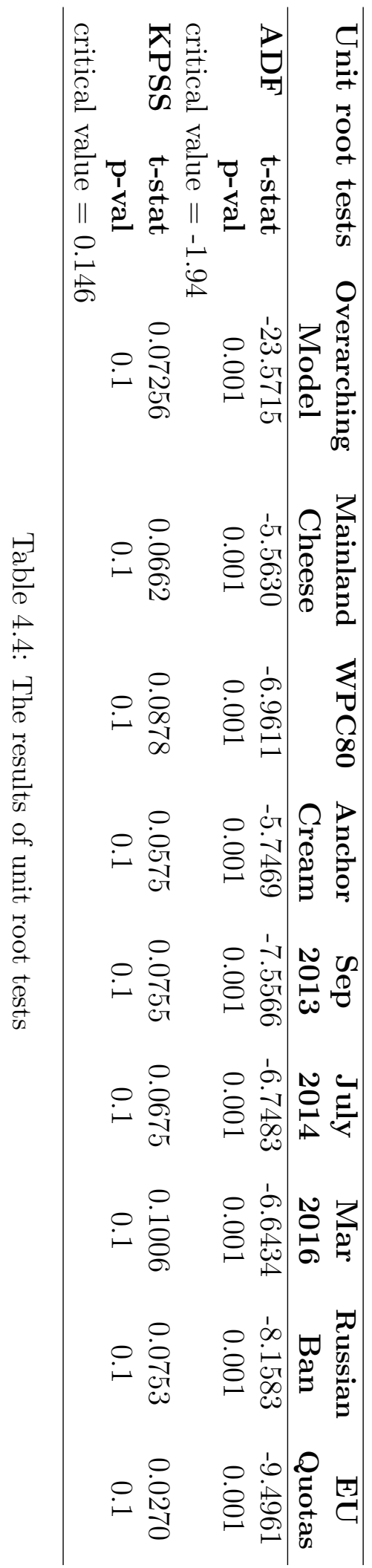


regression is suitable in this case and will not be subject to spurious regression problems.

\section{Regression Data}

The variables used in the OLS regression models throughout the rest of this chapter are described as follows:

$\triangle$ NZX50 The S\&P/NZX 50 index is a measure of the 50 largest eligible ${ }^{1}$ stocks listed on the main board of the New Zealand stock exchange. Fonterra's stocks are not eligible (NZX, 2017b). We use the daily returns for this index in our model estimation. The NZX50 index was obtained from NZX company research (NZX, 2017a).

$\Delta$ Production Because Fonterra's suppliers are required to hold shares proportional to their milk production each season (Fonterra Co-operative Group, 2016b), we consider production as a potential predictor of share price. Monthly data ${ }^{2}$ is obtained from the Dairy Companies Association of New Zealand. We calculate the proportional change from the previous year for a given month to use in the model estimation.

GDT The Global Dairy Trade (GDT) price index provides a reference for the movement of global dairy prices between GDT trading events. While each individual product traded at GDT events has its own index, the GDT Price index ${ }^{3}$ measures the overall price movement for all of them (GDT, 2016b). Auctions, referred to as GDT trading events, operate twice a month (GDT, 2016a).

WMP Whole milk powder (WMP) is a major product traded at GDT events. We consider the WMP price index ${ }^{3}$ as a variable in our model estimation in place of GDT depending on which fits the data better.

\footnotetext{
${ }^{1}$ Only stocks listed on the NZX Main Board are eligible, Fonterra shares are listed in a seperate market. A full explanation of stock eligibility for NZX indices is available here http://us.spindices.com/documents/methodologies/methodology-sp-nzx -index.pdf

${ }^{2}$ The relevant production value for a given month is used for every day in that month, to work in with the daily nature of the other data being used.
} 
AMF Anhydrous milk fat (AMF) is another major product traded at GDT trading events. The AMF price index ${ }^{3}$ is considered in place of the GDT price index or the WMP price index depending on how significantly the model fit is affected.

$\Delta$ Brent Oil Oil price is used as a prediction variable because of its effect on milk demand. When the price of oil drops below a certain level more oil is used for fuel in place of that derived from grain. This grain, originally intended as fuel, is then fed to cows resulting in higher milk production, creating a surplus global milk supply and a drop in global milk prices (Newman \& Mounsey, 2015). Brent oil serves as a major benchmark price for oil worldwide as its value is used to price two thirds of internationally traded crude oil (One Financial Markets, 2017). We use the daily returns on this price as an estimator in our models.

$\triangle$ USD A lot of trade is conducted in American dollars (USD), including GDT trade events. America is also consistently the destination for the second highest value of New Zealand dairy exports. We use the daily returns for the USD exchange rate with the New Zealand dollar (NZD) as a variable in our models.

$\Delta$ CNY China is New Zealand's second largest trading partner for exports. Milk powder has been the largest export commodity since 2008 (Statistics New Zealand, 2016). We therefore use the returns on the Chinese yuan (CNY) exchange rate with the New Zealand dollar as a variable in our models.

$\Delta$ EUR Fonterra does not have a large dairy trade with Europe, however the European Union is another major exporter of dairy and has been projected to surpass NZ as the largest by 2026 (Holloway, 2016). Being such a large exporter we use for the daily returns in the Euro exchange rate with NZD as a variable in our models.

$\Delta$ LKR The returns for the exchange rate between the Sri Lankan Rupee (LKR) with the NZD is considered as they are a long standing major market for Fonterra. Dairy exports to Sri Lanka are worth $\$ 300$ million to New Zealand annually (Draper, 2016). The Sri Lankan rupee is

\footnotetext{
${ }^{3} \mathrm{An}$ explanation of how the GDT price indices are calculated is given in appendix 4.A.
} 
periodically pegged to the USD, so there may be some association. It is however allowed to float freely between pegging and is a significant variable in many regressions where the USD is not. We have therefore chosen to keep the Sri Lankan rupee daily returns as a variable in our regression analyses.

$\triangle$ CLP The proportional change in the Chilean peso (CLP) is considered in our analyses. Fonterra owns $99.4 \%$ of Chile's main dairy brand, Soprole (Fonterra Co-operative Group, 2017b). While Chile is not a consistently high value destination for New Zealand Dairy exports, they did receive the fifth highest value of cheese and curd products exported in the year ending June 2014.

$\triangle$ MYR Fonterra is the leading supplier of dairy ingredients to Malaysian food and beverage manufacturers (Fonterra Co-operative Group, 2017b). Malaysia received the third largest value in New Zealand exports of milk, cream, and concentrated products through the years 2013 to 2016. During the same period Malaysia averaged the fifth highest value of total dairy exports from New Zealand. We therefore consider the proportional change in the Malaysian ringgit (MYR) in our models.

$\triangle$ JPY Japan was the destination for the third greatest value of total New Zealand dairy product exports over the years 2013 to 2016. Japan consistently receives the largest value of cheese and curd products. Because of this we consider daily returns for the the Japanese yen (JPY) in our regression analyses.

$\triangle$ AUD Australia is Fonterra's largest milk pool outside of New Zealand and also the recipient of the second largest value of New Zealand cheese and curd exports. The proportional daily change in the exchange rate with the Australian dollar (AUD) is thus considered as a variable in our regression analyses.

$\triangle \mathbf{P H P}$ The Phillipines received the fourth greatest value of total New Zealand dairy exports in the years ending June 2013 and 2014. Because of this we consider the daily returns on the exchange rate with the Philippine peso (PHP) as a variable in our models. 
Month Dairy is a very seasonal industry, with huge variation in production throughout the year. Therefore we use a dummy variable for each month of the year. We also allow for interaction terms between month and several relevant variable such as production, oil price, and currency exchange rates.

Several other predictor variables were considered but not found significant. These include the forecast farmgate milk price, the skim milk price index, and the West Texas intermediate oil price. Several other currency exchange rates for markets important for Fonterra were also considered.

\subsubsection{Model Construction}

We use two types of model for comparison in our event analysis, an 'overarching' model estimated over the whole length of the data, and a specific 'individual event' model, estimated over fifty to seventy five days preceding the relevant event.

It is critical to isolate the day on which the stock market became aware of the shock, in order to correctly estimate any effects. An efficient market will react to news quickly, so a window that begins too late will miss any immediate effects. Depending on the type of shock, it is also possible that information will leak through to the stock market before any official announcement. We can allow for this by starting the event window an appropriate number of days before the shock announcement (MacKinlay, 1997). This is particularly applicable for storms, which are normally forecast. Among the recalls we investigate, none appear to have been leaked, though the WPC80 contamination incident involved several different parties outside of Fonterra in detecting the problem. ${ }^{4}$ This increased the potential for rumours to get out before the official announcement (WPC 2013 Response Team, 2013). The removal of production caps in the European Union was known about well ahead of time, but there was potentially doubt around whether it would actually happen. We do not know of any leak regarding the Russian ban on

\footnotetext{
${ }^{4}$ An analysis of the WPC80 contamination incident by Stojkov et al. (2016) suggests a decline in exports that started sometime before the contamination and recall were announced.
} 
farm imports, however it was largely a response to sanctions, to which some retaliation was likely expected.

In the case of the overarching model, a twenty five day period surround each event is excluded from the model estimation, five days before to twenty days following, $(t-5, t+20)$, to allow for valid comparison, where $t$ is the the day of the event. The estimation period for the seventy five day model would be $(t-81, t-6)$, accounting for a five day pre-event window. The model is then extended over the event window, in this case $(t-5, t+20)$, and the estimated returns are compared with the actual returns. In the case where other events may occur within an estimation window, the entire event window associated with the 'interrupting' event is excluded from the model estimation. We use an OLS regression to estimate these models. We mentioned in Section 4.1.1, the use of a GARCH model by Gao (2011). For most of our models however, White's test does not detect the presence of heteroskedasticity, thus OLS regression was deemed sufficient in this case (White, 1980). The general model specification for the estimated normal returns $\widehat{R}_{t}$ is

$$
\widehat{R}_{t}=\alpha+\sum_{i}^{j} \beta_{i} x_{i t}+\varepsilon_{t}
$$

where $\alpha$ is the intercept value, $x_{i t}$ is the value of a given variable $i$ on day $t$ with $\beta_{i}$ describing its relationship to the daily returns, and $\varepsilon_{t}$ is the residual value for the model at time $t$.

Each model is built using a bottom up approach. Each variable was regressed against the share price return data in the appropriate estimation window, the variable that best fit the share price data was then included as the first variable in the model. The square of each variable is also considered. Subsequent variables were then added to the model in order of how well they fit in the individual regressions and either confirmed or rejected based on how much they improved the existing model. The model is deemed complete when no other variable improves the model to an extent that is worth the loss of degrees of freedom. To avoid issues with significant correlation between variables, specific variable are excluded from being used in the same model, this applies particularly the GDT price and its components. 


\subsubsection{Event Study Methodology}

Event-study methodology is outlined in Campbell et al. (1997). For each event we estimate the cumulative abnormal returns relative to each of the two models. We estimate the abnormal rate of returns $\left(\widehat{A R_{i t}}\right)$ as the difference between the 'normal' rate of returns, as predicted by the model, and the actual rate of returns, for time $t$, in the event window (Gao, 2011), as in Equation 4.1, where $i$ denotes the specific event effect we are analysing and $t$ is the day.

$$
\begin{gathered}
\widehat{A R}_{i t}=R_{i t}-\widehat{R}_{i t} \\
C \widehat{A R}_{i}=\sum_{t_{1}}^{t_{2}} \widehat{A R}_{i t}
\end{gathered}
$$

The cumulative abnormal returns is given by $C \widehat{A R}_{i}$ in Equation 4.2 . It is the sum of the abnormal returns from day $t_{1}$ to day $t_{2}$. The opening of the event window is denoted by $t_{1}$, and the closing by $t_{2}$. The statistical precision of these abnormal returns is strongly dependent on the fit of the normal returns model, and the length of the post-event window.

The variance of the cumulative abnormal return is given by

$$
\begin{aligned}
\operatorname{Var}\left[C A R_{i}\right] & =\operatorname{Var}\left(\sum_{t_{1}}^{t_{2}} A R_{i t}\right) \\
& =\sum_{t_{1}}^{t_{2}} \operatorname{Var}\left(A R_{i t}\right) \\
& =\left(t_{2}-t_{1}+1\right) \sigma_{\varepsilon_{i}}^{2}
\end{aligned}
$$

where $\sigma_{\varepsilon_{i}}^{2}$ is the variance of the residuals $\varepsilon_{i t}$ from the normal returns regression model (Salinger, 1992). We use the value for the cumulative normal returns, as obtained from Equation 4.2, to test the null hypothesis that the abnormal returns are not statistically distinguishable from zero (MacKinlay, 1997), as stated in Equation 4.3.

$$
H_{0}: C A R_{i}=0 \quad H_{1}: C A R_{i} \neq 0
$$


We estimate the cumulative abnormal returns over several time spans preceding and following each event and test them for statistical significance. Pre-event windows of five days, three days, and one day are analysed along with post-event windows of three days, five days, ten days fifteen days, twenty days, and twenty five days. The significance of these cumulative abnormal returns is calculated using the test statistic in Equation 4.4. This significance is dependent on the fit of the model in the estimation window. The formula used for the test statistic $\theta_{i}$, is

$$
\theta_{i}=\frac{C \widehat{A R}_{i}}{\sqrt{\operatorname{Var}\left(C \widehat{A R}_{i}\right)}}=\frac{C \widehat{A R}_{i}}{\sqrt{\left(t_{2}-t_{1}+1\right) \sigma_{\varepsilon_{i}}^{2}}}
$$

Under the null hypothesis, $\theta_{i}$ has the Student's t distribution with $T-2$ degrees of freedom, where $T$ is the length of the pre-event estimation window (MacKinlay, 1997).

\subsection{Analysis and Results}

In this section we analyse the impact of each shock on FCG share price returns. For each shock we compare the actual returns to normal returns as predicted by an 'overarching' model and an 'individual event' model in order to estimate the abnormal returns. Each model is estimated using an OLS regression.

\subsubsection{Overarching model}

We first fit a model to the whole daily returns on FCG share price data set, between 1 March 2013 and 7 April 2017. While Fonterra's current stock trading system came into effect in December 2012, there was rather intermittent trading initially. Of the 62 trading days up to 1 March 2013, only 26 days saw any movement of stock. Therefore we choose to start estimating our models once trading appeared more settled. We exclude the days surrounding each event in this regression, specifically the five days before each event and the twenty days following. Using the predictor variables described in Section 4.3 
we specify the overarching model below

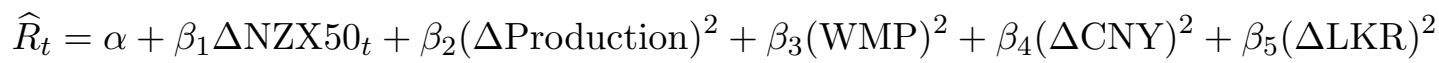

$$
\begin{aligned}
& +\sum_{i=2}^{12}\left(\gamma_{1 i} \text { Production }_{i t}+\gamma_{2 i} \Delta \text { Brent Oil }_{i t}+\gamma_{3 i} \Delta \mathrm{USD}_{i t}+\gamma_{4 i} \Delta \mathrm{EUR}_{i t}\right)+\varepsilon_{t}
\end{aligned}
$$

where $i=2, \ldots, 12$ corresponds to the months February through December. We allow some interactions with the month of the year as dairy production and exports are very seasonal. It is logical that production and exchange rates will matter more at the times of the year when the most product is being produced and exported to relevant regions. The parameter values and standard errors resulting from the OLS regression on are given in Table 4.5.

\begin{tabular}{lcc}
\hline Variable & $\begin{array}{c}\text { Parameter } \\
\beta_{i}\end{array}$ & $\begin{array}{c}\text { Standard } \\
\text { Error }\end{array}$ \\
\hline$\Delta$ NZX50 & $-0.10517^{*}$ & 0.06528 \\
$(\Delta \text { Production })^{2}$ & $0.04313^{* *}$ & 0.02331 \\
$(\mathbf{W M P})^{2}$ & $-0.00142^{* *}$ & 0.00070 \\
$(\Delta \mathbf{C N Y})^{2}$ & $12.14775^{*}$ & 6.37328 \\
$(\Delta \mathbf{L K R})^{2}$ & $-19.39685^{* * *}$ & 7.28947 \\
$\Delta$ Production $\times$ Month & - & - \\
$\Delta$ Brent Oil $\times$ Month & - & - \\
$\Delta$ USD $\times$ Month & - & - \\
$\Delta \mathbf{E U R} \times$ Month & - & - \\
Intercept & $0.00193^{* *}$ & 0.00078 \\
\hline$R^{2}$ & 0.095 & \\
\hline$n=801$ & & \\
${ }^{*}$ statistically significant at the $10 \%$ level & \\
$* *$ statistically significant at the $5 \%$ level & \\
$* * *$ &
\end{tabular}

Table 4.5: Parameters of the normal returns model for the overarching model. In the case of the month interaction terms, see Appendix 4.B for the full model.

White's test indicates the presence of heteroskedasticity, therefore we use heteroskedasticity and autocorrelation consistent (HAC) standard errors in this model (White, 1980).

We will use this model, along with models derived for the time period immediately preceding each event, to estimate the impact these shocks have on 
FCG share returns. The explanatory power of this model is relatively low $\left(R^{2}<0.1\right)$ meaning less than $10 \%$ of the variation in the data is explained by this model. The test statistic we use however (Equation 4.4), takes into account the fit in the estimation window. This reduces the likelihood of type I errors where we might see significant abnormal returns that are not actually significant. The potential for type II errors is increased however, meaning if we see significant abnormal returns it is likely that they actually are significant, however some significant abnormal returns that would be detected with a better fitting model may be missed.

\subsubsection{Product Contamination and Recalls}

In this section we analyse the cumulative abnormal returns surrounding each of the recall incidents we have chosen to investigate. We estimate individual event models for the 2013 Mainland cheese recall, the WPC80 contamination incident in 2013, and the Pams/Anchor Cream Recall in 2014. These models, along with the overarching model from Section 4.4.1, are then used to estimate the abnormal returns surrounding each event and their significance.

\section{Individual Recall Event Models}

The mode specification for the individual recall events is

$$
\begin{aligned}
\widehat{R}_{t}= & \alpha+\beta_{1} \Delta \mathrm{USD}_{t}+\beta_{2} \Delta \mathrm{JPY}_{t}+\beta_{3} \mathrm{GDT}_{t} \\
& +\beta_{4}(\Delta \text { Brent Oil })^{2}+\beta_{5}(\mathrm{EUR})^{2}+\varepsilon_{t}
\end{aligned}
$$

The parameter values are estimated for each individual recall event model separately. Table 4.6 shows the parameter values and standard errors for each individual recall event. A seventy five day estimation window is used where possible, though in the case of the Mainland cheese recall, only fifty days of data is suitable for use in the estimating the model. Because of the seasonal nature of dairy, we cannot use too long an estimation period without accounting for the time of year. Unless the estimation period is longer than a year however including seasonal parameters means we will likely be applying the seasonal model to a time we have not estimated seasonal parameters for. 
This length of estimation window also appears to be one of several standard estimation windows for food related events and their effect on share prices. In particular, seventy five days is the estimation period used by Salin \& Hooker (2001).

The Mainland cheese recall was relatively small, though there was some international distribution of product. The parameters for this model were estimated based on the fifty days preceding the five day pre-event window before the recall announcement. This takes us back the earliest of the FCG returns data we are able to use. The square of the Brent oil price is the only variable statistically significant at the $1 \%$ level for this section of the data. Oil prices can have a large influence on global surplus milk, so it is reasonable that the proportional change in oil price could affect Fonterra's share price returns. The proportional change in the Japanese Yen is significant at the $5 \%$ level in this case; interestingly Japan consistently receives the the largest value of New Zealand cheese and curd products. While the Chinese yuan is not significant in the case, the proportional change in the American dollar is significant at the $10 \%$ level. White's test does not indicate the presence of heteroskedasticity in this case, therefore we do not use HAC standard errors (White, 1980).

The WPC80 contamination incident was a much larger event than the Mainland cheese recall in terms of publicity, spread, and the value recalled. The product was widely distributed internationally and involved bacteria with the potential to cause serious illness. This was also an ingredient in many other products. In order to estimate the model for the FCG returns around this event we use an estimation window finishing five days before the contamination announcement, we omit the post-event window from the May 2013 cheese recall, and continue back for a total of seventy five days. The square of the proportional change in Brent oil price is still significant at the $1 \%$ level in estimating the returns around the WPC80 contamination incident. The proportional change in the Japanese yen and the American dollar are now both significant at the $10 \%$ level. Again White's test does not detect the presence of heteroskedasticity, so we do not use HAC standard errors (White, 1980).

The model for the returns around the Pams and Anchor cream recall involves the relative change in the Euro exchange rate and the GDT price index. The 


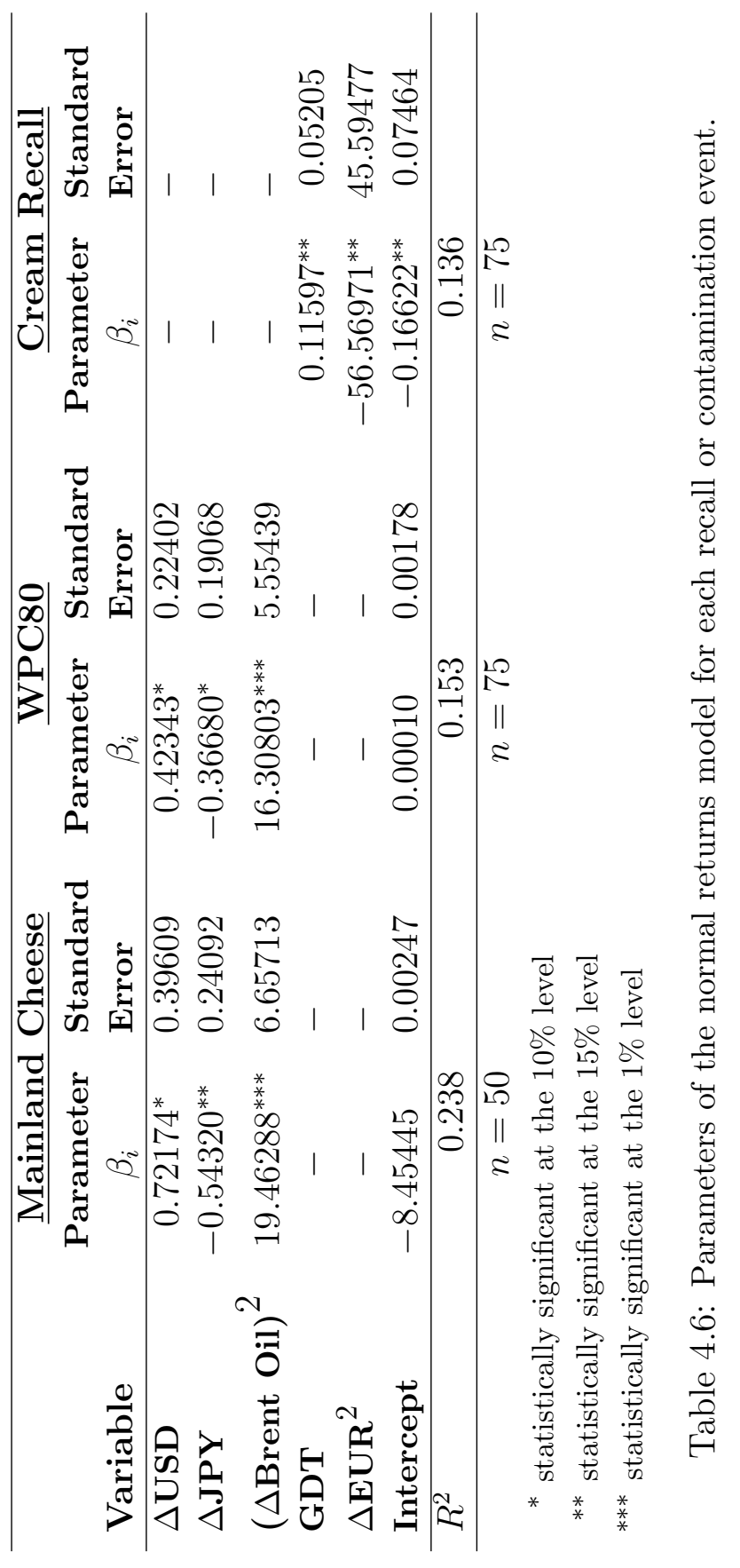


GDT price index contains all of the AMF, butter, SMP and WMP price indices, and in this regression is more significant as a whole than as some combination of its parts. We use a seventy five day estimation window that ends five days before the recall announcement. The estimation window in this case has no overlap with either of the earlier events, therefore we do not need to omit any days.

\section{Recall Event Plots}

We plot the actual returns along with the normal returns, as predicted by the 'overarching' model and the relevant 'individual event' model for each recall or contamination event.

Figure 4.1 shows the normal returns around the Mainland cheese recall, as estimated by the model in Table 4.6 and the overarching model from Table 4.5, along with the actual FCG returns.

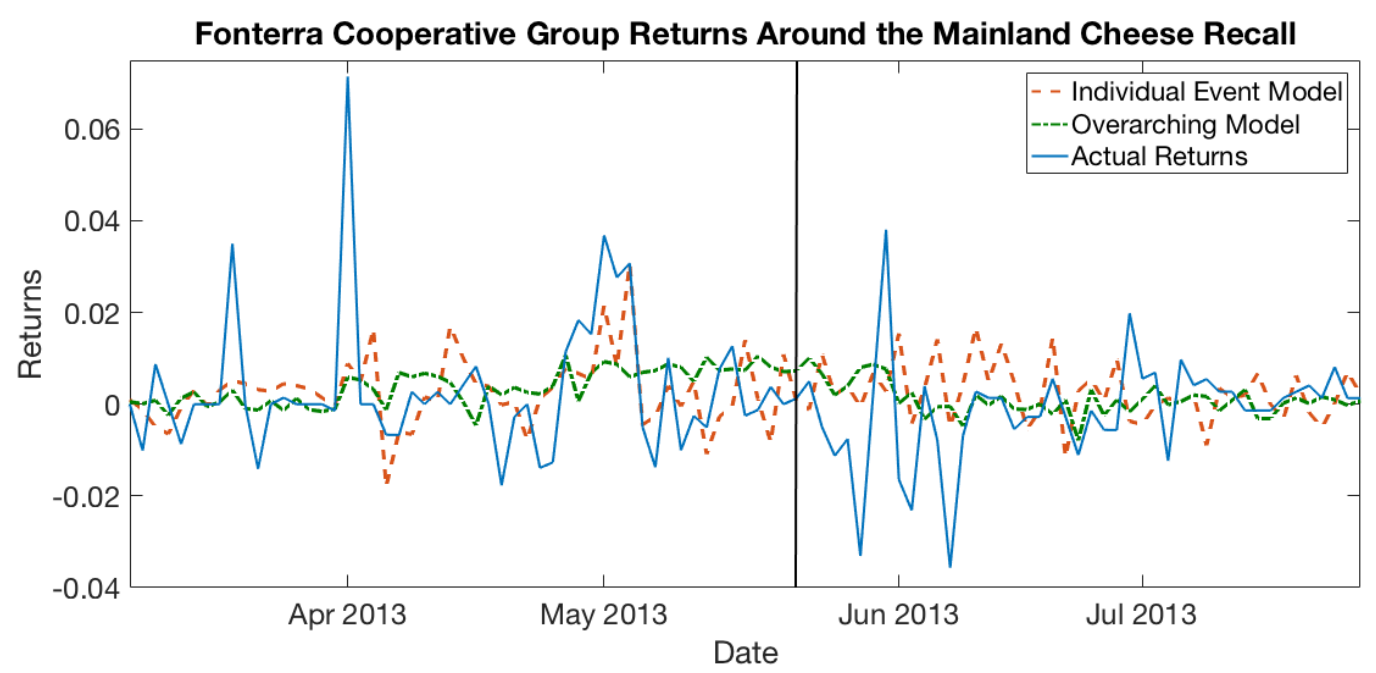

Figure 4.1: Actual FCG returns plotted along with the normal returns as estimated by the fifty day individual event model and the overarching model. The vertical black line marks 22 May 2013, the day the Mainland cheese recall was announced.

A visual analysis of this plot suggests there may be significant drop in returns in the days following the recall announcement. The returns predicted by both models hold fairly steady above the actual returns for a time following the Mainland cheese recall announcement. In the case of the overarching model, these higher predicted returns appear to start a few days before the recall 
announcement. This is not the case of the individual event model, which does not show any significant deviation from the predicted normal until after the recall announcement. This model does sit higher then the actual returns for some time following the recall announcement. The actual returns show a drop immediately after the recall that is not reflected in either model.

Figure 4.2 shows the normal returns predicted by each model, for the days around the WPC80 contamination announcement plotted alongside the actual returns.

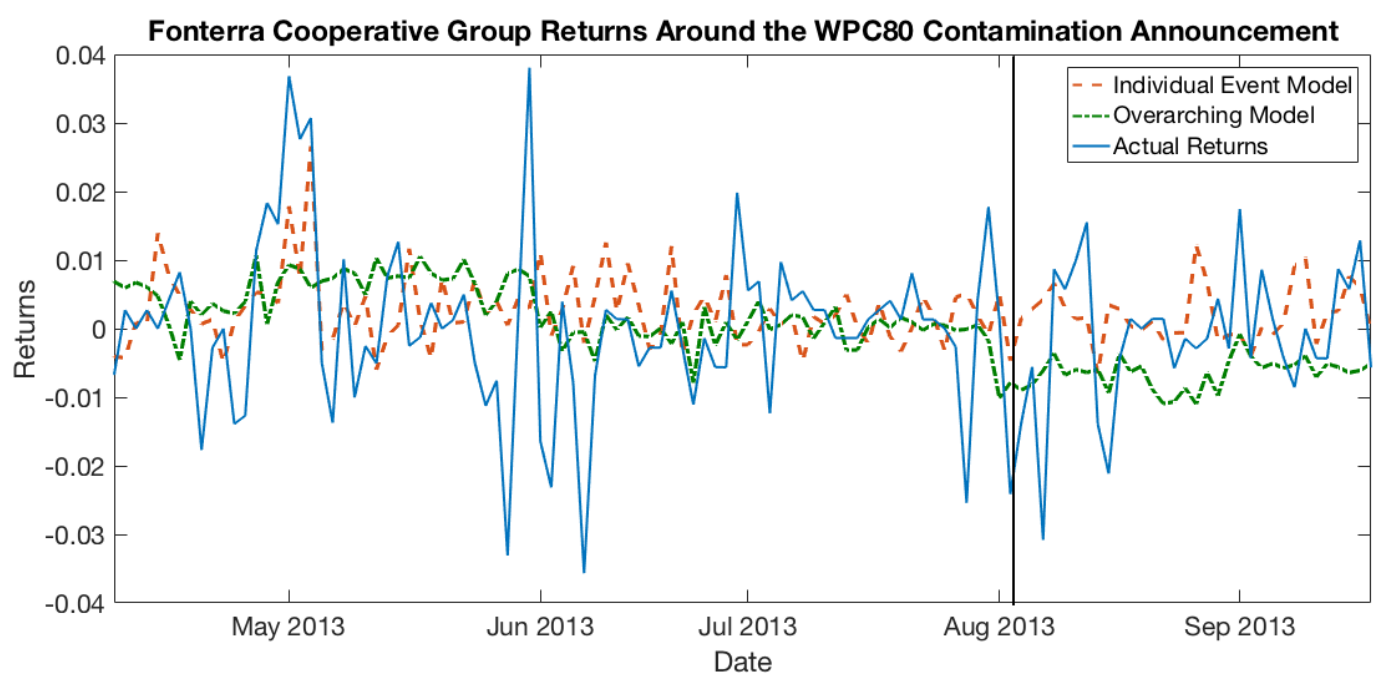

Figure 4.2: Actual FCG returns plotted along with the predicted normal returns using the seventy five day individual event model and the overarching model. The vertical black line marks 3 August 2013, the day the WPC80 contamination incident was announced.

Looking at this this plot, there appears to be a significant drop in returns in the first few days following the contamination announcement, particularly compared to the individual event model, but things return to normal fairly quickly. It also appears that the drop may have begun before the announcement. This drop is also partially mirrored by the overarching model. There is a series of small positive spikes in actual returns about fifteen to twenty days after the contamination announcement, though cumulatively the abnormal returns are not statistically significant. This incidentally starts on August 28 2013, the date the contamination was declared a false alarm. The overarching model seems to underestimate returns for quite some time following the announcement.

Figure 4.3 shows the actual returns around the Pams/Anchor cream recall 
in January 2014, plotted alongside the normal returns predicted using the relevant model presented in Table 4.6.

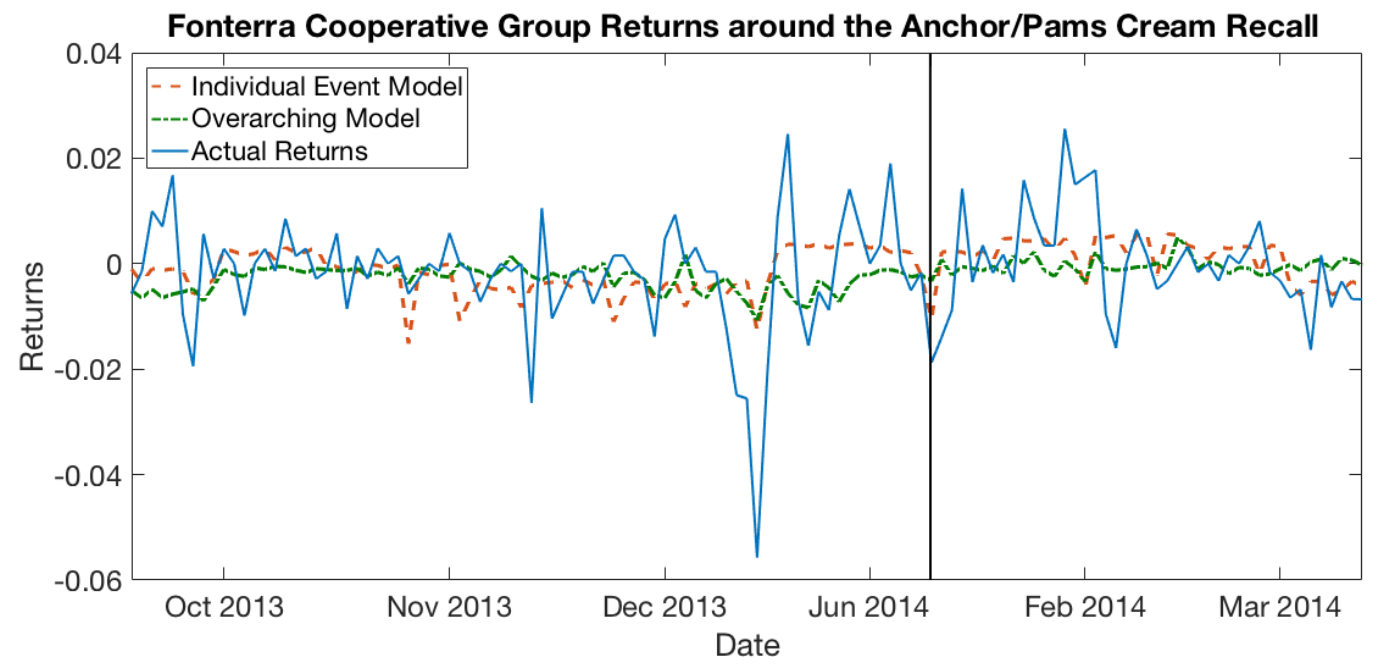

Figure 4.3: Actual FCG returns plotted along with the normal returns as predicted by the seventy five day individual event model from Table 4.6. The vertical black line marks 13 January 2014, the day the cream recall was announced.

Visually there do not appear to be any significant differences between the actual returns and the predicted normal returns, either before or after the day the cream recall was announced regardless of whether we use the overarching model or the individual event model.

\section{Recall Event Cumulative Abnormal Returns}

We analyse the significance of the cumulative returns around each event in Table 4.7. Analysis is shown for five, three, and one day pre-event windows, before the event occurred in order to asses any anticipatory response. The event day itself is also shown along with one, three, five, ten, fifteen, and twenty day post-event windows. The null hypothesis in each case in each case being that cumulative abnormal returns are equivalent to zero, as stated in Equation 4.3. 'Over' refers to the overarching model, we have shortened this term for use in this and later tables.

We do not see any significant cumulative abnormal returns in the lead up to the Mainland cheese recall compared to either model. Following the recall we start to see abnormal cumulative returns significantly different from zero after five days. This coincides with the bottom of the first drop in returns 


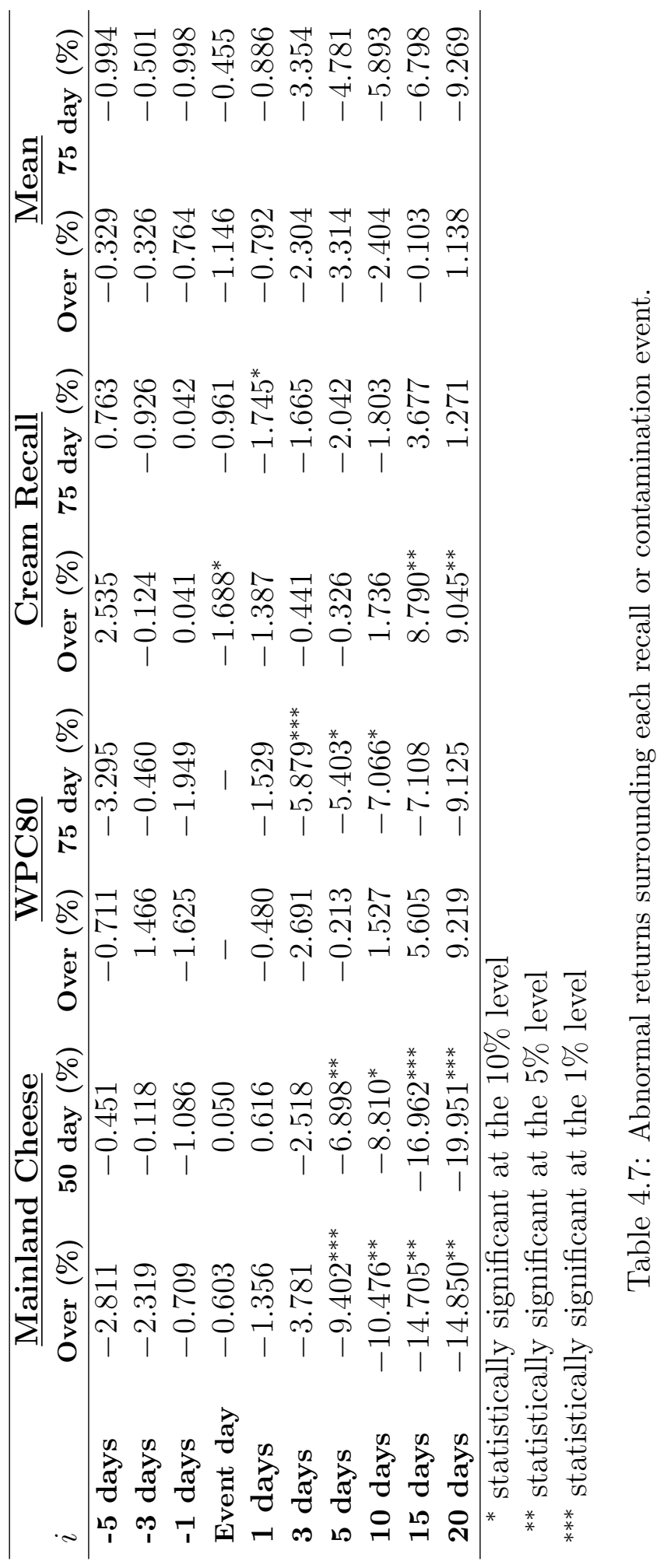


seen in Figure 4.1. These cumulative abnormal returns are significant and negative for both models, however only at the $5 \%$ level for the fifty day individual event model versus $1 \%$ for the overarching model. The drop is counteracted briefly by the peak seven days after the recall announcement, but the subsequent drops in returns on post-event days nine and twelve, mean we see significant cumulative abnormal returns over the rest of the twenty day post-event window. From day ten to day twenty both models show significant cumulative abnormal returns.

As it appeared in Figure 4.2, comparison with the overarching model shows no significant cumulative abnormal returns in the days surrounding the WPC80 contamination announcement. Using the seventy five day individual event model from Table 4.6 we do not see any significantly abnormal returns before the contamination was announced. We do not see any significant abnormal returns the day after the announcement, however the three day post-event window shows negative cumulative abnormal returns significant at the $1 \%$ level. This along with what we observe in Figure 4.2 suggests most of the abnormality is due to the negative spike in returns on August 72013 , three business days after the contamination announcement. We did not see this significance in comparison with 'overarching' model in Table 4.7, we did see an increase in cumulative abnormal returns for the three day post-event window, however it was not enough to be significant. We do see significant cumulative abnormal returns at the $10 \%$ level on post-event days five and ten, but nothing after this. Such a small share price reaction, to an event that involved several countries and high publicity, is rather puzzling. It is beyond the scope of this thesis, however an investigation into why we see such a small response could prove interesting.

There is no significant departure from the predicted normal returns in the five days prior to the Pams/Anchor cream recall announcement for either normal returns model, meaning we are not seeing the effects of any information leakage. The overarching model shows a negative abnormal return, significant at the $5 \%$ level on the day of the recall announcement, while a similar reaction is seen the day after the announcement in comparison with the individual event model. Cumulatively both models show significant returns over these two days, the overarching model at the $5 \%$ level and the individual event model at the $10 \%$ level. The overarching model shows sig- 
nificant positive cumulative abnormal returns for the fifteen and twenty day post-event windows. While the individual event model also shows positive cumulative abnormal returns at this time, they are not significant at all.

If we average the cumulative abnormal returns across the recall events, we do not see significance for any of the pre-event or post-event windows, for either of the models. This suggests there is no consistent reaction to this type of event.

\subsubsection{Adverse Weather Events}

In this section we assess the financial impact of three adverse weather events. Each event is a storm that resulted in sustained flooding and/or significant damage to farm infrastructure, affecting areas of New Zealand important to dairy production.

\section{Individual Adverse Weather Event Models}

The mode specification for the individual adverse weather events is

$$
\begin{aligned}
\widehat{R}_{t}= & \alpha+\beta_{1} \Delta \mathrm{USD}_{t}+\beta_{2} \Delta \mathrm{MYR}_{t}+\beta_{3} \Delta \mathrm{LKR}_{t}+\beta_{4} \mathrm{AMF}_{t} \\
& +\beta_{5}(\Delta \mathrm{EUR})^{2}+\beta_{6}(\Delta \mathrm{LKR})^{2}+\beta_{7}(\Delta \mathrm{CLP})^{2}+\varepsilon_{t}
\end{aligned}
$$

The parameters, as estimated for each individual event using OLS regression, are given in Table 4.8. A seventy five day estimation window is used in each case.

Occurring over three days from 10 to 12 September 2013, this storm caused significant damage to property and power lines. Canterbury farms were the hardest hit. Being a weather event we also allow for some forecast regarding the event as well. A five day pre-event window is used in this case. The first column in Table 4.8 shows the linear regression model leading up to this weather event.

The American dollar, the Malaysian ringgit, and the Sri Lankan rupee are all significant at the $5 \%$ level for this section of the FCG returns data. The United States is consistently the second top destination for New Zealand dairy exports and a lot of international sales are conducted in American 


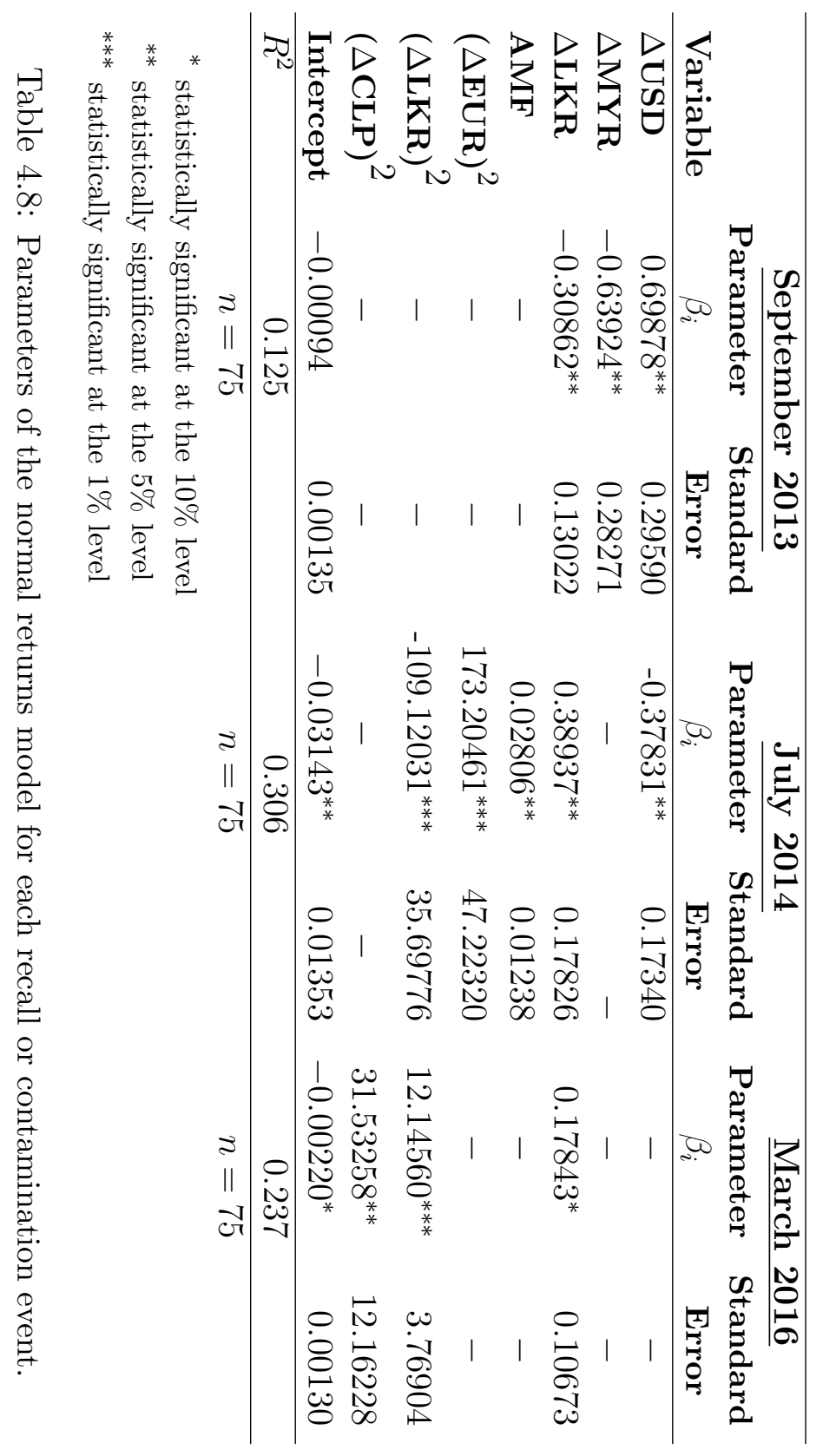


dollars. Over the 2013 to 2016 trading seasons Malaysia averages fifth in terms of New Zealand dairy export value. Sri Lanka is a significant, though not top five, destination for New Zealand dairy products. This market was heavily affected by product bans in August 2013 after Sri Lanka's ministry of health said two batches of milk powder had tested positive for dicyandiamide, a nitrification inhibitor used in fertilisers (Reuters, 2013). This may partially explain the negative parameter value in this model. This also increases the need for caution in interpreting the abnormal returns estimated using this model. White's test does not detect any heteroskedasticity for this model, therefore we do not need to use HAC standard errors (White, 1980).

The July 2014 storm arrived on 8 July and lasted until 11 July 2014. Significant damage including loss of power and flooded pasture resulted. The model fitted to the estimation window for this event is given in Table 4.8. The seventy five day estimation window leading up to the arrival of this storm gives a model with several significant parameters. The most significant parameters are the daily returns on the Euro and the Sri Lankan rupee, squared. The AMF price index, another component of the GDT price index, is also significant in this model. White's test does not detect any heteroskedasticity for this model, therefore we do not need to use HAC standard errors (White, 1980).

The third storm we analyse arrived in New Zealand on 24 March 2016. While this storm only lasted for one day, much of the country was affected. Northland however was the most affected region. The seventy five day model used for this event is given in the last column of Table 4.8. In this case the square of the Sri Lankan rupee is the most significant parameter, significant at the $1 \%$ level. The Chilean peso is a significant estimator at the $95 \%$ level. The year ending June 2016 was particularly good for exports to Sri Lanka, comprising fifth greatest value of milk, cream, and concentrated products exported. The proportional change in the Sri Lankan rupee is the only other significant parameter. Again the presence of heteroskedasticity is not detected.

\section{Adverse Weather Event Daily Return Plots}

The 'normal' returns, as predicted by the 'overarching model' and the relevant individual event model, are plotted along with the actual returns for 
each adverse weather event. The returns around the September 2013 storm, as predicted by the model in Table 4.8, are plotted in Figure 4.4 along with the actual returns for this time period. Looking at this plot, there do not

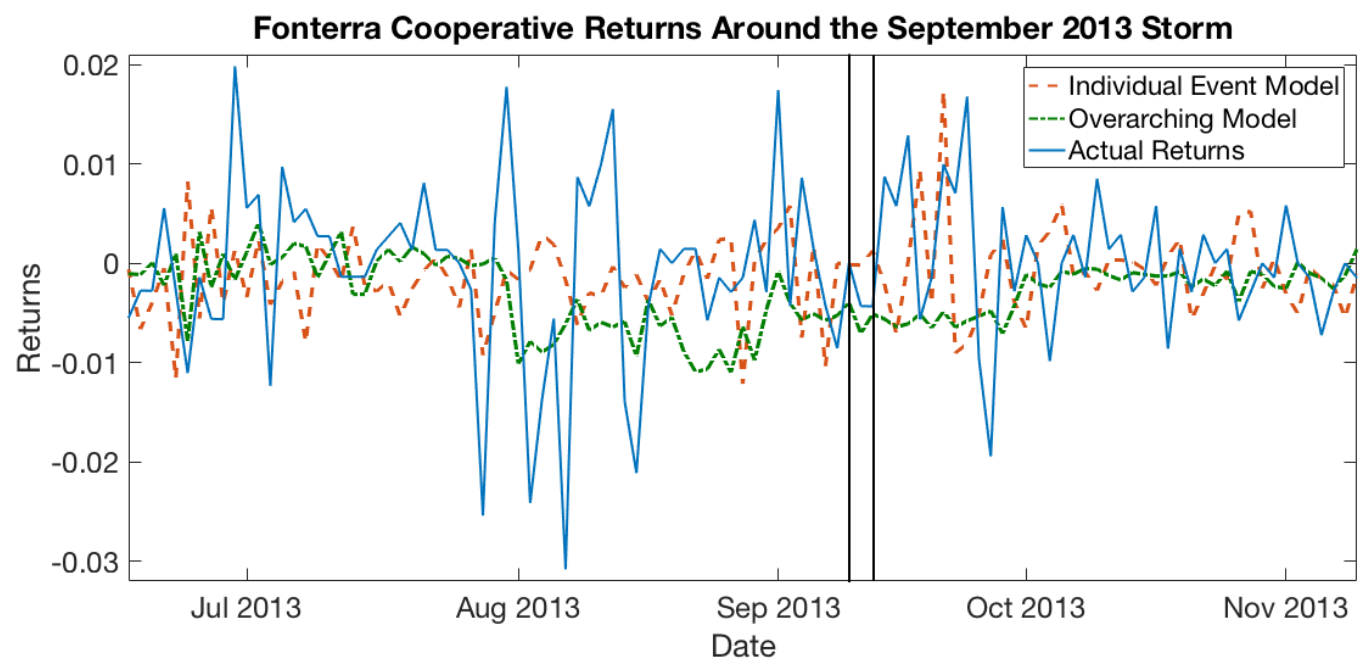

Figure 4.4: Actual FCG returns plotted along with the returns predicted by seventy five day event model. The first vertical black line marks 10 September 2013, the day the storm hit. The second line marks 12 September 2013, the end of the storm.

seem to be any negative effects on FCG returns surrounding the September 2013 storm, in comparison with either the overarching or the specific individual event model. There actually appears to be a positive effect due to the storm in the first twelve days after it ended. These positive abnormal returns are even more apparent in comparison with the overarching model. After this it does not look like there was any significant impact, either positive or negative, due to this storm.

Figure 4.5 shows the returns predicted using the model presented in Table 4.8 for the July 2014 storm, plotted against the actual returns. There do not appear to be any significant abnormal returns in the five days before the storm or during the storm in comparison to the individual event model, though there does appear to be a slight negative effect compared to the overarching model. There do appear to be sustained positive abnormal returns in the days following the storm compared to both models. This is more pronounced in comparison with the individual event model.

Figure 4.6 shows the normal returns for the March 2016 storm, as predicted by the individual event model presented in Table 4.8 and the overarching 


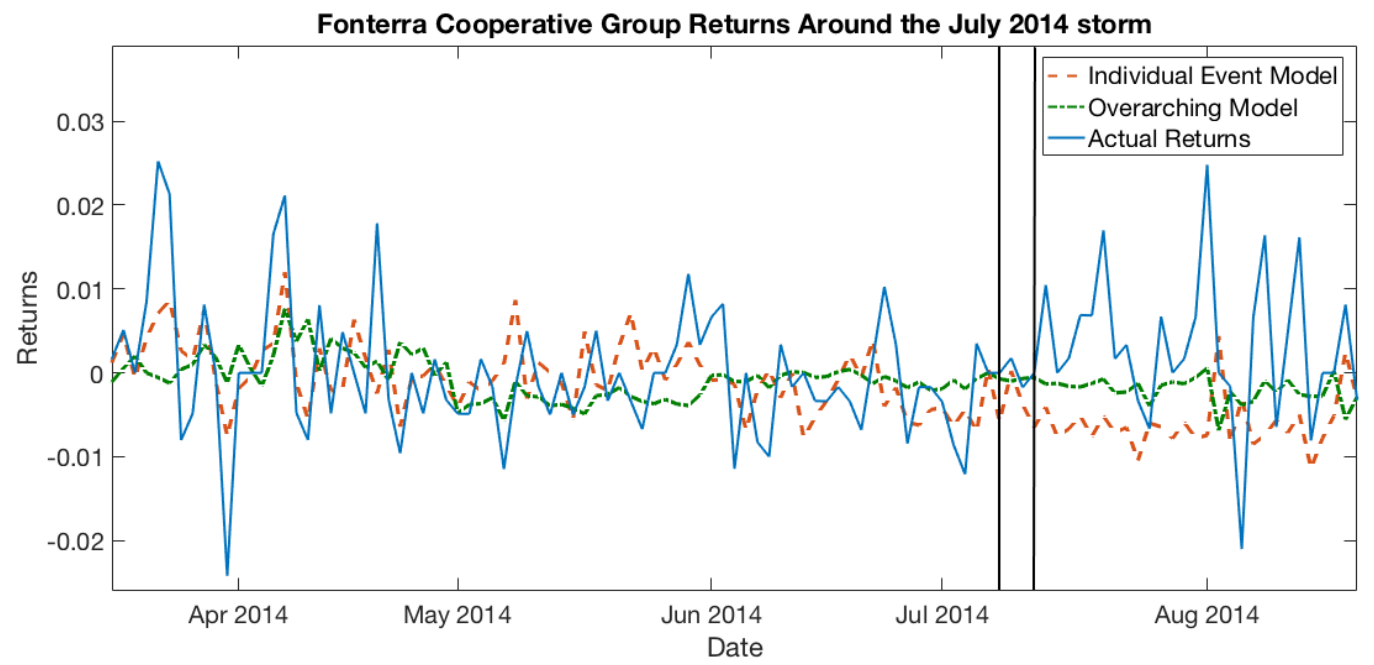

Figure 4.5: Actual FCG returns plotted along with the seventy five day normal returns model. The first vertical black line marks 8 July 2014, the first day of the storm. The second line marks 11 July 2014, the end of the storm

model from Table 4.5, plotted alongside the actual returns for this time period. Apart from a couple of spikes seen in the model prediction, the actual returns do not seem to show any significant departure from from what either model predicts would be normal in either the pre-event or the postevent period.

\section{Adverse Weather Event Cumulative Abnormal Returns}

The cumulative abnormal returns from adverse weather events, for various pre- and post-event windows, are given in Table 4.9. As stated in Equation 4.3 , statistically significant cumulative abnormal returns means we can reject the null hypotheses that they are zero. Again the 'Over' refers to the 'overarching' model.

Looking at the 'overarching' normal returns model around the September 2013 storm in the first column of Table 4.9, we see cumulative abnormal returns significant at the $10 \%$ level for all post-event windows from three to twenty days. For the eight and ten day windows these abnormal returns are significant at the $5 \%$ level. This matches with the end of the two positive spikes we see in the actual returns in Figure 4.4. Neither of the two spikes are significant on their own however, but in aggregate they become so. In comparison the individual event model only shows significant cumulative ab- 


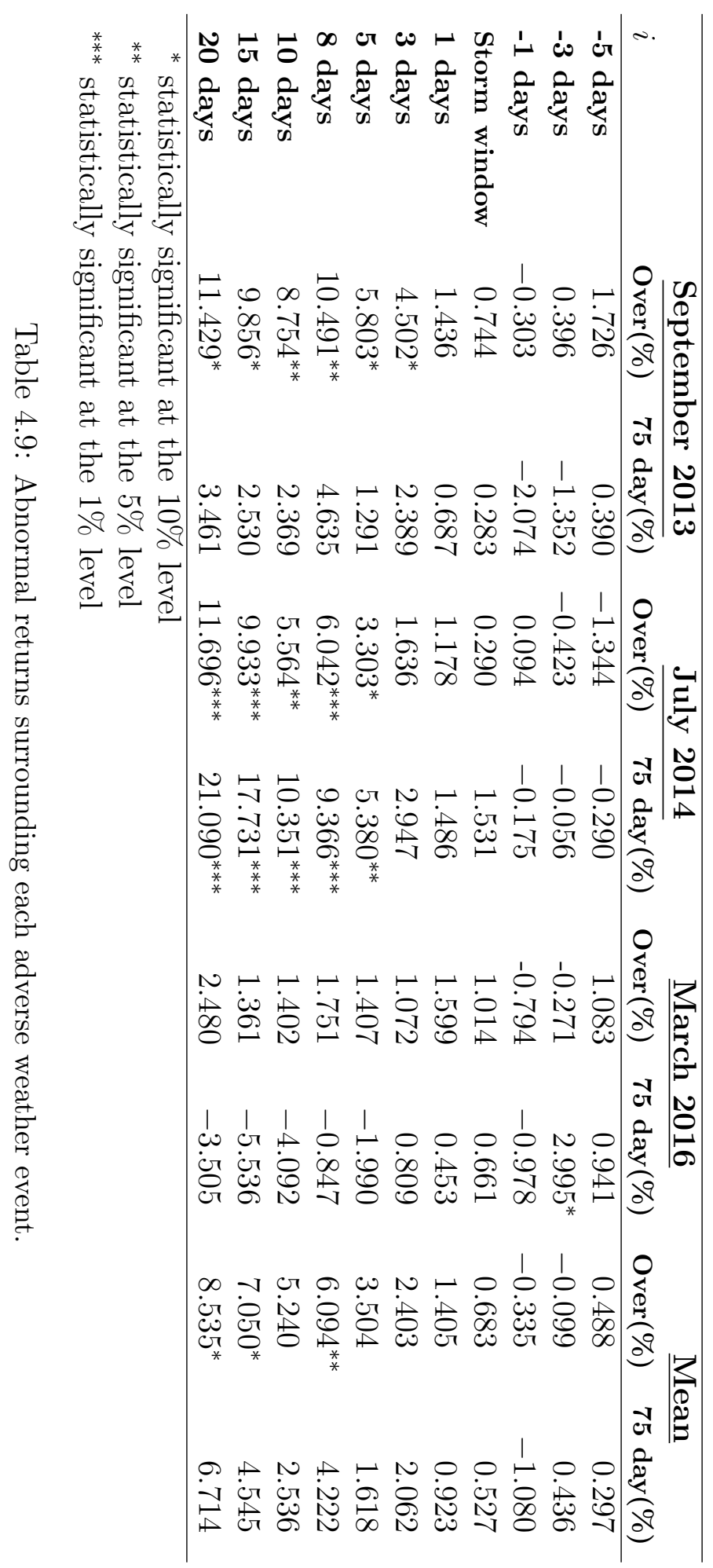




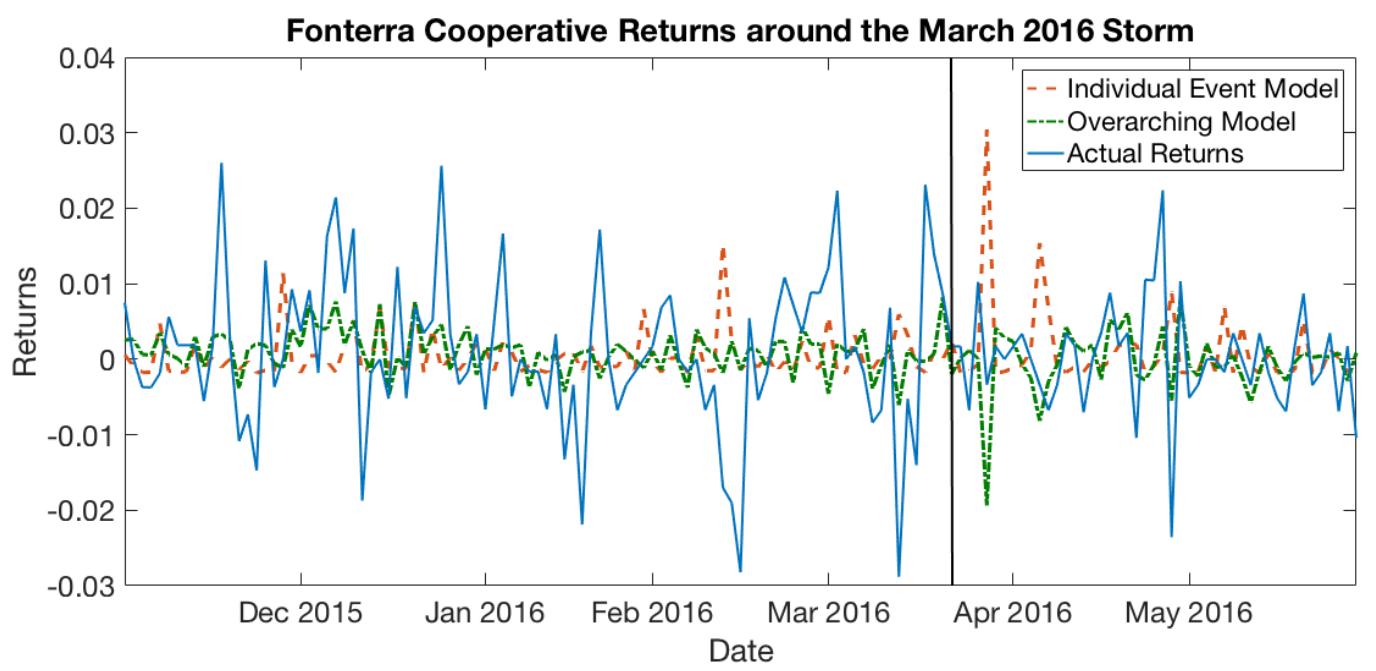

Figure 4.6: Actual FCG returns plotted along with the normal returns model. The vertical black line marks 21 March 2016, the day of the storm.

normal returns (at 5\% significance) for the eight day post-event window. Interestingly the abnormal returns are all positive. The damage was not evenly spread around all dairy farms in the country however. Loss of production in Canterbury may have resulted in increased value for milk that was able to be collected, due to perceived product shortage and demand pressures. Neither of the models showed any significant abnormal returns in the days before the storm arrived, suggesting there was no significant negative reaction to the approaching storm.

We see no significant negative financial impact due to the July 2014 storm, in fact we again see significant positive cumulative abnormal returns in the post-event windows of length eight to twenty days, for both models. For this storm we see larger abnormalities and higher significance with the individual event model. Again we see two large positive spikes following the end of the storm. In this case both spikes show individual significance. The cumulative abnormal returns remain significant to the twenty day post-event window. Again the storms damage was focussed on one area of the country, Northland in this case, but with some farms needing months to recover. ${ }^{5}$ We may be seeing such a significant positive reaction in share price again because of restricted production increasing product value. There are also no significant abnormal returns in the five days before the storm, suggesting no preemption

\footnotetext{
${ }^{5} \mathrm{July}$ is still off season in terms of milk production, but production is beginning to pick up.
} 
of any damage it might bring.

As suggested by the plot in Figure 4.6, there are no significant cumulative abnormal returns for any of the event windows up to twenty days after the March 2016 storm for either the overarching model or the individual event model. We do see positive cumulative abnormal returns, significant at the $10 \%$ level, for three days before the storm hit when using the individual event model. This aligns with a spike seen in Figure 4.6. It is possible there was some sort of preemptive reaction to the storm coming, but the low significance of the reaction and the lack of reaction following the storm suggest the storm did not have much financial affect overall. This storm only lasted one day, potentially reducing the effect. Also while Northland was worst hit in this event, most of the country and dairy producing regions were affected. The shorter length of the storm, together with its relatively even impact across dairying regions may explain why we see little to no impact on share price around this event.

When we look at the average cumulative normal returns over all three adverse weather events we see $5 \%$ significance in the 8 day post-event window, when using the overarching model. This coincides with the beginning of significant cumulative abnormal returns following the September 2013 storm and July 2014 storm. We also see $10 \%$ significance for the cumulative abnormal returns in the 15 and 20 day post-event windows. This suggests we may see some consistent positive reaction building following an adverse weather event, particularly when resulting damage and production loss is confined to specific regions. The loss of production in one area of the country is potentially creating a supply shortage and increasing product value and therefore increasing share price as well. Storms that bring flooding and damage to one part of the country will also likely provide some less intense rainfall to other areas, increasing pasture growth and production in these areas.

\subsubsection{Russian Ban of European Union Farm imports}

The Russian ban on farm imports, from Europe, America and other countries, came into effect on 7 August 2014. The model specification for this event is

$$
\widehat{R}_{t}=\alpha+\beta_{1} \Delta \mathrm{PHP}_{t}+\beta_{2} \Delta \mathrm{ASD}_{t}+\beta_{3}(\Delta \mathrm{USD})^{2}
$$




$$
+\beta_{4}(\Delta \mathrm{JPY})^{2}+\beta_{5}(\Delta \mathrm{CLP})^{2}+\varepsilon_{t}
$$

The individual event model parameters, estimated using the seventy five days preceding the pre-event window are shown in Table 4.10. The proportional

\begin{tabular}{lcc}
\hline Variable & $\begin{array}{c}\text { Parameter } \\
\beta_{i}\end{array}$ & $\begin{array}{c}\text { Standard } \\
\text { Error }\end{array}$ \\
\hline$\Delta \mathbf{P H P}$ & $-0.29871^{* *}$ & 0.14367 \\
$\Delta \mathbf{A S D}$ & $-0.29112^{*}$ & 0.16797 \\
$(\Delta \mathbf{U S D})^{2}$ & $-66.67156^{* *}$ & 29.48191 \\
$(\Delta \mathbf{J P Y})^{2}$ & $116.92242^{* *}$ & 45.86996 \\
$(\Delta \mathbf{C L P})^{2}$ & $-14.74997^{*}$ & 8.25960 \\
Intercept & -0.00051 & 0.00079 \\
\hline$R^{2}$ & 0.202 \\
\hline$n=75$ & \\
${ }^{*}$ statistically significant at the $10 \%$ level \\
${ }^{* *}$ statistically significant at the $5 \%$ level
\end{tabular}

Table 4.10: The individual event normal returns model for the Russian ban on European farm imports

change in the Philippine peso, along with the square of the proportional, changes in the American dollar and Japanese yen are all significant estimators at the $5 \%$ level. As seen in Table 4.1, the Philippines received the fourth highest value of New Zealand dairy exports for the years ending June 2013 and 2014. The proportional change in the Australian dollar is significant at the $10 \%$ level. Australia consistently receives a large proportion of the cheese and curd products exported from New Zealand, second in value only to Japan. The square of the proportional change in the Chilean peso is also significant at the 10\% level in the model. The year ending June 2014 saw the highest annual value of New Zealand dairy exports to Chile for the period cover in our data. Chile also received the fifth highest value of the cheese and curd products exported in that year. Because this was a different type of shock, we have not accounted for the storm that affected New Zealand in July 2014. There is potential this may affect the fit of the model. The presence of heteroskedasticity is not detected by White's test, therefore we do not use HAC standard errors White (1980). 
Figure 4.7 plots the predicted normal returns, as estimated by the model in Table 4.10 and by the 'overarching' model from Table 4.5, for the Russian ban on European farm imports alongside the actual returns. There does not

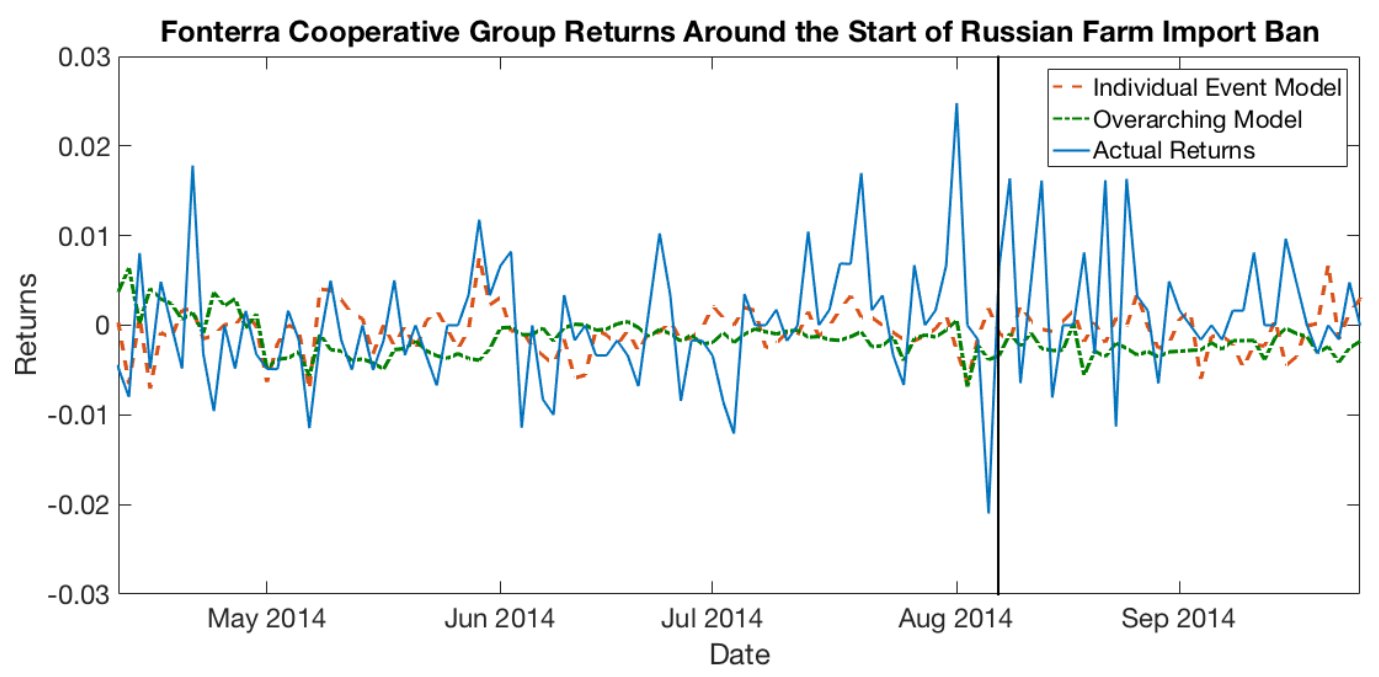

Figure 4.7: Actual FCG returns plotted along with the normal returns model. The vertical black line marks 7 August 2014, the day the import ban came into effect.

appear to be any negative impact on share price returns, in comparison with either model, around the date the Russian farm import ban came into effect, though there may potentially be a small positive effect cumulatively.

The value of cumulative abnormal returns for specific time windows are given in Table 4.11. For statistically significant cumulative abnormal returns we can reject the null hypothesis and accept that they are statistically distinguishable from zero. Both models show significant abnormal returns of about $2 \%$ the day before the Russian ban on European farm imports came into effect, at $5 \%$ and $1 \%$ significance for the overarching and individual event models respectively. In the case of the individual event model, the cumulative abnormal returns are significant at the $5 \%$ level from three days before the ban. Following the ban we begin to see a positive effect of share price returns. For the overarching model this begins the day after the ban with a $2 \%$ increase (5\% significance), reaching a $9 \%$ cumulative increase in returns for the twenty day post-event window, significant at the $1 \%$ level. In the case of the individual event model we see significant cumulative abnormal returns from the day after the beginning of the ban. We observe an increase in FCG returns of almost $2 \%$ one day after the ban was implemented. This increases 


\begin{tabular}{lcc}
\hline & \multicolumn{2}{c}{ Russia Ban } \\
$i$ & Over(\%) & $\mathbf{7 5}$ day $(\%)$ \\
\hline $\mathbf{- 5}$ day & 2.175 & 1.150 \\
$\mathbf{- 3}$ day & -0.968 & $-2.011^{* *}$ \\
$\mathbf{- 1}$ day & $-1.716^{* *}$ & $-2.211^{* * *}$ \\
Event Window & 0.999 & 0.710 \\
$\mathbf{1}$ day & $1.732^{* *}$ & $1.832^{* * *}$ \\
$\mathbf{3}$ day & 1.892 & $1.615^{*}$ \\
$\mathbf{5}$ days & $3.242^{*}$ & $2.484^{* *}$ \\
$\mathbf{1 0}$ days & $6.802^{* * *}$ & $4.934^{* * *}$ \\
$\mathbf{1 5}$ days & $8.580^{* * *}$ & $5.281^{* *}$ \\
$\mathbf{2 0}$ days & $9.673^{* * *}$ & $6.272^{* *}$ \\
\hline${ }^{*}$ statistically significant at the $10 \%$ level \\
${ }^{* *}$ statistically significant at the $5 \%$ level \\
${ }^{* * *}$ statistically significant at the $1 \%$ level
\end{tabular}

Table 4.11: Cumulative abnormal returns surrounding the implementation of the Russian Ban on farm imports.

to a $6.2 \%$ increase, cumulative over twenty days, following the implementation of the ban. It appears that there may have been negative effects in anticipation of the ban, followed by a positive impacts, potentially due to New Zealand not being part of the ban. The ban was announced with one days's notice, which coincides with when we see the most significant negative abnormal returns. It was initially expected that this ban would have a negative effect due to an excess of European product in the global dairy market, we see only a positive reaction however. While the overarching model does control for global dairy price, through the WMP price index, though a surplus in other products could have had an effect. Potentially what we saw instead was expansion into a new market. In 2014, New Zealand exported $\$ 94$ million worth of butter and dairy spreads to Russia (Hutching, 2015), down from $\$ 119$ million in 2013 , but up from $\$ 84$ million 2012. Exports to Russia were negatively affected by bans following the whey protein contamination incident in August 2013, potentially explaining why we see a drop in exports in 2014. Exports to Russia continued dropping to $\$ 31$ million in 2015 , but increased back to $\$ 104$ million in 2016 after these bans were partially lifted (Holloway, 2017). 
The cumulative abnormal returns surrounding Russia's ban on European farm imports, using the 'overarching' model are shown in Table 4.11. We see significant negative abnormal returns on the day before the ban came into effect, followed by significant positive abnormal returns the day after. The cumulative abnormal returns then remain insignificant until the ten day post-event window, where we see significant positive cumulative abnormal returns for the reminder of the analysed event windows.

\subsubsection{End of European Union Milk Quotas}

Milk quotas, restricting the production of dairy farmers in the European Union, were removed on March 31 2015. This event was known about well in advance. Because of this it is not an ideal candidate for an event study. Keeping this caveat in mind we analyse it here regardless. Useful insights still arise from this investigation, but caution is necessary. The specification for this model is

$$
\begin{aligned}
\widehat{R}_{t}= & \alpha+\beta_{1} \Delta \mathrm{CNY}_{t}+\beta_{2} \Delta \mathrm{LKR}_{t}+\beta_{3}(\Delta \mathrm{NZX} 0)^{2} \\
& +\beta_{4}(\Delta \text { Brent Oil })^{2}+\beta_{5}(\Delta \mathrm{EUR})^{2}+\varepsilon_{t}
\end{aligned}
$$

Table 4.12 shows the linear regression model parameters estimated from the data in the seventy five days leading up to the removal of the production caps in the European Union. For this portion of the data the relative change in the Chinese yuan and the Sri Lankan rupee exchange rate are significant at the $5 \%$ level. We also see significance in the squares of the NZX50 returns and the proportional change in the Brent oil price at the $10 \%$ significance level. The square of the change in the exchange rate with the Euro is significant at the $1 \%$ level for this section of the data. White's test does not detect the presence of heteroskedasticity, so we do not use HAC standard errors (White, 1980).

The returns, as estimated by this individual event model and the overarching model from Section 4.4.1, are plotted along with the actual returns in Figure 4.8. There is a large drop in the actual returns several days before the removal of the production caps. This drop coincides with a disappointing farm-gate milk price and dividend announcement on March 25 2015. The milk price 


\begin{tabular}{lcc}
\hline Variable & $\begin{array}{c}\text { Parameter } \\
\beta_{i}\end{array}$ & $\begin{array}{c}\text { Standard } \\
\text { Error }\end{array}$ \\
\hline$\Delta$ CNY & $-0.92407^{* *}$ & 0.43583 \\
$\Delta$ LKR & $1.08685^{* *}$ & 0.43100 \\
$(\Delta \text { NZX50 })^{2}$ & $27.23450^{*}$ & 13.66703 \\
$(\Delta \text { Brent oil })^{2}$ & $1.10237^{*}$ & 0.55762 \\
$(\Delta \mathbf{E U R})^{2}$ & $-18.32402^{* *}$ & 6.59461 \\
Intercept & -0.00037 & 0.00103 \\
\hline$R^{2}$ & 0.25 \\
\hline$n=75$ & \\
${ }^{*}$ statistically significant at the $10 \%$ level \\
${ }^{* *}$ statistically significant at the $5 \%$ level \\
${ }^{* * *}$ statistically significant at the $1 \%$ level
\end{tabular}

Table 4.12: Parameters of the seventy five day normal returns model leading up tot the removal of milk quotas in the European Union

forecast remained at $\$ 4.70$ per $\mathrm{kg}$ MS, but the interim dividend of ten cents was lower than expected. The forecast dividend of just twenty - thirty cents per share was a five cent drop from the previous forecast (Rotherham, 2015). Kilsby (2015) suggests the sudden drop in share price was a response to the reduced dividend forecast. In the days following the end of the quota system it appears there may be a drop in returns overall. For approximately two weeks following the removal of the production caps, the actual returns sit either on or below the returns as predicted by the overarching model. This drop does not affect the estimation or either of the models as it is within the five days preceding the cap removal. Neither of our models predict this large drop in share price either. It appears there may be a slightly delayed drop in returns from about a week after the event.

The cumulative abnormal returns surrounding the end of the dairy production quota system, using both the overarching model and the individual event model, are presented in Table 4.13. Again, significant abnormal returns are statistically distinguishable from zero. We see significant cumulative abnormal returns in the each of the pre-event window lengths, likely due to the large drop as discussed previously. The link to disappointing process and dividends means we cannot accurately conclude any affect from the European Union cap removal here. On the day the caps were removed we see significant abnormal returns, at the $5 \%$ level, for the overarching model, but only at the 


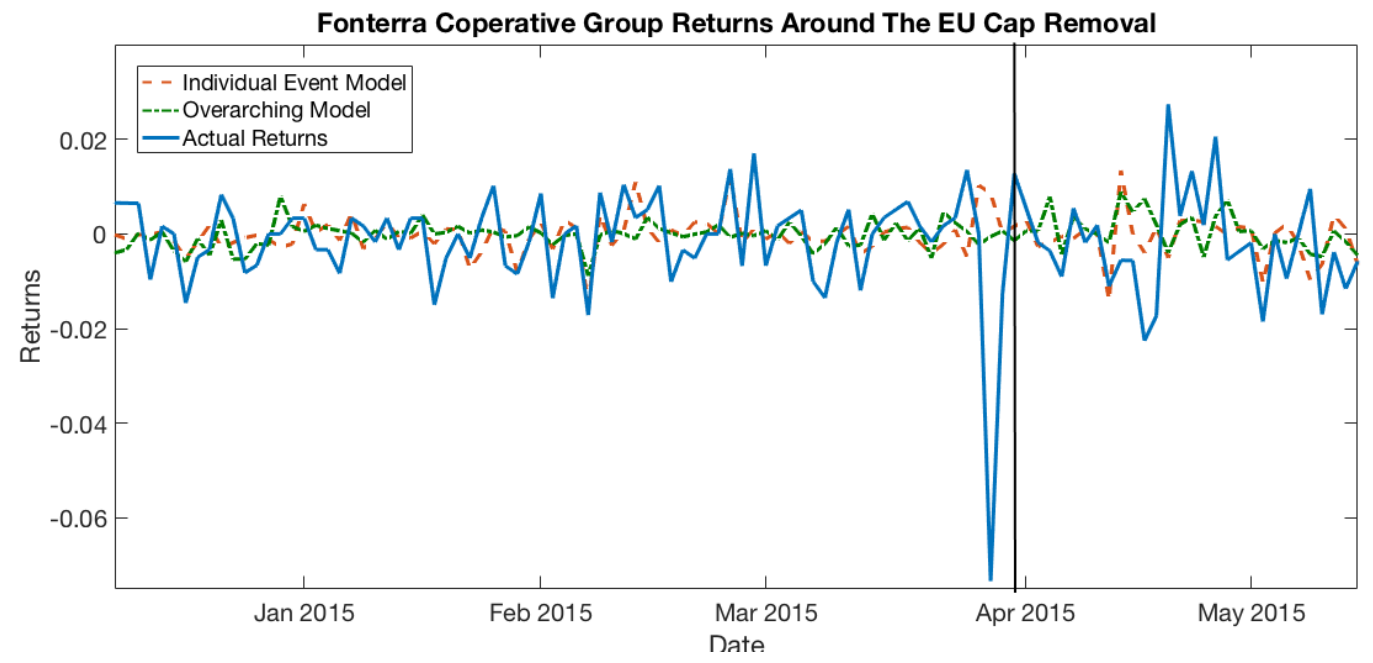

Figure 4.8: Actual FCG returns plotted along with the returns predicted by each model. The vertical black line marks 31 March 2015, the date the quota system ended.

\begin{tabular}{lcc}
\hline & \multicolumn{2}{c}{ EU Quotas } \\
\cline { 2 - 2 }$(\mathbf{6}$ days & $\mathbf{7 5}$ day $(\%)$ \\
\hline $\mathbf{- 3}$ days & $-5.557^{* * *}$ & $-8.421^{* * *}$ \\
$\mathbf{- 1}$ day & $-6.960^{* * *}$ & $-10.505^{* * *}$ \\
Event Window & $-1.322^{* *}$ & $-1.284^{* *}$ \\
$\mathbf{1}$ day & $1.417^{* *}$ & $1.115^{*}$ \\
$\mathbf{3}$ days & 0.474 & 0.254 \\
$\mathbf{5}$ days & -0.909 & 0.158 \\
$\mathbf{1 0}$ days & -1.199 & -0.054 \\
$\mathbf{1 5}$ days & $-4.689^{* *}$ & -2.344 \\
$\mathbf{2 0}$ days & $-5.324^{* *}$ & -1.683 \\
${ }^{*}$ statistically significant at the $10 \%$ level \\
${ }^{* *}$ statistically significant at the $5 \%$ level \\
${ }^{* * *}$ statistically significant at the $1 \%$ level
\end{tabular}

Table 4.13: Cumulative abnormal returns surrounding the removal of dairy production quotas in the EU.

$10 \%$ level for the individual event model. The day after the removal of the production caps we see no significant abnormal returns. For up to five days following the cap removals the cumulative abnormal returns are not significantly different from zero for either model. We do not see any significant cumulative abnormal returns for any of the post-event windows when using the individual event model. From ten days after the cap removal however, 
and for the fifteen day post-event window, we see reduced cumulative abnormal returns significant at the $5 \%$ level, using the overarching model. This is potentially a delayed reaction, possibly due to surplus production taking time to build up and have an effect on global demand for dairy. This what we expected to see, but the lack of significance for the individual event model suggests the parameters in this model may be affected by the same event. This model does have a significant negative relationship with the squared change in the Euro exchange rate.

\subsection{Discussion and Conclusions}

In this chapter we have explored the financial impact of several shocks to the New Zealand dairy industry. Each shock is unique and causes a unique reaction in the share price returns. We are able to identify some patterns in these reactions however.

Though it is not always significant, the first five days following each recall show negative cumulative abnormal returns. Greater media coverage has been associated with a larger negative reaction in share price following a recall (Pozo \& Schroeder, 2015), however out of the recalls we analysed, the largest impact was seen following the Mainland cheese recall, that with the least coverage. This recall did involve exported product, though only to Fiji, which is a relatively small market for Fonterra. Annual dairy exports to Fiji typically comprise around $0.3 \%$ of total dairy exports. Dairy exports to Fiji in June 2013 were unusually low however. Only $\$ 1.4$ million in dairy exports we sent to Fiji June 2013, compared to an average of $\$ 3.5$ million for June over the other years in our time frame (Statistics New Zealand, 2017b). This was the first recall by Fonterra following the implementation of the new financial structure in December 2012, so this recall reaction may reflect a lack of experience from Fonterra and its shareholders regarding how share price returns may now be vulnerable to such events. Both the WPC80 contamination incident and the Pams/Anchor recall showed relatively small negative reactions, these were also not consistent between the models used in each case. This is consistent with the finding in Pozo \& Schroeder (2015), that recurrent recalls have less impact the a firm's first recall. 
In analysing the impacts of adverse weather events, we found a somewhat unexpected positive impact on share price returns. From the events analysed it appears that this positive effect is diminished as more areas are affected. The July 2014 storm, which showed the largest impact, affected the smallest area, causing significant damage in the Northland region only. This storm also lasted the longest of the three. It is possible that loss of production in one area of the country only is increasing perceived value of what is produced. Rain also usually comes with storms, and in each of these storms most of the country saw some rain, so there may be an effect of anticipated production due to improved pasture growth.

The Russian ban on farm imports from selected countries showed a negative impact on share price in the days before it came into effect, particularly for the day it was announced. In the days after the ban was implemented however we start to see a positive financial impact. A surplus of global supply may have been anticipated, particularly due to a large amount of European product no long being exported to Russia and needing somewhere else to go. The positive reaction following the ban may reflect that the surplus was perhaps not as large as anticipated. At the next GDT auction, the SMP price index dropped $12 \%$. None of the other product indices dropped at all and overall the GDT price index dropped by just $0.6 \%$. All of the price indices did drop at the next auction, resulting in a drop of $6 \%$ for the GDT price index. Prices started to come back up from the auction on 15 October 2014, and the AMF and butter price indices were back to pre event levels by November 2014. The SMP price index has remained relatively low, but the GDT price index was back to pre-event levels by February 2015. A potential space in the market, due to New Zealand being exempt from the ban, may also have been perceived by share holders, as Russia was forced to seek out new trading partners (Khachaturyan et al., 2017).

We found a significant negative impact on share price returns in the days before the end of the European Union dairy production quota system. Increasing dairy production in Europe meant an increase in global supply, not matched by an increase in demand, causing a surplus in the global market. The initial surplus may not have been as large as anticipated, resulting in the lack of significance in cumulative abnormal returns in the post-event windows. We do see some significance in comparison with the full overarching 
model for the ten and fifteen day post-event windows.

Because of the limited time Fonterra has been operating under the current financial structure, we are limited in the number of events available for analysis. A broader study of each event type would help to identify patterns and important characteristics of each event with more certainty. As mentioned in Section 4.1.2, we have not looked at FSF units traded on the Australian stock exchange, nor specifically used the price of any FSF units in our analysis. The correlation coefficient between the Australian traded units and the FCG shares is only 0.5863. Conducting similar analyses on these events using these related stocks therefore may help to strengthen results and provide clearer insights. We also briefly discuss the potential for using a Fonterra specific GDT index in Section 4.A, this is something that could be a useful variable for future research in this area.

There are some limitations in finding significant impact due to the generally low R-squared values suggesting poor fit of the models. As mentioned in Sections 4.3.3 and 4.4.1, this is taken into account in the calculation of the test statistic, but may mean some significant impacts go undetected. Care is needed to avoid over fitting models, however an investigation into the use of other types of models, such as GARCH (mentioned in Section 4.1.1), may help to improve the analysis.

Overall the greatest potential for a negative financial impact comes from food contamination and recall events. Weather events and international events impacting global supply are seen as outside of a company's control. In the case of a recall someone can be held accountable. A recall event is a negative reflection on a firm's quality and safety assurance systems. An error has been made somewhere in the production process, and for some unknown reason was not rectified before product could reach consumers. There is a sense of hidden information in the circumstances leading to the recall. This leads to an added reputation effect in the case of contamination and recalls that may not be applicable to with other type of shocks. A study looking particularly at recall type events and how reputation may have been affected along with share price reactions could provide more insight into the importance of handling recalls well. 


\section{Appendix 4.A GDT Price Index}

The GDT price index is a weighted average of the percentage change in prices. Specifically it is a chain-linked Fisher index (GDT, 2016b), that is the geometric mean of the Laspenyres and Paasche indices (Statistical Office of the European Communities, 2009). The weights are determined by the quantity of product sold in each contract at each trading event (GDT, 2016 b). Let $P$ be a $2 \times n$ matrix of the prices at two consecutive trading events, with Row one representing the prices at the first event, and row two the prices at the second. Similarly let $W$ be a $2 \times n$ matrix of the quantity of product sold for each of the prices in $P$. Now let $M=P W^{T}$, the $2 \times 2$ matrix of weighted prices. The Laspenyres, Paasche and Fisher price indices are then given by:

$$
\begin{aligned}
\text { Laspeyres } & =\frac{m_{21}}{m_{11}} \\
\text { Paasche } & =\frac{m_{22}}{m_{12}} \\
\text { Fisher } & =\sqrt{\frac{m_{21}}{m_{11}} \times \frac{m_{22}}{m_{12}}}
\end{aligned}
$$

Constructing a Fonterra specific GDT index was considered, while some traded volumes are freely available from AgriHQ (2016), the contract prices were not obtainable for this thesis. There are also many sub products for each product type that make estimating such an index impractical within the scope of this thesis.

\section{Appendix 4.B Complete Overarching Model}

The complete overarching model from Section 4.4.1 is presented in Table 4.14 


\begin{tabular}{|c|c|c|c|}
\hline Variables & Month & Parameter & Std Error \\
\hline$\Delta$ NZX50 & - & $-0.10517^{*}$ & 0.06528 \\
\hline$(\Delta \text { Production })^{2}$ & - & $0.04313^{* *}$ & 0.02331 \\
\hline$(\mathrm{WMP})^{2}$ & - & $-0.00142^{* *}$ & 0.00070 \\
\hline$(\Delta \mathrm{CNY})^{2}$ & - & $12.14775^{*}$ & 6.37328 \\
\hline$(\Delta \mathbf{L K R})^{2}$ & - & $-19.39685^{* * *}$ & 7.28947 \\
\hline \multirow{11}{*}{$\Delta$ Production } & February & 0.00387 & 0.01495 \\
\hline & March & -0.00373 & 0.01085 \\
\hline & April & -0.00508 & 0.00754 \\
\hline & May & $-0.02314^{* *}$ & 0.01010 \\
\hline & June & -0.01157 & 0.01538 \\
\hline & July & $-0.02919^{* * *}$ & 0.00699 \\
\hline & August & -0.05758 & 0.06086 \\
\hline & September & $-0.05144^{*}$ & 0.03924 \\
\hline & October & 0.01092 & 0.01341 \\
\hline & November & -0.01812 & 0.01228 \\
\hline & December & $-0.07980^{* *}$ & 0.04096 \\
\hline \multirow{11}{*}{$\Delta$ Brent Oil } & February & 0.00963 & 0.01869 \\
\hline & March & -0.01089 & 0.04631 \\
\hline & April & 0.05346 & 0.22217 \\
\hline & May & -0.01815 & 0.04920 \\
\hline & June & 0.09484 & 0.07101 \\
\hline & July & -0.06542 & 0.06438 \\
\hline & August & $-0.10258^{* *}$ & 0.04886 \\
\hline & September & 0.01222 & 0.04669 \\
\hline & October & 0.00502 & 0.05578 \\
\hline & November & 0.00575 & 0.03055 \\
\hline & December & -0.06658 & 0.04150 \\
\hline \multirow{11}{*}{$\Delta \mathbf{U S D}$} & February & -0.09369 & 0.09436 \\
\hline & March & 0.19015 & 0.13891 \\
\hline & April & $0.41861^{*}$ & 0.27447 \\
\hline & May & -0.11577 & 0.13685 \\
\hline & June & -0.04781 & 0.10063 \\
\hline & July & 0.06349 & 0.10248 \\
\hline & August & -0.00981 & 0.12186 \\
\hline & September & 0.03655 & 0.14783 \\
\hline & October & 0.01450 & 0.11233 \\
\hline & November & $-0.18502^{* *}$ & 0.08468 \\
\hline & December & $0.26360^{* *}$ & 0.12272 \\
\hline \multirow{11}{*}{$\Delta \mathbf{E U R}$} & February & 0.20723 & 0.14554 \\
\hline & March & -0.08567 & 0.13413 \\
\hline & April & 0.03667 & 0.50992 \\
\hline & May & 0.11538 & 0.12539 \\
\hline & June & 0.11668 & 0.16994 \\
\hline & July & 0.18263 & 0.11329 \\
\hline & August & 0.27136 & 0.44667 \\
\hline & September & 0.09790 & 0.15047 \\
\hline & October & 0.00370 & 0.13763 \\
\hline & November & $0.17323^{*}$ & 0.09956 \\
\hline & December & $0.39557^{* *}$ & 0.17299 \\
\hline Intercept & - & $0.00193^{* *}$ & 0.00083 \\
\hline$R^{2}$ & & 0.095 & \\
\hline \multicolumn{4}{|l|}{$\begin{array}{l}n=801 \\
\quad * \text { statistically sign }\end{array}$} \\
\hline \multicolumn{4}{|c|}{${ }^{* *}$ statistically significant at the $5 \%$ level } \\
\hline
\end{tabular}

Table 4.14: All parameter values for the normal returns for the overarching model. 


\section{Chapter 5}

\section{Summary and Conclusions}

This thesis covers various aspects of the dairy industry in New Zealand, highlighting areas where improvements might be made to increase overall output and reduce the impact of shocks.

In Chapter 2 of this thesis we develop a discrete time Markov chain model for several stages of a dairy supply chain. We model the flow of milk, in terms of its value, from the farm through to the first stage of processing common to all dairy products. This model is applied specifically to the case of Fonterra, New Zealand's dominant dairy co-operative, allowing for costs associated with testing and resulting product rejections. By running simulations under various testing scenarios, we are able to identify where and when rejection of product can cause the greatest reduction in the overall value produced. We find the probability of rejecting product as milk tankers deliver to the factory has the greatest effect on production rates. Overall output value ranged between over $\$ 26$ million with no rejection at the factory reception stage, down to $\$ 20$ million with a $75 \%$ acceptance rate. The rate of rejection at the tanker collection stage was shown to have the least impact, out of the three stages modelled, with overall output value ranging between $\$ 26$ million with no rejection and $\$ 22.5$ million when acceptance rates for this stage were only $75 \%$. If by being more strict about removing contaminated product at the point of tanker collection, product loss at the factory reception stage is able to be significantly reduced, this is an approach that would be useful in increasing the overall output value of the supply chain.

The model developed in Chapter 2 is extended to include elements of trace- 
ability in Chapter 3. Simulations under various scenarios, including different levels of traceability at different stages of the supply chain, allow us to gain an understanding of where and when traceability has the greatest impact. For each of these scenarios we are able to estimate the value traceability contributes to the supply chain. There is significant value to be gained through using traceability insights to precisely identify faulty product for removal when it results in less primary rejections than via test results alone. The largest reduction in value lost is seen when traceability is applied across all stages of the supply chain. Upon exclusive application in individual stages however, the greatest benefit is seen when traceability information is collected as the tanker collects milk from the farm, resulting in reduced primary rejection upon application of that information when the tanker delivers milk to the factory reception stage. The potential for improvement in output value decreases as the initial acceptance rate analysed increases. For example, with an initial $75 \%$ acceptance rate, when traceability information is not applied until the processing stage, we see a potential improvement in overall output value of $9.4 \%$. If traceability information can be applied only at the factory reception stage we see a potential improvement of $11.9 \%$. When traceability is applied in both of these stages though, we see an average improvement of $27 \%$ in overall production value. We this see an effect here where the whole is greater the the sum of the parts. The potential value to be gained in the application of a good traceability system is greater when applied over all of the supply chain than just the sum of the individual potential gains we see at each stage.

The are some limitations to be aware of regarding the modelling done in Chapters 2 and 3. Firstly we must remember that the models developed are still just that. No model can perfectly replicate reality, to quote the statistician George Box "All models are wrong; but some are useful" (Box, 1979). We model the individual compartments in each stage of production as a pool, which value enters and exits at appropriate rates in appropriate volumes. A more accurate model may be achievable by modelling individual compartments separately, however this would make it more challenging to apply the model to other industries and supply chains as desired. Because of this, our models also do not take into account inter-site transfers that may take place within the same stage of processing. Another area to be investigated is po- 
tential extra costs associated with more testing and traceability happening earlier on in the supply chain. Our model has identified a value for traceability, but for a given industry or company, the costs of implementation may outweigh the benefits. In the case of Fonterra, these costs are relatively small compared to the value of milk the company processes and distributes, however this may not be the case for smaller companies. The model, presented through these chapters, also does not include the costs of lost goodwill and reputation, focusing solely on the cost of product loss and reducing this. If we were to include reputation effects, the value of traceability may in fact be even greater.

The model we have developed through Chapters 2 and 3, is also a useful tool for theoretically assessing the value of traceability in a supply chain. While the model is applied specifically to dairy production for the purposes of this research, the method is also applicable to other production industries. By choosing parameters appropriate to the industry in question many applications are possible. While traceability is important for food safety and reducing the potential impact of contamination events, it can also aid in the optimisation of production planning and schedules, creating a competitive advantage. This model could be used to assess the impact of processing with different batch sizes. With accurate information regarding the cost associated with each batch processed at each stage, a cost benefit approach could be used to find the optimal batch size under given conditions. Better knowledge about the path product and ingredients take can help to identify redundancies and streamline the supply chain.

A different aspect of the dairy industry is investigated in Chapter 4, specifically how share prices react to various supply and demand shocks. An event study technique is used to analyse how share price returns behave around various event types, including recalls, adverse weather events and global supply shocks. We see limited consistency in reactions to recalls, though the reaction is generally negative and the largest negative reaction out of all the events analysed followed a recall event. The adverse weather events consistently followed abnormally positive share price returns, potentially reflecting Fonterra's influence in the global dairy market. If there is a shortage in milk production the milk that is available becomes worth more, mitigating any negative effects on share prices. The ban on farm imports implemented by 
Russia in response to the sanctions placed on their exports was also followed by abnormally positive share prices returns. The expected global surplus due to European dairy product having lost a major market drove down milk price, thus a negative reaction in share price was expected. The fact that Russia still needed to import dairy products potentially made New Zealand milk valuable enough to counteract the surplus effect. Annual dairy exports to Russia did drop in value from 2013 to 2014 but remained higher than in 2012. It is difficult to conclude that this may be the reason for positive abnormal returns however. The removal of dairy production caps in the European Union, while not an ideal occurrence for an event study, was followed by abnormally negative share price returns as expected, potentially due to an increased surplus in global milk supply.

The conclusions we can draw are limited by the nature of event studies. Again we are using models to imitate reality, though in this case our models generally have low $R^{2}$ values, indicating poor fit. The fit of the model is taken into account in the test statistics, reducing the likelihood of any type I errors, where we attribute significance to abnormal returns that in reality are not. Poor fit will however increase the potential for Type II errors, meaning we might dismiss some significant reactions as insignificant. An event study is also really an analysis of what is happening to the share price around the time of the event, we cannot with certainty attribute the movements in the share price to the specific event. The fact the the largest negative reaction in share price returns followed a recall event does however highlight the importance of managing recalls well. All of the results in this thesis suggest product recalls are something to be taken seriously, particularly in the dairy industry. Firms should be well prepared to react swiftly and efficiently to minimise the impact when such events do occur. 


\section{References}

Akkerman, R., Farahani, P., \& Grunow, M. (2010). Quality, safety and sustainability in food distribution: a review of quantitative operations management approaches and challenges. OR Spectrum, 32(4), 863-904.

Allen, L. J., Brauer, F., Van den Driessche, P., \& Wu, J. (2008). Mathematical epidemiology. Springer.

Alltech. (2017). Playing fields leveled as EU milk quotas are abolished after 30 years. Retrieved 17/04/17, from http://ag.alltech.com/en/blog/ playing-fields-leveled-eu-milk-quotas-are-abolished-after-30 -years

Alves, H., Canadas, N., \& Rodrigues, A. M. (2015). Determinants of share price and share liquidity: An analysis using a sem model. Procedia Economics and Finance, 25, 318-331.

Anderson, C. (2013, 17 September). Milk rejected in power cut crisis. Retrieved 15/04/17, from http://www.stuff.co.nz/the-press/news/ canterbury/9172307/Milk-rejected-in-power-cut-crisis

Ballingall, J., \& Pambudi, D. (2017). Dairy trades economic contribution to New Zealand (Tech. Rep.). New Zealand Institute of Economic Research.

Beliën, J., \& Forcé, H. (2012). Supply chain management of blood products: A literature review. European Journal of Operational Research, 217(1), $1-16$.

Bilgen, B., \& Dogan, K. (2015). Multistage production planning in the dairy industry: a mixed-integer programming approach. Industrial \& Engineering Chemistry Research, 54(46), 11709-11719.

Biswas, S., \& Narahari, Y. (2004). Object oriented modeling and decision support for supply chains. European Journal of Operational Research, $153(3), 704-726$.

Boland, V. (2015, 31 March). Irish dairy farmers celebrate end of EU milk quotas. In Financial Times. 
Bollen, A., Riden, C., \& Cox, N. (2007). Agricultural supply system traceability, part i: Role of packing procedures and effects of fruit mixing. Biosystems engineering, 98(4), 391-400.

Boppana, R. V., \& Chalasani, S. (2007). Analytical models to determine desirable blood acquisition rates. In System of systems engineering, $200 \%$. sose'07. ieee international conference on (pp. 1-6).

Boulanger, P., Dudu, H., Ferrari, E., \& Philippidis, G. (2015, 17-19 June). The cost of import prohibition for political reason: CGE analysis of the Russian ban on agri-food products. In 18th annual conference on global economic analysis. information for the policy maker: Practical economic modelling for tomorrow. Melbourne, Australia. Retrieved from http:// hdl.handle.net/10532/3114

Box, G. E. (1979). Robustness in the strategy of scientific model building. Robustness in statistics, 1, 201-236.

Brodheim, E., Hirsch, R., \& Prastacos, G. (1976). Setting inventory levels for hospital blood banks. Transfusion, 16(1), 63-70.

Brown, S. J., \& Warner, J. B. (1985). Using daily stock returns: The case of event studies. Journal of financial economics, 14 (1), 3-31.

Buhr, B. L. (2003). Traceability and information technology in the meat supply chain: implications for firm organization and market structure. Journal of Food Distribution Research, 34(3), 13-26.

Campbell, J. Y., Lo, A. W.-C., \& MacKinlay, A. C. (1997). The econometrics of financial markets. princeton University press.

Canavari, M., Centonze, R., Hingley, M., \& Spadoni, R. (2010). Traceability as part of competitive strategy in the fruit supply chain. British Food Journal, 112(2), 171-186.

Carriquiry, M., \& Babcock, B. A. (2007). Reputations, market structure, and the choice of quality assurance systems in the food industry. American Journal of Agricultural Economics, 89(1), 12-23.

Castleton, A. (2016, 10 March). GDT volumes mostly as forecast. Retrieved from https://agrihq.co.nz/section/all/view/gdt-volumes -mostly-as-forecast

Centrico. (1994). Separators for the dairy industry: Operating instructions [Computer software manual]. Northvale, New Jersey: Centrico, Inc.

Chan, E., Griffiths, S., \& Chan, C. (2008). Public-health risks of melamine in milk products. The Lancet, 372(9648), 1444. 
Chand, A. (2006). On-farm fractionation of milk components (Unpublished doctoral dissertation). The University of Waikato.

Comba, L., Belforte, G., Dabbene, F., \& Gay, P. (2013). Methods for traceability in food production processes involving bulk products. Biosystems engineering, 116(1), 51-63.

Dabbene, F., \& Gay, P. (2011). Food traceability systems: Performance evaluation and optimization. Computers and Electronics in Agriculture, $75(1), 139-146$.

Dairy Companies Association of New Zealand. (2017). About the nz dairy industry. Retrieved 26/07/17, from https://www.dcanz.com/about-the -nz-dairy-industry/

Dairy Traceability Working Group. (2014, December). Report A: Proposed regulatory requirements for traceability.

Doganis, P., \& Sarimveis, H. (2007). Optimal scheduling in a yogurt production line based on mixed integer linear programming. Journal of Food Engineering, 80(2), 445-453.

Doganis, P., \& Sarimveis, H. (2008a). Mixed integer linear programming scheduling in the food industry. Optimization in food engineering, 305.

Doganis, P., \& Sarimveis, H. (2008b). Optimal production scheduling for the dairy industry. Annals of Operations Research, 159(1), 315-331.

Dooley, A., Parker, W., \& Blair, H. (2005). Modelling of transport costs and logistics for on-farm milk segregation in new zealand dairying. Computers and electronics in agriculture, 48(2), 75-91.

Dowson, O. (2017). Dairy analytics: Farmgate milk price forecaster. http:// dairyanalytics.co.nz. ([Accessed 11 August 2017])

Draper, S. (2016, 30 September). Sri lanka: PM's visit signals it's open for business. In NBR. Retrieved from https://www.nbr.co.nz/article/ sri-lanka-pm-wickremesinghes-visit-signals-its-open-business -ng-194973

Dupuy, C., Botta-Genoulaz, V., \& Guinet, A. (2005). Batch dispersion model to optimise traceability in food industry. Journal of Food Engineering, 70(3), 333-339.

Etzel, M., Root, T., Arunkumar, A., \& Gemili, S. (2012, February 2). Methods and compositions involving whey protein isolates. Google Patents. Retrieved from http://www.google.co.nz/patents/US20120029165 (US Patent App. 13/181,234) 
European Commission. (2017a). The end of milk quotas. Retrieved 17/04/17, from https://ec.europa.eu/agriculture/milk-quota-end_en

European Commission. (2017b). EU responses to the Russian import ban on agricultural products. Retrieved 16/04/17, from https://ec.europa.eu/ agriculture/russian-import-ban_en

Flegal, J. M., Haran, M., \& Jones, G. L. (2008). Markov chain monte carlo: Can we trust the third significant figure? Statistical Science, 250-260.

Fonterra. (2010, 8 August). New Zealand's biggest spring clean gets fonterra sites in 'peak' condition. Retrieved 08/04/15, from https:// www . infonews. co.nz/news. cfm?id=54695

Fonterra Co-operative Group. (2014a). Fonterra in New Zealand. Retrieved 08/04/15, from http://www.fonterra.com/global/en/About/ Our+Locations/NewZealand/

Fonterra Co-operative Group. (2014b). Fonterra suppliers' handbook 2014/2015. Fonterra Co-operative Group.

Fonterra Co-operative Group. (2015a). Farmgate milk price statement. Retrieved 24/02/16, from https://view.publitas.com/ fonterra/farmgate-milk-price-statement/

Fonterra Co-operative Group. (2015b, 24 September). Media release: Fonterra strenghtens second half performance in 2014/15. Retrieved from https://nzx.com/files/attachments/221347.pdf

Fonterra Co-operative Group. (2016a, 21 January). Paediatric supply chain map. (Personal Correspondence, Tim Kirk)

Fonterra Co-operative Group. (2016b). Shares and units. Retrieved 12/04/17, from https://www.fonterra.com/nz/en/our-financials/ shares-and-units.html

Fonterra Co-operative Group. (2017a, 1 April). Milk price methodology. Retrieved 01/03/17, from https://www.fonterra.com/nz/en/our -financials/farmgate-milk-prices/milk-price-methodology.html

Fonterra Co-operative Group. (2017b). Our markets. Retrieved 18/09/17, from https://www.fonterra.com/nz/en/our-markets.html

Food Standards Australia New Zealand. (2017, January). Food traceability. Retrieved from http://www.foodstandards.govt.nz/industry/ safetystandards/traceability/pages/default.aspx 
Fox, A. (2013, 23 May). Voluntary cheese slice product recall. In Waikato times. Fairfax New Zealand Limited. Retrieved 27/04/17, from http://www.stuff.co.nz/business/farming/dairy/ 8709370/Voluntary-cheese-slice-product-recall

Gao, C. (2011). Stock market response to the 2008 china milk scandal (Unpublished doctoral dissertation). Rutgers The State University of New Jersey-New Brunswick.

GDT. (2016a). GDT Events frequently asked questions. Retrieved 24/07/17, from https://www.globaldairytrade.info/en/gdt-events/ gdt-events-frequently-asked-questions/\#section-8

GDT. (2016b, March). GDT Events: How GDT Price Indicies are calculated. Retrieved 24/7/17, from http://www.globaldairytrade.info/assets/ Uploads/resources/GDT-Price-Index-Briefing-Note-6-1.pdf

Golan, E. H., Krissoff, B., Kuchler, F., Calvin, L., Nelson, K., Price, G., et al. (2004). Traceability in the us food supply: economic theory and industry studies. Washington, DC 20024-3221: US Department of Agriculture, Economic Research Service.

Gorton, M., Dumitrashko, M., \& White, J. (2006). Overcoming supply chain failure in the agri-food sector: A case study from moldova. Food Policy, $31(1), 90-103$.

Gray, J. (2014, 6 September). Russia ban hits NZ exports. In NZ Herald. (http://www.nzherald.co.nz/business/news/article.cfm?c_id= 3\&objectid=11319843)

GS1 New Zealand. (2017). ProductRecallNZ. Retrieved 12/09/17, from http://www.gs1nz.org/services/productrecallnz/

Hanson, K., Robinson, S., \& Schluter, G. (1993). Sectoral effects of a world oil price shock: economy-wide linkages to the agricultural sector. Journal of Agricultural and Resource Economics, 96-116.

Healy, A. (2015, 1 April). Dairy farmers celebrate as milk quotas abolished after 31 years. In The Irish Times.

Hendricks, K. B., \& Singhal, V. R. (2003). The effect of supply chain glitches on shareholder wealth. Journal of operations Management, 21(5), 501-522.

Hogeveen, H., \& Ouweltjes, W. (2002). Sensors and management support in high-technology milking. Journal of animal science, 81, 1-10. 
Holloway, S. (2016, 13 December). EU to pass NZ as world's largest dairy exporter. Retrieved from https://agrihq.co.nz/topic/markets -and-data/exports/china-buying-more-dairy-products

Holloway, S. (2017, 13 February). Russian threats to dairy arise again. Retrieved 06/09/17, from https://agrihq.co.nz/topic/govt -and-legislation/view/russian-threats-to-dairy-arise-again

Horvath, P. A., Autry, C. W., \& Wilcox, W. E. (2005). Liquidity implications of reverse logistics for retailers: A Markov chain approach. Journal of retailing, 81(3), 191-203.

Hudson, A., Wong, T., \& Lake, R. (2003, November). Pasteurisation of dairy products: times, temperatures and evidence for control of pathogens (Tech. Rep.). Christchurch, New Zealand: Institute of Environmental Science and Research, Ltd.

Huffman, L. M., \& Harper, W. J. (1999). Maximizing the value of milk through separation technologies. Journal of dairy science, 82(10), 22382244 .

Hunt, N. (2015, 2 April). End of EU milk quotas raises cheers, and dire warnings. In D. Evans \& W. Hardy (Eds.), Business News (United Kingdom ed.). Reuters.

Hutching, G. (2015, 19 August). Fonterra plants can send milk products to russia. Fairfax New Zealand Limited. Retrieved 25/07/17, from http:// www .stuff .co.nz/business/farming/agribusiness/71277159/ russia-lifts-restrictions-on-new-zealand-milk-imports

Hutchinson, C. A. (2006). Multiscale modelling as an aid to decision making in the dairy industry (Unpublished master's thesis). University of Canterbury, Chemical and Process Engineering, Christchurch, New Zealand.

Interest. (2017). Dairy industry payout history. interest.co.nz. Retrieved 18/09/17, from http://www.interest.co.nz/rural-data/dairy -industry-payout-history

International Dairy Foods Association. (2010, September). An International Comparison of Milk Supply Control Programs and Their Impacts (Tech. Rep.). 3464 Washington Dr. Eagan, MN 55122 USA: Informa Economics, Inc. Retrieved from http://future.aae.wisc.edu/ publications/Informa_Supply_Control_Impacts_0910.pdf.

Jansen-Vullers, M. H., van Dorp, C. A., \& Beulens, A. J. (2003). Managing traceability information in manufacture. International Journal of Information Management, 23(5), 395-413. 
Jiang, N., \& Sharp, B. (2014). Cost efficiency of dairy farming in new zealand: a stochastic frontier analysis. Agricultural and Resource Economics Review, 43(3), 406-418.

Karlsen, K. M., Dreyer, B., Olsen, P., \& Elvevoll, E. O. (2013). Literature review: Does a common theoretical framework to implement food traceability exist? Food Control, 32(2), 409-417.

Khachaturyan, M., Peterson, E., \& Wesley, F. (2017). The Russian food and agricultural import ban. Cornhusker Economics(756). Retrieved from http://digitalcommons . unl .edu/agecon_cornhusker/756

Kharpal, A. (2013, 2 October). Why Europe is crying over spilled milk. In Europe news. CNBC. Retrieved from http://www.cnbc.com/2013/10/ 02/why-europe-is-crying-over-spilled-milk.html

Kilsby, S. (2015, 17 April). Fonterra shares lose ground. Retrieved from https://agrihq.co.nz/section/all/view/fonterra -shares-lose-ground

Kopanos, G. M., Puigjaner, L., \& Georgiadis, M. C. (2009). Optimal production scheduling and lot-sizing in dairy plants: the yogurt production line. Industrial \&6 Engineering Chemistry Research, 49(2), 701-718.

Krieger, K., \& Chen, D. (2015). Post-accident stock returns of aircraft manufacturers based on potential fault. Journal of air transport management, 43, 20-28.

Kumar, S., \& Schmitz, S. (2011). Managing recalls in a consumer product supply chain-root cause analysis and measures to mitigate risks. International Journal of Production Research, 49(1), 235-253.

Kwiatkowski, D., Phillips, P. C., Schmidt, P., \& Shin, Y. (1992). Testing the null hypothesis of stationarity against the alternative of a unit root: How sure are we that economic time series have a unit root? Journal of econometrics, 54(1-3), 159-178.

LIC, \& DairyNZ. (2015). New zealand dairy statistics 2014-2015. Retrieved 1/03/18, from http://www.dairynz.co.nz/media/3136117/new -zealand-dairy-statistics-2014-15.pdf

LIC New Zealand. (2012). Herd testing. Retrieved 04/05/17, from http:// lic.co.nz/lic_Herd_Testing.cfm

Liu, H., Ferreira, S., Karali, B., et al. (2015, January 31-February 3). Hurricanes as news? A comparison of the impact of hurricanes on stock returns of energy companies. In Annual meeting, atlanta, georgia. 
MacKinlay, A. C. (1997). Event studies in economics and finance. Journal of economic literature, 35(1), 13-39.

Mason, K. (2014, March). A bigger and better dairy factory. In IPENZ Milk Collection Presentation. IPENZ Transportation Group. Retrieved from http://www.transportationgroup.nz/events/Branch-Southern.htm

McColl, B. J. (2011, November). Fonterra - Statement of evidence. Environment Canterbury Regional Council.

MFAT, \& Statistics New Zealand. (2016). Global New Zealand-international trade, investment, and travel profile: Year ended June 2016. Wellington: Ministry of Foreign Affairs and Trade, and Statistics New Zealand. (Available from http://www.stats.govt.nz)

MPI. (2014). Frequently asked questions: Proposals to amend dira and raw milk regulations. Retrieved from http://www.mpi.govt.nz/ news-resources/faqs/proposals-to-amend-dira-and-raw-milk -regulations

MPI. (2016, January). Mainland tasty cheese slices. Retrieved 27/04/17, from https://www.mpi.govt.nz/food-safety/food-safety-for -consumers/food-recalls/mainland-tasty-cheese-slices-250g/

MPI. (2017). About food recalls. Ministry for Primary Industries. Retrieved 12/09/17, from http://www.foodsafety.govt.nz/recalls-warnings/ about-recalls/

MPI. (2018). Food safety codes and standards. Retrieved 12/13/18, from https://www.mpi.govt.nz/food-safety/food-safety -codes-and-standards/

New Zealand Herald. (2015, March). Threat to contaminate baby formula. In New Zealand Herald. NZME. Publishing Limited.

Newman, M., \& Mounsey, Z. (2015, May). What milk price should I budget for in the season ahead? Retrieved 24/01/18, from https://www. dairynz.co.nz/news/latest-news/what-milk-price -should-i-budget-for-in-the-season-ahead/

NIWA. (2016, September). New Zealand Historic Weather Events Catalog. Retrieved 1/09/17, from https://hwe.niwa.co.nz

Nuphlo Pumps Ltd. (2013). Hydraulic drive pump. Retrieved 13/04/13, from http://www.nuphlo.co.nz/ nuphloco/pump-products/ milk-collection/ 
NZX. (2017a). NZX Company Research. Retrieved 6/09/17, from http:// companyresearch.nzx.com/deep_ar/

NZX. (2017b). SEPP/NZX $50 \operatorname{INDEX~(NZ50).~Retrieved~26/07/17,~from~}$ https://www.nzx.com/markets/nzsx/indices/NZ50

NZX Agri, Massey University, \& Callaghan Innovation. (2014, October). Using and understanding the farmgate milk price calculators (FGMPCs). Retrieved 31-03-15, from http://academy.agrihq.co.nz/Courses/Dairy/ Dairy-Data-Tools/Using-and-understanding-the-fmpc

One Financial Markets. (2017). UK Brent Oil. Retrieved 26/07/17, from http://www.onefinancialmarkets.com/market-library/ uk-brent-oil

Osorio, A. F., Brailsford, S. C., \& Smith, H. K. (2015). A structured review of quantitative models in the blood supply chain: a taxonomic framework for decision-making. International Journal of Production Research, 53(24), 7191-7212.

Parodi, P. W. (2001). Cow's milk components with anti-cancer potential. Australian Journal of Dairy Technology, 56(2), 65-73.

Pouliot, S., \& Sumner, D. A. (2008). Traceability, liability, and incentives for food safety and quality. American Journal of Agricultural Economics, $90(1), 15-27$.

Pozo, V. F., \& Schroeder, T. C. (2015). Costs of meat and poultry recalls to food firms. All Current Publications, Paper 687. Retrieved from http:// digitalcommons. usu.edu/extension_curall/687

Radio New Zealand. (2013, 11 September). Canterbury cleans up after fierce storm. Retrieved 15/04/17, from http://www.radionz.co.nz/ news/national/221263/canterbury-cleans-up-after-fierce-storm

Radio New Zealand. (2016, 24 March). Flooding hits west coast, nelson, tasman. Retrieved from http://www.radionz.co.nz/news/national/ 299765/flooding-hits-west-coast, -nelson,-tasman

Raghavan, N. S. (1998). Performance analysis and scheduling of manufacturing supply chain networks (Unpublished doctoral dissertation). Ph. D Thesis, Indian Institute of Science, Bangalore.

Resende-Filho, M. A., \& Buhr, B. L. (2010). Economics of traceability for mitigation of food recall costs (MPRA Paper). University Library of Munich, Germany. Retrieved from http://EconPapers.repec.org/RePEc: pra:mprapa: 3650 
Reuters. (2013, 11 August). Fonterra withdraws milk powder from sri lanka. In The international herald tribune. The New York Times. Retrieved from http://www.nytimes.com/2013/08/12/business/global/new-zealand -dairy-giant-pulls-milk-powder-from-sri-lanka.html

Rotherham, F. (2015, 25 March). Fonterra shareholders disappointed with Fonterra dividend given low milk prices. National Business Review. Retrieved from http://preview.tinyurl.com/nbr-fonterra

Rural Delivery. (2016, August). Milk fingerprinting at Fonterra. https://www.ruraldelivery.net.nz/stories/Milk-Fingerprinting -at-Fonterra. Retrieved 02/05/17, from https://www.ruraldelivery .net.nz/stories/Milk-Fingerprinting-at-Fonterra

Said, S. E., \& Dickey, D. A. (1984). Testing for unit roots in autoregressivemoving average models of unknown order. Biometrika, 71 (3), 599-607.

Salin, V., \& Hooker, N. H. (2001). Stock market reaction to food recalls. Review of Agricultural Economics, 23(1), 33-46.

Salinger, M. (1992). Standard errors in event studies. Journal of Financial and Quantitative Analysis, 27(1), 39-53.

Shitandi, A., \& Kihumbu, G. (2004). Laboratory evaluation of the improved tube test detection limits for $\beta$-lactam residues in kenyan milk. African journal of Biotechnology, 3(1), 82-87.

Skoglund, T., \& Dejmek, P. (2007). Fuzzy traceability: a process simulation derived extension of the traceability concept in continuous food processing. Food and bioproducts processing, 85(4), 354-359.

Song, J.-S., \& Zipkin, P. H. (1996). Inventory control with information about supply conditions. Management science, 42(10), 1409-1419.

Statistical Office of the European Communities. (2009). Export and import price index manual: Theory and practice. International Monetary Fund.

Statistics New Zealand. (2016, 7 September). Trade with China nearly tripled in past decade. Retrieved 26/07/17, from http://m.stats.govt.nz/browse_for_stats/industry_sectors/ imports_and_exports/trade-china-tripled-decade.aspx

Statistics New Zealand. (2017a). New zealand business demography statistics: At february 2017. Retrieved 15/13/18, from https://www.stats.govt.nz/information-releases/new-zealand -business-demography-statistics-at-february-2017 
Statistics New Zealand. (2017b). NZ.Stat. Retrieved 22/08/17, from http:// nzdotstat . stats .govt.nz/wbos/Index . aspx?

Steijns, J. M. (2001). Milk ingredients as nutraceuticals. International Journal of Dairy Technology, 54(3), 81-88.

Stojkov, K., Noy, I., \& Sağlam, Y. (2016). The trade impacts of a food scare: The Fonterra contamination incident. (Available online: http:// hdl.handle.net/10063/4969)

Tamayo, S., Monteiro, T., \& Sauer, N. (2009). Deliveries optimization by exploiting production traceability information. Engineering Applications of Artificial Intelligence, 22(4), 557-568.

Tomé, D., \& Debabbi, H. (1998). Physiological effects of milk protein components. International dairy journal, 8(5), 383-392.

Tomlin, B. (2006). On the value of mitigation and contingency strategies for managing supply chain disruption risks. Management Science, 52(5), 639-657.

Trienekens, J., \& Zuurbier, P. (2008). Quality and safety standards in the food industry, developments and challenges. International Journal of Production Economics, 113(1), 107-122. doi: 10.1016/j.ijpe.2007.02.050

Tsuda, H., Sekine, K., Ushida, Y., Kuhara, T., Takasuka, N., Iigo, M., ... Moore, M. A. (2000). Milk and dairy products in cancer prevention: focus on bovine lactoferrin. Mutation Research/Reviews in Mutation Research, 462(2), 227-233.

Wang, G., Li, Q., \& You, J. (2008). On wheat storage period estimation using markov_ chain analysis. In Industrial informatics, 2008. indin 2008. 6th ieee international conference on (pp. 882-887).

Wang, X., \& Li, D. (2006). Value added on food traceability: a supply chain management approach. In Service operations and logistics, and informatics, 2006. soli'06. ieee international conference on (pp. 493-498).

Western, M. (2011, July). Animal products (dairy processing specifications) notice 2011. PO Box 2526, Wellington.

White, H. (1980). A heteroskedasticity-consistent covariance matrix estimator and a direct test for heteroskedasticity. Econometrica, 817-838.

Workman, D. (2017, 23 June). Top milk exporting countries. Retrieved from http://www.worldstopexports.com/top-milk-exporting -countries/ 
WPC 2013 Response Team. (2013, August). Whey protein concentrate incident tracing and verification report. Retrieved 20/03/18, from https://www.mpi.govt.nz/news-and-resources/resources/ official-information-act-responses/whey-protein-concentrate -contamination-incident/ 